\title{
Palladium-Catalyzed (3+3) Annulation of Allenylethylene Carbonates with Nitrile Oxides
}

Ting Pan,${ }^{\dagger}$ Xing Gao,${ }^{\dagger}$ Sen Yang, ${ }^{\dagger}$ Lan Wang, ${ }^{\dagger}$ Yimin Hu,${ }^{\dagger}$ Min Liu,,${ }^{\dagger}$ Wei Wang,,${ }^{\ddagger}$ Yongjun $\mathrm{Wu},{ }^{\ddagger}$ Bing Zheng, ${ }^{\dagger}$ Hongchao Guo* ${ }^{\star \$}$

${ }^{\dagger}$ Department of Chemistry and Innovation Center of Pesticide Research, China Agricultural University, Beijing 100193, P. R. China

College of Public Health, Zhengzhou University, Zhengzhou 450001, P. R. China

${ }^{\S}$ Department of Nutrition and Health, China Agricultural University, Beijing 100193, P. R. China

Email: hchguo@cau.edu.cn

\section{Contents}

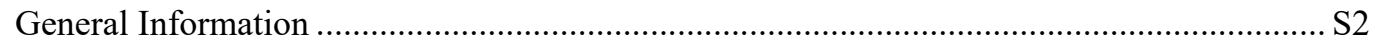

General Procedure for Preparation of Substituted AECs …………................................ S2

General Procedure for Preparation of Nitrile Oxides ............................................................ S4

General Procedure for (3+3) Annulation Reaction............................................................ S5

The Scaled-up Reaction and Further Transformations of the Products.................................. S6

Optimization of Reaction Conditions for Asymmetric (3+3) Annulation ............................ S7

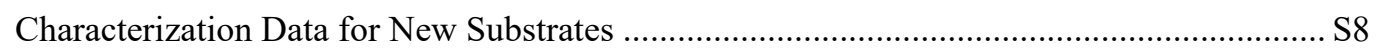

Characterization Data for All Products ............................................................................ S19

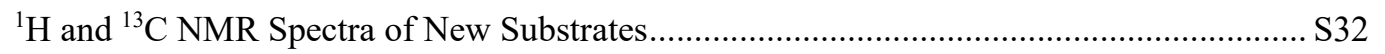

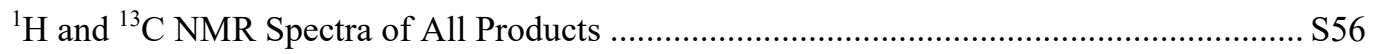

HPLC Chromatogram of Chiral Product 3aa ………….................................................... S82

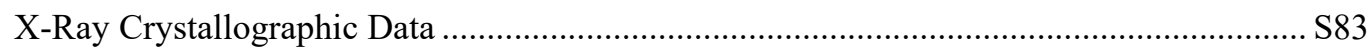




\section{General Information}

All reactions were performed under an Argon atmosphere in oven-dried glassware with magnetic stirring. Unless otherwise stated, all reagents were purchased from commercial suppliers and used without further purification. All solvents were purified and dried according to standard methods prior to use. Organic solutions were concentrated under reduced pressure using a rotary evaporator or oil pump. Reactions were monitored through thin-layer chromatography (TLC) on silica gel-precoated glass plates. Visualization on TLC was achieved by use of UV light $(254 \mathrm{~nm})$, iodine or basic $\mathrm{KMnO}_{4}$ indicator. Flash column chromatography was performed using Qingdao Haiyang flash silica gel (200-300 mesh). Infrared spectra were recorded using a Bruker Optics TENSOR 27 instrument. ${ }^{1} \mathrm{H}$ and ${ }^{13} \mathrm{C}$ NMR spectra were recorded in $\mathrm{CDCl}_{3}$ using a Bruker $300 \mathrm{MHz}, 400 \mathrm{MHz}$ and $500 \mathrm{MHz}$ of NMR instrument (referenced internally to $\mathrm{Me}_{4} \mathrm{Si}$ ). Chemical shifts $(\delta, \mathrm{ppm})$ are relative to tetramethylsilane (TMS) with the resonance of the non-deuterated solvent or TMS as the internal standard. ${ }^{1} \mathrm{H}$ NMR data are reported as follows: chemical shift, multiplicity (s = singlet; $\mathrm{d}=$ doublet; $\mathrm{t}=$ triplet; $\mathrm{q}=$ quartet; $\mathrm{p}=$ pentet; $\mathrm{dd}=$ doublet of doublets; $\mathrm{td}=$ triplet of doublets; $\mathrm{dt}=$ doublet of triplets; $\mathrm{dq}=$ doublet of quartets; $\mathrm{ddd}=$ doublet of doublet of doublets; $\mathrm{m}=$ multiplet; br = broad), coupling constant $(\mathrm{Hz})$, and integral. Data for ${ }^{13} \mathrm{C}$ NMR spectra are reported in terms of chemical shift. HRMS analyses were carried out on a Thermo Q-Exactive high resolution mass spectrometer (Thermo Scientific, Waltham, MA, USA) apparatus. The type of mass analyzer used for HRMS measurement is TOF. Data were analyzed using instrument-supplied software Xcalibur Qual Browser. X-ray crystallographic data were collected using a Bruker SMART CCD-based diffractometer equipped with a low-temperature apparatus operated at $173.15 \mathrm{~K}$. In addition, unless noted otherwise, in the reactions that need heating, the heat source is oil bath.

\section{General Procedure for Preparation of Substituted AECs}

\section{2-Hydroxyketones S2 were prepared from the 2-bromoketones S1}

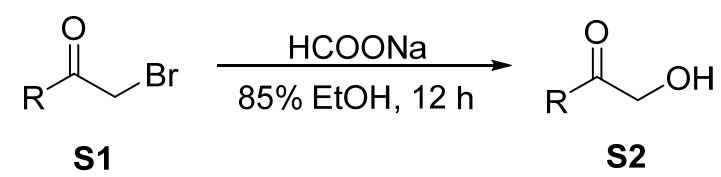

2-bromoketone $\mathbf{S 1}$ (20 mmol) and sodium formate (15 mmol, $10 \mathrm{~g}$ ) were dissolved in $40 \mathrm{~mL}$ of $85 \%$ ethanol and the resulting mixture was heated, stirred and refluxed for about 12 hours. The reaction was monitored by TLC. Once the reaction was complete, the mixture was cooled to room temperature and the ethanol was removed through rotary evaporator. $20 \mathrm{~mL}$ of water and $40 \mathrm{~mL}$ of ethyl acetate were added to the residual mixture, and the resulting mixture was then transferred to the separating funnel. The organic layer was separated and the aqueous layer was extracted with ethyl acetate $(40 \mathrm{~mL} \times 3)$ for three times. The combined organic layers were dried over anhydrous $\mathrm{Na}_{2} \mathrm{SO}_{4}$, filtered and concentrated. The residue 2-hydroxyketone $\mathbf{S 2}$ was used in next step without further purification. 


\section{AECs were prepared from the diol S3, which were prepared from ketols S2 using Method}

\section{A or Method B}

\section{Method A}

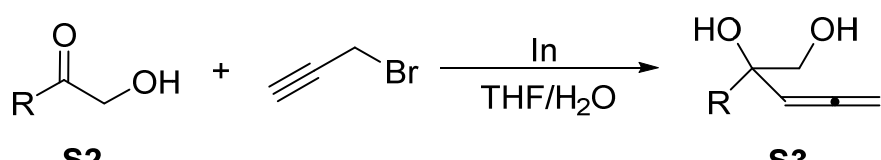

S2

S3

Propargyl bromide (1.8 equiv.) and the 2-hydroxyketone $\mathbf{S 2}$ from the previous step (1 equiv.) were dissolved in $60 \mathrm{~mL}$ of mixed solvents (THF: $\mathrm{H}_{2} \mathrm{O}=3: 1$ ). Indium powder (1.5 equiv.) was added into the above solution and the resulting mixture was stirred at $40{ }^{\circ} \mathrm{C}$ overnight. The reaction was monitored by TLC. Once the starting material was consumed, THF was removed through rotary evaporator. $30 \mathrm{~mL}$ of ethyl acetate and $20 \mathrm{~mL}$ of water were added to the residual mixture, and the resulting mixture was then transferred to the separating funnel. The organic layer was separated and the aqueous layer was extracted with ethyl acetate $(30 \mathrm{~mL} \times 3)$ for three times. The combined organic layers were dried over anhydrous $\mathrm{Na}_{2} \mathrm{SO}_{4}$, filtered and concentrated. The residue was used in next step without further purification (Note: the residue always contained propargyl diol, which could not be easily removed by flash column).

\section{Method B}

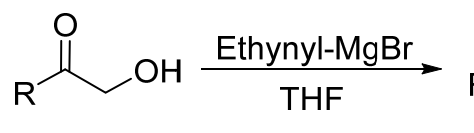

S2

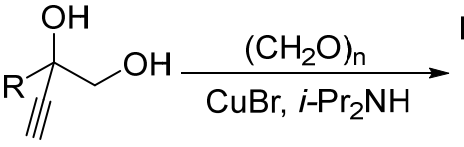

S4

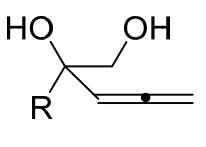

S3

To a solution of 2-hydroxyketone $\mathbf{S 2}$ from the previous step (1 equiv.) in THF (20 mL) was added ethynylmagnesium bromide $\left(0.5 \mathrm{M}\right.$ in THF, 2.5 equiv.) at $0^{\circ} \mathrm{C}$. The reaction mixture was stirred at room temperature under Ar atmosphere for $2 \mathrm{~h}$, and then the reaction was quenched with saturated aqueous $\mathrm{NH}_{4} \mathrm{Cl} .2 \mathrm{M}$ of $\mathrm{HCl}$ solution was added until the mixture became clear. The resulting mixture was transferred to the separating funnel and separated. The obtained aqueous layer was extracted with ethyl acetate $(30 \mathrm{~mL} \times 3)$ for three times. The combined organic layers were washed by brine, dried over anhydrous $\mathrm{Na}_{2} \mathrm{SO}_{4}$, filtered and concentrated to give the crude $\mathbf{S 4}$, which was used in next step without further purification. The mixture of paraformaldehyde (1.5 equiv.) and anhydrous $\mathrm{CuBr}(0.5$ equiv. $)$ in dioxane $(50 \mathrm{~mL})$ was added to the above propargylic alcohol (1 equiv.) and diisopropylamine (1.5 equiv.). The resulting mixture was heated to $90^{\circ} \mathrm{C}$ and continued to be stirred. The reaction was monitored by TLC. Once the reaction was complete, the mixture was cooled to room temperature and filtered by short silica gel column. The solvent was removed under vacuum and the residue was used in next step without further purification (Note: the residue always contained propargyl diol, which could not be easily removed by flash column). 


\section{AECs were prepared from the diol S3}

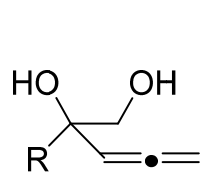

S3

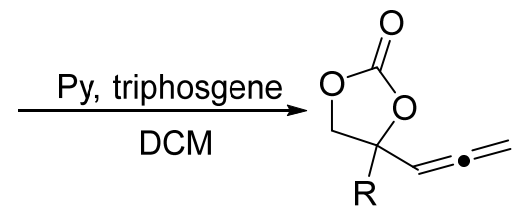

1

To a solution of diol $\mathbf{S 3}$ (1 equiv.) and pyridine (4 equiv.) in $\mathrm{CH}_{2} \mathrm{Cl}_{2}(20 \mathrm{~mL})$ was added triphosgene ( 0.5 equiv., $1.0 \mathrm{M}$ in $\mathrm{CH}_{2} \mathrm{Cl}_{2}$ ) at $0{ }^{\circ} \mathrm{C}$. The reaction mixture was stirred at room temperature under $\mathrm{Ar}$ atmosphere. Once the starting material was consumed, the reaction was quenched with $\mathrm{H}_{2} \mathrm{O}$ at once, and the mixture was extracted with $\mathrm{CH}_{2} \mathrm{Cl}_{2}(20 \mathrm{~mL} \times 3)$ for three times. The combined organic layers were dried over anhydrous $\mathrm{Na}_{2} \mathrm{SO}_{4}$, filtered and concentrated. The residue was purified by flash column to afford the corresponding AECs $\mathbf{1}$ (petroleum ether/ ethyl acetate as the eluent) (Note: propargyl cyclic carbonate was always formed from propargyl diol and isomerization of allenyl cyclic carbonate, but could be removed through flash column).

\section{General Procedure for Preparation of Nitrile Oxides}

Nitrile Oxides 2a were prepared from the oxime S6 which were prepared from aromatic aldehyde 55

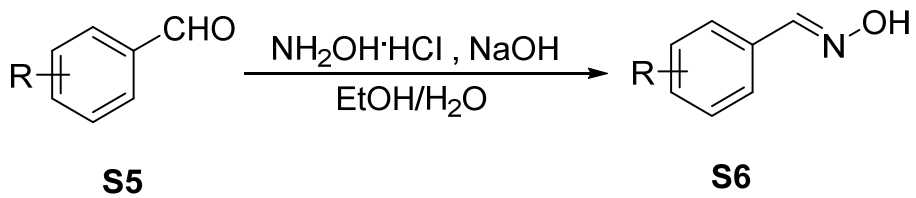

A solution of aromatic aldehydes $\mathbf{S} 5$ (1.0 equiv.) in $80 \mathrm{~mL}$ of ethanol was stirred at room temperature, and hydroxylamine hydrochloride (1.1 equiv.) in $40 \mathrm{~mL}$ of $\mathrm{H}_{2} \mathrm{O}$ was added the reaction system and the $\mathrm{NaOH}$ aqueous solution (1.2 equiv.) was added dropwise into the reaction system at $0{ }^{\circ} \mathrm{C}$. The reaction mixture was stirred overnight at room temperature. After the reaction, the obtained aqueous layer was extracted with dichloromethane, combined with organic layer, washed once with saturated salt solution, and then the combined organic layers were dried over anhydrous $\mathrm{Na}_{2} \mathrm{SO}_{4}$, filtered and concentrated to afford the corresponding oxime S6.

\section{The nitrile oxides 2 a were prepared from the oxime S6}

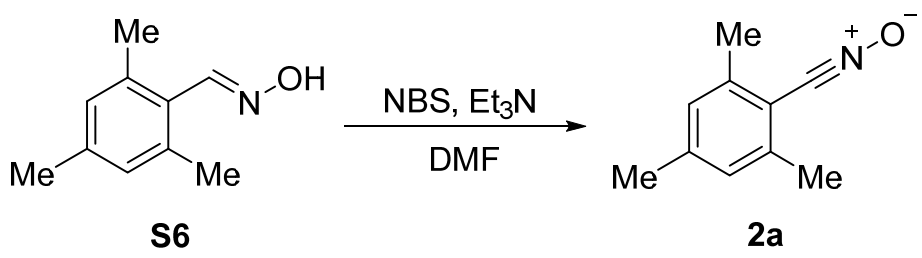

The oxime $\mathbf{S 6}$ was dissolved in $40 \mathrm{~mL}$ of DMF and the N-bromosuccinimide (NBS) (1.0 equiv.) was dissolved in $40 \mathrm{~mL}$ of DMF and added dropwise into the reaction system at $0{ }^{\circ} \mathrm{C}$. Then the triethylamine (1.5 equiv.) dissolve in $20 \mathrm{~mL}$ of DMF and added it into the reaction system drop 
by drop. The reaction mixture was stirred at room temperature. After the reaction is complete (monitored by TLC), ice water is added dropwise to the system until no white precipitate is left at $0{ }^{\circ} \mathrm{C}$. The reaction continued to stand still at $0{ }^{\circ} \mathrm{C}$ for 4 hours, and filter. The obtained filter residue is dried and recrystallized with methanol, and the target product $\mathbf{2 a}$ was afforded.

\section{The nitrile oxides $2 \mathrm{~b}-\mathrm{d}$ were prepared from the oxime $\mathrm{S6}$}

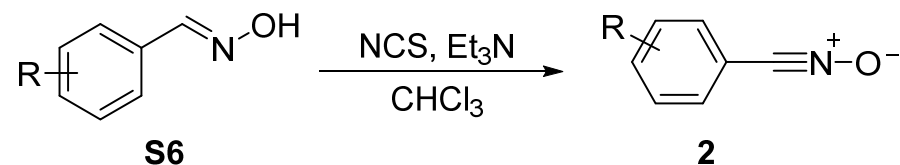

The oxime $\mathbf{S 6}$ was dissolved in $40 \mathrm{~mL}$ of $\mathrm{CHCl}_{3}$ and the $\mathrm{N}$-chlorosuccinimide (NCS) (1.0 equiv.) was dissolved in $40 \mathrm{~mL}$ of $\mathrm{CHCl}_{3}$ and added dropwise into the reaction system at $0{ }^{\circ} \mathrm{C}$. Then the triethylamine (1.5 equiv.) dissolve in $20 \mathrm{~mL}_{\text {of }} \mathrm{CHCl}_{3}$ and added it into the reaction system drop by drop. The reaction mixture was stirred at room temperature. After the reaction is complete (monitored by TLC), the reaction mixture was transferred to the separating funnel and washed with $\mathrm{H}_{2} \mathrm{O}(20 \mathrm{~mL} \times 3)$. The combined organic layers were dried over anhydrous $\mathrm{Na}_{2} \mathrm{SO}_{4}$, filtered and concentrated. The residue was purified by flash chromatography on silica to afford corresponding nitrile oxides $\mathbf{2 b - \mathbf { d }}$ (petroleum ether/ ethyl acetate as the eluent).

General Procedure for (3+3) Annulation Reaction of AECs 1 with Nitrile Oxides 2 For Table 2<smiles>[R]c1ccccc1C1(C=C=C)COC(=O)O1</smiles>

1<smiles>Cc1cc(C)c(C#[N+][O-])c(C)c1</smiles>

2a

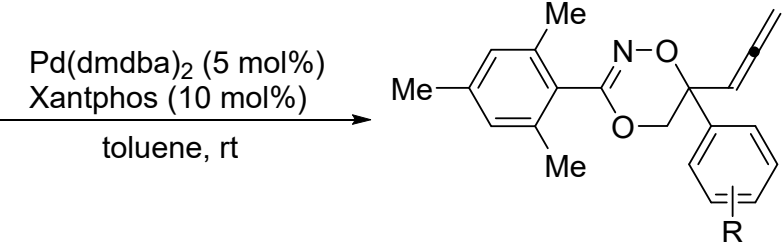

An oven-dried $10 \mathrm{~mL}$ of Schlenk tube was charged with AECs $1(0.15 \mathrm{mmol})$, nitrile oxide 2a $(0.1 \mathrm{mmol}), \mathrm{Pd}(\mathrm{dmdba})_{2}(0.05$ equiv, $4 \mathrm{mg})$, Xantphos $(0.10$ equiv, $3 \mathrm{mg})$ in $1 \mathrm{~mL}$ of toluene under $\mathrm{Ar}$ atmosphere at $25^{\circ} \mathrm{C}$. Once the starting material was completely consumed (monitored by TLC), the mixture was concentrated to dryness. The residue was purified by flash column chromatography to afford the product $\mathbf{3}$ (petroleum ether/EtOAc as the eluent).

\section{For Table 3}

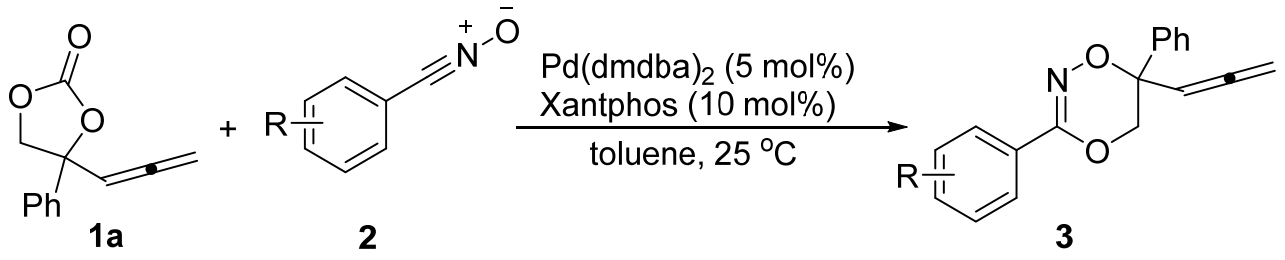

An oven-dried $10 \mathrm{~mL}$ of Schlenk tube was charged with AEC 1a $(0.15 \mathrm{mmol})$, nitrile oxide 2 ( $0.1 \mathrm{mmol})$, Pd (dmdba) 2 ( 0.05 equiv, $4 \mathrm{mg}$ ), Xantphos ( 0.10 equiv, $3 \mathrm{mg}$ ) in $1 \mathrm{~mL}$ of toluene under $\mathrm{Ar}$ atmosphere at $25^{\circ} \mathrm{C}$. Once the starting material was completely consumed (monitored 
by TLC), the mixture was concentrated to dryness. The residue was purified by flash column chromatography to afford the product $\mathbf{3}$ (petroleum ether/EtOAc as the eluent).

\section{The Scaled-up Reaction}

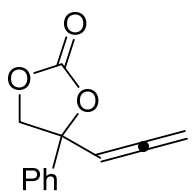

$1 \mathrm{a}$

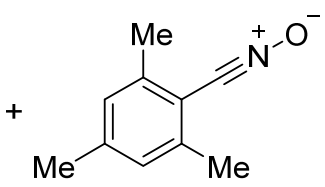

2a

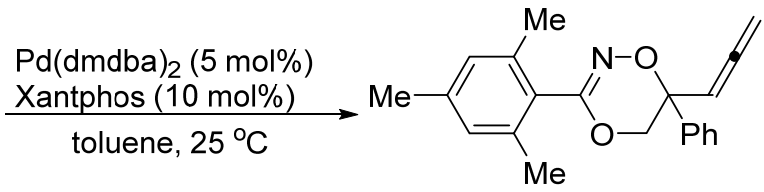

3aa, $253 \mathrm{mg}, 79 \%$ yield

$(1.5 \mathrm{mmol}, 303 \mathrm{mg}) \quad(1 \mathrm{mmol}, 161 \mathrm{mg})$

An oven-dried $100 \mathrm{~mL}$ of Schlenk tube was charged with AEC 1a (303 mg $1.5 \mathrm{mmol})$, nitrile oxide 2a (161 mg, $1.0 \mathrm{mmol}), \mathrm{Pd}$ (dmdba) $)_{2}(0.05$ equiv, $4 \mathrm{mg}$ ), Xantphos ( 0.10 equiv, $3 \mathrm{mg}$ ) in $15 \mathrm{~mL}$ of toluene under Ar atmosphere at $25{ }^{\circ} \mathrm{C}$. Once the starting material was completely consumed (monitored by TLC), the mixture was concentrated to dryness. The residue was purified by flash column chromatography (petroleum ether/EtOAc as the eluent) to afford the product 3aa ( $253 \mathrm{mg}, 79 \%$ yield).

\section{Further Transformations of the Products}

\section{Synthesis of compound 4}

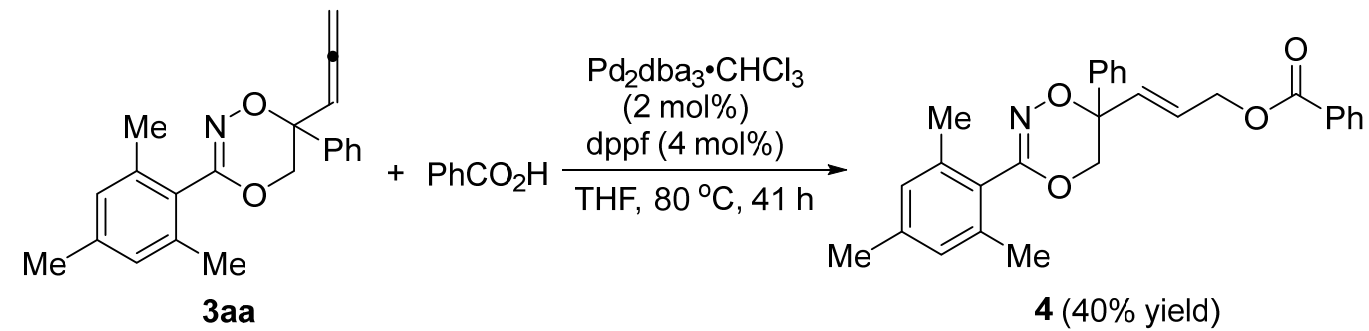

An oven-dried $10 \mathrm{~mL}$ of Schlenk tube was charged with the product 3aa (16 mg, $0.05 \mathrm{mmol})$, benzoic acid (7.3 mg, $0.06 \mathrm{mmol}), \mathrm{Pd}_{2}\left(\mathrm{dba}_{3} \cdot \mathrm{CHCl}_{3}(2.0 \mathrm{mmol} \%)\right.$, dppf $(4.0 \mathrm{mmol} \%)$ in $15 \mathrm{~mL}$ of THF under $\mathrm{Ar}$ atmosphere at $80{ }^{\circ} \mathrm{C}$. Once the starting material was completely consumed (monitored by TLC), the mixture was concentrated to dryness. The residue was purified by flash column chromatography (petroleum ether/EtOAc as the eluent) to afford the product 4 (8.6 mg, $40 \%$ yield).

\section{Synthesis of compound 5}

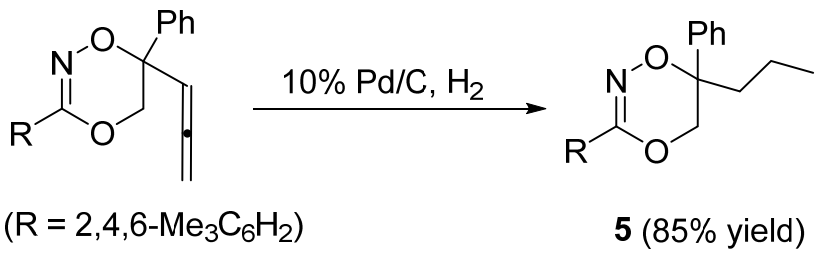

To a dry tube equipped with a stirring bar added 3aa $(16 \mathrm{mg}, 0.05 \mathrm{mmol}), \mathrm{Pd} / \mathrm{C}(1.06 \mathrm{mg}$, $0.01 \mathrm{mmol})$ and anhydrous $\mathrm{MeOH}(1 \mathrm{~mL})$. The reaction mixture was stirred under an atmosphere of hydrogen at $\mathrm{rt}$ for overnight. Upon completion of the reaction (monitored by TLC), the mixture was concentrated to dryness. The residue was purified by flash column (petroleum ether/EtOAc as the eluent) to afford the product 5 (13.8 $\mathrm{mg}, 85 \%$ yield). 
Table S1. Optimization of Reaction Conditions for Asymmetric (3+3) Cycloaddition of AEC 1a with Nitrile Oxide $\mathbf{2 a}^{a}$

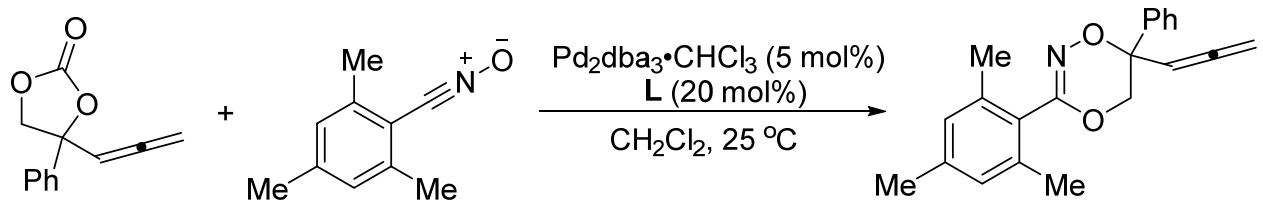

$1 \mathrm{a}$

$2 a$

3aa<smiles></smiles>

L1<smiles>Pc1cccc2c1O[C@H]1CCC[C@@H](C2)[C@@]12Cc1cccc(P)c1O2</smiles>

L2

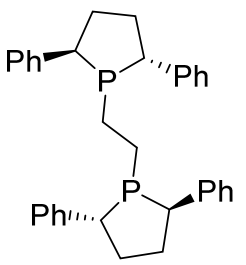

L3

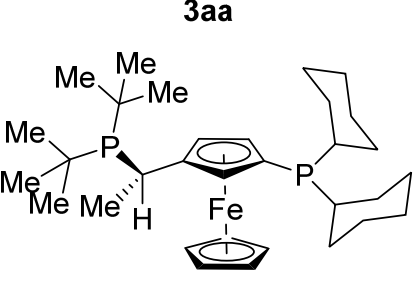

L4<smiles>FC(F)(F)Oc1ccc(-c2ccccc2)c(-c2c(-c3ccccc3)ccc3c2OC(F)(F)O3)c1</smiles>

L5

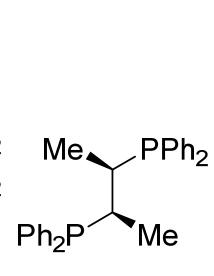

L6<smiles>CC(C)[C@H]1COC(c2ccccc2PP)=N1</smiles>

L7

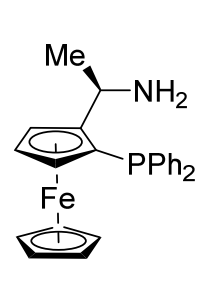

L8

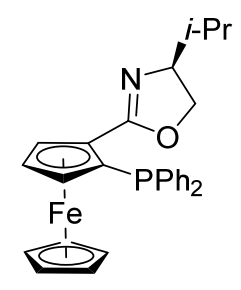

L9

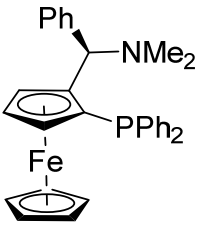

L10

\begin{tabular}{ccccc} 
entry & ligand & $t / h$ & 3aa yield $(\%)^{b}$ & ee $(\%)^{c}$ \\
1 & $\mathbf{L 1}$ & 72 & trace & - \\
2 & $\mathbf{L 2}$ & 72 & 11 & 12 \\
3 & $\mathbf{L 3}$ & 72 & trace & - \\
5 & $\mathbf{L 4}$ & 72 & 6 & 7 \\
6 & $\mathbf{L 5}$ & 48 & trace & - \\
7 & $\mathbf{L 6}$ & 72 & trace & - \\
8 & $\mathbf{L 7}$ & 48 & trace & - \\
9 & $\mathbf{L 8}$ & 72 & 17 & 29 \\
10 & $\mathbf{L 9}$ & 72 & 16 & 53 \\
11 & $\mathbf{L 1 0}$ & 72 & trace & - \\
\hline
\end{tabular}

${ }^{a}$ Unless noted otherwise, the reaction of $\mathbf{1 a}(0.15 \mathrm{mmol}), \mathbf{2 a}(0.10 \mathrm{mmol}), \mathrm{Pd}_{2} \mathrm{dba}_{3} \cdot \mathrm{CHCl}_{3}(5$ mol\%) and ligand (20 mol\% for phosphoramidite, $10 \mathrm{~mol} \%$ for diphosphines) was performed in $1.0 \mathrm{~mL}$ of $\mathrm{CH}_{2} \mathrm{Cl}_{2}$ under indicated reaction conditions. ${ }^{b}$ Isolated yield. ${ }^{c}$ Determined by chiral HPLC analysis. 


\section{Characterization Data for New Substrates}

\section{4-phenyl-4-(propa-1,2-dien-1-yl)-1,3-dioxolan-2-one (1a)}<smiles>C=CCC1(c2ccccc2)COC(=O)O1</smiles>

Prepared according to the general procedure (method A) as described above from the starting material 2-hydroxyacetophenone a (4 g, $30 \mathrm{mmol})$. The overall yield calculated based on amount of 2-hydroxyacetophenone a: $11 \%$ yield $(654 \mathrm{mg})$. It was isolated by simple filtration and dried to afford a yellow liquid. ${ }^{1} \mathrm{H}$ NMR $\left(500 \mathrm{MHz}, \mathrm{CDCl}_{3}\right) \delta 7.5-7.4(\mathrm{~m}, 5 \mathrm{H}), 5.6(\mathrm{t}, J=$ $6.6 \mathrm{~Hz}, 1 \mathrm{H}), 5.2-5.1(\mathrm{~m}, 2 \mathrm{H}), 4.8(\mathrm{~d}, J=8.5 \mathrm{~Hz}, 1 \mathrm{H}), 4.4(\mathrm{dd}, J=8.5,0.8 \mathrm{~Hz}, 1 \mathrm{H}) ;{ }^{13} \mathrm{C} \mathrm{NMR}$ $\left(126 \mathrm{MHz}, \mathrm{CDCl}_{3}\right) \delta 207.3,154.1,139.4,129.0,129.0,124.6,93.4,84.2,81.1,74.0$; IR (thin film) $v_{\max } 3068,1956,1801,1475,1450,1378,1333,1211,1153,1086,1064,1001,860,765$, 729, 700, $552 \mathrm{~cm}^{-1}$; HRMS (ESI) calculated for $\mathrm{C}_{12} \mathrm{H}_{10} \mathrm{NaO}_{3}[\mathrm{M}+\mathrm{n} \mathrm{Na}]^{+} 225.0522$, found 225.0506 .

\section{4-(2-fluorophenyl)-4-(propa-1,2-dien-1-yl)-1,3-dioxolan-2-one (1b)}<smiles>C=CC1(c2ccccc2F)COC(=O)O1</smiles>

Prepared according to the general procedure (method B) as described above from the starting material 2-bromoketone $\mathbf{b}(4.3 \mathrm{~g}, 20 \mathrm{mmol})$. The overall yield calculated based on amount of 2-bromoketone $\mathbf{b}$ : $6 \%$ yield $(250 \mathrm{mg})$. It was isolated by simple filtration and dried to afford a yellow solid. ${ }^{1} \mathrm{H}$ NMR (400 MHz, $\left.\mathrm{CDCl}_{3}\right) \delta 7.63-7.54(\mathrm{~m}, 1 \mathrm{H}), 7.44-7.34(\mathrm{~m}, 1 \mathrm{H}), 7.28-$ $7.19(\mathrm{~m}, 1 \mathrm{H}), 7.16-7.06(\mathrm{~m}, 1 \mathrm{H}), 5.54(\mathrm{t}, J=6.5 \mathrm{~Hz}, 1 \mathrm{H}), 5.17-5.06(\mathrm{~m}, 2 \mathrm{H}), 4.94(\mathrm{dd}, J=$ 8.9, $2.6 \mathrm{~Hz}, 1 \mathrm{H}), 4.56(\mathrm{dd}, J=8.9,1.6 \mathrm{~Hz}, 1 \mathrm{H}) ;{ }^{13} \mathrm{C} \mathrm{NMR}\left(101 \mathrm{MHz}, \mathrm{CDCl}_{3}\right) \delta 207.0,159.8$, 157.3, 153.3, 130.7, 130.6, 126.9, 126.8, 126.1, 126.1, 124.6, 124.6, 116.0, 115.8, 92.9, 92.8, 81.9, 81.9, 81.0, 73.7, 73.6; IR (thin film) $v_{\max } 2993,1956,1809,1617,1586,1489,1453,1381$, 1331, 1214, 1154, 1110, 1072, 1004, 858, 826, 762, 640, 522, $470 \mathrm{~cm}^{-1}$; HRMS (ESI) calculated for $\mathrm{C}_{12} \mathrm{H}_{10} \mathrm{FO}_{3}[\mathrm{M}+\mathrm{H}]^{+}$221.0609, found 221.0607 . 
<smiles>C=CCC1(c2cccc(F)c2)COC(=O)O1</smiles>

Prepared according to the general procedure (method B) as described above from the starting material 2-bromoketone c $(4.3 \mathrm{~g}, 20 \mathrm{mmol})$. The overall yield calculated based on amount of 2-bromoketone c: $3 \%$ yield (134 mg). It was isolated by simple filtration and dried to afford a yellow liquid. ${ }^{1} \mathrm{H}$ NMR (400 MHz, $\left.\mathrm{CDCl}_{3}\right) \delta 7.46-7.37(\mathrm{~m}, 1 \mathrm{H}), 7.21-7.13(\mathrm{~m}, 1 \mathrm{H}), 7.16-$ $7.04(\mathrm{~m}, 2 \mathrm{H}), 5.57(\mathrm{t}, J=6.6 \mathrm{~Hz}, 1 \mathrm{H}), 5.27-5.14(\mathrm{~m}, 2 \mathrm{H}), 4.85(\mathrm{~d}, J=8.5 \mathrm{~Hz}, 1 \mathrm{H}), 4.37(\mathrm{dd}$, $J=8.5,0.7 \mathrm{~Hz}, 1 \mathrm{H}) ;{ }^{13} \mathrm{C} \mathrm{NMR}\left(101 \mathrm{MHz}, \mathrm{CDCl}_{3}\right) \delta 207.3,164.0,161.6,153.5,141.9,141.8$, 130.7, 130.6, 120.2, 120.1, 116.0, 115.7, 112.2, 111.9, 93.0, 83.5, 83.5, 81.2, 73.7; IR (thin film) $v_{\max } 3075,1956,1808,1616,1593,1490,1446,1378,1331,1267,1212,1154,1064,943,864$, 790, 766, 731, 696, 523, $457 \mathrm{~cm}^{-1}$; HRMS (ESI) calculated for $\mathrm{C}_{12} \mathrm{H}_{9} \mathrm{FNaO}_{3}[\mathrm{M}+\mathrm{Na}]^{+} 243.0428$, found 243.0419 .

\section{4-(4-fluorophenyl)-4-(propa-1,2-dien-1-yl)-1,3-dioxolan-2-one (1d)}<smiles>O=C(CBr)c1ccc(F)cc1</smiles>

Prepared according to the general procedure (method A) as described above from the starting material 2-bromoketone $\mathbf{d}(4.3 \mathrm{~g}, 20 \mathrm{mmol})$. The overall yield calculated based on amount of 2-bromoketone $\mathbf{d}$ : $22 \%$ yield (1.46 g). It was isolated by simple filtration and dried to afford a yellow liquid. ${ }^{1} \mathrm{H}$ NMR (400 MHz, $\left.\mathrm{CDCl}_{3}\right) \delta 7.42-7.34(\mathrm{~m}, 2 \mathrm{H}), 7.17-7.06(\mathrm{~m}, 2 \mathrm{H}), 5.56(\mathrm{t}$, $J=6.6 \mathrm{~Hz}, 1 \mathrm{H}), 5.24-5.11(\mathrm{~m}, 2 \mathrm{H}), 4.83(\mathrm{~d}, J=8.6 \mathrm{~Hz}, 1 \mathrm{H}), 4.36(\mathrm{dd}, J=8.6,0.8 \mathrm{~Hz}, 1 \mathrm{H})$; ${ }^{13} \mathrm{C}$ NMR $\left(101 \mathrm{MHz}, \mathrm{CDCl}_{3}\right) \delta 207.3,164.0,161.5,153.7,135.1,135.1,126.7,126.6,116.0$, 115.7, 93.2, 83.8, 81.1, 73.9; IR (thin film) $v_{\max } 3074,1955,1801,1704,1600,1510,1476$, 1412, 1377, 1331, 1266, 1225, 1154, 1104, 1062, 992, 860, 835, 787, 767, 732, 702, 649, 631, $603,578,544,509,437 \mathrm{~cm}^{-1}$; HRMS (ESI) calculated for $\mathrm{C}_{12} \mathrm{H}_{10} \mathrm{FO}_{3}[\mathrm{M}+\mathrm{H}]^{+} 221.0609$, found 221.0609 . 


\section{4-(3-chlorophenyl)-4-(propa-1,2-dien-1-yl)-1,3-dioxolan-2-one (1e)}<smiles>C=CC1(c2cccc(Cl)c2)COC(=O)O1</smiles>

Prepared according to the general procedure (method A) as described above from the starting material 2-bromoketone e ( $4.7 \mathrm{~g}, 20 \mathrm{mmol})$. The overall yield calculated based on amount of 2-bromoketone e: $9 \%$ yield $(390 \mathrm{mg})$. It was isolated by simple filtration and dried to afford a yellow liquid. ${ }^{1} \mathrm{H}$ NMR $\left(500 \mathrm{MHz}, \mathrm{CDCl}_{3}\right) \delta 7.5-7.4(\mathrm{~m}, 2 \mathrm{H}), 7.3-7.2(\mathrm{~m}, 2 \mathrm{H}), 5.5(\mathrm{t}, J=$ $6.6 \mathrm{~Hz}, 1 \mathrm{H}), 5.2-5.1(\mathrm{~m}, 2 \mathrm{H}), 4.8(\mathrm{~d}, J=8.6 \mathrm{~Hz}, 1 \mathrm{H}), 4.3(\mathrm{~d}, J=8.6 \mathrm{~Hz}, 1 \mathrm{H}),{ }^{13} \mathrm{C}$ NMR $(126$ $\left.\mathrm{MHz}, \mathrm{CDCl}_{3}\right) \delta 206.4,152.6,140.6,131.1,129.6,126.9,122.3,122.1,92.0,82.5,80.5,72.7$; IR (thin film) $v_{\max } 3069,1954,1800,1594,1569,1475,1420,1377,1327,1209,1153,1100$, 1063, 996, 859, 787, 768, 747, 715, 693, $437 \mathrm{~cm}^{-1}$; HRMS (ESI) calculated for $\mathrm{C}_{12} \mathrm{H}_{8} \mathrm{ClO}_{3}$ [M$\mathrm{H}^{-} 235.0167$, found 235.0160 .

\section{4-(4-chlorophenyl)-4-(propa-1,2-dien-1-yl)-1,3-dioxolan-2-one (1f)}<smiles>C=CC1(c2ccc(Cl)cc2)COC(=O)O1</smiles>

Prepared according to the general procedure (method A) as described above from the starting material 2-bromoketone $\mathbf{f}(4.7 \mathrm{~g}, 20 \mathrm{mmol})$. The overall yield calculated based on amount of 2 bromoketone f: $13 \%$ yield $(580 \mathrm{mg})$. It was isolated by simple filtration and dried to afford a yellow solid. ${ }^{1} \mathrm{H}$ NMR $\left(500 \mathrm{MHz}, \mathrm{CDCl}_{3}\right) \delta 7.3(\mathrm{~d}, J=8.5 \mathrm{~Hz}, 2 \mathrm{H}), 7.3(\mathrm{~d}, J=8.6 \mathrm{~Hz}, 2 \mathrm{H}), 5.5$ $(\mathrm{t}, J=6.6 \mathrm{~Hz}, 1 \mathrm{H}), 5.2-5.1(\mathrm{~m}, 2 \mathrm{H}), 4.8(\mathrm{~d}, J=8.5 \mathrm{~Hz}, 1 \mathrm{H}), 4.3(\mathrm{~d}, J=8.5 \mathrm{~Hz}, 1 \mathrm{H}) ;{ }^{13} \mathrm{C} \mathrm{NMR}$ $\left(126 \mathrm{MHz}, \mathrm{CDCl}_{3}\right) \delta 206.4,152.7,136.9,134.1,128.2,125.2,92.1,82.8,80.3,72.9 ;$ IR (thin film) $v_{\max } 3297,2928,1691,1593,1491,1400,1263,1090,1036,1012,977,826,753,721$, 638, 525, $492 \mathrm{~cm}^{-1}$; HRMS (ESI) calculated for $\mathrm{C}_{12} \mathrm{H}_{10} \mathrm{ClO}_{3}[\mathrm{M}+\mathrm{H}]^{+} 237.0313$, found 237.0315. 
<smiles>C=CCC1(c2cccc(Br)c2)COC(=O)O1</smiles>

Prepared according to the general procedure (method B) as described above from the starting material 2-bromoketone $\mathbf{g}(5.5 \mathrm{~g}, 20 \mathrm{mmol})$. The overall yield calculated based on amount of 2-bromoketone $\mathrm{g}$ : $4 \%$ yield (200 $\mathrm{mg})$. It was isolated by simple filtration and dried to afford a yellow liquid. ${ }^{1} \mathrm{H}$ NMR (500 MHz, $\left.\mathrm{CDCl}_{3}\right) \delta 7.34-7.25(\mathrm{~m}, 3 \mathrm{H}), 7.21-7.18(\mathrm{~m}, 1 \mathrm{H}), 5.48(\mathrm{t}$, $J=6.6 \mathrm{~Hz}, 1 \mathrm{H}), 5.18-5.08(\mathrm{~m}, 2 \mathrm{H}), 4.76(\mathrm{~d}, J=8.5 \mathrm{~Hz}, 1 \mathrm{H}), 4.29(\mathrm{~d}, J=8.6 \mathrm{~Hz}, 1 \mathrm{H}) ;{ }^{13} \mathrm{C}$ NMR (126 MHz, $\left.\mathrm{CDCl}_{3}\right) \delta 206.4,152.6,140.4,134.1,129.4,128.2,124.1,121.8,92.1,82.6$, 80.4, 72.8; IR (thin film) $v_{\max } 3071,1955,1806,1598,1576,1476,1417,1378,1329,1251$, 1210, 1153, 1102, 1065, 1016, 860, 791, 769, 749, 728, 694, $439 \mathrm{~cm}^{-1}$; HRMS (ESI) calculated for $\mathrm{C}_{12} \mathrm{H}_{8} \mathrm{BrO}_{3}[\mathrm{M}-\mathrm{H}]^{-}$278.9662, found 278.9653.

\section{4-(4-bromophenyl)-4-(propa-1,2-dien-1-yl)-1,3-dioxolan-2-one (1h)}<smiles>C=C=CC1(c2cc(Br)ccc2C=CC=C)COC(=O)O1</smiles>

Prepared according to the general procedure (method A) as described above from the starting material 2-bromoketone $\mathbf{h}(5.5 \mathrm{~g}, 20 \mathrm{mmol})$. The overall yield calculated based on amount of 2-bromoketone $\mathbf{h}: 14 \%$ yield $(780 \mathrm{mg}$ ). It was isolated by simple filtration and dried to afford a yellow solid. ${ }^{1} \mathrm{H}$ NMR $\left(500 \mathrm{MHz}, \mathrm{CDCl}_{3}\right) \delta 7.5(\mathrm{~d}, J=8.6 \mathrm{~Hz}, 2 \mathrm{H}), 7.2(\mathrm{~d}, J=8.5 \mathrm{~Hz}, 2 \mathrm{H})$, $5.5(\mathrm{t}, J=6.6 \mathrm{~Hz}, 1 \mathrm{H}), 5.2-5.1(\mathrm{~m}, 2 \mathrm{H}), 4.8(\mathrm{~d}, J=8.6 \mathrm{~Hz}, 1 \mathrm{H}), 4.3(\mathrm{~d}, J=8.5 \mathrm{~Hz}, 1 \mathrm{H}) ;{ }^{13} \mathrm{C}$ NMR (126 MHz, $\left.\mathrm{CDCl}_{3}\right) \delta 206.4,152.7,137.4,131.1,125.5,122.2,92.1,82.8,80.4,72.8 ; \mathrm{IR}$ (thin film) $v_{\max } 3294,2927,1955,1691,1588,1488,1395,1261,1074,1046,1009,986,821$, 746, 718, $638 \mathrm{~cm}^{-1}$; HRMS (ESI) calculated for $\mathrm{C}_{12} \mathrm{H}_{8} \mathrm{BrO}_{3}[\mathrm{M}-\mathrm{H}]^{-} 278.9662$, found 278.9652. 


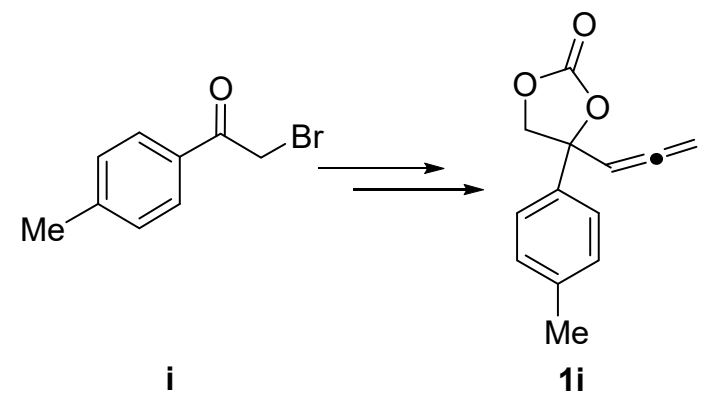

Prepared according to the general procedure (method A) as described above from the starting material 2-bromoketone i (4.2 g, $20 \mathrm{mmol})$. The overall yield calculated based on amount of 2bromoketone i: $9 \%$ yield $(390 \mathrm{mg})$. It was isolated by simple filtration and dried to afford a yellow solid. ${ }^{1} \mathrm{H}$ NMR (500 MHz, $\left.\mathrm{CDCl}_{3}\right) \delta 7.20(\mathrm{~d}, J=8.4 \mathrm{~Hz}, 2 \mathrm{H}), 7.15(\mathrm{~d}, J=8.3 \mathrm{~Hz}, 2 \mathrm{H})$, $5.49(\mathrm{t}, J=6.6 \mathrm{~Hz}, 1 \mathrm{H}), 5.14-5.03(\mathrm{~m}, 2 \mathrm{H}), 4.75(\mathrm{~d}, J=8.4 \mathrm{~Hz}, 1 \mathrm{H}), 4.29(\mathrm{~d}, J=8.6 \mathrm{~Hz}, 1 \mathrm{H})$, $2.30(\mathrm{~s}, 3 \mathrm{H}) ;{ }^{13} \mathrm{C} \mathrm{NMR}\left(126 \mathrm{MHz}, \mathrm{CDCl}_{3}\right) \delta 206.2,153.1,137.9,135.4,128.6,123.6,92.4$, 83.3, 80.0, 73.1, 20.1; IR (thin film) $v_{\max } 2921,1956,1806,1514,1475,1377,1330,1275,1260$, $1210,1186,1153,1117,1066,860,818,767,750,732,503 \mathrm{~cm}^{-1}$; HRMS (ESI) calculated for $\mathrm{C}_{13} \mathrm{H}_{11} \mathrm{O}_{3}[\mathrm{M}-\mathrm{H}]^{-}$215.0714, found 215.0705.

\section{4-(2-methoxyphenyl)-4-(propa-1,2-dien-1-yl)-1,3-dioxolan-2-one (1j)}

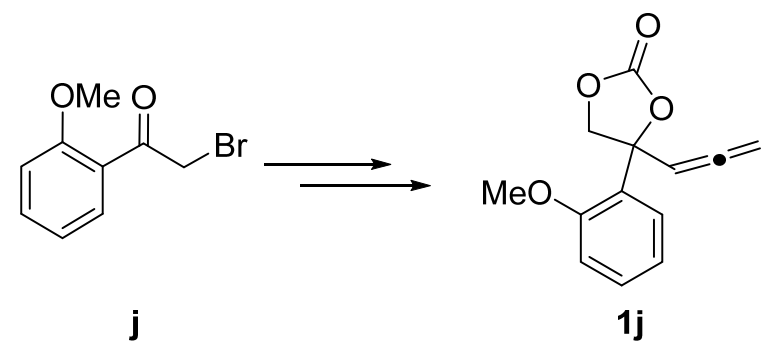

Prepared according to the general procedure (method B) as described above from the starting material 2-bromoketone j (4.6 g, $20 \mathrm{mmol}$ ). The overall yield calculated based on amount of 2bromoketone $\mathbf{j}$ : $8 \%$ yield $(360 \mathrm{mg})$. It was isolated by simple filtration and dried to afford a yellow solid. ${ }^{1} \mathrm{H}$ NMR (500 MHz, $\left.\mathrm{CDCl}_{3}\right) \delta 7.52$ (dd, $\left.J=7.7,1.7 \mathrm{~Hz}, 1 \mathrm{H}\right), 7.35$ (ddd, $J=8.3$, 7.5, 1.7 Hz, 1H), $7.01(\mathrm{td}, J=7.6,1.1 \mathrm{~Hz}, 1 \mathrm{H}), 6.92(\mathrm{dd}, J=8.2,1.0 \mathrm{~Hz}, 1 \mathrm{H}), 5.52(\mathrm{t}, J=6.6$ $\mathrm{Hz}, 1 \mathrm{H}), 5.05(\mathrm{dd}, J=6.6,1.5 \mathrm{~Hz}, 2 \mathrm{H}), 4.90(\mathrm{~d}, J=8.9 \mathrm{~Hz}, 1 \mathrm{H}), 4.47(\mathrm{~d}, J=9.0 \mathrm{~Hz}, 1 \mathrm{H}), 3.83$ (s, 3H); ${ }^{13} \mathrm{C}$ NMR $\left(126 \mathrm{MHz}, \mathrm{CDCl}_{3}\right) \delta 206.8,155.0,154.1,130.1,127.8,125.5,121.0,111.1$, 93.5, 83.0, 80.5, 74.3, 55.5; IR (thin film) $v_{\max } 2940,1957,1800,1601,1491,1465,1437,1376$, 1292, 1247, 1187, 1123, 1074, 1051, 1022, 858, 755, 729, $642 \mathrm{~cm}^{-1}$; HRMS (ESI) calculated for $\mathrm{C}_{13} \mathrm{H}_{12} \mathrm{NaO}_{4}[\mathrm{M}+\mathrm{Na}]^{+}$255.0628, found 255.0609. 
<smiles>C=CC1(c2cccc(OC)c2)COC(=O)O1</smiles>

Prepared according to the general procedure (method B) as described above from the starting material 2-bromoketone $\mathbf{k}(4.6 \mathrm{~g}, 20 \mathrm{mmol})$. The overall yield calculated based on amount of 2-bromoketone $\mathbf{k}$ : $11 \%$ yield $(520 \mathrm{mg})$. It was isolated by simple filtration and dried to afford a yellow solid. ${ }^{1} \mathrm{H}$ NMR $\left(500 \mathrm{MHz}, \mathrm{CDCl}_{3}\right) \delta 7.4-7.3(\mathrm{~m}, 1 \mathrm{H}), 7.0-6.9(\mathrm{~m}, 3 \mathrm{H}), 5.6(\mathrm{t}, J=$ $6.6 \mathrm{~Hz}, 1 \mathrm{H}), 5.2-5.1(\mathrm{~m}, 2 \mathrm{H}), 4.8(\mathrm{~d}, J=8.5 \mathrm{~Hz}, 1 \mathrm{H}), 4.4(\mathrm{~d}, J=8.5 \mathrm{~Hz}, 1 \mathrm{H}), 3.8(\mathrm{~s}, 3 \mathrm{H}) ;{ }^{13} \mathrm{C}$ NMR (126 MHz, $\left.\mathrm{CDCl}_{3}\right) \delta 207.3,160.0,154.1,141.0,130.2,116.7,114.2,110.5,93.4,84.1$, 81.2, 74.0, 55.41; IR (thin film) $v_{\max } 2963,1955,1805,1603,1586,1489,1434,1377,1325$, 1292, 1268, 1214, 1053, 940, 860, 767, 731, $699 \mathrm{~cm}^{-1}$; HRMS (ESI) calculated for $\mathrm{C}_{13} \mathrm{H}_{12} \mathrm{NaO}_{4}$ $[\mathrm{M}+\mathrm{Na}]^{+}$255.0628, found 255.0621.

\section{4-(4-methoxyphenyl)-4-(propa-1,2-dien-1-yl)-1,3-dioxolan-2-one (11)}<smiles>C=CC1(c2ccc(OC)cc2)COC(=O)O1</smiles>

Prepared according to the general procedure (method B) as described above from the starting material 2-bromoketone $\mathbf{l}$ (4.6 g, $20 \mathrm{mmol}$ ). The overall yield calculated based on amount of 2bromoketone 1: $7 \%$ yield $(290 \mathrm{mg})$. It was isolated by simple filtration and dried to afford a yellow solid. ${ }^{1} \mathrm{H}$ NMR (500 MHz, $\left.\mathrm{CDCl}_{3}\right) \delta 7.2(\mathrm{~d}, J=7.8 \mathrm{~Hz}, 2 \mathrm{H}), 6.9(\mathrm{~d}, J=7.8 \mathrm{~Hz}, 2 \mathrm{H}), 5.5$ (t, $J=6.7 \mathrm{~Hz}, 1 \mathrm{H}), 5.2-5.0(\mathrm{~m}, 2 \mathrm{H}), 4.7(\mathrm{~d}, J=8.5 \mathrm{~Hz}, 1 \mathrm{H}), 4.3(\mathrm{~d}, J=8.5 \mathrm{~Hz}, 1 \mathrm{H}), 3.8(\mathrm{~s}$, $3 \mathrm{H}) ;{ }^{13} \mathrm{C} \mathrm{NMR}\left(126 \mathrm{MHz}, \mathrm{CDCl}_{3}\right) \delta 206.3,159.0,153.1,130.2,125.2,113.3,92.4,83.2,80.0$, 73.1, 54.4; IR (thin film) $v_{\max } 2925,2322,2017,1682,1603,1512,1457,1275,1260,1180$, 1032, 832, 764, 750, 525, 495, 458, 447, 437, 430, $424 \mathrm{~cm}^{-1}$; HRMS (ESI) calculated for $\mathrm{C}_{13} \mathrm{H}_{12} \mathrm{NaO}_{4}[\mathrm{M}+\mathrm{Na}]^{+}$255.0628, found 255.0615. 


\section{4-([1,1'-biphenyl]-4-yl)-4-(propa-1,2-dien-1-yl)-1,3-dioxolan-2-one (1m)}

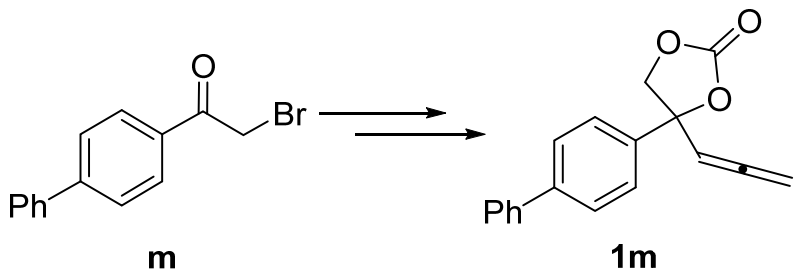

Prepared according to the general procedure (method B) as described above from the starting material 2-bromoketone $\mathbf{m}(5.5 \mathrm{~g}, 20 \mathrm{mmol})$. The overall yield calculated based on amount of 2-bromoketone $\mathbf{m}$ : 3\% yield $(170 \mathrm{mg})$. It was isolated by simple filtration and dried to afford a yellow solid (m.p. $\left.91-92{ }^{\circ} \mathrm{C}\right) .{ }^{1} \mathrm{H}$ NMR $\left(400 \mathrm{MHz}, \mathrm{CDCl}_{3}\right) \delta 7.68-7.59(\mathrm{~m}, 4 \mathrm{H}), 7.51-7.45$ (m, 4H), $7.42-7.37(\mathrm{~m}, 1 \mathrm{H}), 5.63(\mathrm{t}, J=6.6 \mathrm{~Hz}, 1 \mathrm{H}), 5.29-5.15(\mathrm{~m}, 2 \mathrm{H}), 4.89(\mathrm{~d}, J=8.5 \mathrm{~Hz}$ $1 \mathrm{H}), 4.44(\mathrm{~d}, J=8.5 \mathrm{~Hz}, 1 \mathrm{H}) ;{ }^{13} \mathrm{C} \mathrm{NMR}\left(101 \mathrm{MHz}, \mathrm{CDCl}_{3}\right) \delta 207.2,153.9,141.9,139.9,138.2$ $128.8,127.7,127.5,127.0,125.0,93.3,84.0,81.0,73.9$; IR (thin film) $v_{\max } 3031,1955,1804$, $1600,1487,1449,1377,1330,1258,1209,1187,1152,1120,1066,1007,841,766,752,734$ 698, 571, $504 \mathrm{~cm}^{-1}$; HRMS (ESI) calculated for $\mathrm{C}_{18} \mathrm{H}_{15} \mathrm{O}_{3}[\mathrm{M}+\mathrm{H}]^{+} 279.1027$, found 279.1018 .

\section{4-(naphthalen-2-yl)-4-(propa-1,2-dien-1-yl)-1,3-dioxolan-2-one (1n)}

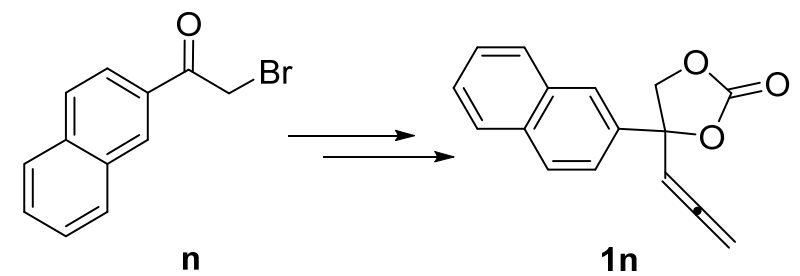

Prepared according to the general procedure (method B) as described above from the starting material 2-bromoketone $\mathbf{n}(5.0 \mathrm{~g}, 20 \mathrm{mmol})$. The overall yield calculated based on amount of 2-bromoketone $\mathbf{n}$ : $2 \%$ yield $(100 \mathrm{mg})$. It was isolated by simple filtration and dried to afford a yellow solid. ${ }^{1} \mathrm{H}$ NMR $\left(400 \mathrm{MHz}, \mathrm{CDCl}_{3}\right) \delta 7.9-7.9(\mathrm{~m}, 4 \mathrm{H}), 7.6-7.5(\mathrm{~m}, 2 \mathrm{H}), 7.4(\mathrm{dd}, J=$ 8.7, $1.9 \mathrm{~Hz}, 1 \mathrm{H}), 5.7(\mathrm{t}, J=6.6 \mathrm{~Hz}, 1 \mathrm{H}), 5.3-5.1(\mathrm{~m}, 2 \mathrm{H}), 4.9(\mathrm{~d}, J=8.4 \mathrm{~Hz}, 1 \mathrm{H}), 4.5(\mathrm{~d}, J=$ $8.4 \mathrm{~Hz}, 1 \mathrm{H}) ;{ }^{13} \mathrm{C}$ NMR $\left(101 \mathrm{MHz}, \mathrm{CDCl}_{3}\right) \delta 207.4,153.9,136.5,133.0,132.7,129.1,128.2$, 127.6, 126.8, 126.8, 123.8, 121.9, 93.3, 84.2, 81.0, 73.8; IR (thin film) $v_{\max } 3058,1954,1798$, $1601,1508,1473,1358,1325,1274,1186,1151,1058,1022,948,899,857,818,766,750$ 729, $477 \mathrm{~cm}^{-1}$; HRMS (ESI) calculated for $\mathrm{C}_{16} \mathrm{H}_{12} \mathrm{NaO}_{3}[\mathrm{M}+\mathrm{Na}]^{+} 275.0679$, found 275.0678. 


\section{4-methyl-4-(propa-1,2-dien-1-yl)-1,3-dioxolan-2-one (10)}

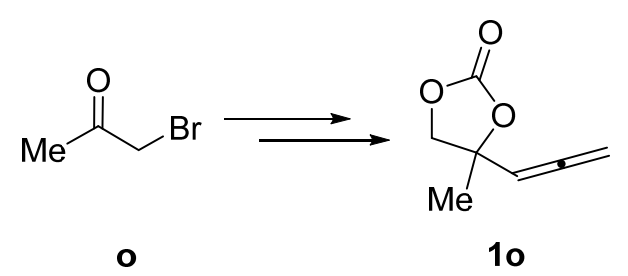

Prepared according to the general procedure $(\operatorname{method} A)$ as described above from the starting material 2-bromoketone $\mathbf{o}(2.7 \mathrm{~g}, 20 \mathrm{mmol})$. The overall yield calculated based on amount of 2-bromoketone $\mathbf{0}: 15 \%$ yield $(420 \mathrm{mg}$ ). It was isolated by simple filtration and dried to afford a yellow liquid. ${ }^{1} \mathrm{H} \mathrm{NMR}\left(300 \mathrm{MHz}, \mathrm{CDCl}_{3}\right) \delta 5.4(\mathrm{t}, J=6.6 \mathrm{~Hz}, 1 \mathrm{H}), 5.1(\mathrm{~d}, J=6.6 \mathrm{~Hz}, 2 \mathrm{H})$, $4.4(\mathrm{~d}, J=8.5 \mathrm{~Hz}, 1 \mathrm{H}), 4.2(\mathrm{~d}, J=8.5 \mathrm{~Hz}, 1 \mathrm{H}), 1.6(\mathrm{~s}, 3 \mathrm{H}) . ;{ }^{13} \mathrm{C} \mathrm{NMR}\left(126 \mathrm{MHz}, \mathrm{CDCl}_{3}\right) \delta$ 207.3, 154.3, 93.3, 81.4, 80.4, 74.0, 24.8., 111.9, 93.0, 83.5, 83.5, 81.2, 73.7; IR (thin film) $v_{\max }$ $2987,1957,1787,1480,1448,1389,1334,1283,1229,1181,1151,1093,1053,950,858,772$ 714, $609 \mathrm{~cm}^{-1}$; HRMS (ESI) calculated for $\mathrm{C}_{7} \mathrm{H}_{8} \mathrm{NaO}_{3}[\mathrm{M}+\mathrm{Na}]^{+}$163.0366, found 163.0368.

\section{4-ethyl-4-(propa-1,2-dien-1-yl)-1,3-dioxolan-2-one (1p)}

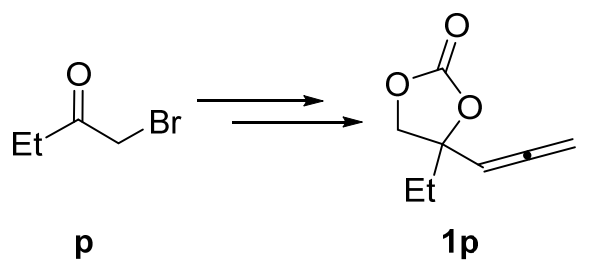

Prepared according to the general procedure (method A) as described above from the starting material 2-bromoketone $\mathbf{p}(3.0 \mathrm{~g}, 20 \mathrm{mmol})$. The overall yield calculated based on amount of 2-bromoketone p: $35 \%$ yield (1.08g). It was isolated by simple filtration and dried to afford a yellow liquid. ${ }^{1} \mathrm{H}$ NMR $\left(500 \mathrm{MHz}, \mathrm{CDCl}_{3}\right) \delta 5.3-5.3(\mathrm{~m}, 1 \mathrm{H}), 5.1-5.0(\mathrm{~m}, 2 \mathrm{H}), 4.4(\mathrm{dd}, J=$ 8.5, $1.3 \mathrm{~Hz}, 1 \mathrm{H}), 4.1(\mathrm{~d}, J=8.6 \mathrm{~Hz}, 1 \mathrm{H}), 1.9-1.8(\mathrm{~m}, 2 \mathrm{H}), 0.9(\mathrm{td}, J=7.5,1.5 \mathrm{~Hz}, 3 \mathrm{H}) ;{ }^{13} \mathrm{C}$ NMR $\left(126 \mathrm{MHz}, \mathrm{CDCl}_{3}\right) \delta 206.3,153.4,91.2,82.8,79.3,70.9,30.4,6.4$; IR (thin film) $v_{\max }$ $2977,1958,1798,1463,1382,1339,1274,1212,1178,1147,1108,1059,976,908,858,771$, 427, $408 \mathrm{~cm}^{-1}$; HRMS (ESI) calculated for $\mathrm{C}_{8} \mathrm{H}_{9} \mathrm{O}_{3}[\mathrm{M}-\mathrm{H}]^{-}$153.0557, found 153.0547. 


\section{4- cyclohexyl -4-(propa-1,2-dien-1-yl)-1,3-dioxolan-2-one (1q)}

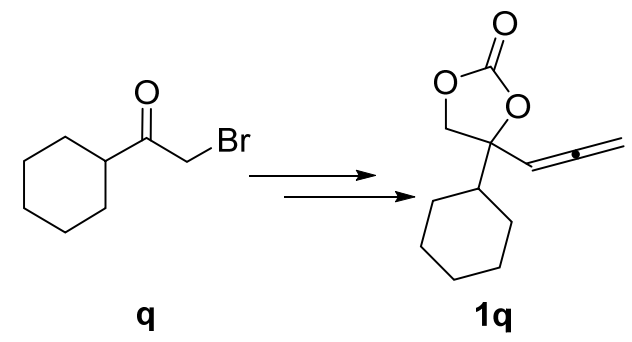

Prepared according to the general procedure (method A) as described above from the starting material 2-bromoketone $\mathbf{q}(4.1 \mathrm{~g}, 20 \mathrm{mmol})$. The overall yield calculated based on amount of 2-bromoketone q: $14 \%$ yield $(577 \mathrm{mg})$. It was isolated by simple filtration and dried to afford a yellow liquid. ${ }^{1} \mathrm{H}$ NMR (500 MHz, $\left.\mathrm{CDCl}_{3}\right) \delta 5.3(\mathrm{t}, J=6.7 \mathrm{~Hz}, 1 \mathrm{H}), 5.1-4.9(\mathrm{~m}, 2 \mathrm{H}), 4.4$ (dd, $J=8.6,1.2 \mathrm{~Hz}, 1 \mathrm{H}), 4.2(\mathrm{~d}, J=8.5 \mathrm{~Hz}, 1 \mathrm{H}), 1.8-1.5(\mathrm{~m}, 7 \mathrm{H}), 1.2-1.1(\mathrm{~m}, 2 \mathrm{H}), 1.0-0.9$ (m, 2H); ${ }^{13} \mathrm{C}$ NMR (126 MHz, Chloroform-d) $\delta 206.3,153.4,89.9,84.8,79.1,69.8,44.8,25.5$, 25.3, 25.0, 24.7, 24.6; IR (thin film) $v_{\max } 2930,2856,1957,1798,1452,1384,1336,1212,1174$, $1123,1086,1061,996,948,858,770,627,409 \mathrm{~cm}^{-1}$; HRMS (ESI) calculated for $\mathrm{C}_{12} \mathrm{H}_{15} \mathrm{O}_{3}$ [MH]207.1027, found 207.1019.

\section{4-(3-thienyl)-4-(propa-1,2-dien-1-yl)-1,3-dioxolan-2-one (1r)}

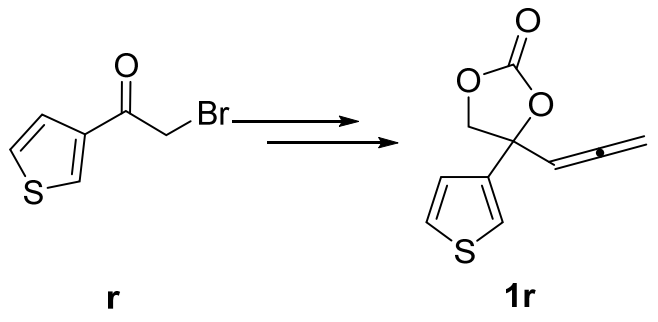

Prepared according to the general procedure (method A) as described above from the starting material 2-bromoketone $\mathbf{r}(4.1 \mathrm{~g}, 20 \mathrm{mmol})$. The overall yield calculated based on amount of 2bromoketone $\mathbf{r}$ : $15 \%$ yield $(450 \mathrm{mg})$. It was isolated by simple filtration and dried to afford a yellow liquid. ${ }^{1} \mathrm{H}$ NMR $\left(500 \mathrm{MHz}, \mathrm{CDCl}_{3}\right) \delta 7.4-7.4(\mathrm{~m}, 1 \mathrm{H}), 7.4-7.3(\mathrm{~m}, 1 \mathrm{H}), 7.1-7.0(\mathrm{~m}$, 1H), $5.6(\mathrm{t}, J=6.6 \mathrm{~Hz}, 1 \mathrm{H}), 5.2-5.1(\mathrm{~m}, 2 \mathrm{H}), 4.8(\mathrm{~d}, J=8.6 \mathrm{~Hz}, 1 \mathrm{H}), 4.4$ (d, $J=8.6 \mathrm{~Hz}, 1 \mathrm{H})$; ${ }^{13} \mathrm{C}$ NMR $\left(126 \mathrm{MHz}, \mathrm{CDCl}_{3}\right) \delta 207.2,154.0,140.3,127.8,124.7,122.7,92.8,82.4,81.2,73.7$; IR (thin film) $v_{\max } 3109,1955,1800,1474,1417,1375,1324,1255,1201,1152,1063,1010$, 940, 854, 791, 766, 726, 659, 528, 460, 453, 446, 420, $409 \mathrm{~cm}^{-1}$; HRMS (ESI) calculated for $\mathrm{C}_{10} \mathrm{H}_{7} \mathrm{O}_{3} \mathrm{~S}[\mathrm{M}-\mathrm{H}]^{-} 207.0121$, found 207.0114. 


\section{(2, 6-dichlorophenyl) formonitrile oxide (2b)}<smiles>[O-][N+]#Cc1c(Cl)cccc1Cl</smiles>

Prepared according to the general procedure as described above in 14\% yield (122 mg, 0.7 mmol). It was isolated by simple filtration and dried to afford a yellow solid (m.p. $172-173^{\circ} \mathrm{C}$ ). ${ }^{1} \mathrm{H} \mathrm{NMR}\left(400 \mathrm{MHz}, \mathrm{CDCl}_{3}\right) \delta 7.49-7.33(\mathrm{~m}, 3 \mathrm{H}) ;{ }^{13} \mathrm{C} \mathrm{NMR}\left(126 \mathrm{MHz}, \mathrm{CDCl}_{3}\right) \delta 137.3,130.5$, 127.7, 127.7, 127.0; IR (thin film) $v_{\max } 3083,2301,1680,1605,1574,1558,1488,1463,1434$, 1390, 1358, 1262, 1196, 1146, 1093, 1051, 990, 962, 910, 827, 793, 781, 763, 733, 708, 691, 649, 506, 457, $430 \mathrm{~cm}^{-1}$; HRMS (ESI) calculated for $\mathrm{C}_{7} \mathrm{H}_{2} \mathrm{Cl}_{2} \mathrm{NO}[\mathrm{M}-\mathrm{H}]^{-}$185.9519, found 185.9507.

\section{(2, 6-dibromophenyl) formonitrile oxide (2c)}

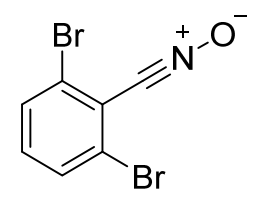

Prepared according to the general procedure as described above in $30 \%$ yield (400 $\mathrm{mg}, 1.5$ mmol). It was isolated by simple filtration and dried to afford a yellow solid (m.p. $243-244{ }^{\circ} \mathrm{C}$ ). ${ }^{1} \mathrm{H}$ NMR $\left(500 \mathrm{MHz}, \mathrm{CDCl}_{3}\right) \delta 7.53(\mathrm{~d}, J=8.1 \mathrm{~Hz}, 2 \mathrm{H}), 7.12(\mathrm{t}, J=8.1 \mathrm{~Hz}, 1 \mathrm{H}) ;{ }^{13} \mathrm{C} \mathrm{NMR}(126$ $\left.\mathrm{MHz}, \mathrm{CDCl}_{3}\right) \delta 131.0,130.6,126.0,119.2$; IR (thin film) $v_{\max } 3109,2478,2303,2181,2160$, 2019, 1975, 1564, 1543, 1455, 1425, 1352, 1261, 1184, 1131, 1031, 790, 778, 764, 726, 702, $564,525,494,458,445,438,430,424,408 \mathrm{~cm}^{-1}$; HRMS (ESI) calculated for $\mathrm{C}_{7} \mathrm{H}_{2} \mathrm{Br}_{2} \mathrm{NO}$ [MH]' 273.8498, found 273.8489.

\section{(2, 6-dimethoxyphenyl) formonitrile oxide (2d)}

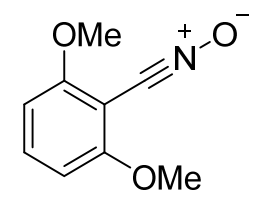

Prepared according to the general procedure as described above in $12 \%$ yield (116 mg, 0.6 mmol). It was isolated by simple filtration and dried to afford a yellow solid (m.p. $118-119{ }^{\circ} \mathrm{C}$ ). ${ }^{1} \mathrm{H}$ NMR (500 MHz, $\left.\mathrm{CDCl}_{3}\right) \delta 7.27(\mathrm{t}, J=8.5 \mathrm{~Hz}, 1 \mathrm{H}), 6.46(\mathrm{~d}, J=8.4 \mathrm{~Hz}, 2 \mathrm{H}), 3.80(\mathrm{~s}, 6 \mathrm{H})$; ${ }^{13} \mathrm{C}$ NMR $\left(126 \mathrm{MHz}, \mathrm{CDCl}_{3}\right) \delta 162.5,132.5,103.5,92.2,56.1$; IR (thin film) $v_{\max } 2947,2846$, 2399, 2308, 1592, 1479, 1457, 1433, 1341, 1303, 1262, 1107, 1059, 1025, 774, 721, 662, 533 $\mathrm{cm}^{-1}$; HRMS (ESI) calculated for $\mathrm{C}_{9} \mathrm{H}_{10} \mathrm{NO}_{3}[\mathrm{M}+\mathrm{H}]^{+} 180.0655$, found 180.0645 . 


\section{(2,4,5-trimethylphenyl) formonitrile oxide (2e)}<smiles>Cc1cc(C)c(C#[N+][O-])cc1C</smiles>

Prepared according to the general procedure as described above in 50\% yield (400 mg, 2.5 mmol). It was isolated by simple filtration and dried to afford a yellow liquid. ${ }^{1} \mathrm{H}$ NMR (400 $\left.\mathrm{MHz}, \mathrm{CDCl}_{3}\right) \delta 7.22(\mathrm{~s}, 1 \mathrm{H}), 7.04(\mathrm{~s}, 1 \mathrm{H}), 2.40(\mathrm{~s}, 3 \mathrm{H}), 2.26(\mathrm{~s}, 3 \mathrm{H}), 2.22(\mathrm{~s}, 3 \mathrm{H}) ;{ }^{13} \mathrm{C} \mathrm{NMR}$ $\left(126 \mathrm{MHz}, \mathrm{CDCl}_{3}\right) \delta 139.3,137.8,133.9,132.0,130.5,109.5,19.0,18.8,18.0$. IR (thin film) $v_{\max } 2922,2291,1590,1503,1451,1399,1373,1339,1275,1239,1196,1156,1032,994,910$, $876,733,649,446,430 \mathrm{~cm}^{-1}$; HRMS (ESI) calculated for $\mathrm{C}_{10} \mathrm{H}_{12} \mathrm{NO}[\mathrm{M}+\mathrm{H}]^{+} 162.0913$, found 162.0912

\section{(Z)-N-hydroxy-4-methylbenzimidoyl chloride (2f)}<smiles>Cc1ccc(/C(Cl)=N/O)cc1</smiles>

Prepared according to the general procedure as described above in $60 \%$ yield $(1.65 \mathrm{~g}, 12 \mathrm{mmol})$. It was isolated by simple filtration and dried to afford a gray solid (m.p. $70-71{ }^{\circ} \mathrm{C}$ ). ${ }^{1} \mathrm{H}$ NMR $\left(500 \mathrm{MHz}, \mathrm{CDCl}_{3}\right) \delta 8.3(\mathrm{~s}, 1 \mathrm{H}), 7.6(\mathrm{~d}, J=8.3 \mathrm{~Hz}, 2 \mathrm{H}), 7.1(\mathrm{~d}, J=8.4 \mathrm{~Hz}, 2 \mathrm{H}), 2.3(\mathrm{~s}, 3 \mathrm{H})$; ${ }^{13} \mathrm{C} \mathrm{NMR}\left(126 \mathrm{MHz}, \mathrm{CDCl}_{3}\right) \delta 140.2,139.5,128.6,128.2,126.1,20.3$; IR (thin film) $v_{\max } 3288$, $3049,2920,1625,1508,1449,1312,1241,1187,1000,941,848,816,792,711,673,596,480$, $416 \mathrm{~cm}^{-1}$; HRMS (ESI) calculated for $\mathrm{C}_{8} \mathrm{H}_{10} \mathrm{NO}[\mathrm{M}+\mathrm{H}]^{+}$136.0757, found 136.0757.

\section{(Z)-N-hydroxy-2-naphthimidoyl chloride (2g)}<smiles>O/N=C(\Cl)c1ccc2ccccc2c1</smiles>

Prepared according to the general procedure as described above in $13 \%$ yield $(431 \mathrm{mg}, 2.5$ mmol). It was isolated by simple filtration and dried to afford a gray solid (m.p. $100-101{ }^{\circ} \mathrm{C}$ ). ${ }^{1} \mathrm{H}$ NMR $\left(500 \mathrm{MHz}, \mathrm{CDCl}_{3}\right) \delta 8.3(\mathrm{~d}, J=1.8 \mathrm{~Hz}, 1 \mathrm{H}), 7.9-7.8(\mathrm{~m}, 3 \mathrm{H}), 7.8-7.7(\mathrm{~m}, 2 \mathrm{H}), 7.5$ - $7.4(\mathrm{~m}, 2 \mathrm{H}) ;{ }^{13} \mathrm{C}$ NMR $\left(126 \mathrm{MHz}, \mathrm{CDCl}_{3}\right) \delta 139.2,133.2,131.7,128.7,127.8,127.2,127.1$, 126.7, 126.5, 125.8, 122.4; IR (thin film) $v_{\max } 3361,3058,1603,1506,1353,1274,1261,1219$, 1187, 1127, 982, 927, 898, 861, 825, 749, 584, 473, 457, 445, 437, $423 \mathrm{~cm}^{-1}$; HRMS (ESI) calculated for $\mathrm{C}_{11} \mathrm{H}_{10} \mathrm{NO}[\mathrm{M}+\mathrm{H}]^{+}$172.0757, found 172.0757. 


\section{Characterization Data for All Products}

3-mesityl-6-phenyl-6-(propa-1,2-dien-1-yl)-5,6-dihydro-1,4,2-dioxazine (3aa)

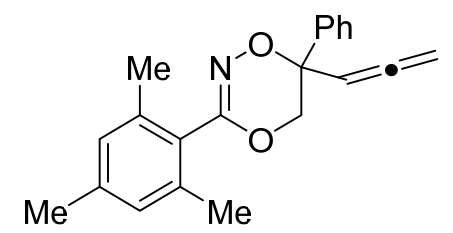

Prepared according to the general procedure (reaction time: $13 \mathrm{~h}$ ) as described above in $98 \%$ yield $(31.2 \mathrm{mg})$. It was purified by flash chromatography (3\% EtOAc/PE) to afford a yellow solid. ${ }^{1} \mathrm{H}$ NMR (500 MHz, $\left.\mathrm{CDCl}_{3}\right) \delta 7.53-7.48(\mathrm{~m}, 2 \mathrm{H}), 7.33(\mathrm{t}, J=7.6 \mathrm{~Hz}, 2 \mathrm{H}), 7.31-7.24$ (m, 1H), $6.74(\mathrm{~s}, 2 \mathrm{H}), 5.41(\mathrm{t}, J=6.7 \mathrm{~Hz}, 1 \mathrm{H}), 4.97-4.86(\mathrm{~m}, 2 \mathrm{H}), 4.45(\mathrm{~d}, J=2.0 \mathrm{~Hz}, 2 \mathrm{H})$, 2.18 (s, 3H), 2.07 (s, 6H); ${ }^{13} \mathrm{C}$ NMR (126 MHz, $\left.\mathrm{CDCl}_{3}\right) \delta$ 208.1, 154.0, 143.4, 139.5, 139.3, 137.5, 128.6, 128.2, 126.3, 93.3, 79.2, 75.5, 68.7, 21.2, 19.1; IR (thin film) $v_{\max } 2921,1955$, $1613,1491,1448,1375,1308,1295,1275,1261,1171,1097,1068,966,942,885,852,764$, 750, 700, 583, $423 \mathrm{~cm}^{-1}$; HRMS (ESI) calculated for $\mathrm{C}_{21} \mathrm{H}_{22} \mathrm{NO}_{2}[\mathrm{M}+\mathrm{H}]^{+} 320.1645$, found 320.1606 .

6-(2-fluorophenyl)-3-mesityl-6-(propa-1,2-dien-1-yl)-5,6-dihydro-1,4,2-dioxazine (3ba)

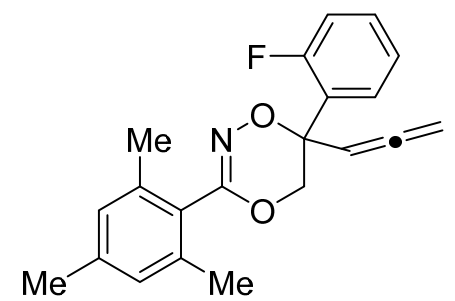

Prepared according to the general procedure (reaction time: $13 \mathrm{~h}$ ) as described above in $98 \%$ yield $(34.9 \mathrm{mg})$. It was purified by flash chromatography (3\% EtOAc/PE) to afford a yellow solid. ${ }^{1} \mathrm{H}$ NMR (400 MHz, $\left.\mathrm{CDCl}_{3}\right) \delta 7.77(\mathrm{td}, J=7.9,1.8 \mathrm{~Hz}, 1 \mathrm{H}), 7.40-7.30$ (m, 1H), 7.20 (td, $J=7.6,1.2 \mathrm{~Hz}, 1 \mathrm{H}), 7.09$ (ddd, $J=11.9,8.2,1.2 \mathrm{~Hz}, 1 \mathrm{H}), 6.81$ (s, 2H), 5.50 (t, $J=6.7 \mathrm{~Hz}$, $1 \mathrm{H}), 4.98(\mathrm{~d}, J=6.8 \mathrm{~Hz}, 1 \mathrm{H}), 4.97(\mathrm{~d}, J=6.5 \mathrm{~Hz}, 1 \mathrm{H}), 4.92(\mathrm{~d}, J=10.7 \mathrm{~Hz}, 1 \mathrm{H}), 4.57(\mathrm{dd}, J=$ 10.7, 1.9 Hz, 1H), 2.25 (s, 3H), 2.10 (s, 6H); $\left.{ }^{13} \mathrm{C} \mathrm{NMR} \mathrm{(101} \mathrm{MHz,} \mathrm{CDCl}_{3}\right) \delta$ 208.3, 154.2, 139.3, $137.3,130.1,130.0,129.3,129.3,128.1,127.5,124.2,124.1,116.1,115.9,91.9,91.9,79.2$, 74.4, 74.4, 68.0, 67.9, 21.0, 19.1; IR (thin film) $v_{\max } 2922,1955,1613,1487,1448,1376,1309$, $1275,1261,1220,1171,1098,1064,946,883,851,812,764,751,424 \mathrm{~cm}^{-1}$; HRMS (ESI) calculated for $\mathrm{C}_{21} \mathrm{H}_{21} \mathrm{FNO}_{2}[\mathrm{M}+\mathrm{H}]^{+}$338.1551, found 338.1512. 


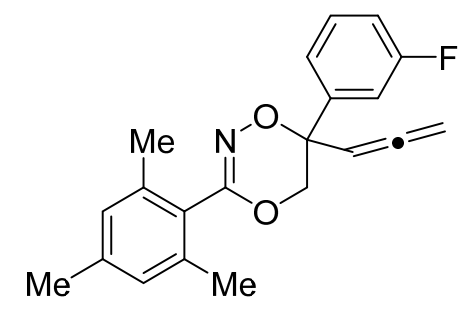

Prepared according to the general procedure (reaction time: $13 \mathrm{~h}$ ) as described above in $88 \%$ yield $(29.5 \mathrm{mg})$. It was purified by flash chromatography (3\% EtOAc/PE) to afford a yellow solid. ${ }^{1} \mathrm{H}$ NMR (400 MHz, $\left.\mathrm{CDCl}_{3}\right) \delta 7.45-7.29(\mathrm{~m}, 3 \mathrm{H}), 7.12-7.01(\mathrm{~m}, 1 \mathrm{H}), 6.84(\mathrm{~s}, 2 \mathrm{H})$, $5.48(\mathrm{t}, J=6.7 \mathrm{~Hz}, 1 \mathrm{H}), 5.04(\mathrm{dd}, J=11.0,5.9 \mathrm{~Hz}, 2 \mathrm{H}), 5.00(\mathrm{dd}, J=11.0,5.8 \mathrm{~Hz}, 1 \mathrm{H}), 4.54$ $(\mathrm{dd}, J=11.0 \mathrm{~Hz}, 1 \mathrm{H}), 4.51(\mathrm{dd}, J=11.0 \mathrm{~Hz}, 1 \mathrm{H}), 2.27(\mathrm{~s}, 3 \mathrm{H}), 2.16(\mathrm{~s}, 6 \mathrm{H}) ;{ }^{13} \mathrm{C}$ NMR $(101$ $\left.\mathrm{MHz}, \mathrm{CDCl}_{3}\right) \delta 208.1,164.0,161.6,154.0,141.9,141.9,139.4,137.3,130.0,130.0,128.1$, $127.5,121.9,121.8,115.3,115.1,113.8,113.5,92.9,79.3,75.1,75.0,68.3,21.0,19.0$; IR (thin film) $v_{\max } 2922,1955,1614,1591,1488,1443,1375,1308,1295,1275,1262,1170,1096,985$, 948, 901, 879, 852, 788, 764, 750, 695, 525, 457, $419 \mathrm{~cm}^{-1}$; HRMS (ESI) calculated for $\mathrm{C}_{21} \mathrm{H}_{21} \mathrm{FNO}_{2}[\mathrm{M}+\mathrm{H}]^{+}$338.1551, found 338.1522.

\section{6-(4-fluorophenyl)-3-mesityl-6-(propa-1,2-dien-1-yl)-5,6-dihydro-1,4,2-dioxazine (3da)}

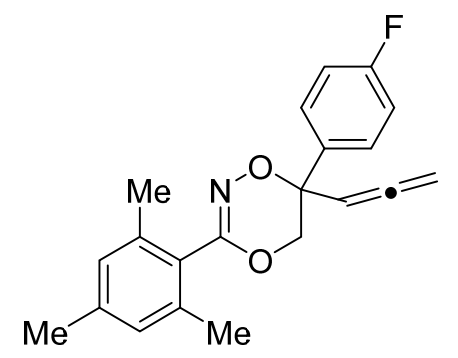

Prepared according to the general procedure (reaction time: $13 \mathrm{~h}$ ) as described above in $90 \%$ yield $(30.2 \mathrm{mg})$. It was purified by flash chromatography $(3 \% \mathrm{EtOAc} / \mathrm{PE})$ to afford a yellow solid. ${ }^{1} \mathrm{H}$ NMR (500 MHz, $\left.\mathrm{CDCl}_{3}\right) \delta 7.59-7.50(\mathrm{~m}, 2 \mathrm{H}), 7.13-7.04(\mathrm{~m}, 2 \mathrm{H}), 6.81(\mathrm{~s}, 2 \mathrm{H})$, $5.46(\mathrm{t}, J=6.7 \mathrm{~Hz}, 1 \mathrm{H}), 4.99(\mathrm{dd}, J=11.2,6.1 \mathrm{~Hz}, 1 \mathrm{H}), 4.96(\mathrm{dd}, J=11.2,6.1 \mathrm{~Hz}, 1 \mathrm{H}), 4.50$ (s, 2H), 2.25 (s, 3H), 2.14 (s, 6H); ${ }^{13} \mathrm{C}$ NMR (126 MHz, $\left.\mathrm{CDCl}_{3}\right) \delta 208.6,156.4,154.1,139.3$, 137.5, 129.6, 128.6, 128.2, 127.9, 126.5, 120.9, 111.4, 92.2, 78.6, 75.3, 68.1, 55.43, 21.2, 19.3; IR (thin film) $v_{\max } 2923,1955,1611,1509,1455,1376,1308,1295,1231,1161,1095,1070$, 1014, 966, 944, 884, 851, 836, 813, 750, 583, $540 \mathrm{~cm}^{-1}$; HRMS (ESI) calculated for $\mathrm{C}_{21} \mathrm{H}_{21} \mathrm{FNO}_{2}[\mathrm{M}+\mathrm{H}]^{+}$338.1551, found 338.1549. 


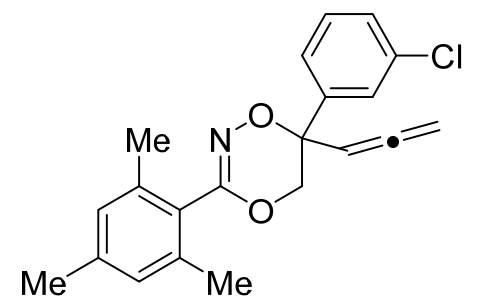

Prepared according to the general procedure (reaction time: $13 \mathrm{~h}$ ) as described above in $82 \%$ yield $(29.1 \mathrm{mg})$. It was purified by flash chromatography $(3 \% \mathrm{EtOAc} / \mathrm{PE})$ to afford a yellow solid. ${ }^{1} \mathrm{H}$ NMR $\left(500 \mathrm{MHz}, \mathrm{CDCl}_{3}\right) \delta 7.69-7.62(\mathrm{~m}, 1 \mathrm{H}), 7.47-7.37(\mathrm{~m}, 2 \mathrm{H}), 7.23-7.17$ (m, 1H), $6.75(\mathrm{~s}, 2 \mathrm{H}), 5.38(\mathrm{t}, J=6.7 \mathrm{~Hz}, 1 \mathrm{H}), 5.00-4.88(\mathrm{~m}, 2 \mathrm{H}), 4.43(\mathrm{~s}, 2 \mathrm{H}), 2.18(\mathrm{~s}, 3 \mathrm{H}), 2.07$ (s, 6H); ${ }^{13} \mathrm{C}$ NMR $\left(126 \mathrm{MHz}, \mathrm{CDCl}_{3}\right) \delta 207.2,153.1,140.6,138.6,136.4,130.5,129.1,128.6$, $127.2,126.5,124.1,121.7,91.9,78.5,74.1,67.4,20.1,18.1$; IR (thin film) $v_{\max } 2921,1954$ $1612,1567,1418,1375,1308,1294,1261,1170,1093,1072,996,945,882,850,787,750$, 693, 661, $584 \mathrm{~cm}^{-1}$; HRMS (ESI) calculated for $\mathrm{C}_{21} \mathrm{H}_{21} \mathrm{ClNO}_{2}[\mathrm{M}+\mathrm{H}]^{+}$354.1255, found 354.1254 .

\section{6-(4-chlorophenyl)-3-mesityl-6-(propa-1,2-dien-1-yl)-5,6-dihydro-1,4,2-dioxazine (3fa)}

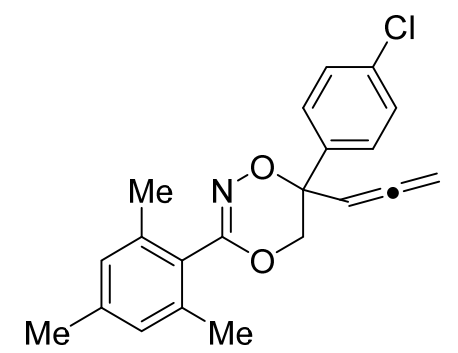

Prepared according to the general procedure (reaction time: $13 \mathrm{~h}$ ) as described above in $98 \%$ yield $(35.5 \mathrm{mg})$. It was purified by flash chromatography $(3 \% \mathrm{EtOAc} / \mathrm{PE})$ to afford a yellow solid. ${ }^{1} \mathrm{H}$ NMR (500 MHz, $\left.\mathrm{CDCl}_{3}\right) \delta 7.44(\mathrm{~d}, J=8.6 \mathrm{~Hz}, 2 \mathrm{H}), 7.30$ (d, $\left.J=8.5 \mathrm{~Hz}, 2 \mathrm{H}\right), 6.74(\mathrm{~s}$, 2H), $5.38(\mathrm{t}, J=6.7 \mathrm{~Hz}, 1 \mathrm{H}), 4.98-4.85(\mathrm{~m}, 2 \mathrm{H}), 4.42(\mathrm{~d}, J=10.9 \mathrm{~Hz}, 1 \mathrm{H}), 4.41(\mathrm{~d}, J=10.9$ $\mathrm{Hz}, 1 \mathrm{H}), 2.22-2.13(\mathrm{~m}, 3 \mathrm{H}), 2.06(\mathrm{~s}, 6 \mathrm{H}) ;{ }^{13} \mathrm{C} \mathrm{NMR}\left(126 \mathrm{MHz}, \mathrm{CDCl}_{3}\right) \delta 207.2,153.1,138.6$, $136.8,136.4,133.3,127.7,127.2,126.9,126.5,92.0,78.4,74.2,67.4,20.1,18.1 ;$ IR (thin film) $v_{\max } 2924,1613,1491,1456,1309,1275,1261,1171,1093,1013,946,851,827,764,750,458$, 437, $419 \mathrm{~cm}^{-1}$; HRMS (ESI) calculated for $\mathrm{C}_{21} \mathrm{H}_{21} \mathrm{ClNO}_{2}[\mathrm{M}+\mathrm{H}]^{+}$354.1255, found 354.1213. 
6-(3-bromophenyl)-3-mesityl-6-(propa-1,2-dien-1-yl)-5,6-dihydro-1,4,2-dioxazine (3ga)

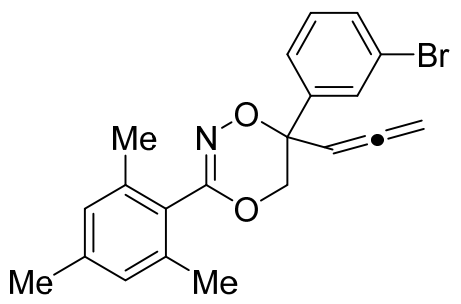

Prepared according to the general procedure (reaction time: $13 \mathrm{~h}$ ) as described above in $83 \%$ yield $(32.9 \mathrm{mg})$. It was purified by flash chromatography $(3 \%$ EtOAc/PE) to afford a yellow solid. ${ }^{1} \mathrm{H}$ NMR $\left(500 \mathrm{MHz}, \mathrm{CDCl}_{3}\right) \delta 7.53-7.48(\mathrm{~m}, 1 \mathrm{H}), 7.41-7.35(\mathrm{~m}, 1 \mathrm{H}), 7.31-7.23(\mathrm{~m}$, 2H), 6.74 (s, 2H), 5.38 (t, J=6.7 Hz, 1H), $4.98-4.90$ (m, 2H), 4.43 (s, 2H), 2.18 (s, 3H), 2.07 (s, 6H); ${ }^{13} \mathrm{C}$ NMR $\left(126 \mathrm{MHz}, \mathrm{CDCl}_{3}\right) \delta 207.2,153.1,140.4,138.6,136.4,133.6,128.9,127.5$, $127.2,126.5,125.8,123.6,91.9,78.5,74.2,67.4,20.1,18.1$; IR (thin film) $v_{\max } 2922,1955$, $1612,1574,1456,1375,1308,1295,1275,1261,1170,1094,1032,946,884,851,791,764$, 750, 694, $420 \mathrm{~cm}^{-1}$; HRMS (ESI) calculated for $\mathrm{C}_{21} \mathrm{H}_{21} \mathrm{BrNO}_{2}[\mathrm{M}+\mathrm{H}]^{+}$398.0750, found 398.0748

6-(4-bromophenyl)-3-mesityl-6-(propa-1,2-dien-1-yl)-5,6-dihydro-1,4,2-dioxazine (3ha)

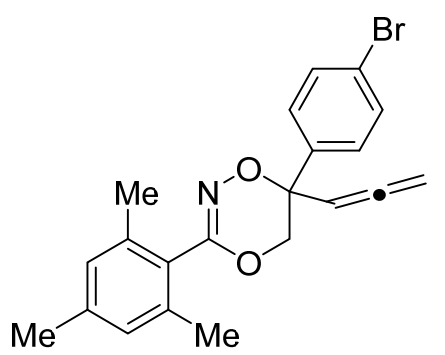

Prepared according to the general procedure (reaction time: $13 \mathrm{~h}$ ) as described above in $94 \%$ yield $(37.3 \mathrm{mg})$. It was purified by flash chromatography (3\% EtOAc/PE) to afford a yellow solid. ${ }^{1} \mathrm{H}$ NMR (500 MHz, $\left.\mathrm{CDCl}_{3}\right) \delta 7.46(\mathrm{~d}, J=8.5 \mathrm{~Hz}, 2 \mathrm{H}), 7.38(\mathrm{~d}, J=8.5 \mathrm{~Hz}, 2 \mathrm{H}), 6.74(\mathrm{~s}$, 2H), $5.37(\mathrm{t}, J=6.7 \mathrm{~Hz}, 1 \mathrm{H}), 4.97-4.86(\mathrm{~m}, 2 \mathrm{H}), 4.43(\mathrm{~d}, J=10.9 \mathrm{~Hz}, 1 \mathrm{H}), 4.40(\mathrm{~d}, J=10.9$ $\mathrm{Hz}, 1 \mathrm{H}), 2.18(\mathrm{~s}, 3 \mathrm{H}), 2.07$ (s, 6H); ${ }^{13} \mathrm{C} \mathrm{NMR}\left(126 \mathrm{MHz}, \mathrm{CDCl}_{3}\right) \delta 207.2,153.1,138.6,137.4$, $136.4,130.7,127.2,127.2,126.5,121.5,92.0,78.4,74.2,67.4,20.1,18.1$; IR (thin film) $v_{\max }$ $2922,1955,1612,1487,1455,1395,1308,1294,1261,1170,1095,1075,1009,966,946,884$, 851, 822, $750 \mathrm{~cm}^{-1}$; HRMS (ESI) calculated for $\mathrm{C}_{21} \mathrm{H}_{21} \mathrm{BrNO}_{2}[\mathrm{M}+\mathrm{H}]^{+}$398.0750, found 398.0722 


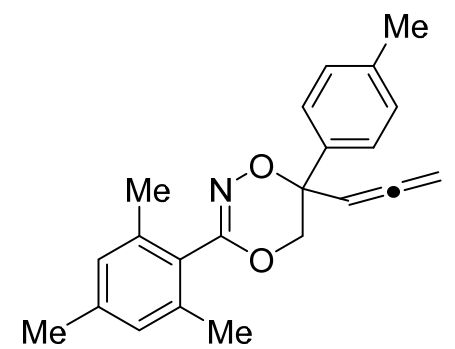

Prepared according to the general procedure (reaction time: $13 \mathrm{~h}$ ) as described above in $98 \%$ yield $(33.7 \mathrm{mg})$. It was purified by flash chromatography $(3 \% \mathrm{EtOAc} / \mathrm{PE})$ to afford a yellow solid. ${ }^{1} \mathrm{H}$ NMR $\left(500 \mathrm{MHz}, \mathrm{CDCl}_{3}\right) \delta 7.38(\mathrm{~d}, J=8.1 \mathrm{~Hz}, 2 \mathrm{H}), 7.13(\mathrm{~d}, J=7.9 \mathrm{~Hz}, 2 \mathrm{H}), 6.74(\mathrm{~s}$, 2H), $5.40(\mathrm{t}, J=6.7 \mathrm{~Hz}, 1 \mathrm{H}), 4.93(\mathrm{dd}, J=11.6,6.9 \mathrm{~Hz}, 2 \mathrm{H}), 4.88(\mathrm{dd}, J=11.5,6.6 \mathrm{~Hz}, 1 \mathrm{H})$, $4.44(\mathrm{~d}, J=10.8 \mathrm{~Hz}, 1 \mathrm{H}), 4.38(\mathrm{~d}, J=10.8 \mathrm{~Hz}, 1 \mathrm{H}), 2.31-2.27$ (m, 3H), 2.18 (s, 3H), $2.11-$ $2.08(\mathrm{~m}, 6 \mathrm{H}) ;{ }^{13} \mathrm{C} \mathrm{NMR}\left(101 \mathrm{MHz}, \mathrm{CDCl}_{3}\right) \delta 208.0,153.8,139.2,138.1,137.4,136.3,129.1$, $128.1,127.7,126.1,93.3,78.9,75.2,68.6,21.0,21.0,19.0$; IR (thin film) $v_{\max } 2922,1956,1613$, $1512,1455,1375,1309,1295,1276,1261,1170,1095,1071,966,945,886,851,816,764$, $750,583 \mathrm{~cm}^{-1}$; HRMS (ESI) calculated for $\mathrm{C}_{22} \mathrm{H}_{24} \mathrm{NO}_{2}[\mathrm{M}+\mathrm{H}]^{+} 334.1802$, found 334.1756.

\section{3-mesityl-6-(2-methoxyphenyl)-6-(propa-1,2-dien-1-yl)-5,6-dihydro-1,4,2-dioxazine (3ja)}

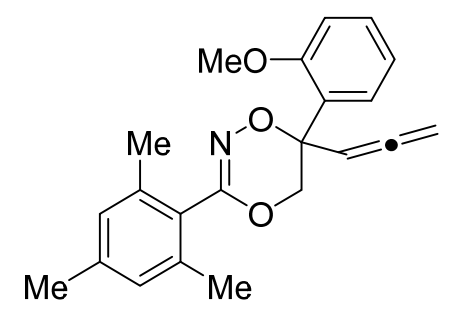

Prepared according to the general procedure (reaction time: $13 \mathrm{~h}$ ) as described above in $93 \%$ yield $(32.4 \mathrm{mg})$. It was purified by flash chromatography (3\% EtOAc/PE) to afford a yellow solid. ${ }^{1} \mathrm{H}$ NMR (500 MHz, $\left.\mathrm{CDCl}_{3}\right) \delta 7.74(\mathrm{dd}, J=7.8,1.8 \mathrm{~Hz}, 1 \mathrm{H}), 7.31$ (ddd, $J=8.1,7.3,1.7$ $\mathrm{Hz}, 1 \mathrm{H}), 7.00$ (ddd, $J=7.8,7.3,1.1 \mathrm{~Hz}, 1 \mathrm{H}), 6.91(\mathrm{dd}, J=8.2,1.1 \mathrm{~Hz}, 1 \mathrm{H}), 6.78$ (s, 2H), 5.51 (t, $J=6.7 \mathrm{~Hz}, 1 \mathrm{H}), 5.02(\mathrm{~d}, J=10.4 \mathrm{~Hz}, 1 \mathrm{H}), 4.91(\mathrm{~d}, J=6.7 \mathrm{~Hz}, 2 \mathrm{H}), 4.58(\mathrm{~d}, J=10.3 \mathrm{~Hz}$, 1H), $3.84(\mathrm{~s}, 3 \mathrm{H}), 2.23(\mathrm{~s}, 3 \mathrm{H}) ;{ }^{13} \mathrm{C} \mathrm{NMR}\left(126 \mathrm{MHz}, \mathrm{CDCl}_{3}\right) \delta$ 208.1, 159.9, 154.1, 141.0, 139.5, $137.5,129.6,128.3,127.8,118.5,114.0,112.0,93.3,79.2,75.4,68.7,55.4,21.2,19.2$; IR (thin film) $v_{\max } 2923,1956,1613,1584,1489,1458,1436,1368,1309,1294,1244,1170,1127,1097$, 1063, 1025, 950, 884, 851, 794, 757, 588, 426, 415, $403 \mathrm{~cm}^{-1}$; HRMS (ESI) calculated for $\mathrm{C}_{22} \mathrm{H}_{24} \mathrm{NO}_{3}[\mathrm{M}+\mathrm{H}]^{+} 350.1751$, found 350.1701 . 


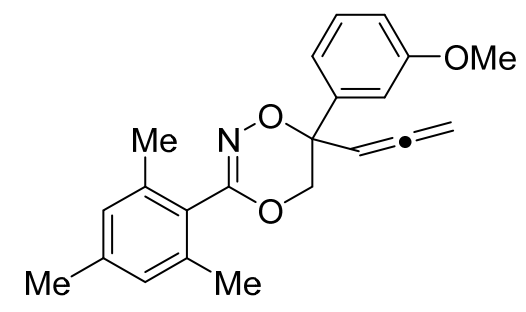

Prepared according to the general procedure (reaction time: $13 \mathrm{~h}$ ) as described above in $91 \%$ yield $(31.7 \mathrm{mg})$. It was purified by flash chromatography $(3 \%$ EtOAc/PE) to afford a yellow solid. ${ }^{1} \mathrm{H}$ NMR (500 MHz, $\left.\mathrm{CDCl}_{3}\right) \delta 7.31$ (t, $\left.J=8.0 \mathrm{~Hz}, 1 \mathrm{H}\right), 7.15$ (t, $\left.J=2.0 \mathrm{~Hz}, 1 \mathrm{H}\right), 7.15-$ 7.09 (m, 1H), 6.88 (ddd, $J=8.1,2.7,1.0 \mathrm{~Hz}, 1 \mathrm{H}), 6.82(\mathrm{~s}, 2 \mathrm{H}), 5.47$ (t, $J=6.7 \mathrm{~Hz}, 1 \mathrm{H}), 5.01$ (dd, $J=11.7,6.8 \mathrm{~Hz}, 2 \mathrm{H}), 4.97(\mathrm{dd}, J=11.6,6.7 \mathrm{~Hz}, 1 \mathrm{H}), 4.53(\mathrm{~d}, J=10.8 \mathrm{~Hz}, 1 \mathrm{H}), 4.47$ (d, $J$ $=10.8 \mathrm{~Hz}, 1 \mathrm{H}), 3.83(\mathrm{~s}, 3 \mathrm{H}), 2.25(\mathrm{~s}, 3 \mathrm{H}), 2.17(\mathrm{~s}, 6 \mathrm{H}) ;{ }^{13} \mathrm{C} \mathrm{NMR}\left(126 \mathrm{MHz}, \mathrm{CDCl}_{3}\right) \delta 208.2$, 163.7, 161.7, 154.0, 139.6, 137.4, 135.1, 135.1, 128.4, 128.4, 128.3, 127.6, 115.6, 115.4, 93.2, 79.3, 75.2, 68.6, 21.2, 19.1; IR (thin film) $v_{\max } 2921,1955,1610,1584,1486,1431,1375,1308$, 1290, 1267, 1169, 1094, 1072, 1032, 976, 947, 850, 783, 741, 698, $540 \mathrm{~cm}^{-1}$; HRMS (ESI) calculated for $\mathrm{C}_{22} \mathrm{H}_{24} \mathrm{NO}_{3}[\mathrm{M}+\mathrm{H}]^{+} 350.1751$, found 350.1704 .

\section{3-mesityl-6-(4-methoxyphenyl)-6-(propa-1,2-dien-1-yl)-5,6-dihydro-1,4,2-dioxazine (3la)}

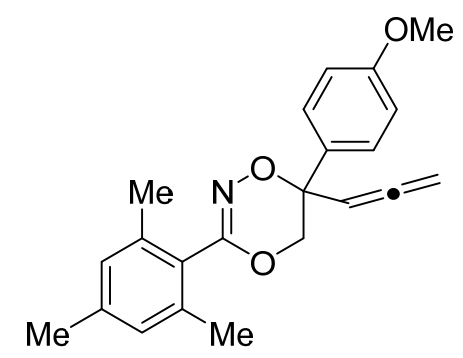

Prepared according to the general procedure (reaction time: $13 \mathrm{~h}$ ) as described above in $98 \%$ yield $(34.2 \mathrm{mg})$. It was purified by flash chromatography (3\% EtOAc/PE) to afford a yellow solid. ${ }^{1} \mathrm{H}$ NMR (500 MHz, $\left.\mathrm{CDCl}_{3}\right) \delta 7.41$ (d, $\left.J=7.5 \mathrm{~Hz}, 3 \mathrm{H}\right), 6.85$ (d, J=7.6 Hz, 2H), 6.74 (s, 2H), $5.43-5.36(\mathrm{~m}, 1 \mathrm{H}), 4.96-4.84(\mathrm{~m}, 2 \mathrm{H}), 4.42(\mathrm{~d}, J=10.7 \mathrm{~Hz}, 1 \mathrm{H}), 4.38(\mathrm{~d}, J=10.8 \mathrm{~Hz}$, 2H), 3.74 (s, 3H), 2.18 (s, 3H), 2.10 (s, 6H); $\left.{ }^{13} \mathrm{C} \mathrm{NMR} \mathrm{(126} \mathrm{MHz,} \mathrm{CDCl}_{3}\right) \delta$ 207.0, 158.6, 152.8, $138.4,136.5,130.3,127.2,126.8,126.7,112.9,92.3,78.0,74.2,67.7,54.3,20.1,18.2$; IR (thin film) $v_{\max } 2961,1954,1610,1511,1455,1306,1249,1177,1092,1024,964,850,828,797$, 741, 701, 584, $544 \mathrm{~cm}^{-1}$; HRMS (ESI) calculated for $\mathrm{C}_{22} \mathrm{H}_{24} \mathrm{NO}_{3}[\mathrm{M}+\mathrm{H}]^{+}$350.1751, found 350.1747 . 


\section{6-([1,1'-biphenyl]-4-yl)-3-mesityl-6-(propa-1,2-dien-1-yl)-5,6-dihydro-1,4,2-dioxazine}

\section{(3ma)}

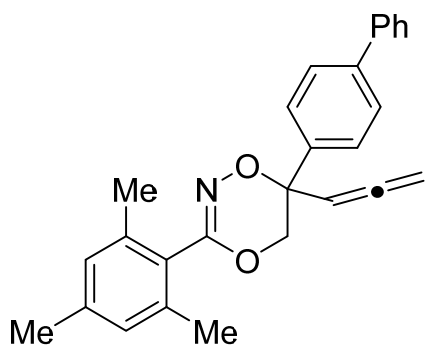

Prepared according to the general procedure (reaction time: $13 \mathrm{~h}$ ) as described above in $71 \%$ yield $(28.1 \mathrm{mg})$. It was purified by flash chromatography (3\% EtOAc/PE) to afford a yellow solid. ${ }^{1} \mathrm{H}$ NMR (500 MHz, $\left.\mathrm{CDCl}_{3}\right) \delta 7.60-7.49(\mathrm{~m}, 6 \mathrm{H}), 7.41-7.34(\mathrm{~m}, 2 \mathrm{H}), 7.33$ - $7.26(\mathrm{~m}$, 1H), $6.74(\mathrm{~s}, 2 \mathrm{H}), 5.45(\mathrm{t}, J=6.7 \mathrm{~Hz}, 1 \mathrm{H}), 4.96(\mathrm{dd}, J=11.7,6.7 \mathrm{~Hz}, 2 \mathrm{H}), 4.92$ (dd, $J=11.7$, $6.7 \mathrm{~Hz}, 1 \mathrm{H}), 4.50(\mathrm{~d}, J=10.9 \mathrm{~Hz}, 2 \mathrm{H}), 4.47(\mathrm{~d}, J=11.0 \mathrm{~Hz}, 1 \mathrm{H}), 2.17(\mathrm{~s}, 3 \mathrm{H}), 2.09(\mathrm{~s}, 6 \mathrm{H}),{ }^{13} \mathrm{C}$ NMR $\left(101 \mathrm{MHz}, \mathrm{CDCl}_{3}\right) \delta 208.0,153.9,141.2,140.4,139.3,138.3,137.4,128.7,128.1,127.6$, $127.4,127.1,127.0,126.7,93.2,79.1,75.3,68.5,21.0,19.0$; IR (thin film) $v_{\max } 3735,2923$, $1955,1613,1487,1456,1309,1275,1261,1170,1095,965,851,765,750,699,567,507,457$, 446, 437, 430, $419 \mathrm{~cm}^{-1}$; HRMS (ESI) calculated for $\mathrm{C}_{27} \mathrm{H}_{26} \mathrm{NO}_{2}[\mathrm{M}+\mathrm{H}]^{+} 396.1958$, found 396.1953 .

\section{3-mesityl-6-(naphthalen-1-yl)-6-(propa-1,2-dien-1-yl)-5,6-dihydro-1,4,2-dioxazine (3na)}

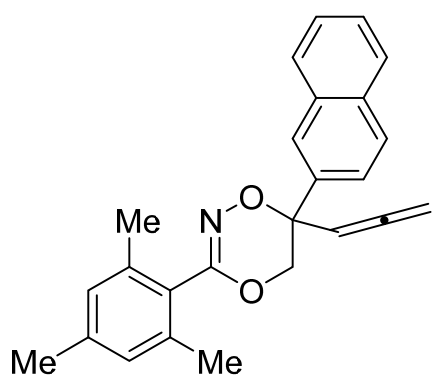

Prepared according to the general procedure (reaction time: $13 \mathrm{~h}$ ) as described above in $78 \%$ yield $(28.6 \mathrm{mg})$. It was purified by flash chromatography ( $3 \%$ EtOAc/PE) to afford a yellow solid. ${ }^{1} \mathrm{H}$ NMR (500 MHz, $\left.\mathrm{CDCl}_{3}\right) \delta 8.07(\mathrm{~s}, 1 \mathrm{H}), 7.90-7.82(\mathrm{~m}, 3 \mathrm{H}), 7.68-7.61(\mathrm{~m}, 1 \mathrm{H})$, $7.54-7.47(\mathrm{~m}, 2 \mathrm{H}), 6.79$ (s, 2H), $5.56(\mathrm{t}, J=6.7 \mathrm{~Hz}, 1 \mathrm{H}), 5.03(\mathrm{dd}, J=11.8,6.9 \mathrm{~Hz}, 1 \mathrm{H}), 4.99$ (dd, $J=11.7,6.6 \mathrm{~Hz}, 1 \mathrm{H}), 4.67-4.57$ (m, 2H), 2.23 (s, 3H), $2.12(\mathrm{~s}, 6 \mathrm{H}) ;{ }^{13} \mathrm{C}$ NMR $(101 \mathrm{MHz}$, $\left.\mathrm{CDCl}_{3}\right) \delta 208.1,154.0,139.3,137.4,136.5,133.0,133.0,128.3,128.2,128.1,127.6,127.5$, 126.4, 126.2, 125.6, 123.7, 93.2, 79.1, 75.5, 68.5, 21.0, 19.0; IR (thin film) $v_{\max } 2923,1955$, $1730,1613,1507,1455,1355,1308,1294,1275,1261,1171,1094,982,935,890,852,818$, 764, 750, 581, 478, 457, $423 \mathrm{~cm}^{-1}$; HRMS (ESI) calculated for $\mathrm{C}_{25} \mathrm{H}_{24} \mathrm{NO}_{2}[\mathrm{M}+\mathrm{H}]^{+} 370.1802$, found 370.1762 . 


\section{3-mesityl-6-methyl-6-(propa-1,2-dien-1-yl)-5,6-dihydro-1,4,2-dioxazine (3oa)}

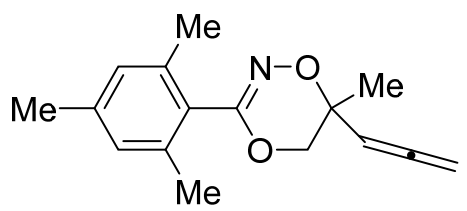

Prepared according to the general procedure (reaction time: $5 \mathrm{~h}$ ) as described above in $98 \%$ yield $(25.2 \mathrm{mg})$. It was isolated by simple filtration and dried to afford a white liquid. ${ }^{1} \mathrm{H}$ NMR $\left(500 \mathrm{MHz}, \mathrm{CDCl}_{3}\right) \delta 6.8(\mathrm{~s}, 2 \mathrm{H}), 5.3(\mathrm{t}, J=6.7 \mathrm{~Hz}, 1 \mathrm{H}), 4.9-4.8(\mathrm{~m}, 2 \mathrm{H}), 4.2(\mathrm{~d}, J=10.5 \mathrm{~Hz}$, $1 \mathrm{H}), 4.0(\mathrm{~d}, J=10.4 \mathrm{~Hz}, 1 \mathrm{H}), 2.3(\mathrm{~s}, 6 \mathrm{H}), 2.2(\mathrm{~s}, 3 \mathrm{H}), 1.4(\mathrm{~s}, 3 \mathrm{H}) ;{ }^{13} \mathrm{C} \mathrm{NMR}\left(126 \mathrm{MHz}, \mathrm{CDCl}_{3}\right)$ $\delta 206.3,152.6,138.4,136.4,127.3,126.9,92.2,77.8,76.2,70.2,68.4,21.7,21.7,20.1,18.4$ IR (thin film) $v_{\max } 2922,1956,1611,1455,1372,1308,1295,1274,1170,1122,1082,1012$, 992, 955, 888, 849, 802, 740, 700, 572, $530 \mathrm{~cm}^{-1}$; HRMS (ESI) calculated for $\mathrm{C}_{16} \mathrm{H}_{20} \mathrm{NO}_{2}$ $[\mathrm{M}+\mathrm{H}]^{+} 258.1489$, found 258.1491 .

\section{3-mesityl-6-ethyl-6-(propa-1,2-dien-1-yl)-5,6-dihydro-1,4,2-dioxazine (3pa)}

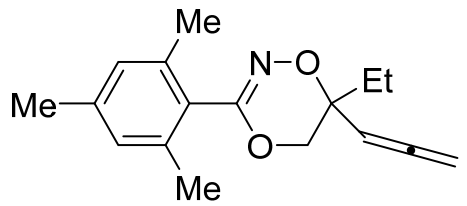

Prepared according to the general procedure (reaction time: $6 \mathrm{~h}$ ) as described above in $83 \%$ yield (22.3 mg). It was isolated by simple filtration and dried to afford a yellow liquid. ${ }^{1} \mathrm{H}$ NMR $\left(500 \mathrm{MHz}, \mathrm{CDCl}_{3}\right) \delta 6.8(\mathrm{~s}, 2 \mathrm{H}), 5.2(\mathrm{td}, J=6.7,1.2 \mathrm{~Hz}, 1 \mathrm{H}), 4.9-4.8(\mathrm{~m}, 2 \mathrm{H}), 4.3(\mathrm{~d}, J=$ $10.4 \mathrm{~Hz}, 1 \mathrm{H}), 4.0(\mathrm{dd}, J=10.5,1.2 \mathrm{~Hz}, 1 \mathrm{H}), 2.3(\mathrm{~s}, 6 \mathrm{H}), 2.2(\mathrm{~s}, 3 \mathrm{H}), 1.8-1.7(\mathrm{~m}, 1 \mathrm{H}), 1.7-$ $1.6(\mathrm{~m}, 1 \mathrm{H}), 1.0(\mathrm{t}, J=7.6 \mathrm{~Hz}, 3 \mathrm{H}) ;{ }^{13} \mathrm{C} \mathrm{NMR}\left(126 \mathrm{MHz}, \mathrm{CDCl}_{3}\right) \delta 206.7,152.9,138.4,136.4$, 127.3, 127.0, 90.7, 77.4, 73.0, 67.2, 28.3, 20.1, 18.4, 6.2; IR (thin film) $v_{\max } 2972,1612,1457$, $1309,1294,1171,1081,1031,961,933,894,850,740,581,528,491,473,460,446,427,409$ $\mathrm{cm}^{-1}$; HRMS (ESI) calculated for $\mathrm{C}_{17} \mathrm{H}_{22} \mathrm{O}_{2} \mathrm{~N}[\mathrm{M}+\mathrm{H}]^{+} 272.1645$, found 272.1641 . 
3-mesityl-6-cyclohexyl-6-(propa-1,2-dien-1-yl)-5,6-dihydro-1,4,2-dioxazine (3qa)

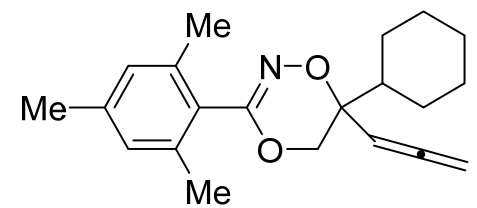

Prepared according to the general procedure (reaction time: $36 \mathrm{~h}$ ) as described above in 58\% yield (19 mg). It was isolated by simple filtration and dried to afford a white liquid. ${ }^{1} \mathrm{H}$ NMR $\left(500 \mathrm{MHz}, \mathrm{CDCl}_{3}\right) \delta 6.8(\mathrm{~s}, 2 \mathrm{H}), 5.2(\mathrm{td}, J=6.7,1.4 \mathrm{~Hz}, 1 \mathrm{H}), 4.8(\mathrm{qd}, J=11.3,6.8 \mathrm{~Hz}, 2 \mathrm{H})$, $4.3(\mathrm{~d}, J=10.4 \mathrm{~Hz}, 1 \mathrm{H}), 4.0(\mathrm{dd}, J=10.4,1.5 \mathrm{~Hz}, 1 \mathrm{H}), 2.2(\mathrm{~s}, 6 \mathrm{H}), 2.2(\mathrm{~s}, 3 \mathrm{H}), 1.8-1.6(\mathrm{~m}$, 5H), $1.5(\mathrm{~s}, 1 \mathrm{H}), 1.2-1.0(\mathrm{~m}, 5 \mathrm{H}) ;{ }^{13} \mathrm{C} \mathrm{NMR}\left(126 \mathrm{MHz}, \mathrm{CDCl}_{3}\right) \delta 207.1,153.2,138.3,136.4$, $127.2,127.1,89.4,76.9,76.2,74.7,66.6,43.6,25.5,25.3,25.1,25.1,20.1,18.3$; IR (thin film) $v_{\max } 2928,2855,1613,1452,1314,1264,1087,981,906,849,801,729,649,581,527,467$, 460, 446, 427, 420, $414 \mathrm{~cm}^{-1}$; HRMS (ESI) calculated for $\mathrm{C}_{21} \mathrm{H}_{28} \mathrm{O}_{2} \mathrm{~N}[\mathrm{M}+\mathrm{H}]^{+} 326.2115$, found 326.2101 .

\section{3-mesityl-6-(3-thienyl)-6-(propa-1,2-dien-1-yl)-5,6-dihydro-1,4,2-dioxazine (3ra)}

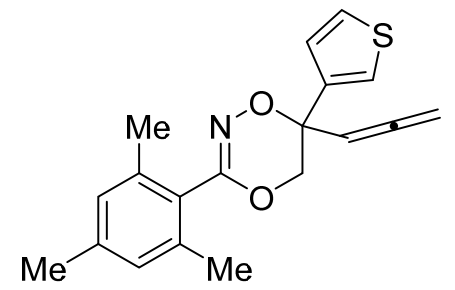

Prepared according to the general procedure (reaction time: $8 \mathrm{~h}$ ) as described above in $98 \%$ yield (31.9 mg). It was isolated by simple filtration and dried to afford a yellow liquid. ${ }^{1} \mathrm{H}$ NMR $\left(500 \mathrm{MHz}, \mathrm{CDCl}_{3}\right) \delta 7.4(\mathrm{dd}, J=3.0,1.3 \mathrm{~Hz}, 1 \mathrm{H}), 7.3(\mathrm{dd}, J=5.0,3.0 \mathrm{~Hz}, 1 \mathrm{H}), 7.1(\mathrm{dd}, J=$ 5.1, 1.3 Hz, 1H), $6.7(\mathrm{~s}, 2 \mathrm{H}), 5.4(\mathrm{t}, J=6.7 \mathrm{~Hz}, 1 \mathrm{H}), 5.0-4.9(\mathrm{~m}, 2 \mathrm{H}), 4.4(\mathrm{~d}, J=2.8 \mathrm{~Hz}, 2 \mathrm{H})$, $2.2(\mathrm{~s}, 3 \mathrm{H}), 2.1(\mathrm{~s}, 6 \mathrm{H}) . ;{ }^{13} \mathrm{C} \mathrm{NMR}\left(126 \mathrm{MHz}, \mathrm{CDCl}_{3}\right) \delta 206.9,152.8,139.6,138.4,136.4,127.2$, $126.7,125.3,125.0,122.2,92.1,78.3,72.8,67.7,20.1,18.1$; IR (thin film) $v_{\max } 2920,1956$, $1612,1455,1368,1308,1294,1170,1095,1034,983,947,902,883,851,789,740,647,580$, 528, 467, 460, 454, 441, 427, 420, $414 \mathrm{~cm}^{-1}$; HRMS (ESI) calculated for $\mathrm{C}_{19} \mathrm{H}_{20} \mathrm{O}_{2} \mathrm{NS}[\mathrm{M}+\mathrm{H}]^{+}$ 326.1209, found 326.1203. 


\section{3-(2,6-dichlorophenyl)-6-phenyl-6-(propa-1,2-dien-1-yl)-5,6-dihydro-1,4,2-dioxazine}

(3ab)<smiles>C=C=CC1(c2ccccc2)COC(c2c(Cl)cccc2Cl)=NO1</smiles>

Prepared according to the general procedure (reaction time: $5 \mathrm{~h}$ ) as described above in $81 \%$ yield $(27.9 \mathrm{mg})$. It was purified by flash chromatography (3\% EtOAc/PE) to afford a yellow solid (m.p. $\left.105-106{ }^{\circ} \mathrm{C}\right) .{ }^{1} \mathrm{H}$ NMR (400 MHz, Chloroform-d) $\delta 7.63-7.55$ (m, 2H), $7.48-$ $7.38(\mathrm{~m}, 2 \mathrm{H}), 7.41-7.34(\mathrm{~m}, 2 \mathrm{H}), 7.37-7.30(\mathrm{~m}, 3 \mathrm{H}), 7.30-7.25(\mathrm{~m}, 1 \mathrm{H}), 5.51(\mathrm{td}, J=6.7$, $1.0 \mathrm{~Hz}, 1 \mathrm{H}), 5.07-5.00(\mathrm{~m}, 4 \mathrm{H}), 5.03(\mathrm{~s}, 1 \mathrm{H}), 4.59(\mathrm{~d}, J=10.8 \mathrm{~Hz}, 1 \mathrm{H}), 4.50(\mathrm{dd}, J=10.8$, $1.0 \mathrm{~Hz}, 1 \mathrm{H}) ;{ }^{13} \mathrm{C}$ NMR $\left(126 \mathrm{MHz}, \mathrm{CDCl}_{3}\right) \delta 207.4,149.6,137.9,134.7,130.4,129.0,127.5$, 127.5, 126.9, 125.3, 91.6, 78.3, 75.4, 68.0.IR (thin film) $v_{\max } 3064,1956,1628,1579,1562$, $1448,1433,1369,1304,1261,1195,1139,1091,1060,966,902,855,791,763,731,698,461$, 445, 422, $\mathrm{cm}^{-1}$; HRMS (ESI) calculated for $\mathrm{C}_{18} \mathrm{H}_{14} \mathrm{Cl}_{2} \mathrm{NO}_{2}[\mathrm{M}+\mathrm{H}]^{+}$346.0396, found 346.0345.

\section{3-(2,6-dibromophenyl)-6-phenyl-6-(propa-1,2-dien-1-yl)-5,6-dihydro-1,4,2-dioxazine}

(3ac)<smiles>C=C=CC1(c2ccccc2)COC(c2c(Br)cccc2Br)=NO1</smiles>

Prepared according to the general procedure (reaction time: $7 \mathrm{~h}$ ) as described above in $68 \%$ yield $(29.6 \mathrm{mg})$. It was purified by flash chromatography $(3 \% \mathrm{EtOAc} / \mathrm{PE})$ to afford a white solid (m.p. $\left.114.0-114.4{ }^{\circ} \mathrm{C}\right) .{ }^{1} \mathrm{H} \mathrm{NMR}\left(400 \mathrm{MHz}, \mathrm{CDCl}_{3}\right) \delta 7.63-7.58(\mathrm{~m}, 2 \mathrm{H}), 7.55(\mathrm{~d}, J=$ $8.1 \mathrm{~Hz}, 2 \mathrm{H}), 7.46-7.34(\mathrm{~m}, 3 \mathrm{H}), 7.13(\mathrm{t}, J=8.1 \mathrm{~Hz}, 1 \mathrm{H}), 5.53(\mathrm{td}, J=6.7,1.0 \mathrm{~Hz}, 1 \mathrm{H}), 5.09$ $-4.99(\mathrm{~m}, 2 \mathrm{H}), 4.59(\mathrm{~d}, J=10.8 \mathrm{~Hz}, 1 \mathrm{H}), 4.51(\mathrm{dd}, J=10.8,1.0 \mathrm{~Hz}, 1 \mathrm{H}) ;{ }^{13} \mathrm{C}$ NMR $(126 \mathrm{MHz}$, $\left.\mathrm{CDCl}_{3}\right) \delta 207.4,151.6,138.0,133.1,132.6,131.0,130.8,130.7,127.6,127.5,125.4,123.5$, 92.0, 78.4, 75.4, 67.7; IR (thin film) $v_{\max } 3063,1956,1626,1576,1552,1491,1448,1425,1367$, 1303, 1193, 1129, 1058, 967, 937, 900, 854, 776, 763, 729, 698, 526, 457, 438, $424 \mathrm{~cm}^{-1}$; HRMS (ESI) calculated for $\mathrm{C}_{18} \mathrm{H}_{14} \mathrm{Br}_{2} \mathrm{NO}_{2}[\mathrm{M}+\mathrm{H}]^{+}$433.9386, found 433.9323 . 


\section{3-(2,6-dimethoxyphenyl)-6-phenyl-6-(propa-1,2-dien-1-yl)-5,6-dihydro-1,4,2-dioxazine}

(3ad)

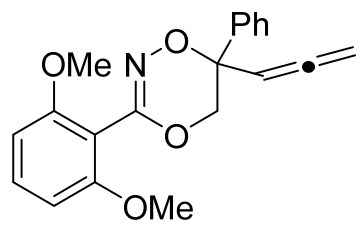

Prepared according to the general procedure (reaction time: $4 \mathrm{~h}$ ) as described above in $92 \%$ yield $(31.0 \mathrm{mg})$. It was purified by flash chromatography (3\% EtOAc/PE) to afford a yellow liquid. ${ }^{1} \mathrm{H}$ NMR $\left(500 \mathrm{MHz}, \mathrm{CDCl}_{3}\right) \delta 7.55-7.49(\mathrm{~m}, 2 \mathrm{H}), 7.37-7.32(\mathrm{~m}, 2 \mathrm{H}), 7.30-7.25(\mathrm{~m}$, 1H), $7.21(\mathrm{t}, J=8.5 \mathrm{~Hz}, 1 \mathrm{H}), 6.44(\mathrm{~d}, J=8.4 \mathrm{~Hz}, 2 \mathrm{H}), 5.43(\mathrm{td}, J=6.7,0.8 \mathrm{~Hz}, 1 \mathrm{H}), 4.91(\mathrm{~d}, J$ $=6.7 \mathrm{~Hz}, 2 \mathrm{H}), 4.47(\mathrm{~d}, J=10.7 \mathrm{~Hz}, 1 \mathrm{H}), 4.43(\mathrm{dd}, J=10.7,0.8 \mathrm{~Hz}, 1 \mathrm{H}), 3.67(\mathrm{~s}, 6 \mathrm{H}) ;{ }^{13} \mathrm{C} \mathrm{NMR}$ $\left(126 \mathrm{MHz}, \mathrm{CDCl}_{3}\right) \delta 208.4,161.1,159.2,150.6,143.4,139.5,136.7,131.7,128.3,128.1,126.4$, $125.8,109.6,106.3,103.8,102.8,92.6,78.8,75.8,69.5,56.0,55.5$. HRMS (ESI) calculated for $\mathrm{C}_{20} \mathrm{H}_{20} \mathrm{NO}_{4}[\mathrm{M}+\mathrm{H}]^{+} 338.1387$, found 338.1382 .

\section{6-phenyl-6-(propa-1,2-dien-1-yl)-3-(2,4,5-trimethylphenyl)-5,6-dihydro-1,4,2-dioxazine} (3ae)<smiles>C=C=CC1(c2ccccc2)COC(c2cc(C)c(C)cc2C)=NO1</smiles>

Prepared according to the general procedure (reaction time: $17 \mathrm{~h}$ ) as described above in $67 \%$ yield $(21.4 \mathrm{mg})$. It was purified by flash chromatography (3\% EtOAc/PE) to afford a yellow liquid. ${ }^{1} \mathrm{H}$ NMR $\left(400 \mathrm{MHz}, \mathrm{CDCl}_{3}\right) \delta 7.62-7.54(\mathrm{~m}, 2 \mathrm{H}), 7.46-7.38(\mathrm{~m}, 2 \mathrm{H}), 7.40-7.33(\mathrm{~m}$, $1 \mathrm{H}), 7.25(\mathrm{~s}, 1 \mathrm{H}), 6.97(\mathrm{~s}, 1 \mathrm{H}), 5.45(\mathrm{td}, J=6.7,1.0 \mathrm{~Hz}, 1 \mathrm{H}), 5.08-4.94(\mathrm{~m}, 2 \mathrm{H}), 4.57(\mathrm{~d}, J=$ $10.7 \mathrm{~Hz}, 1 \mathrm{H}), 4.49(\mathrm{dd}, J=10.8,1.0 \mathrm{~Hz}, 1 \mathrm{H}), 2.31(\mathrm{~s}, 3 \mathrm{H}), 2.23(\mathrm{~s}, 6 \mathrm{H}), 2.23(\mathrm{~s}, 3 \mathrm{H}) ;{ }^{13} \mathrm{C} \mathrm{NMR}$ $\left(126 \mathrm{MHz}, \mathrm{CDCl}_{3}\right) \delta 207.1,154.2,138.1,137.6,133.4,132.7,131.2,128.8,127.5,127.3,126.4$, 125.1, 91.5, 78.0, 74.5, 68.3, 18.9, 18.5, 18.1.IR (thin film) $v_{\max } 2922,1955,1764,1604,1492$, $1448,1403,1316,1276,1239,1148,1085,1062,967,942,855,763,699,457,437,424 \mathrm{~cm}^{-1}$; HRMS (ESI) calculated for $\mathrm{C}_{21} \mathrm{H}_{22} \mathrm{NO}_{2}[\mathrm{M}+\mathrm{H}]^{+} 320.1645$, found 320.1579 . 
6-phenyl-6-(propa-1,2-dien-1-yl)-3-(p-tolyl)-5,6-dihydro-1,4,2-dioxazine (3af)

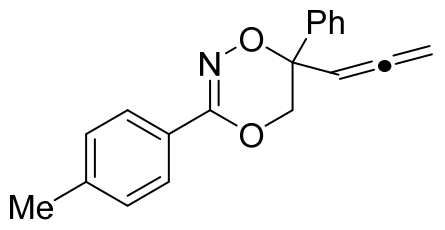

Prepared according to the general procedure (reaction time: $5 \mathrm{~h}$ ) as described above in $41 \%$ yield $(11.9 \mathrm{mg})$. It was purified by flash chromatography $(3 \%$ EtOAc/PE) to afford a yellow solid (m.p. $\left.101-102{ }^{\circ} \mathrm{C}\right) .{ }^{1} \mathrm{H}$ NMR $\left(400 \mathrm{MHz}, \mathrm{CDCl}_{3}\right) \delta 7.76-7.68(\mathrm{~m}, 2 \mathrm{H}), 7.59-7.52(\mathrm{~m}$, 2H), $7.47-7.37(\mathrm{~m}, 3 \mathrm{H}), 7.39-7.30(\mathrm{~m}, 1 \mathrm{H}), 7.23-7.16(\mathrm{~m}, 2 \mathrm{H}), 5.40(\mathrm{td}, J=6.7,1.2 \mathrm{~Hz}$, $1 \mathrm{H}), 5.01(\mathrm{dd}, J=11.5,6.7 \mathrm{~Hz}, 1 \mathrm{H}), 4.94(\mathrm{dd}, J=11.5,6.7 \mathrm{~Hz}, 1 \mathrm{H}), 4.59(\mathrm{~d}, J=10.8 \mathrm{~Hz}, 1 \mathrm{H})$, $4.43(\mathrm{dd}, J=10.7,1.2 \mathrm{~Hz}, 1 \mathrm{H}), 2.38$ (s, 3H); ${ }^{13} \mathrm{C} \mathrm{NMR}\left(126 \mathrm{MHz}, \mathrm{CDCl}_{3}\right) \delta$ 207.1, 152.0, 139.6, 138.1, 128.7, 128.6, 127.9, 127.5, 127.4, 127.1, 126.3, 125.0, 124.7, 91.0, 77.9, 74.9, 68.3, 20.4. IR (thin film) $v_{\max } 2922,1955,1762,1611,1568,1516,1493,1448,1378,1329,1182,1114$, 1021, 972, 938, 906, 854, 824, 761, 718, 700, 661, 496, 446, 438, $430 \mathrm{~cm}^{-1}$; HRMS (ESI) calculated for $\mathrm{C}_{19} \mathrm{H}_{18} \mathrm{NO}_{2}[\mathrm{M}+\mathrm{H}]^{+}$292.1332, found 292.1330.

\section{3-(naphthalen-1-yl)-6-phenyl-6-(propa-1,2-dien-1-yl)-5,6-dihydro-1,4,2-dioxazine (3ag)}<smiles>C=CCC1(c2ccccc2)COC(c2ccc3ccccc3c2)=NO1</smiles>

Prepared according to the general procedure (reaction time: $74 \mathrm{~h}$ ) as described above in $28 \%$ yield $(9.0 \mathrm{mg})$. It was purified by flash chromatography $(3 \%$ EtOAc/PE) to afford a yellow solid (m.p. $\left.107-108^{\circ} \mathrm{C}\right) .{ }^{1} \mathrm{H}$ NMR $\left(500 \mathrm{MHz}, \mathrm{CDCl}_{3}\right) \delta 8.22-8.19(\mathrm{~m}, 1 \mathrm{H}), 7.92-7.86(\mathrm{~m}$, 1H), $7.85-7.71(\mathrm{~m}, 5 \mathrm{H}), 7.53-7.48(\mathrm{~m}, 3 \mathrm{H}), 7.46-7.41(\mathrm{~m}, 2 \mathrm{H}), 7.38-7.31(\mathrm{~m}, 2 \mathrm{H}), 7.31$ $-7.25(\mathrm{~m}, 1 \mathrm{H}), 5.35(\mathrm{t}, J=6.7 \mathrm{~Hz}, 1 \mathrm{H}), 4.95(\mathrm{dd}, J=11.6,6.7 \mathrm{~Hz}, 1 \mathrm{H}), 4.87$ (dd, $J=11.6,6.7$ $\mathrm{Hz}, 1 \mathrm{H}), 4.58(\mathrm{~d}, J=10.7 \mathrm{~Hz}, 1 \mathrm{H}), 4.43(\mathrm{~d}, J=10.7 \mathrm{~Hz}, 1 \mathrm{H}) ;{ }^{13} \mathrm{C} \mathrm{NMR}\left(126 \mathrm{MHz}, \mathrm{CDCl}_{3}\right) \delta$ $207.2,151.9,138.0,133.3,131.7,127.7,127.6,127.5,127.0,126.7,126.1,125.4,125.0,124.7$, 121.8, 91.0, 78.0, 75.2, 68.5; IR (thin film) $v_{\max } 2923,1609,1448,1317,1275,1261,1236$, 1199, 1133, 1105, 975, 909, 861, 821, 751, 700, 525, 476, 458, 446, 437, 424, $407 \mathrm{~cm}^{-1}$; HRMS (ESI) calculated for $\mathrm{C}_{22} \mathrm{H}_{18} \mathrm{NO}_{2}[\mathrm{M}+\mathrm{H}]^{+} 328.1332$, found 328.1330 . 
(E)-3-(3-mesityl-6-phenyl-5,6-dihydro-1,4,2-dioxazin-6-yl)allyl benzoate (4)

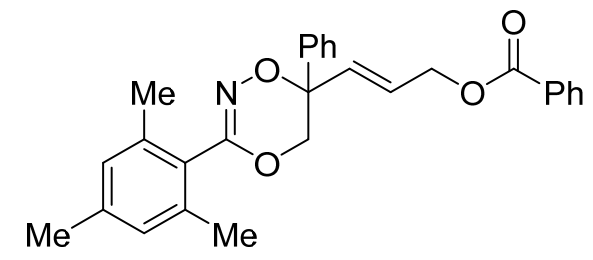

Prepared according to the general procedure (reaction time: $25 \mathrm{~h}$ ) as described above in $40 \%$ yield $(8.6 \mathrm{mg})$. It was purified by flash chromatography $(10 \%$ EtOAc/PE) to afford a white liquid. ${ }^{1} \mathrm{H}$ NMR $\left(500 \mathrm{MHz}, \mathrm{CDCl}_{3}\right) \delta 8.1-8.0(\mathrm{~m}, 2 \mathrm{H}), 7.6$ - $7.5(\mathrm{~m}, 3 \mathrm{H}), 7.5-7.4(\mathrm{~m}, 4 \mathrm{H})$, $7.4-7.3(\mathrm{~m}, 1 \mathrm{H}), 6.8(\mathrm{~s}, 2 \mathrm{H}), 6.1-6.0(\mathrm{~m}, 2 \mathrm{H}), 4.9-4.8(\mathrm{~m}, 2 \mathrm{H}), 4.7(\mathrm{~d}, J=11.0 \mathrm{~Hz}, 1 \mathrm{H})$ $4.5(\mathrm{~d}, J=11.0 \mathrm{~Hz}, 1 \mathrm{H}), 2.2(\mathrm{~s}, 3 \mathrm{H}), 2.0(\mathrm{~s}, 6 \mathrm{H}) ;{ }^{13} \mathrm{C} \mathrm{NMR}\left(126 \mathrm{MHz}, \mathrm{CDCl}_{3}\right) \delta 153.8,139.5$, 138.6, 137.4, 133.1, 132.1, 129.7, 128.8, 128.7, 128.4, 128.2, 126.7, 77.2, 68.7, 64.2, 21.1, 19.2, 19.2; HRMS (ESI) calculated for $\mathrm{C}_{28} \mathrm{H}_{28} \mathrm{NO}_{4}[\mathrm{M}+\mathrm{H}]^{+}$442.2013, found 442.2014 .

\section{3-mesityl-6-phenyl-6-propyl-5,6-dihydro-1,4,2-dioxazine (5)}

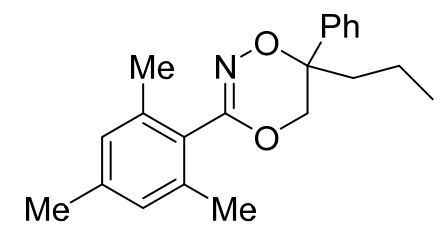

Prepared according to the general procedure as described above in $85 \%$ yield (13.8 $\mathrm{mg})$. It was purified by flash chromatography (10\% EtOAc/PE) to afford a white liquid. liquid. ${ }^{1} \mathrm{H}$ NMR $\left(500 \mathrm{MHz}, \mathrm{CDCl}_{3}\right) \delta 7.5-7.4(\mathrm{~m}, 2 \mathrm{H}), 7.3-7.3(\mathrm{~m}, 2 \mathrm{H}), 7.3-7.2(\mathrm{~m}, 1 \mathrm{H}), 6.7(\mathrm{~s}, 2 \mathrm{H}), 4.7(\mathrm{~d}$ $J=10.8 \mathrm{~Hz}, 1 \mathrm{H}), 4.3(\mathrm{~d}, J=10.8 \mathrm{~Hz}, 1 \mathrm{H}), 2.1(\mathrm{~s}, 3 \mathrm{H}), 1.8(\mathrm{~s}, 6 \mathrm{H}), 1.4-1.3(\mathrm{~m}, 2 \mathrm{H}), 1.2-1.2$ (m, 2H), $0.8-0.8(\mathrm{~m}, 3 \mathrm{H}) ;{ }^{13} \mathrm{C} \mathrm{NMR}\left(126 \mathrm{MHz}, \mathrm{CDCl}_{3}\right) \delta 153.1,138.6,138.2,136.4,127.4$ $127.1,126.7,126.7,125.6,68.3,39.6,20.1,17.9,15.0,13.3,13.1$; IR (thin film) $v_{\max } 2960$ $2925,1612,1447,1377,1308,1295,1171,1085,1031,995,944,905,850,767,701,593,536$ 498, 458, 443, 433, 420, 415 $\mathrm{cm}^{-1}$; HRMS (ESI) calculated for $\mathrm{C}_{21} \mathrm{H}_{26} \mathrm{NO}_{2}[\mathrm{M}+\mathrm{H}]^{+} 324.1958$, found 324.1955 . 
${ }^{1} \mathrm{H}$ and ${ }^{13} \mathrm{C}$ NMR Spectra of New Substrates

${ }^{1} \mathrm{H}$ NMR spectrum $\left(500 \mathrm{MHz}, \mathrm{CDCl}_{3}\right)$

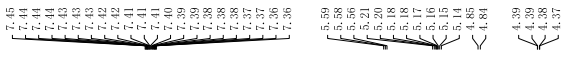
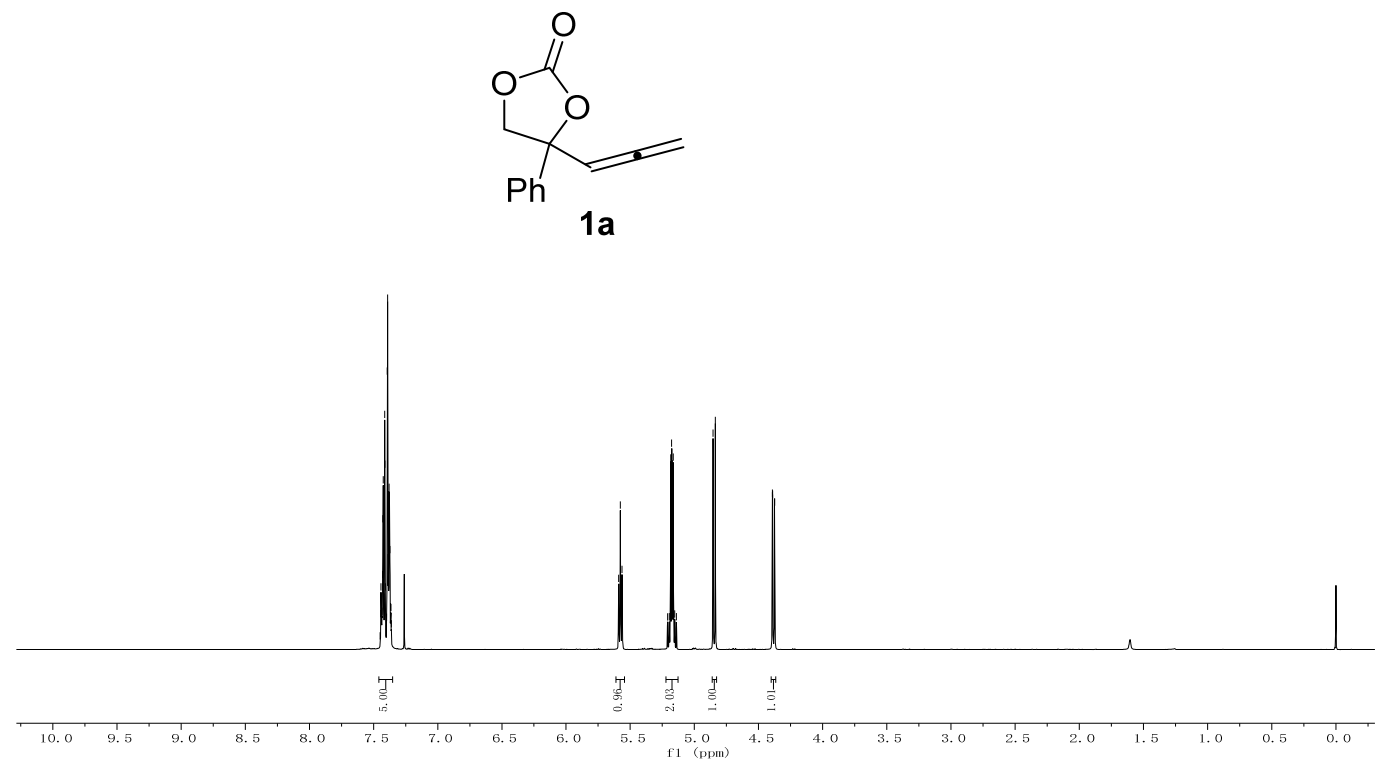

${ }^{13} \mathrm{C} \mathrm{NMR}$ spectrum $\left(126 \mathrm{MHz}, \mathrm{CDCl}_{3}\right)$

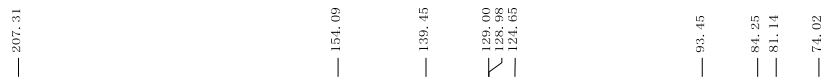

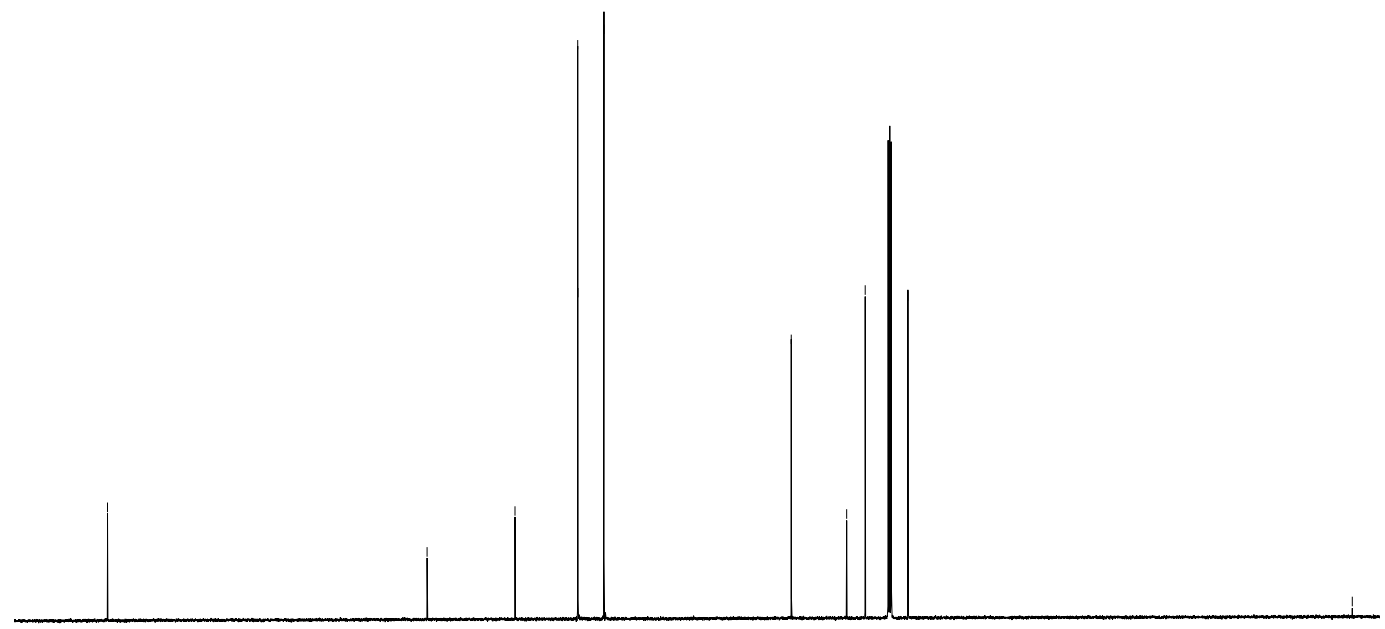

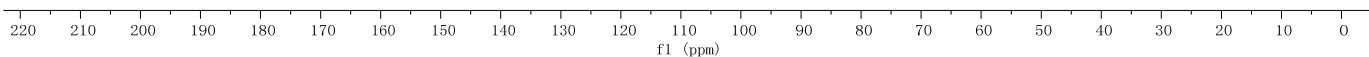


${ }^{1} \mathrm{H}$ NMR spectrum $\left(400 \mathrm{MHz}, \mathrm{CDCl}_{3}\right)$

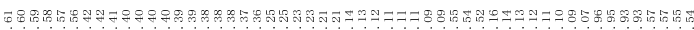

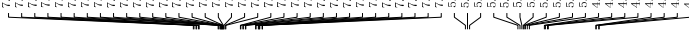<smiles>C=CC1(c2ccccc2F)COC(=O)O1</smiles>

$1 b$

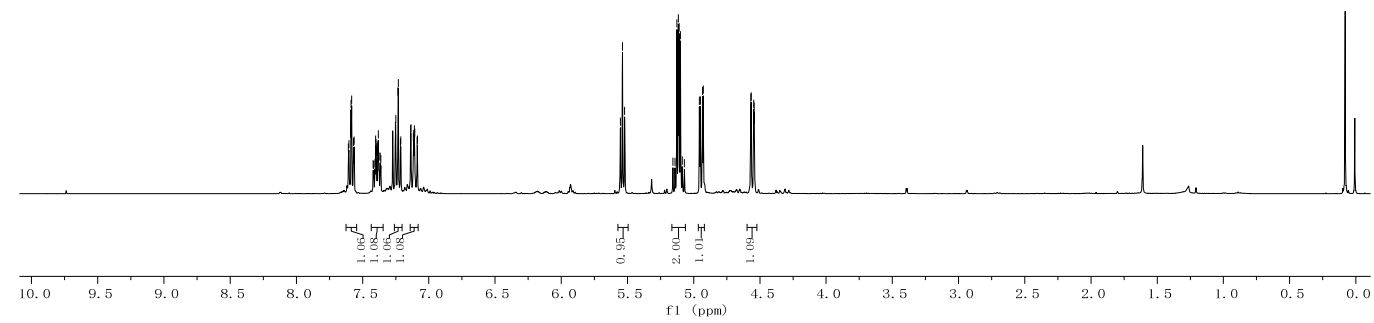

${ }^{13} \mathrm{C}$ NMR spectrum $\left(101 \mathrm{MHz}, \mathrm{CDCl}_{3}\right)$

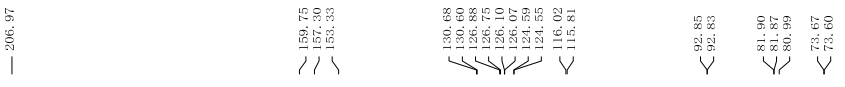
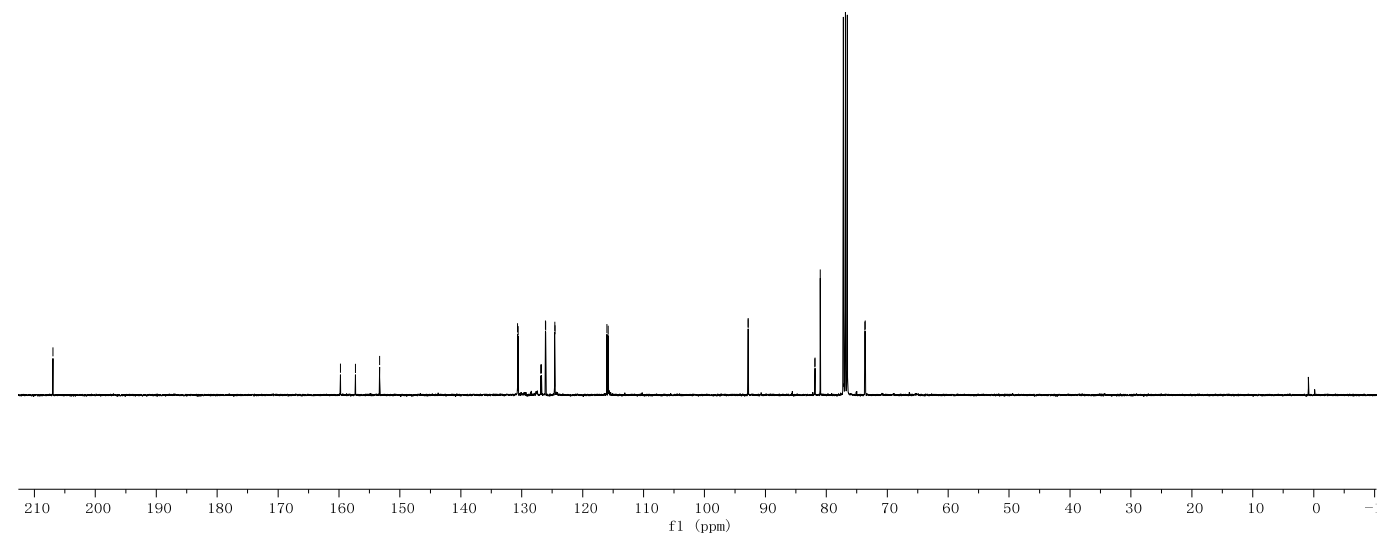
${ }^{1} \mathrm{H}$ NMR spectrum $\left(400 \mathrm{MHz}, \mathrm{CDCl}_{3}\right)$

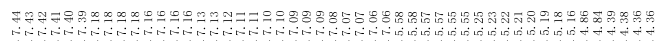<smiles>C=CCC1(c2cccc(F)c2)COC(=O)O1</smiles>

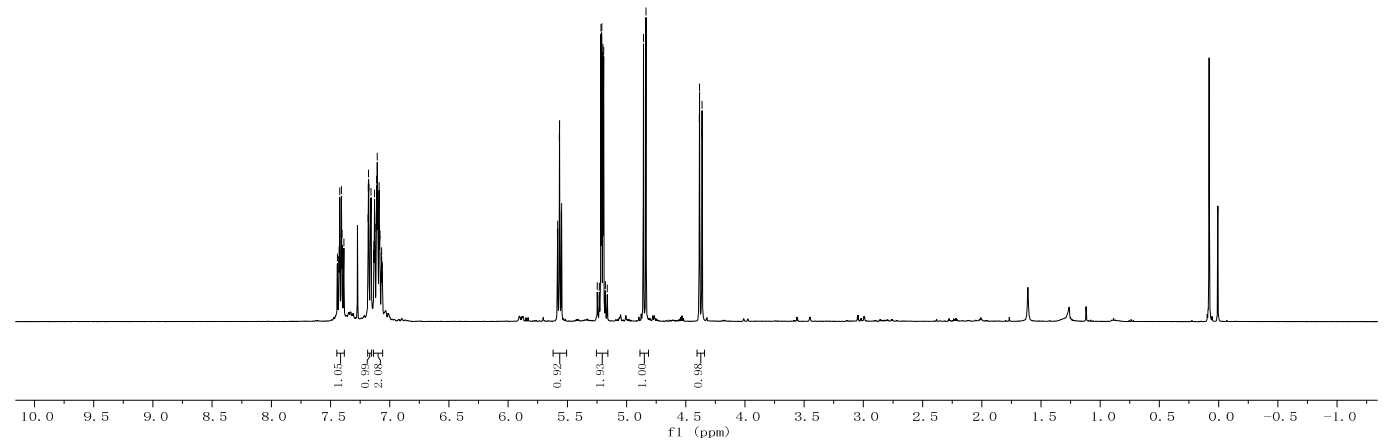

${ }^{13} \mathrm{C}$ NMR spectrum $\left(101 \mathrm{MHz}, \mathrm{CDCl}_{3}\right)$

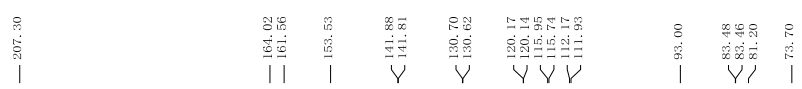
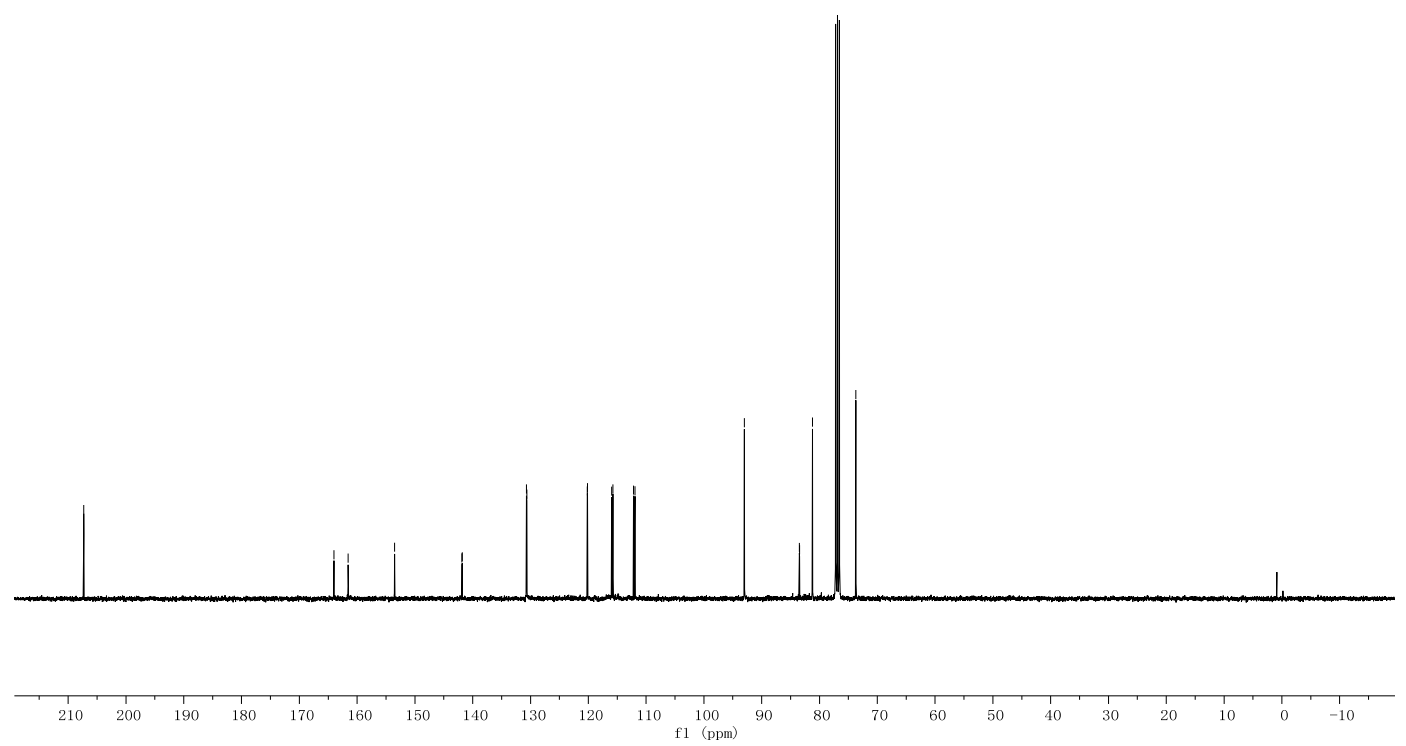
${ }^{1} \mathrm{H}$ NMR spectrum $\left(400 \mathrm{MHz}, \mathrm{CDCl}_{3}\right)$
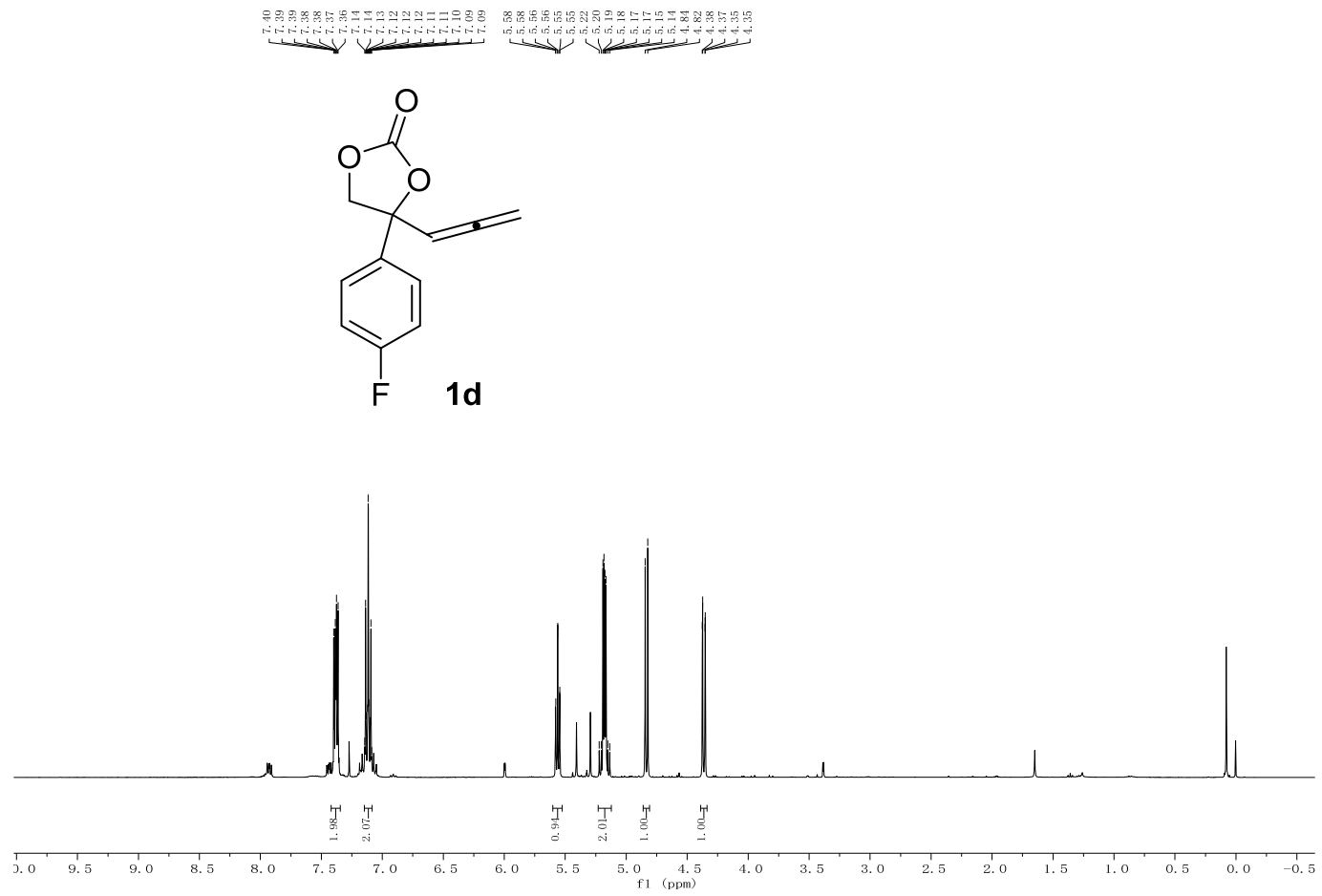

${ }^{13} \mathrm{C}$ NMR spectrum $\left(101 \mathrm{MHz}, \mathrm{CDCl}_{3}\right)$

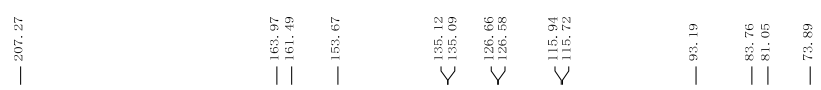
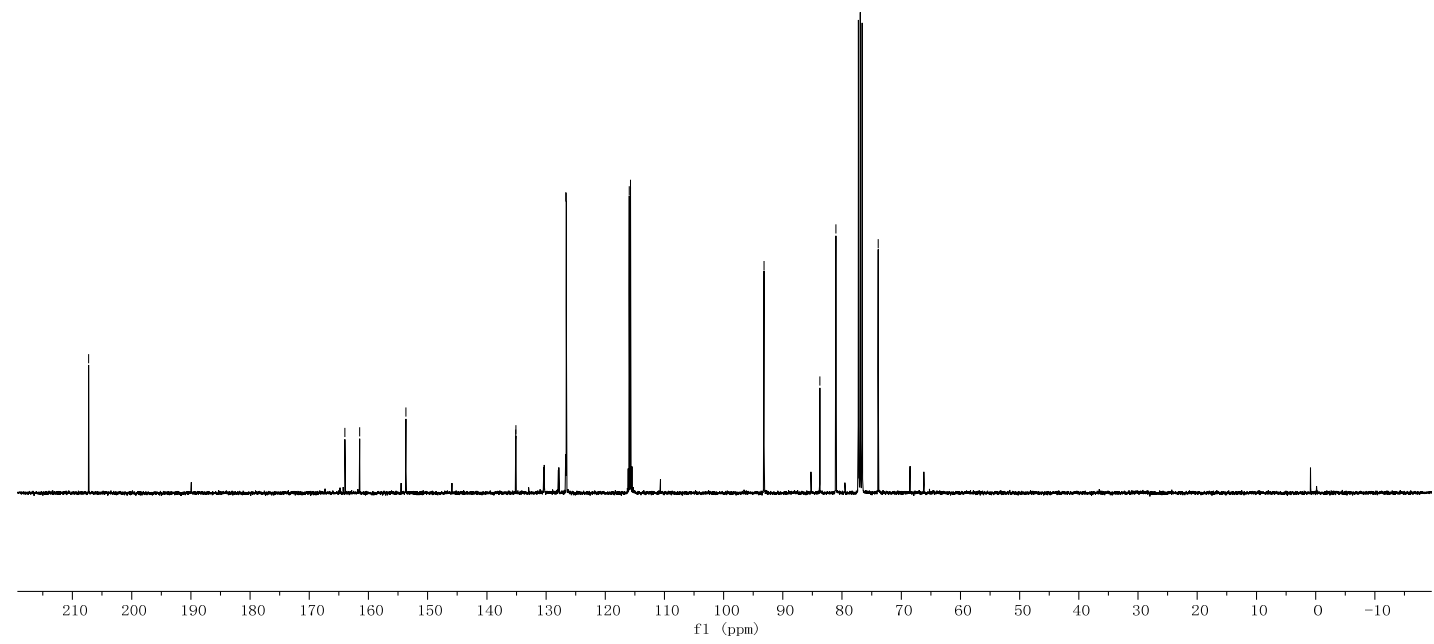
${ }^{1} \mathrm{H}$ NMR spectrum $\left(500 \mathrm{MHz}, \mathrm{CDCl}_{3}\right)$
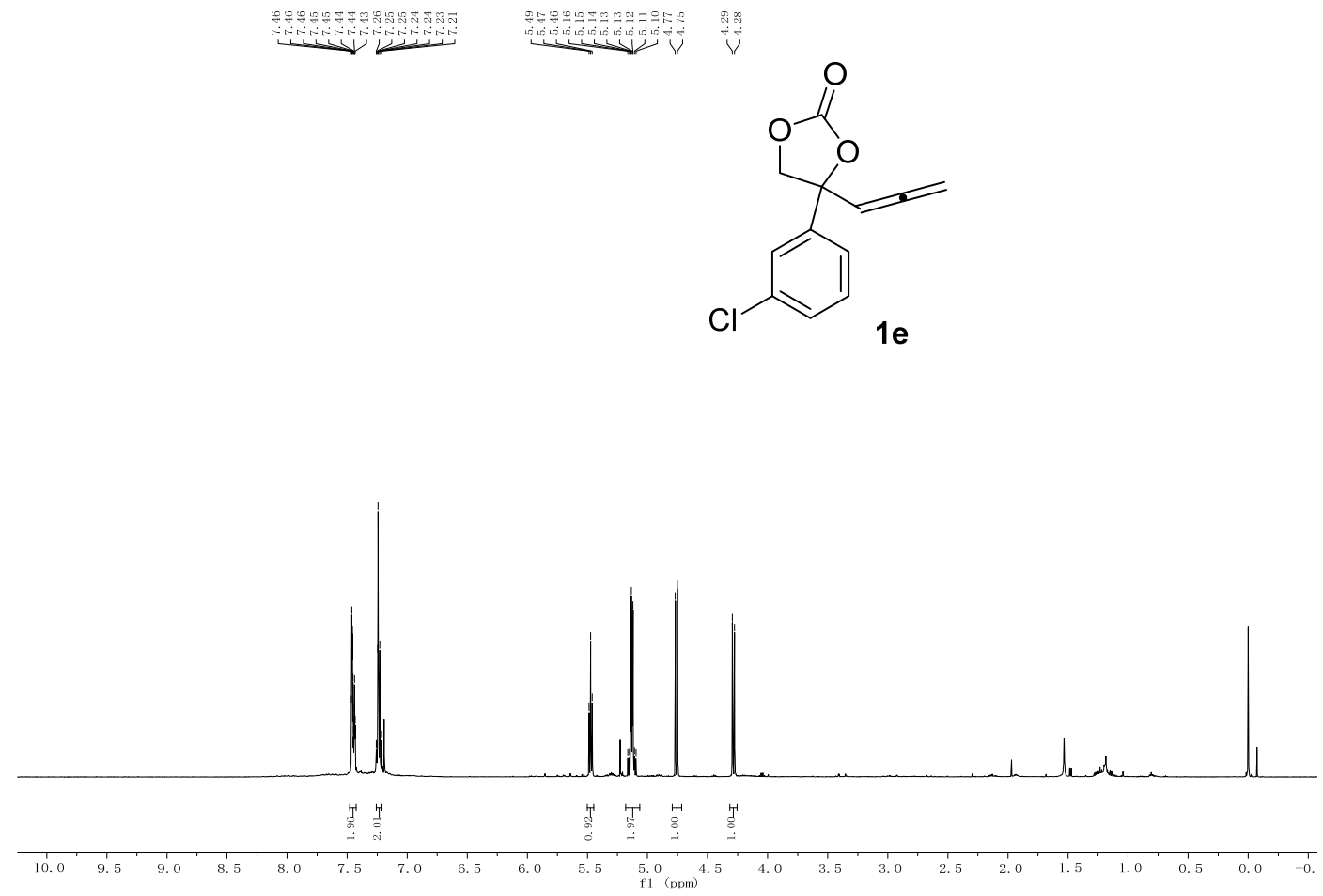

${ }^{13} \mathrm{C}$ NMR spectrum $\left(126 \mathrm{MHz}, \mathrm{CDCl}_{3}\right)$

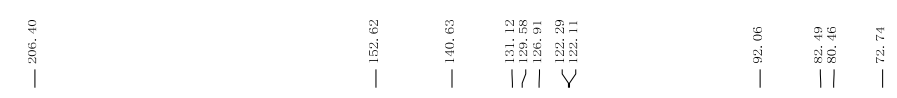
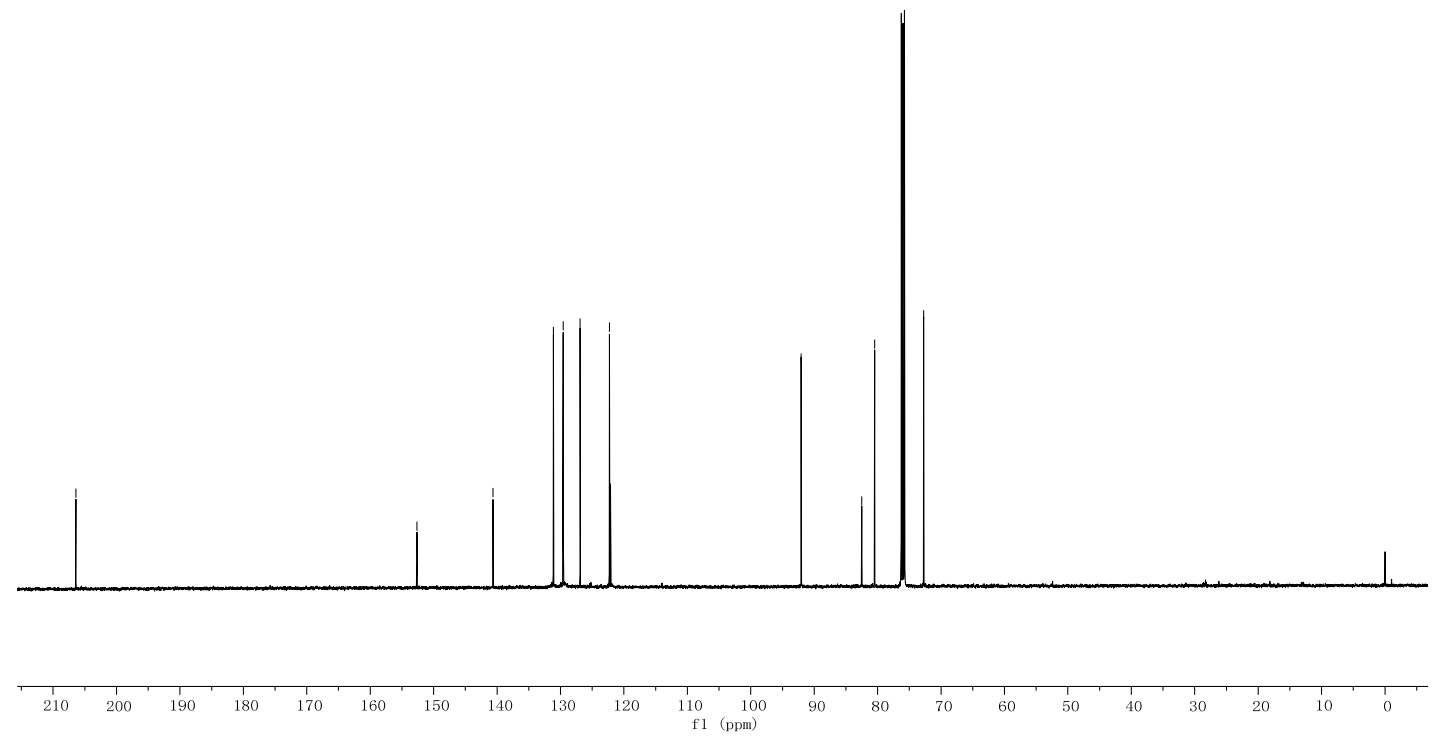
${ }^{1} \mathrm{H}$ NMR spectrum $\left(500 \mathrm{MHz}, \mathrm{CDCl}_{3}\right)$
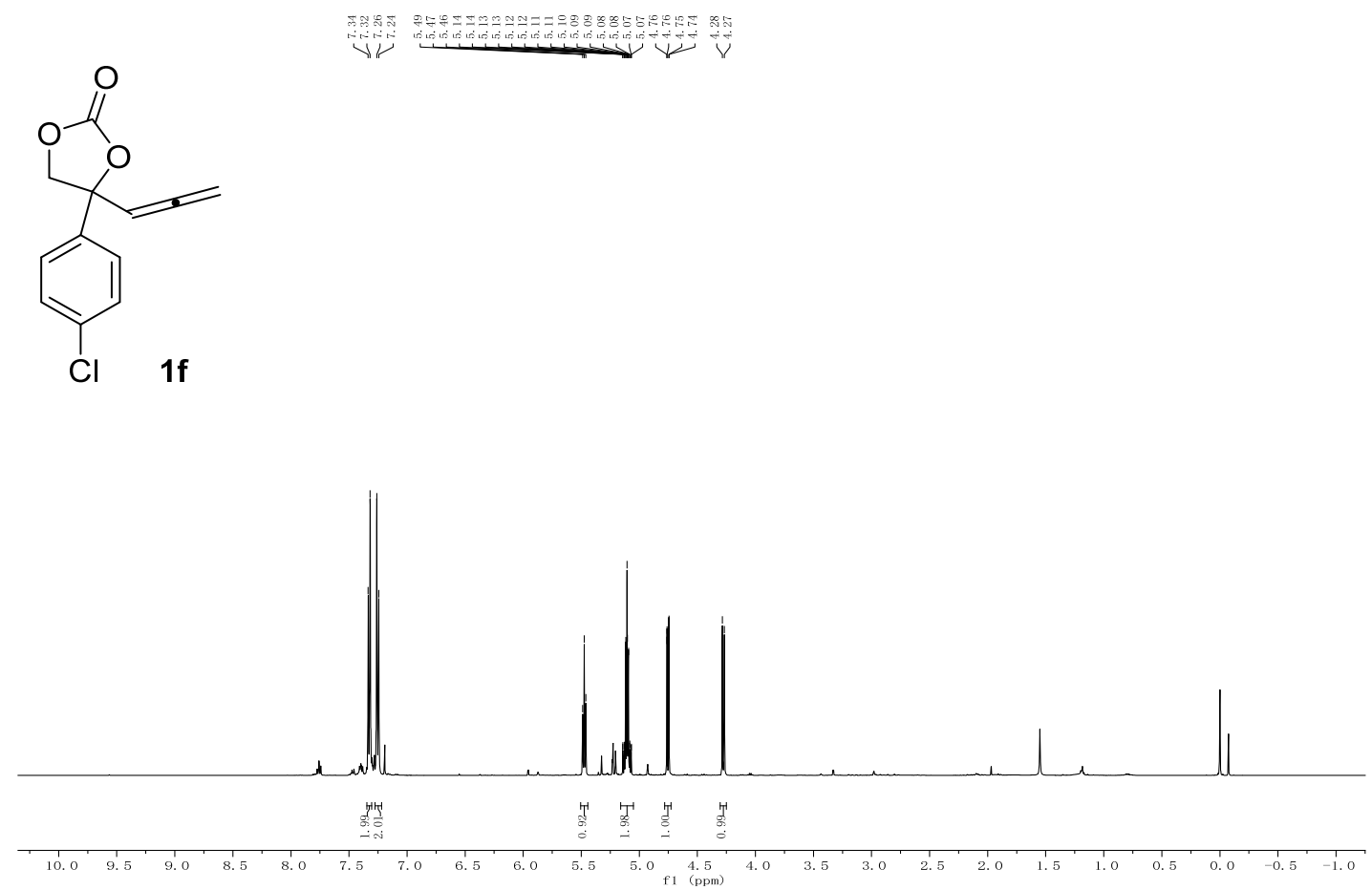

${ }^{13} \mathrm{C}$ NMR spectrum $\left(126 \mathrm{MHz}, \mathrm{CDCl}_{3}\right)$
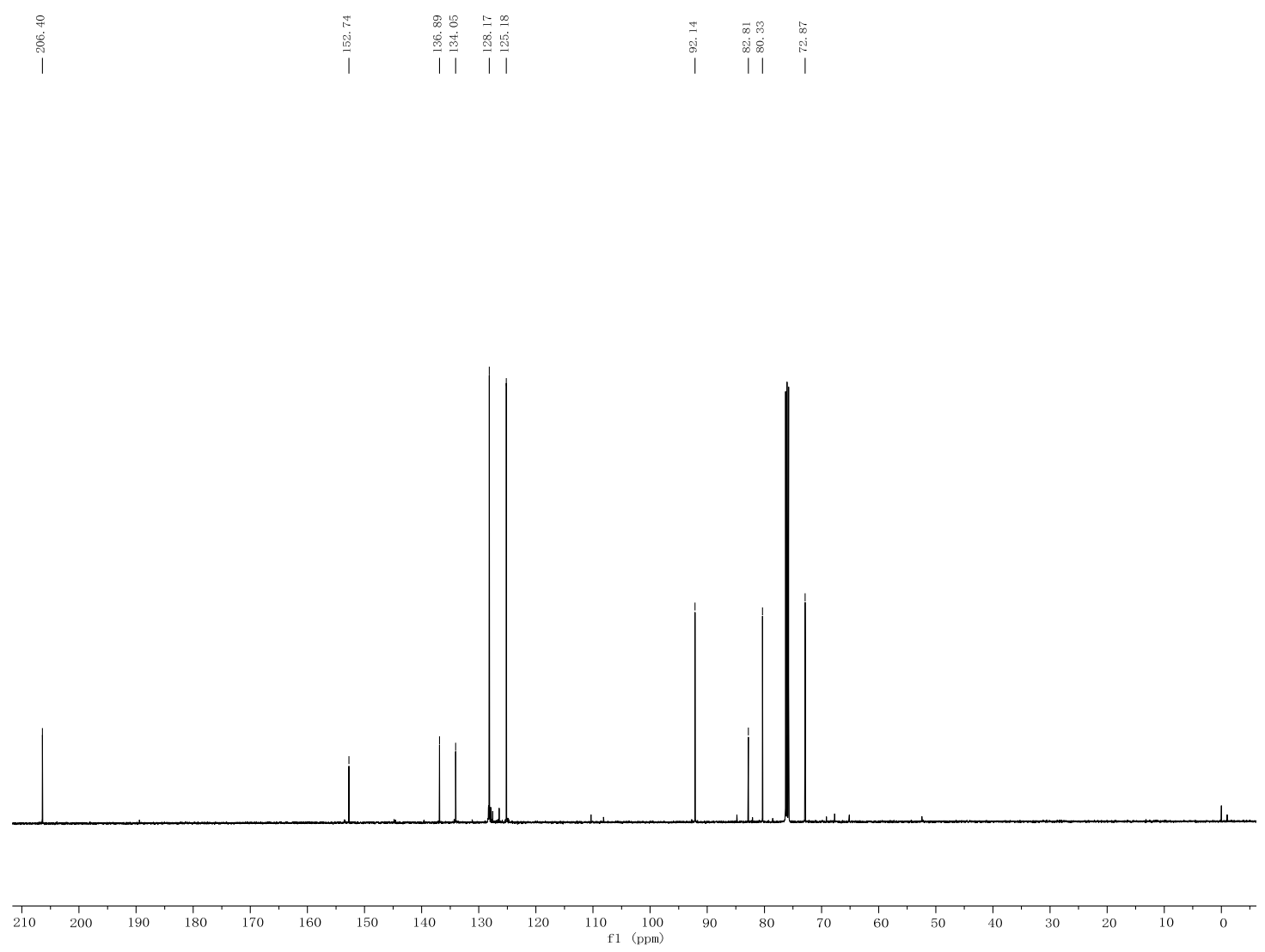
${ }^{1} \mathrm{H}$ NMR spectrum (500 MHz, $\mathrm{CDCl}_{3}$ )

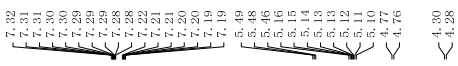<smiles>C=CC1(c2cccc(Br)c2)COC(=O)O1</smiles>

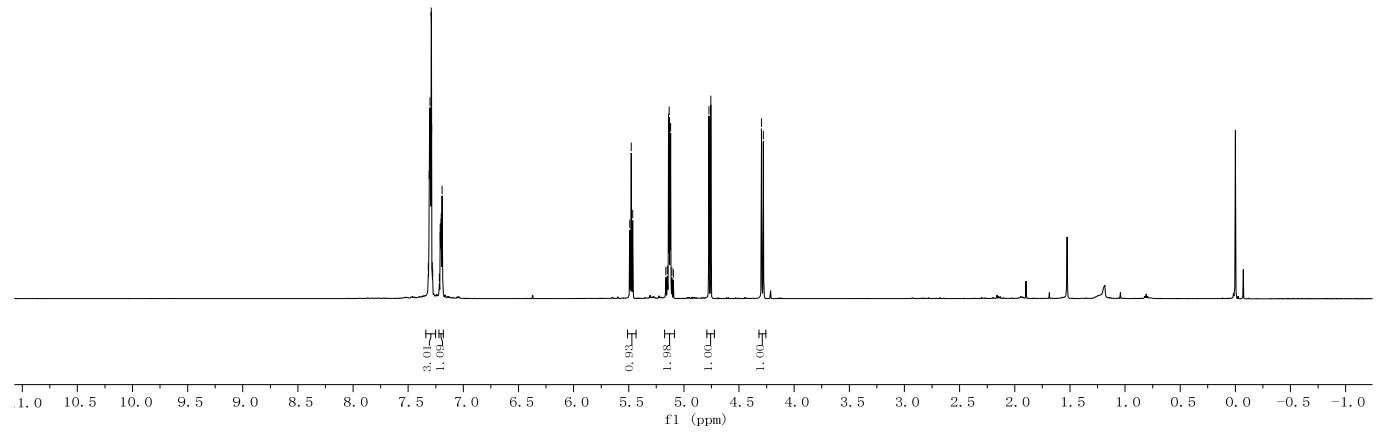

${ }^{13} \mathrm{C}$ NMR spectrum $\left(126 \mathrm{MHz}, \mathrm{CDCl}_{3}\right)$

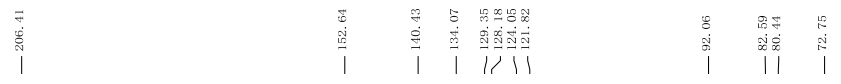
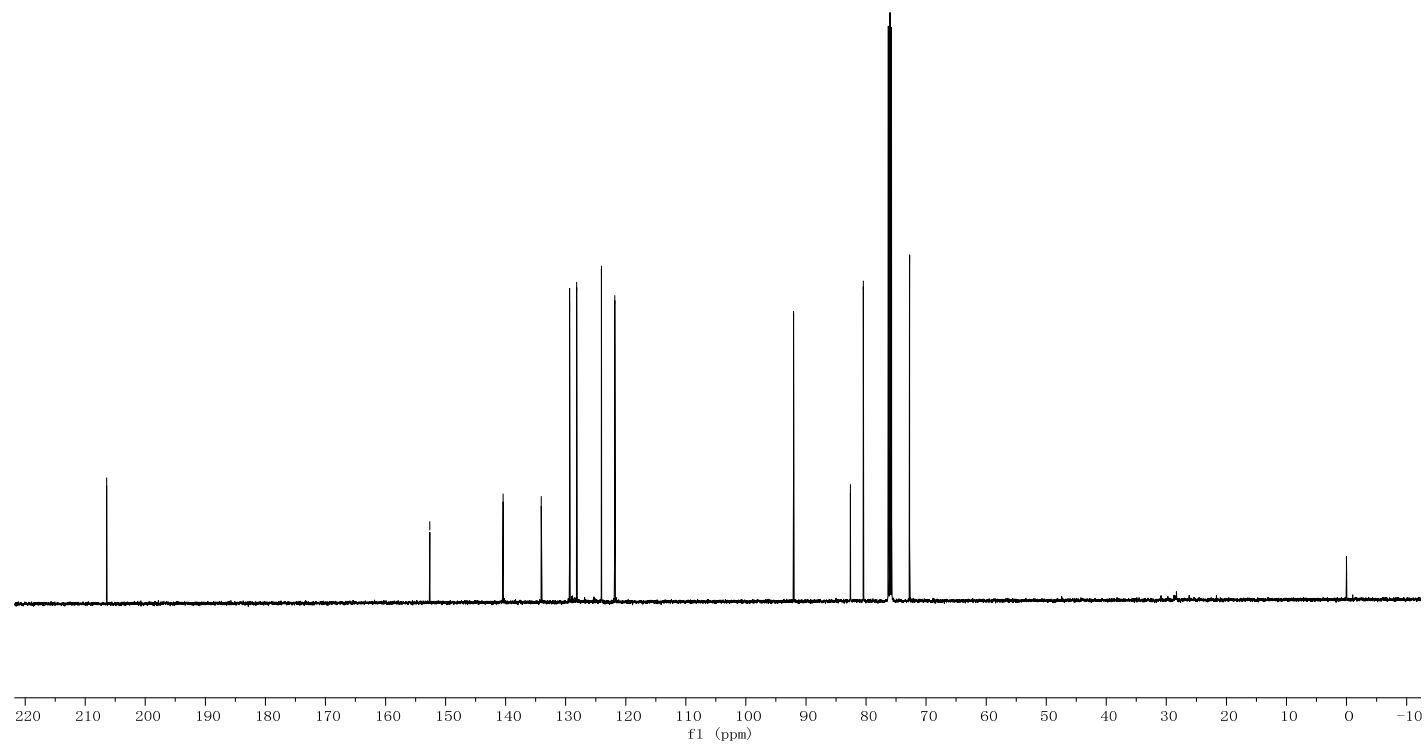
${ }^{1} \mathrm{H}$ NMR spectrum $\left(500 \mathrm{MHz}, \mathrm{CDCl}_{3}\right)$
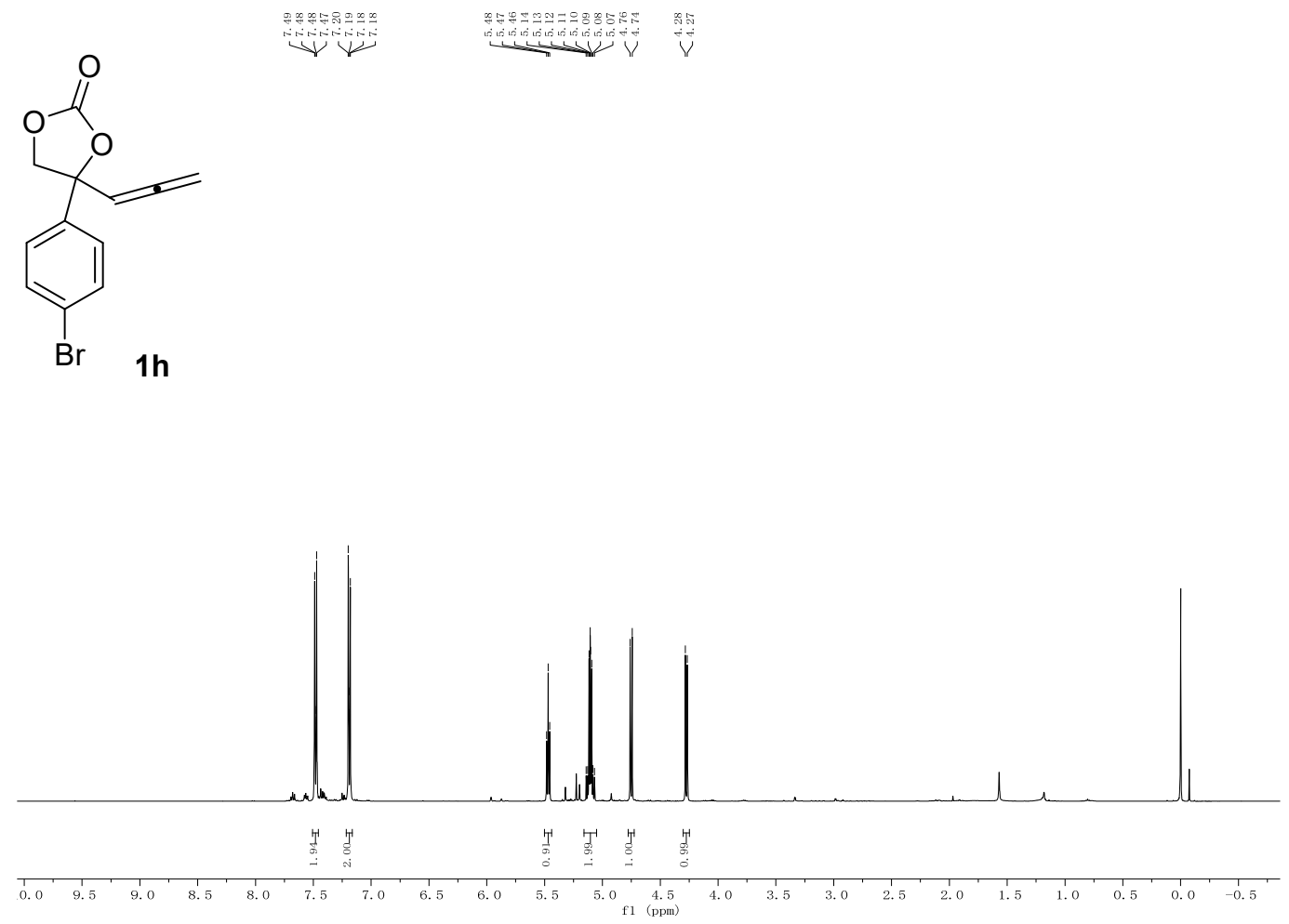

${ }^{13} \mathrm{C}$ NMR spectrum (126 MHz, $\left.\mathrm{CDCl}_{3}\right)$

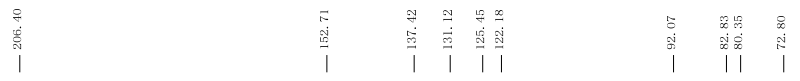
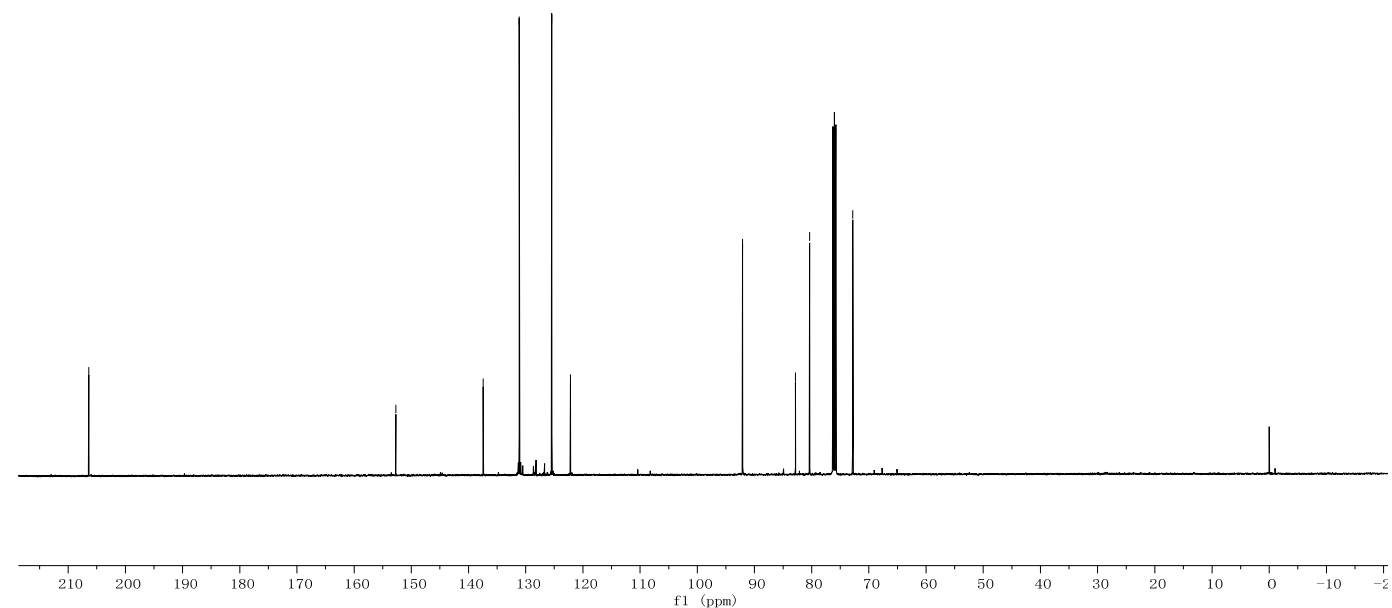
${ }^{1} \mathrm{H}$ NMR spectrum $\left(500 \mathrm{MHz}, \mathrm{CDCl}_{3}\right)$

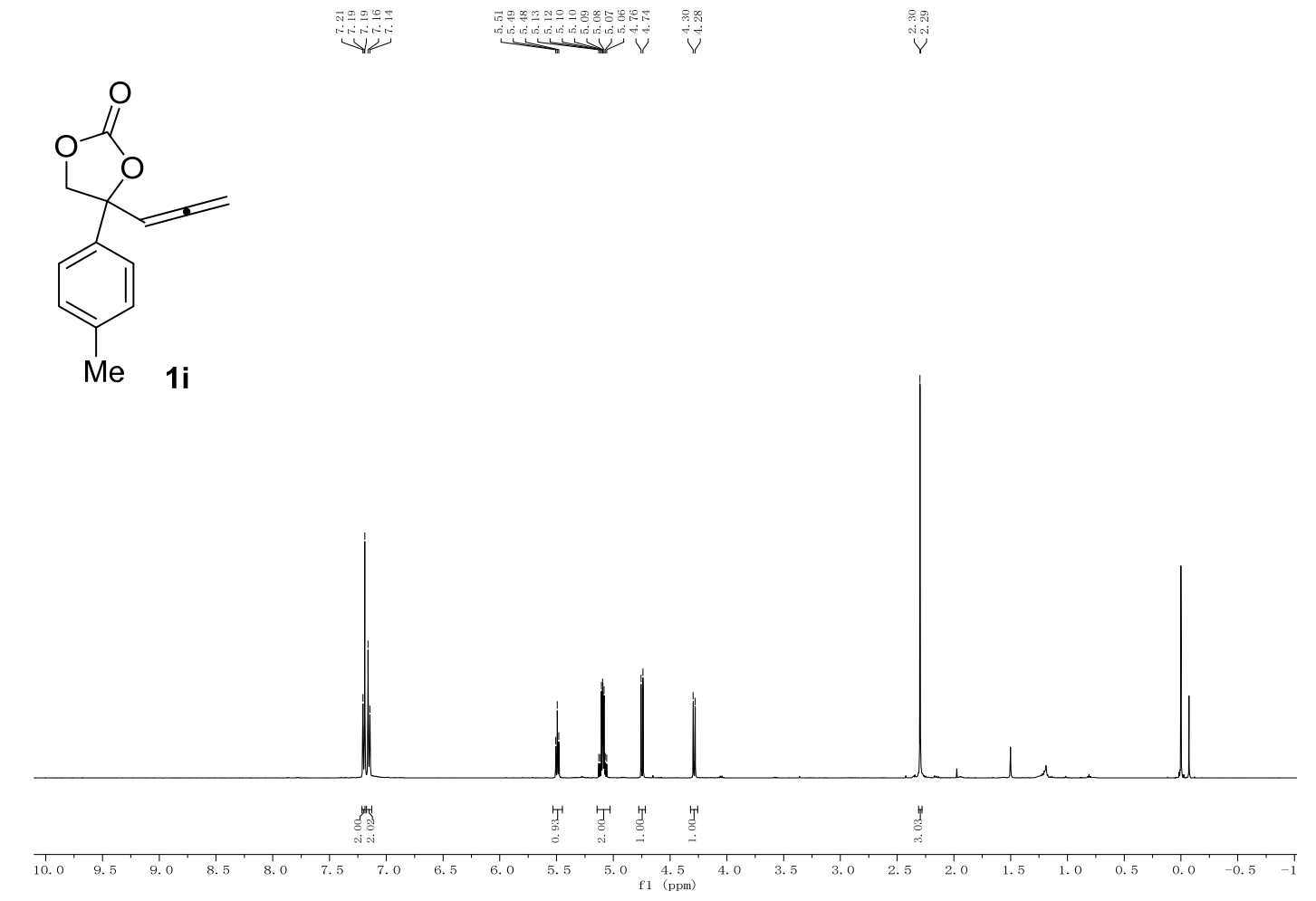

${ }^{13} \mathrm{C}$ NMR spectrum $\left(126 \mathrm{MHz}, \mathrm{CDCl}_{3}\right)$

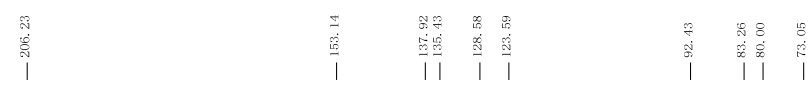
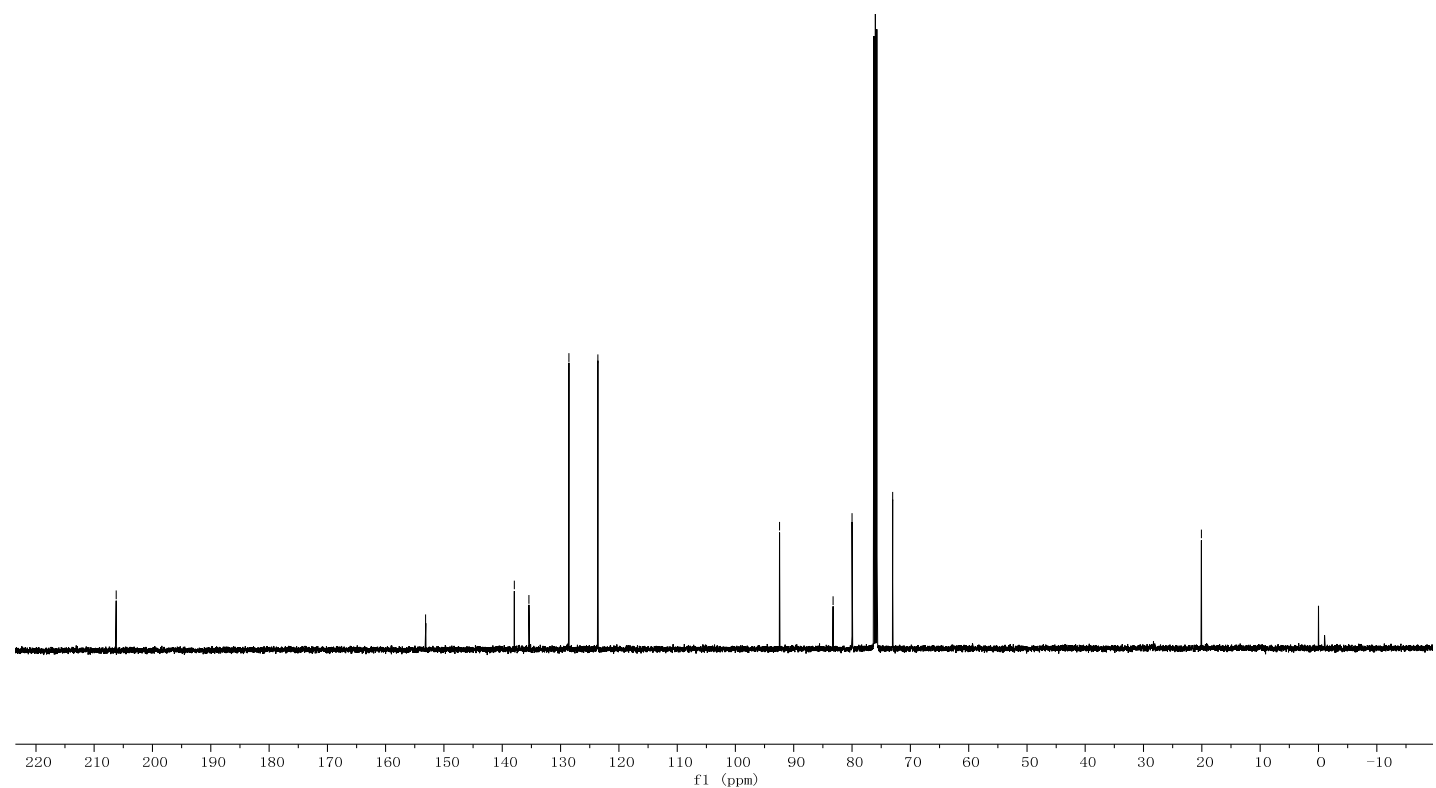
${ }^{1} \mathrm{H}$ NMR spectrum $\left(500 \mathrm{MHz}, \mathrm{CDCl}_{3}\right)$

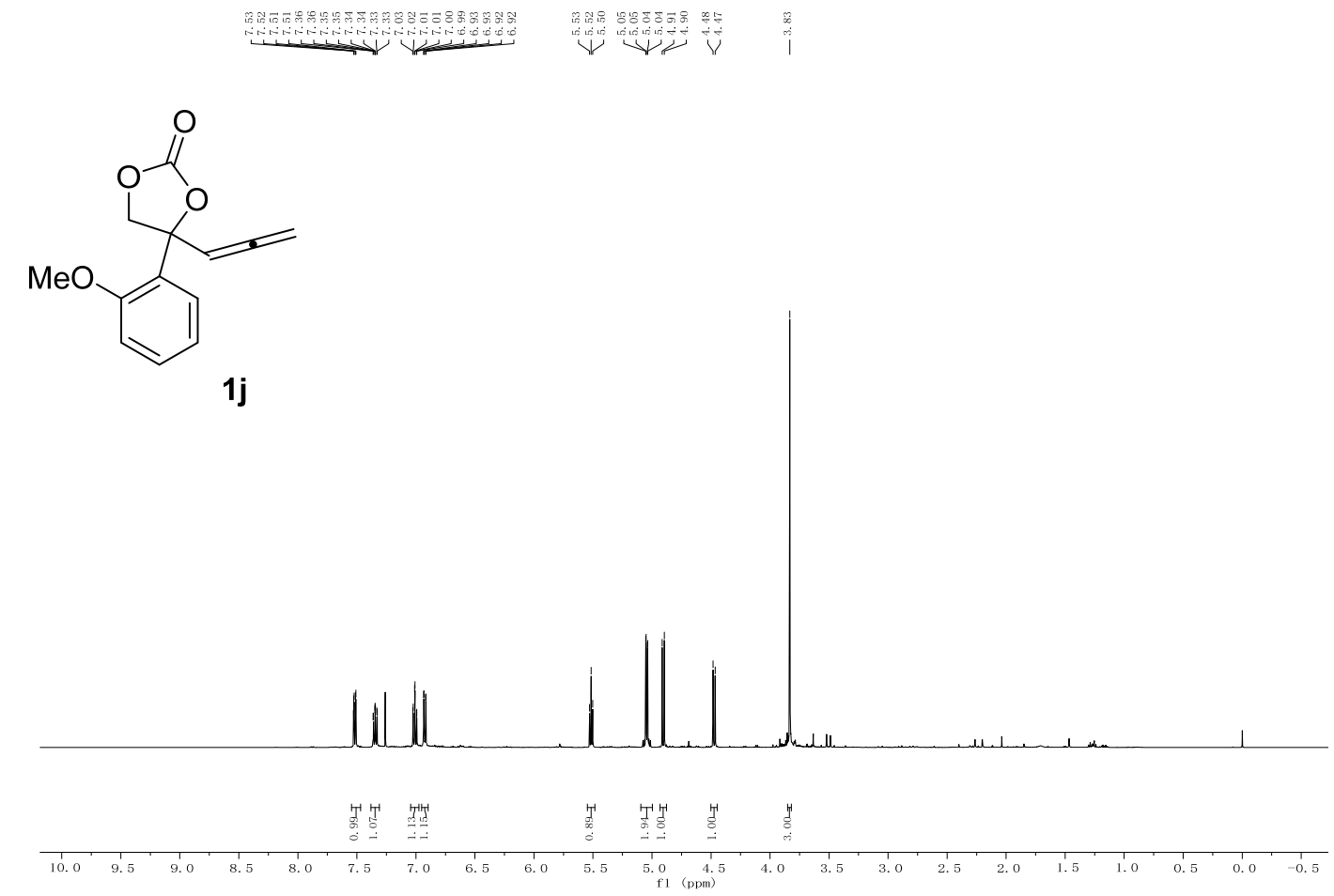

${ }^{13} \mathrm{C}$ NMR spectrum $\left(126 \mathrm{MHz}, \mathrm{CDCl}_{3}\right)$

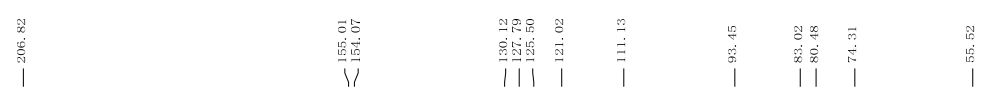
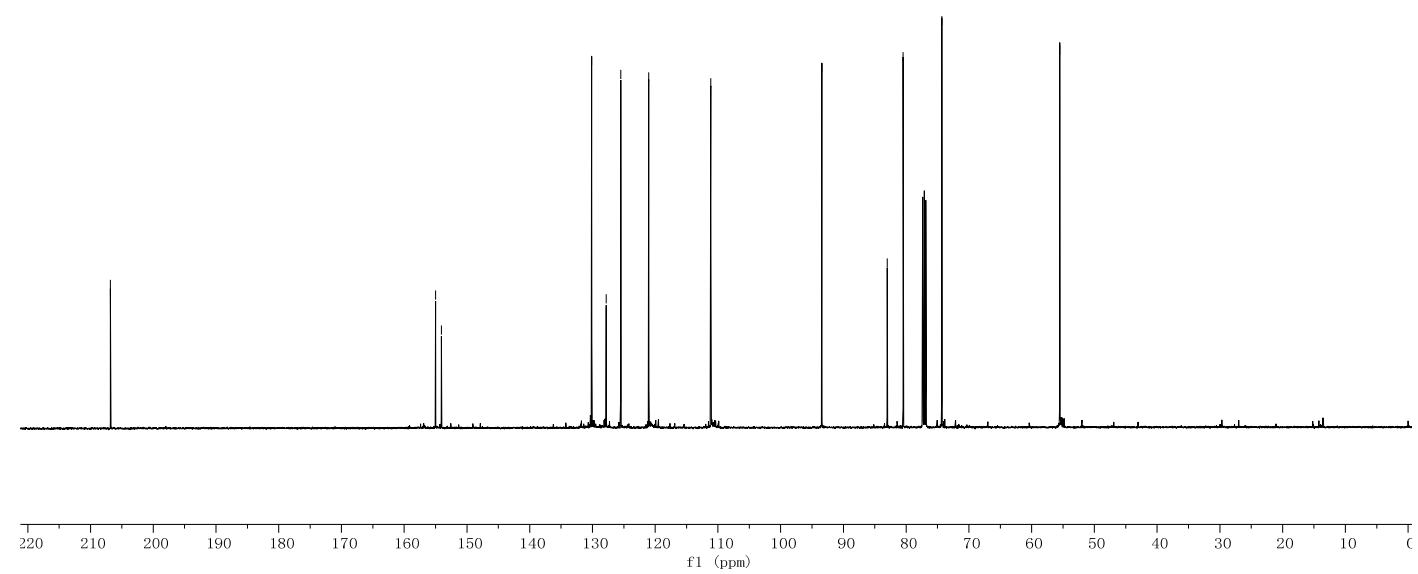
${ }^{1} \mathrm{H}$ NMR spectrum $\left(500 \mathrm{MHz}, \mathrm{CDCl}_{3}\right)$

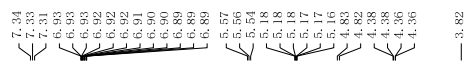<smiles>C=CCC1(c2cccc(OC)c2)COC(=O)O1</smiles>

$1 \mathrm{k}$

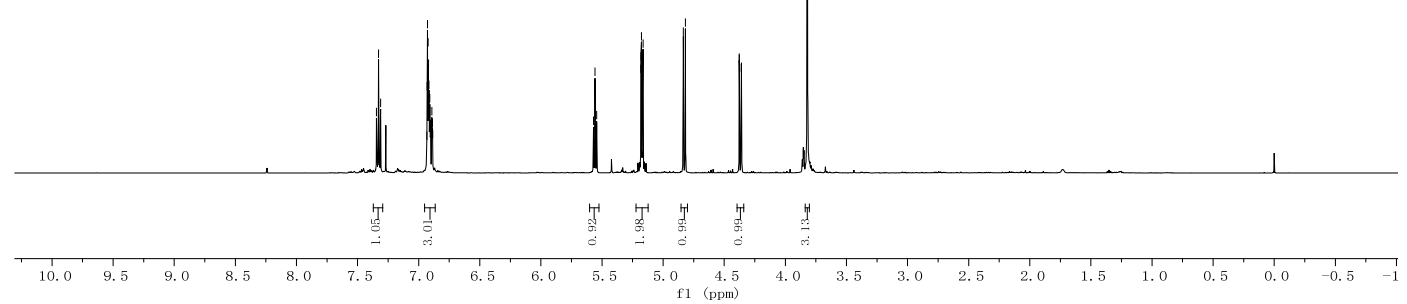

${ }^{13} \mathrm{C}$ NMR spectrum $\left(126 \mathrm{MHz}, \mathrm{CDCl}_{3}\right)$

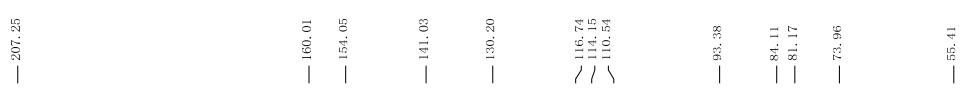
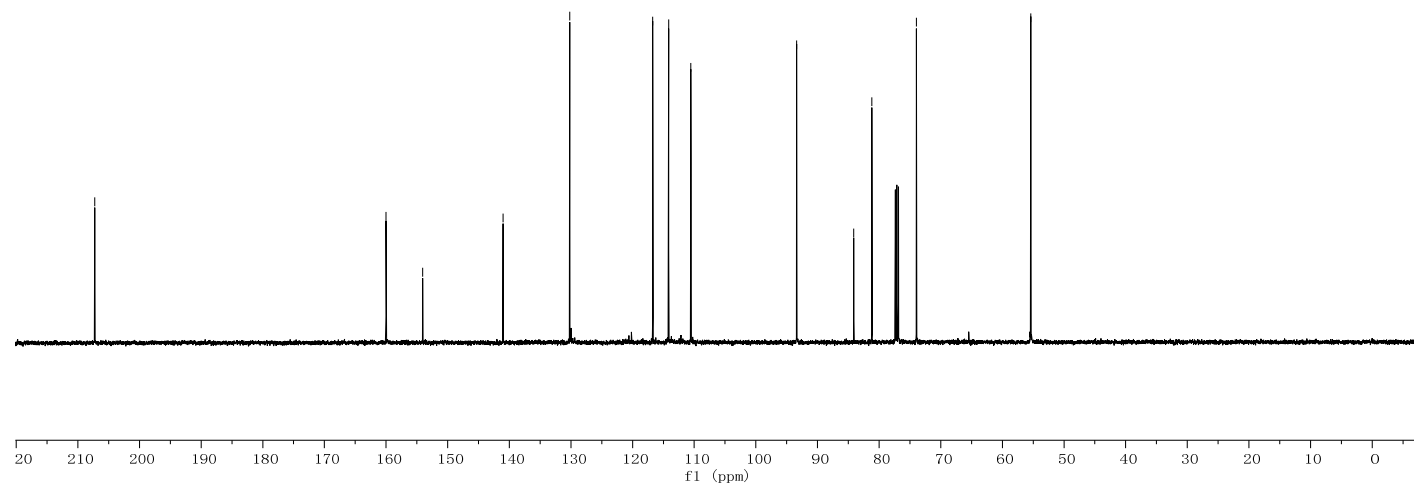
${ }^{1} \mathrm{H}$ NMR spectrum $\left(500 \mathrm{MHz}, \mathrm{CDCl}_{3}\right)$

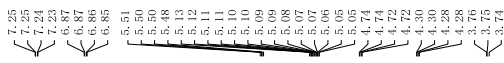

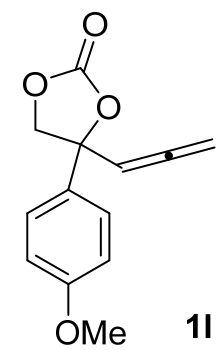

1

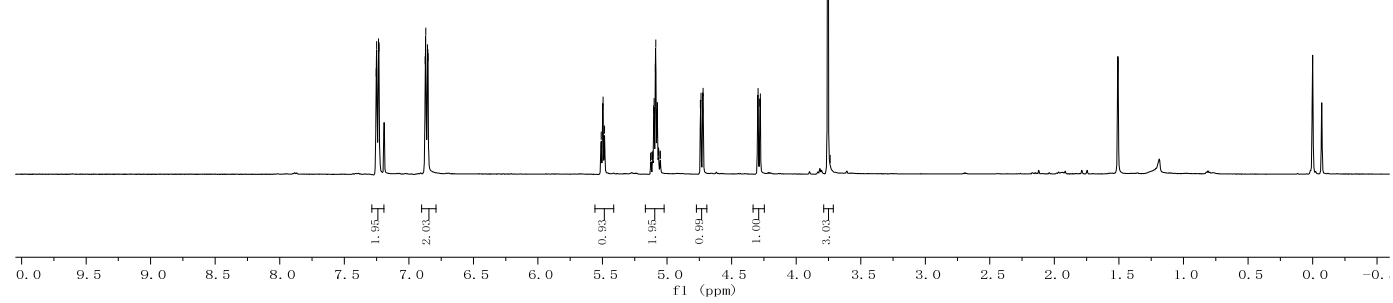

${ }^{13} \mathrm{C}$ NMR spectrum $\left(126 \mathrm{MHz}, \mathrm{CDCl}_{3}\right)$

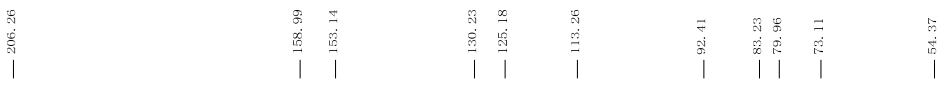

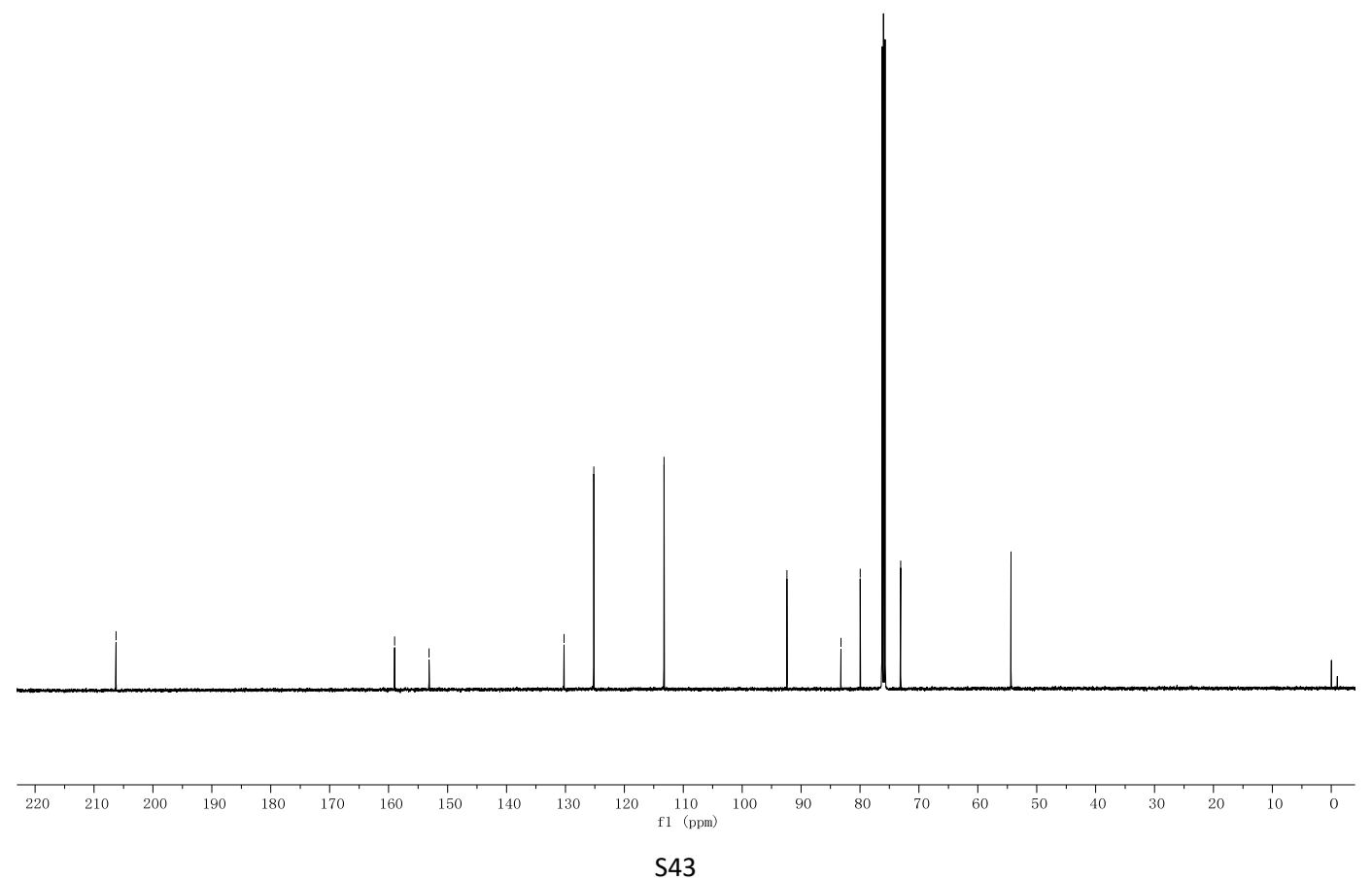


${ }^{1} \mathrm{H}$ NMR spectrum $\left(400 \mathrm{MHz}, \mathrm{CDCl}_{3}\right)$
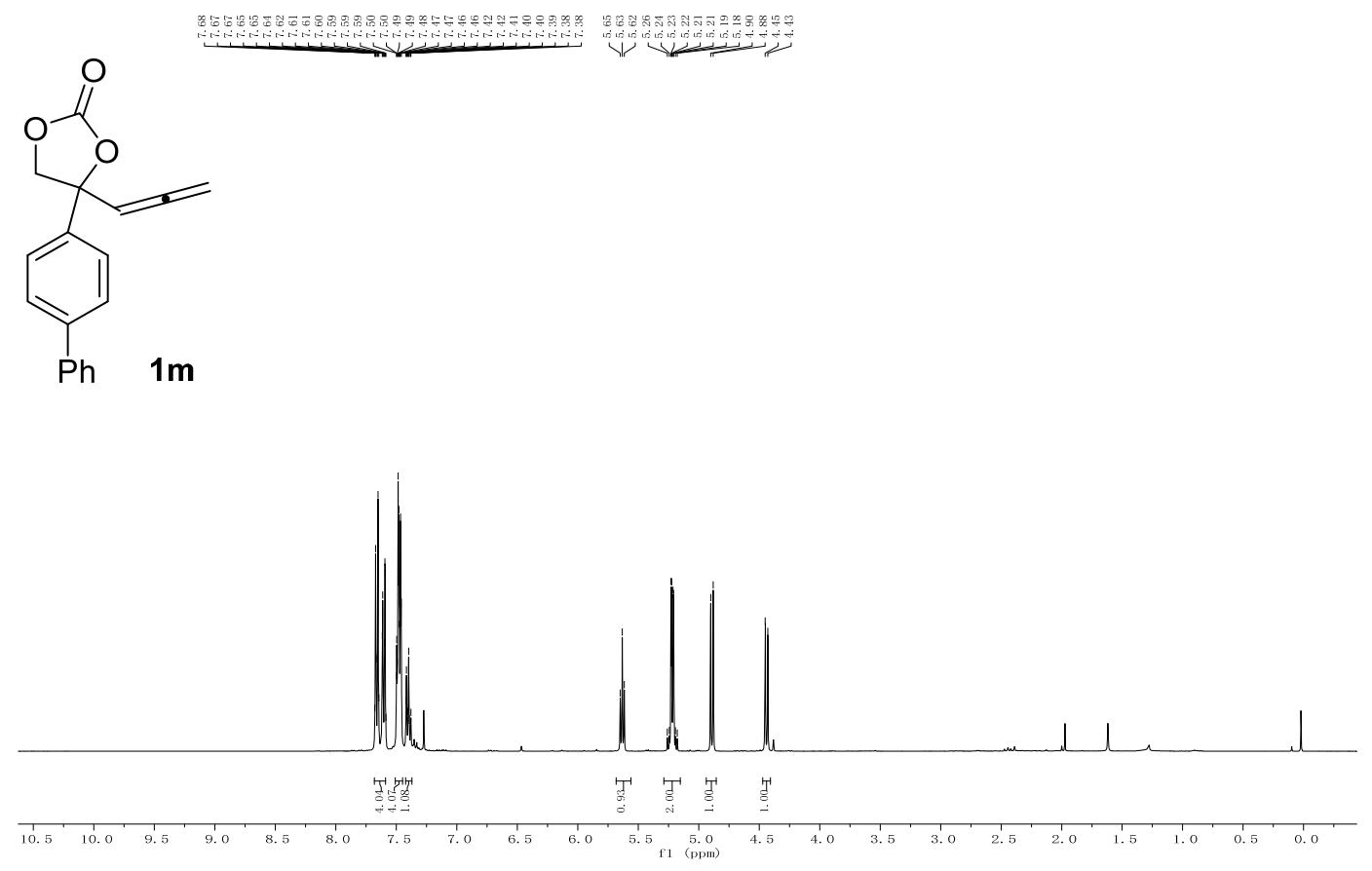

${ }^{13} \mathrm{C}$ NMR spectrum $\left(101 \mathrm{MHz}, \mathrm{CDCl}_{3}\right)$

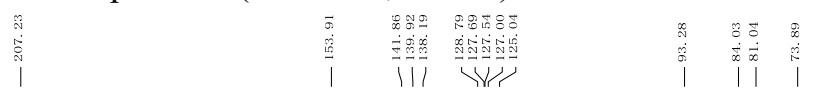
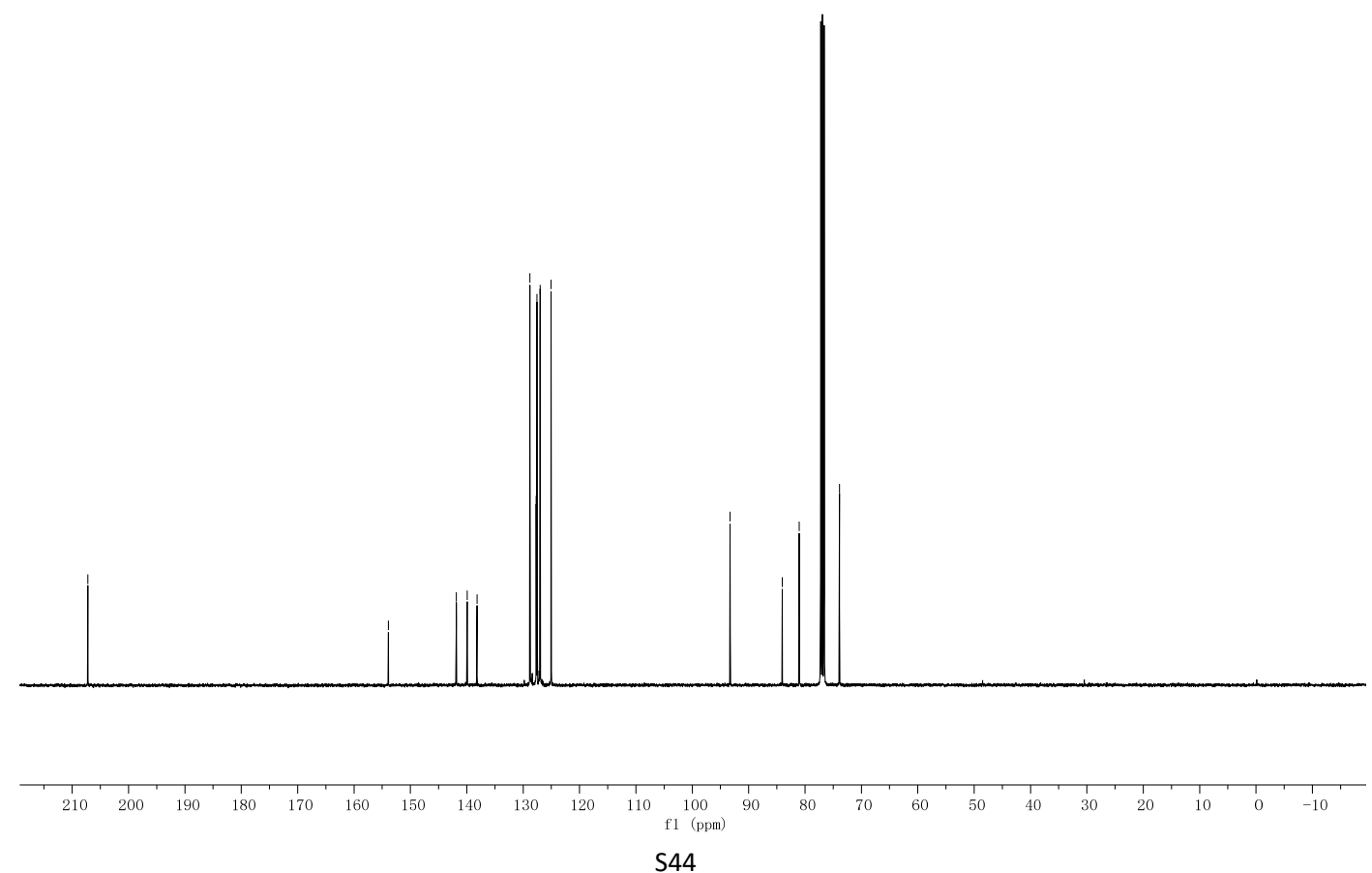
${ }^{1} \mathrm{H}$ NMR spectrum $\left(400 \mathrm{MHz}, \mathrm{CDCl}_{3}\right)$

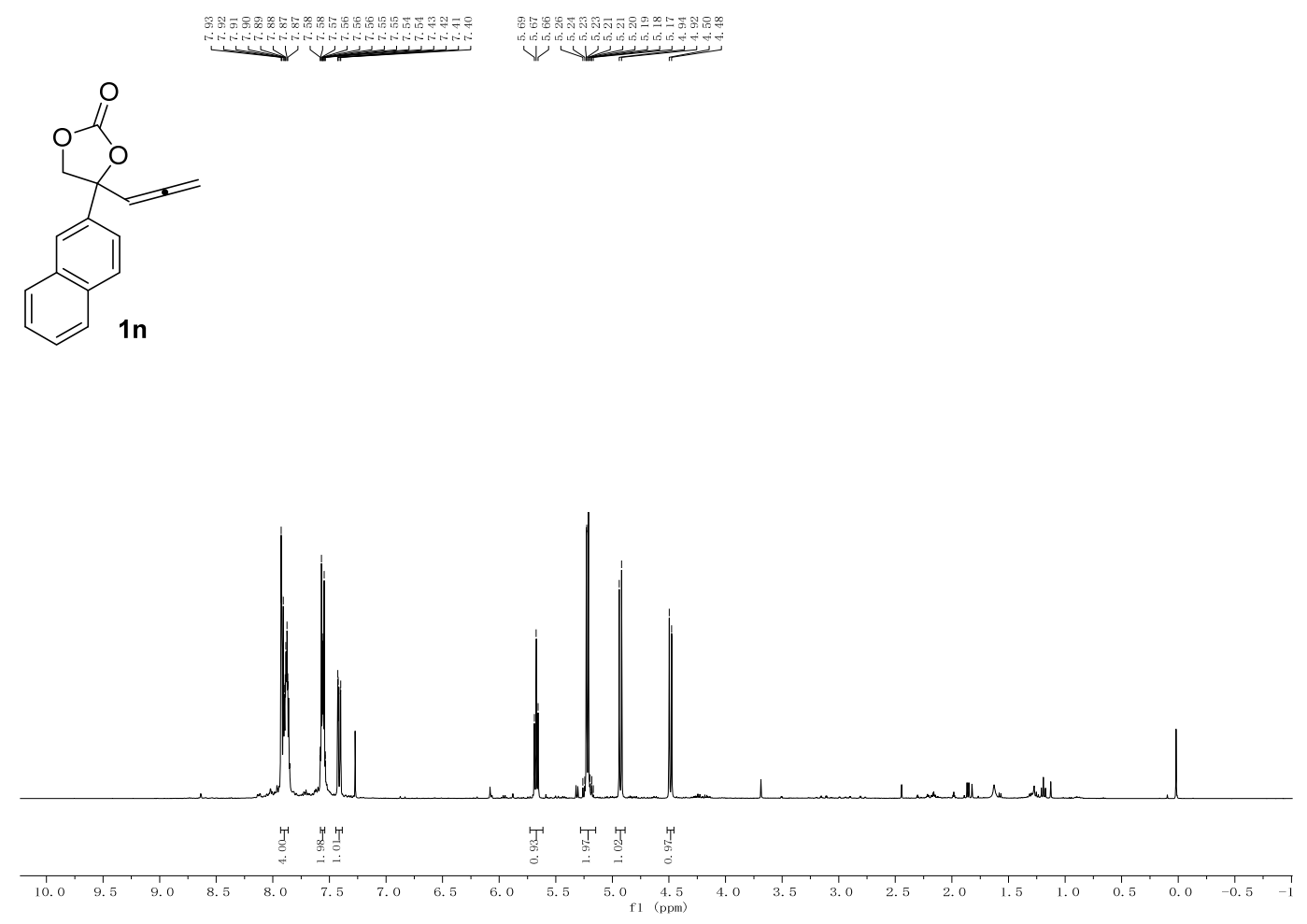

${ }^{13} \mathrm{C}$ NMR spectrum $\left(101 \mathrm{MHz}, \mathrm{CDCl}_{3}\right)$

\begin{tabular}{|c|c|c|}
\hline | & 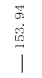 & 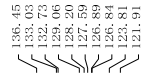 \\
\hline
\end{tabular}
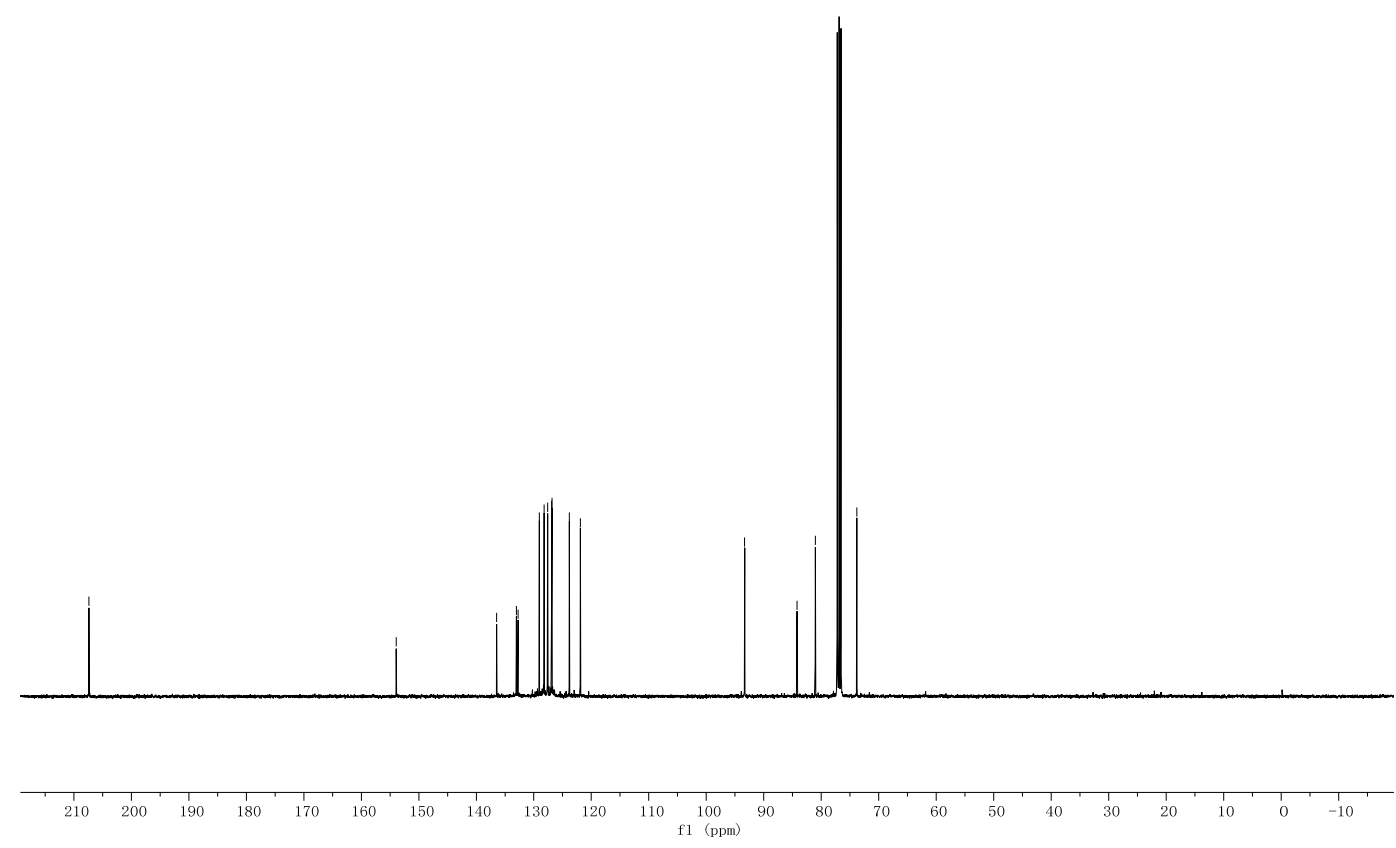
${ }^{1} \mathrm{H}$ NMR spectrum $\left(300 \mathrm{MHz}, \mathrm{CDCl}_{3}\right)$

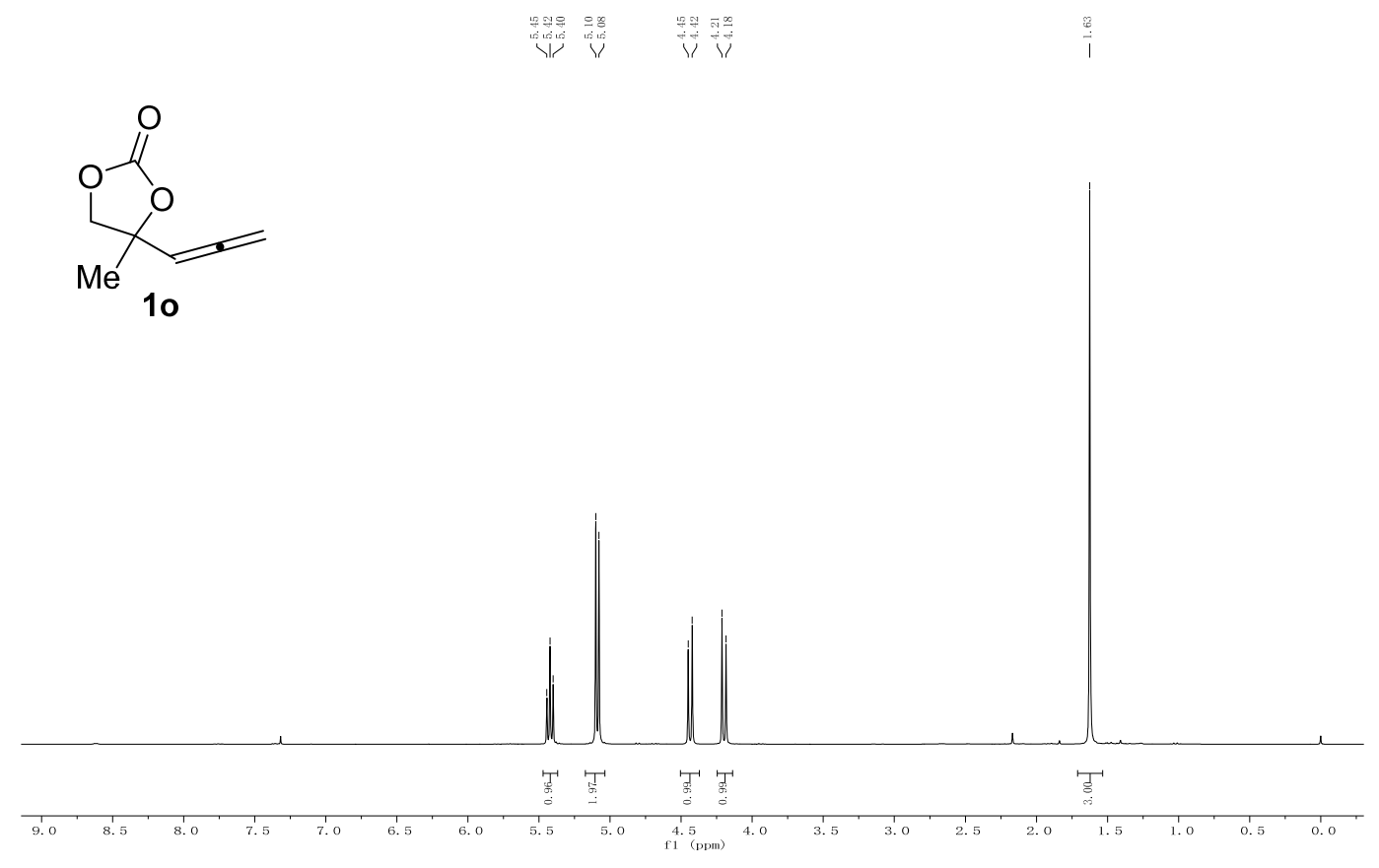

${ }^{13} \mathrm{C}$ NMR spectrum $\left(126 \mathrm{MHz}, \mathrm{CDCl}_{3}\right)$

0
1

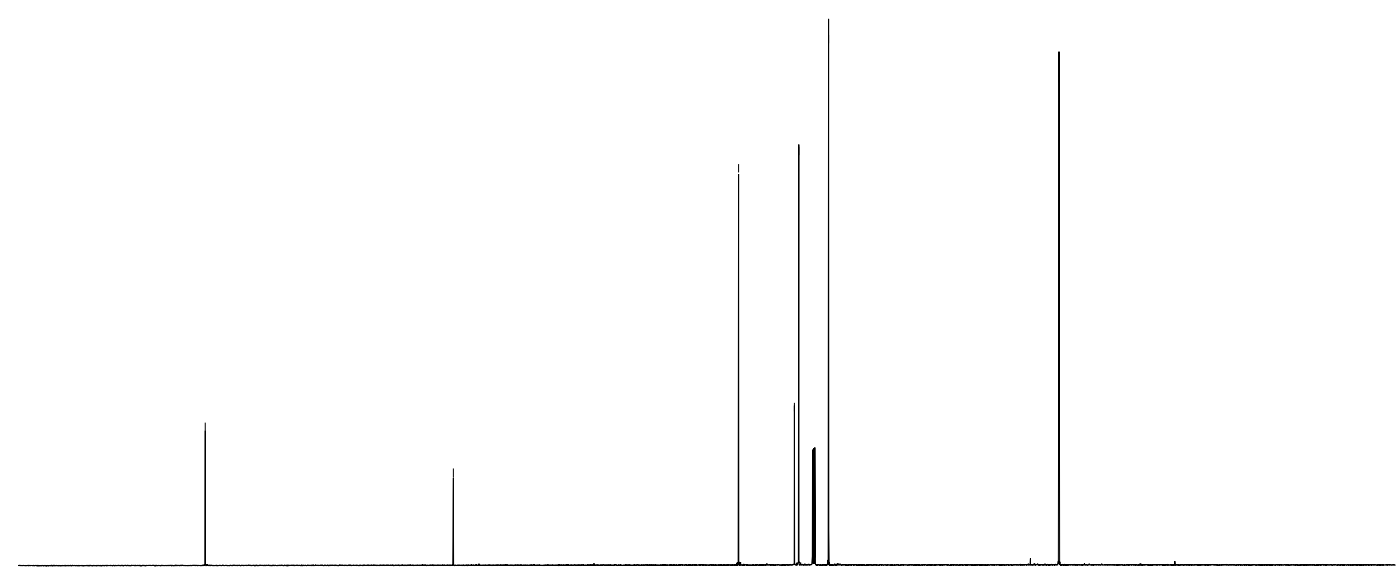

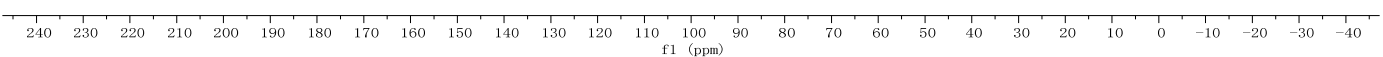


${ }^{1} \mathrm{H}$ NMR spectrum $\left(500 \mathrm{MHz}, \mathrm{CDCl}_{3}\right)$

S5negeseg

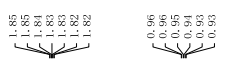
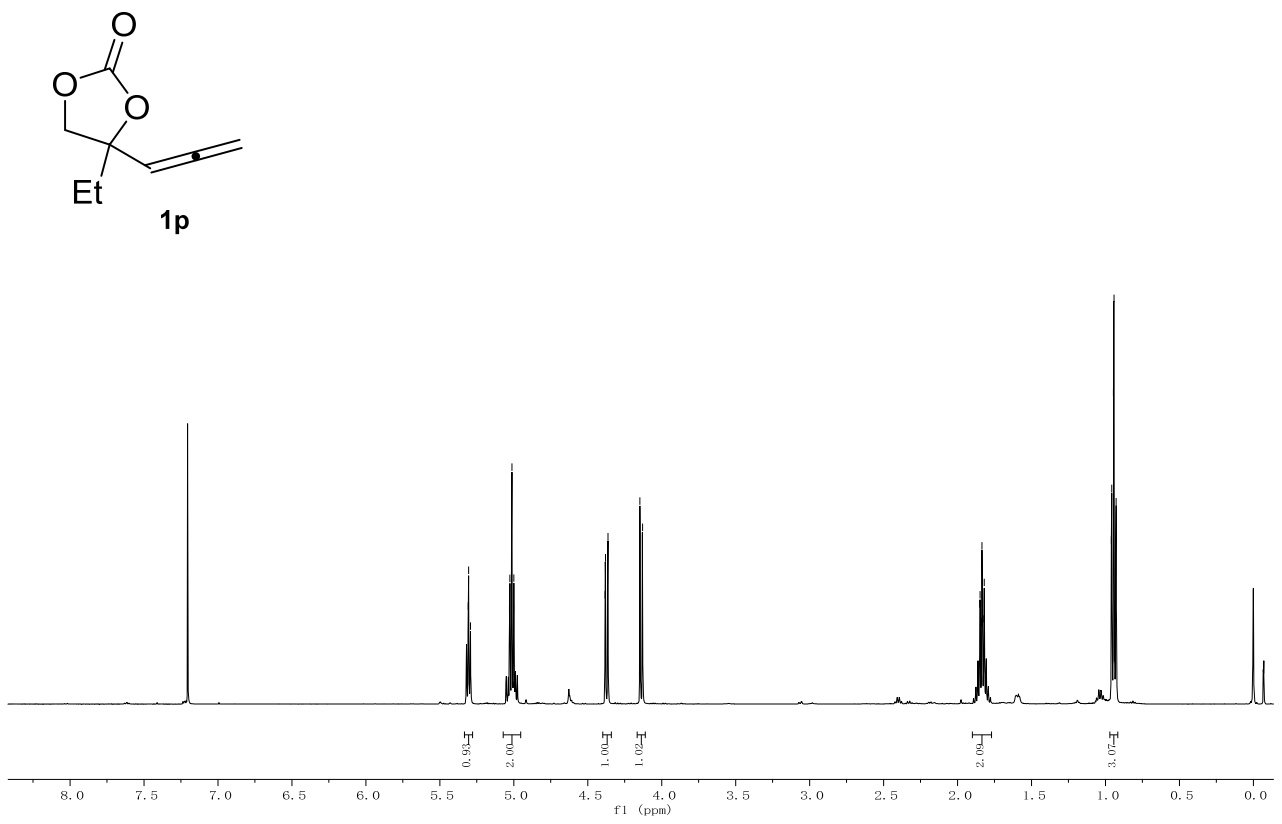

${ }^{13} \mathrm{C}$ NMR spectrum $\left(126 \mathrm{MHz}, \mathrm{CDCl}_{3}\right)$

|

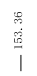

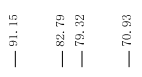
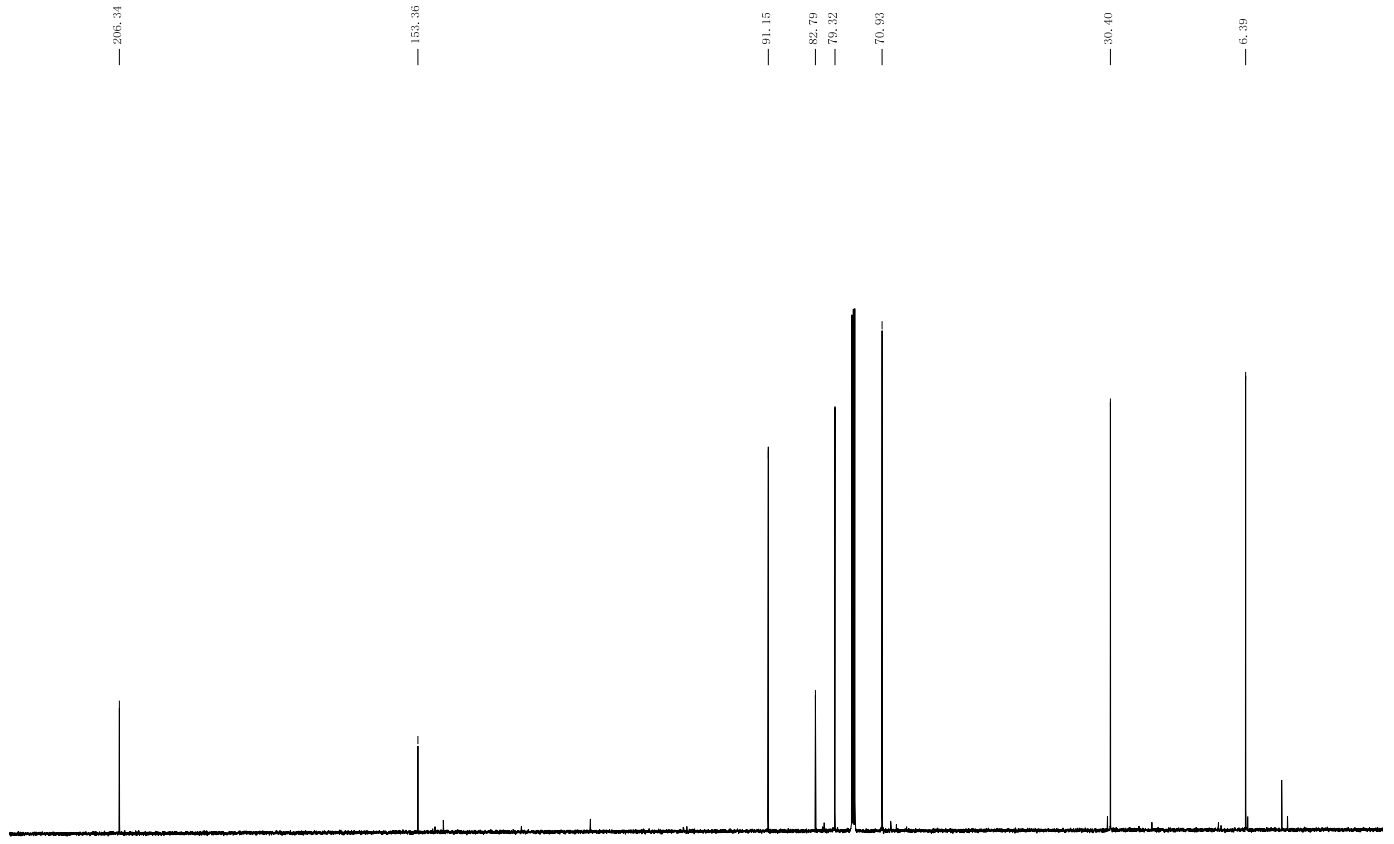

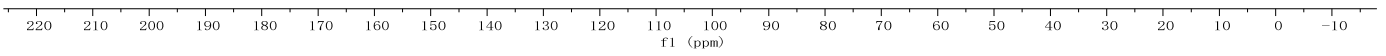

S47 
${ }^{1} \mathrm{H}$ NMR spectrum $\left(500 \mathrm{MHz}, \mathrm{CDCl}_{3}\right)$

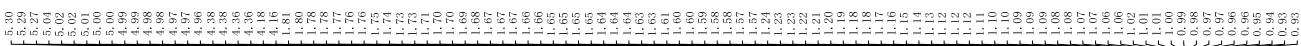

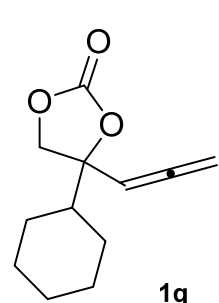

$1 \mathrm{q}$

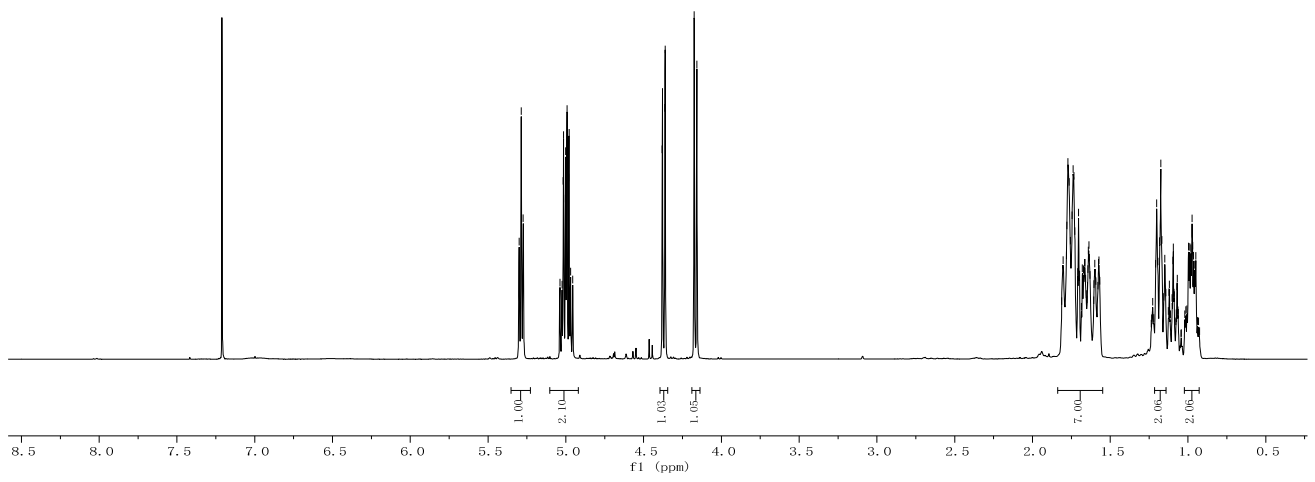

${ }^{13} \mathrm{C} \mathrm{NMR}$ spectrum $\left(126 \mathrm{MHz}, \mathrm{CDCl}_{3}\right)$

1
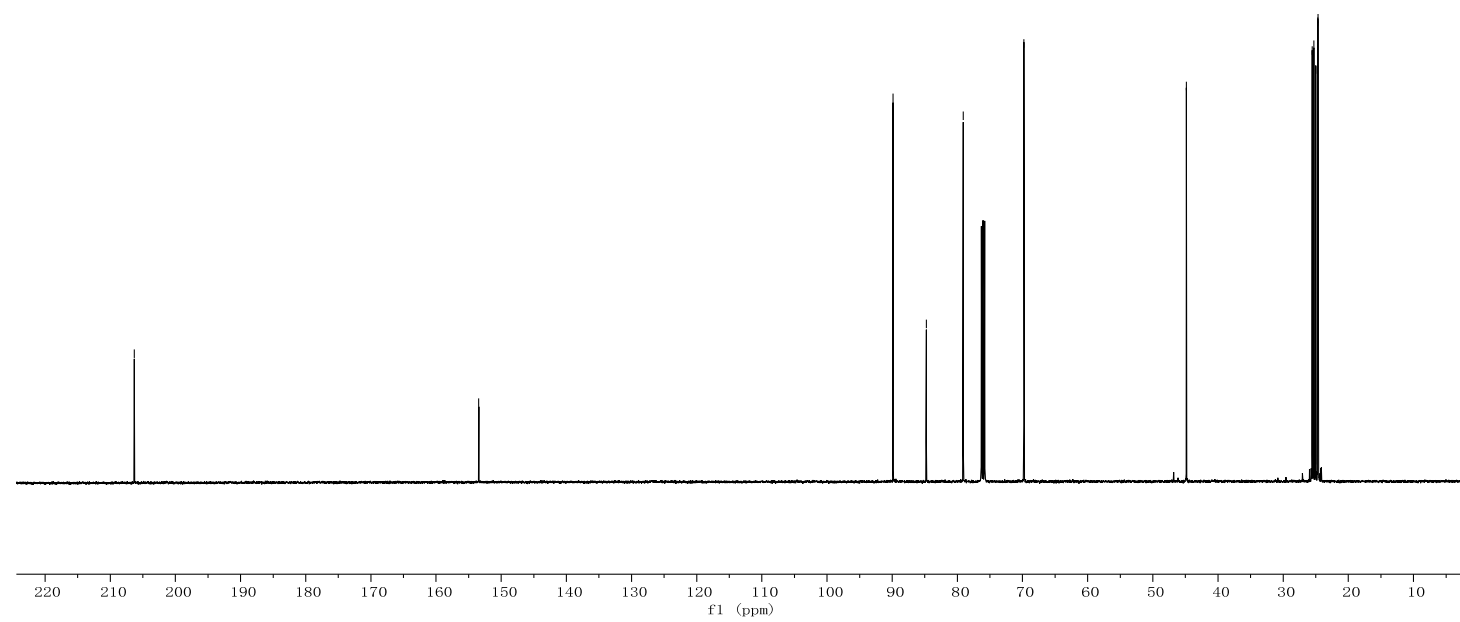
${ }^{1} \mathrm{H}$ NMR spectrum $\left(500 \mathrm{MHz}, \mathrm{CDCl}_{3}\right.$ )

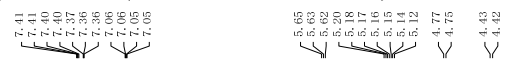<smiles>C=CC1(c2ccsc2)COC(=O)O1</smiles>

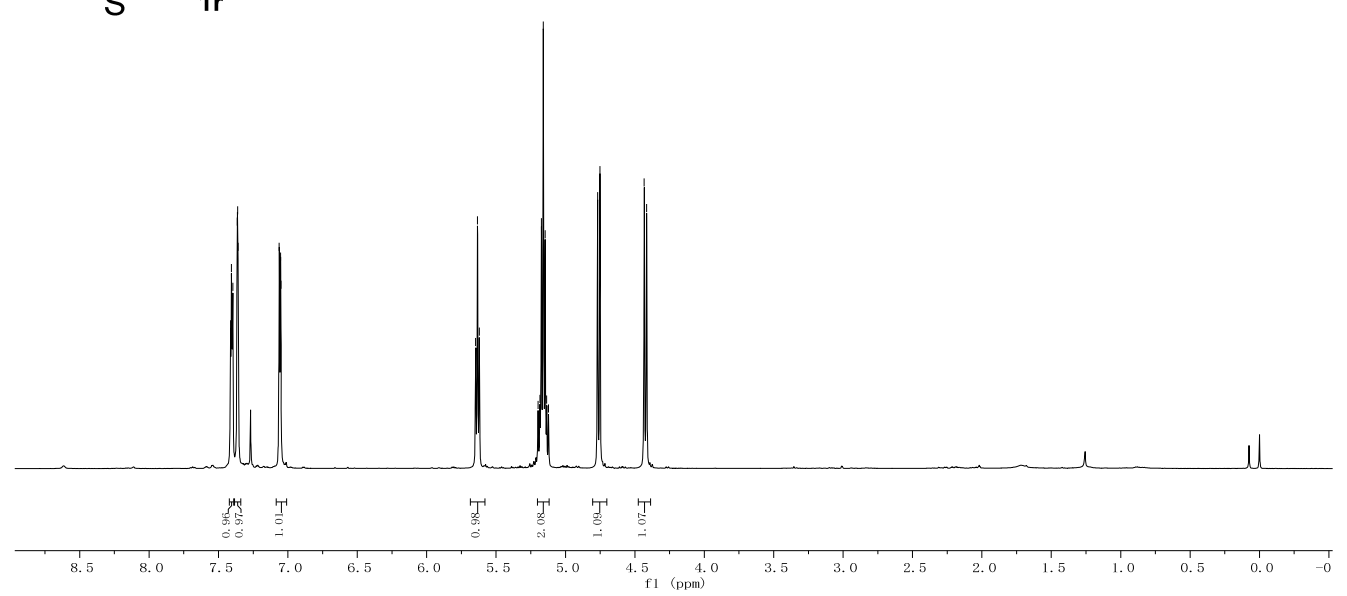

${ }^{13} \mathrm{C}$ NMR spectrum $\left(126 \mathrm{MHz}, \mathrm{CDCl}_{3}\right)$

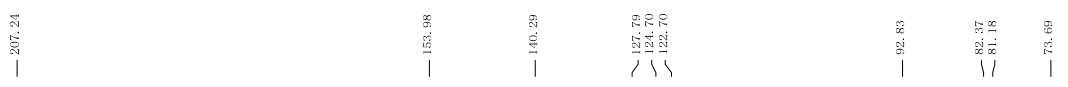

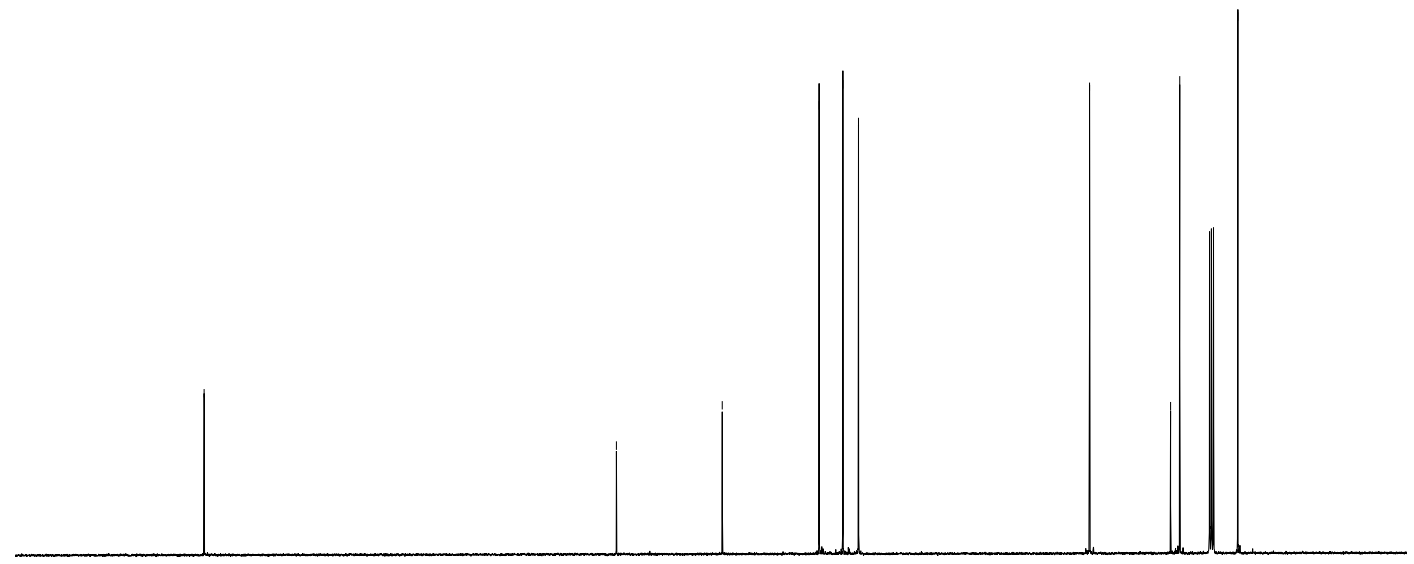

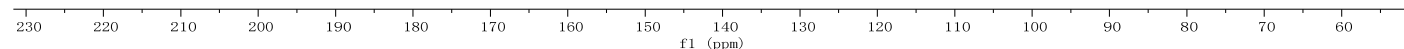
S49 
${ }^{1} \mathrm{H}$ NMR spectrum (400 MHz, $\mathrm{CDCl}_{3}$ )
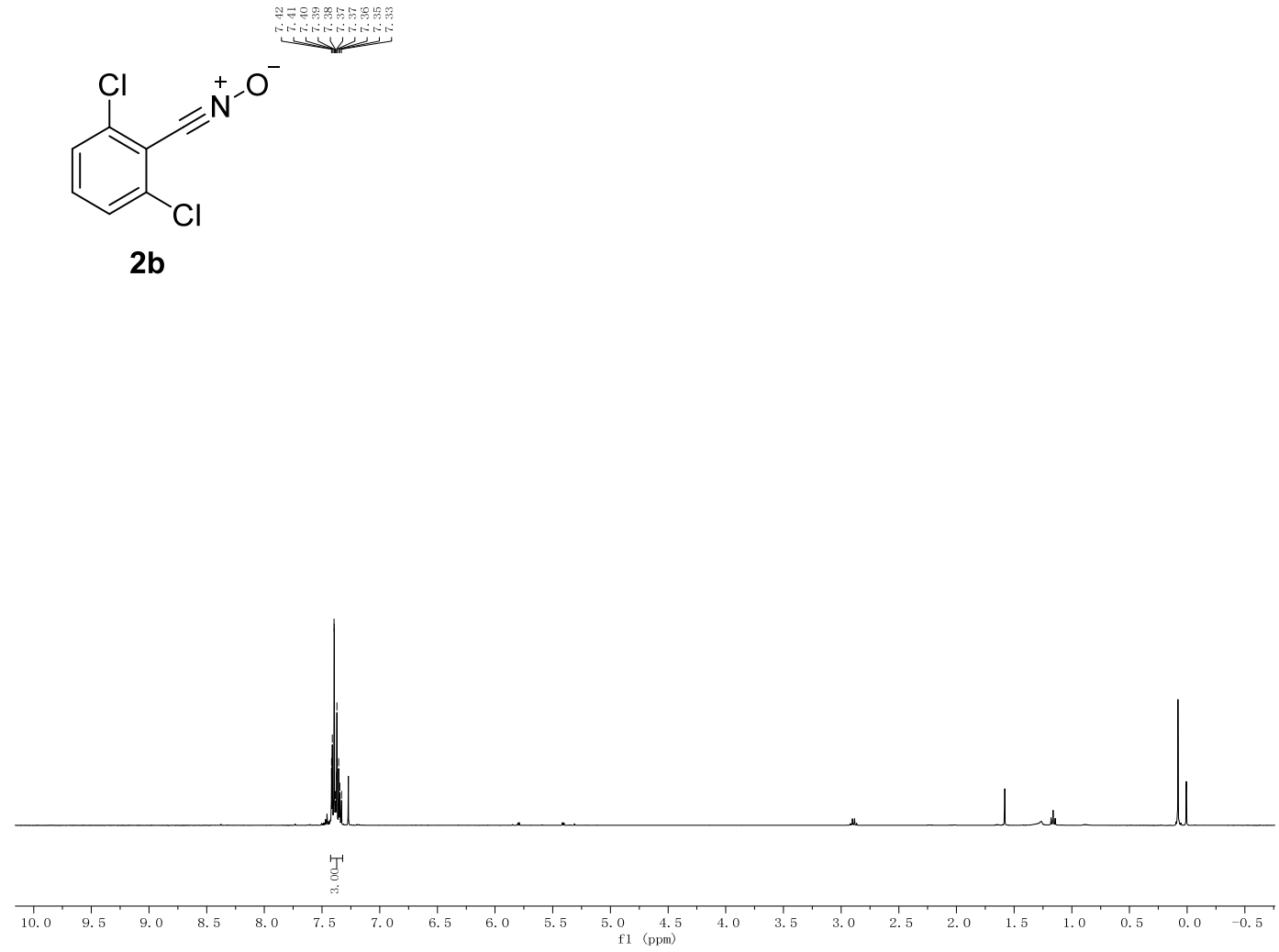

${ }^{13} \mathrm{C}$ NMR spectrum $\left(126 \mathrm{MHz}, \mathrm{CDCl}_{3}\right)$
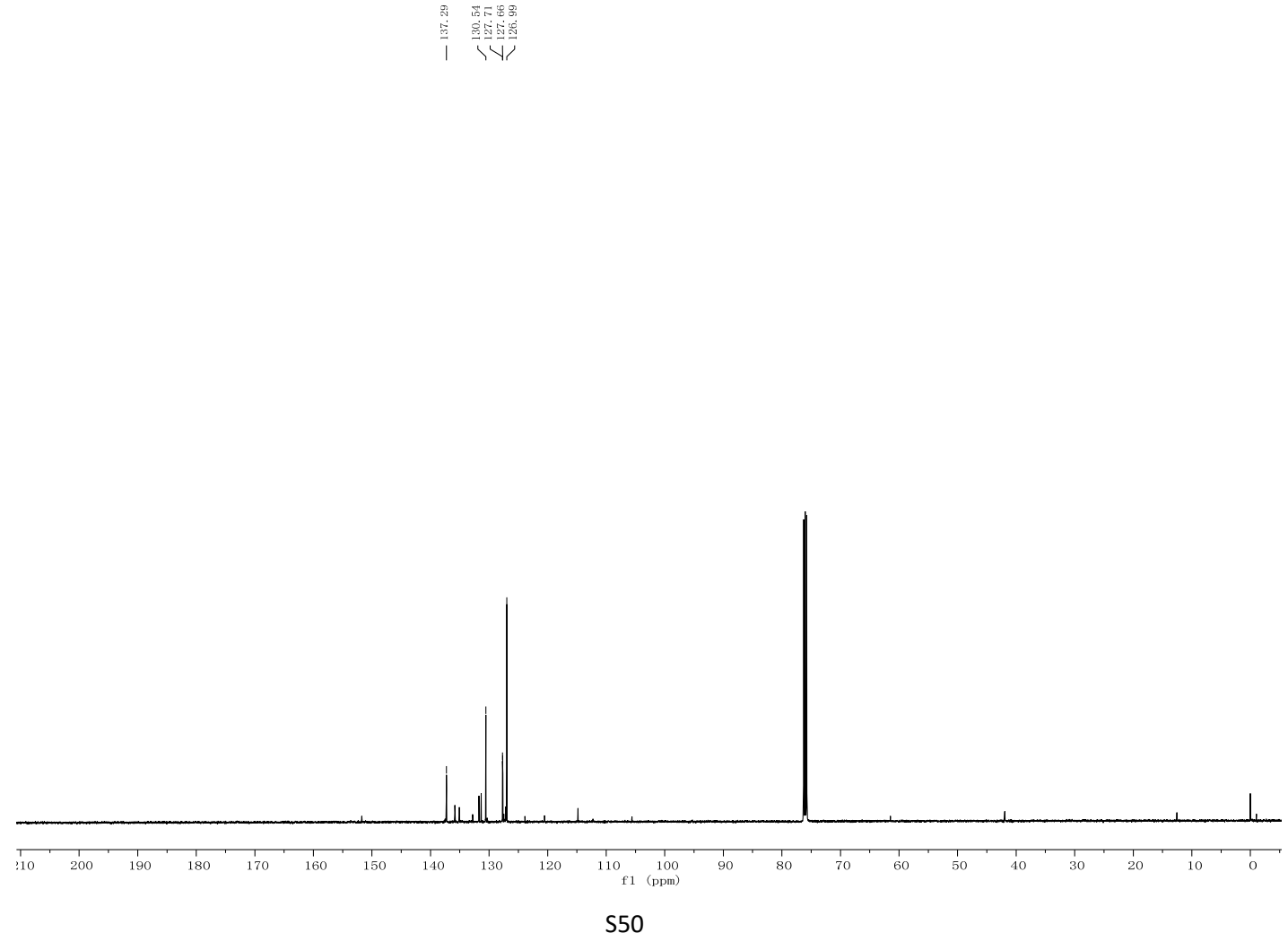
${ }^{1} \mathrm{H}$ NMR spectrum (500 MHz, $\mathrm{CDCl}_{3}$ )

踡
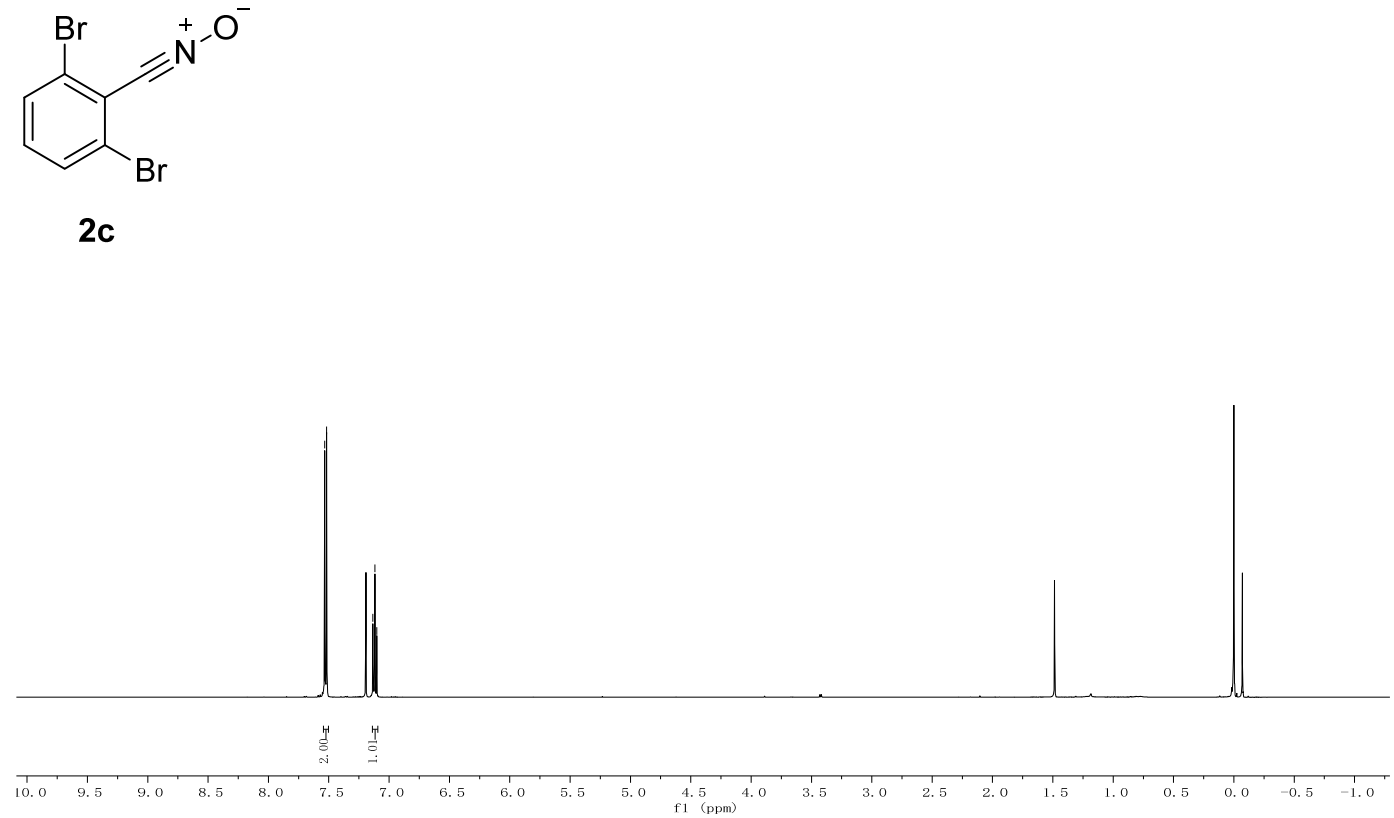

${ }^{13} \mathrm{C}$ NMR spectrum $\left(126 \mathrm{MHz}, \mathrm{CDCl}_{3}\right)$

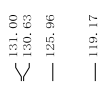

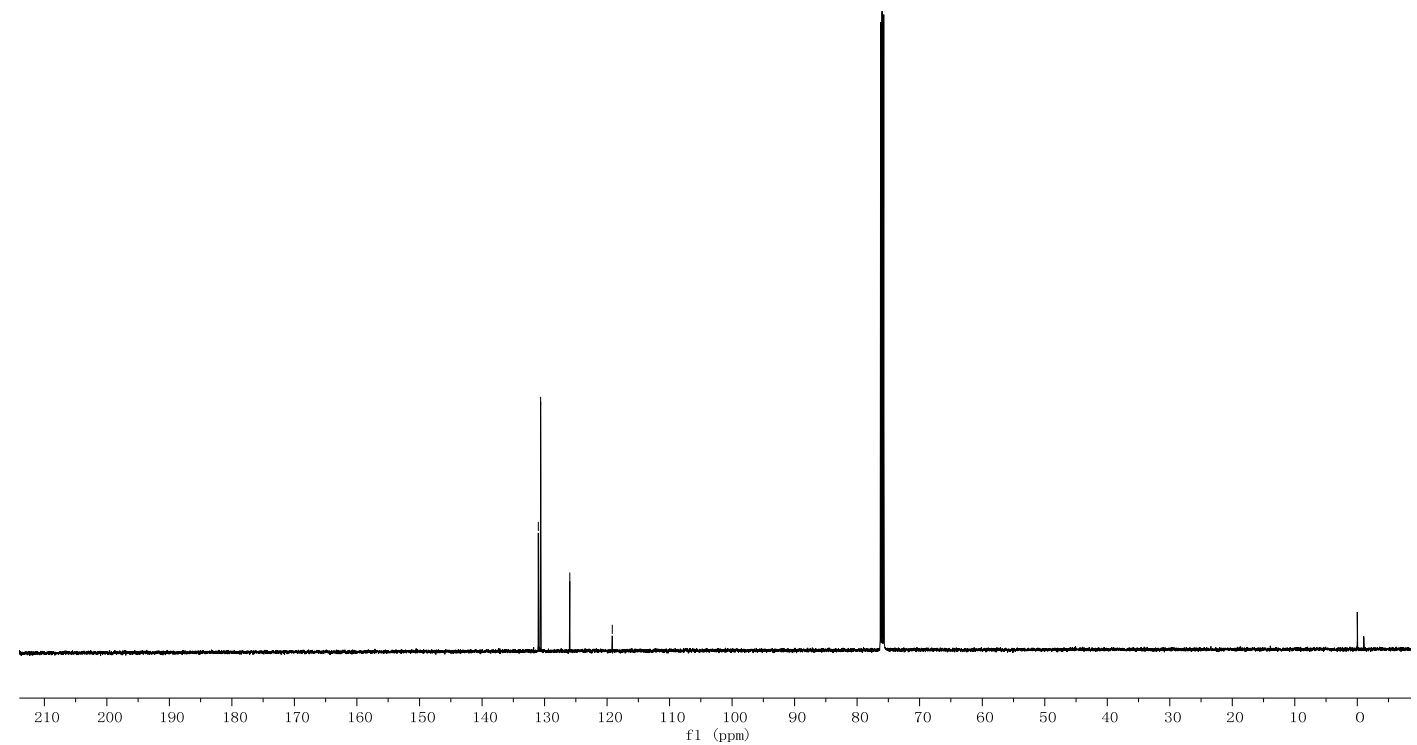

S51 
${ }^{1} \mathrm{H}$ NMR spectrum $\left(500 \mathrm{MHz}, \mathrm{CDCl}_{3}\right)$<smiles>COc1cccc(OC)c1C#[N+][O-]</smiles>

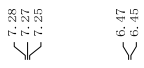

2d

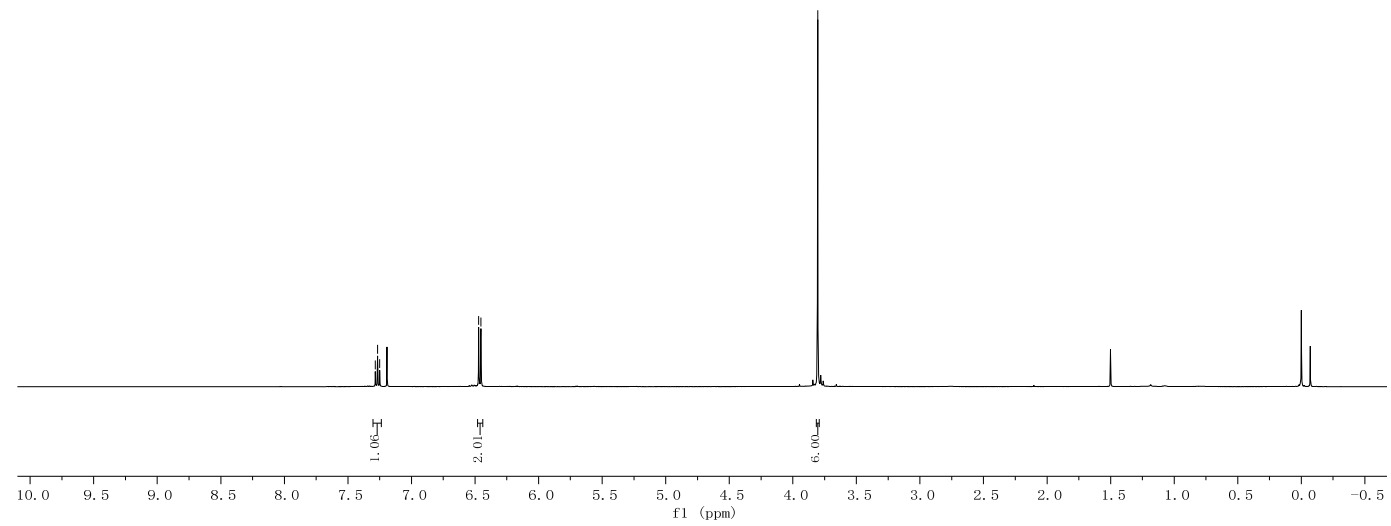

${ }^{13} \mathrm{C}$ NMR spectrum $\left(126 \mathrm{MHz}, \mathrm{CDCl}_{3}\right)$

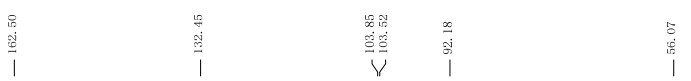

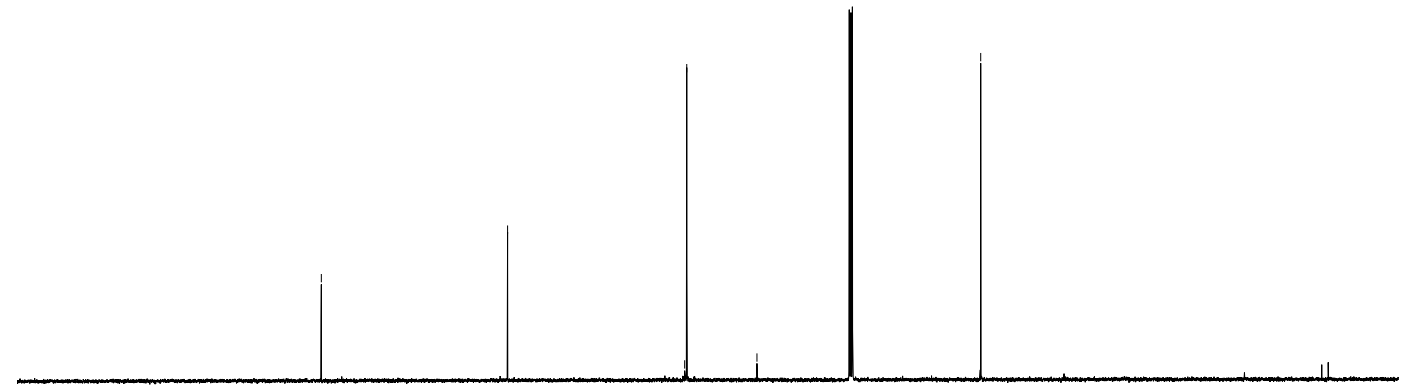

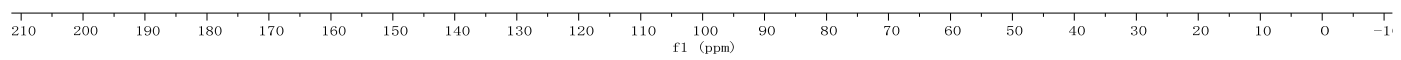


${ }^{1} \mathrm{H}$ NMR spectrum (400 MHz, $\mathrm{CDCl}_{3}$ )
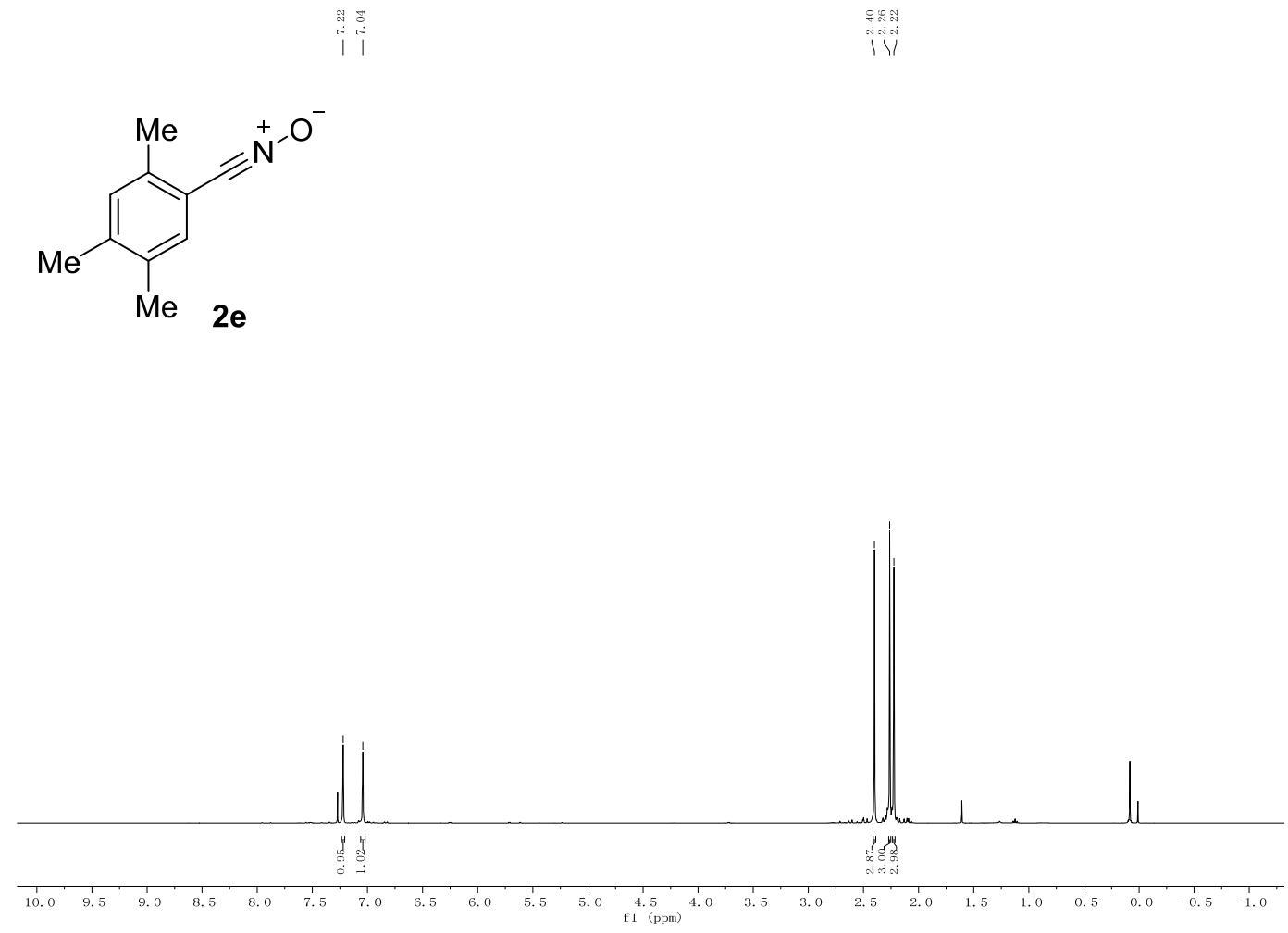

${ }^{13} \mathrm{C}$ NMR spectrum (126 MHz, $\left.\mathrm{CDCl}_{3}\right)$

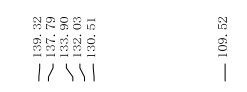

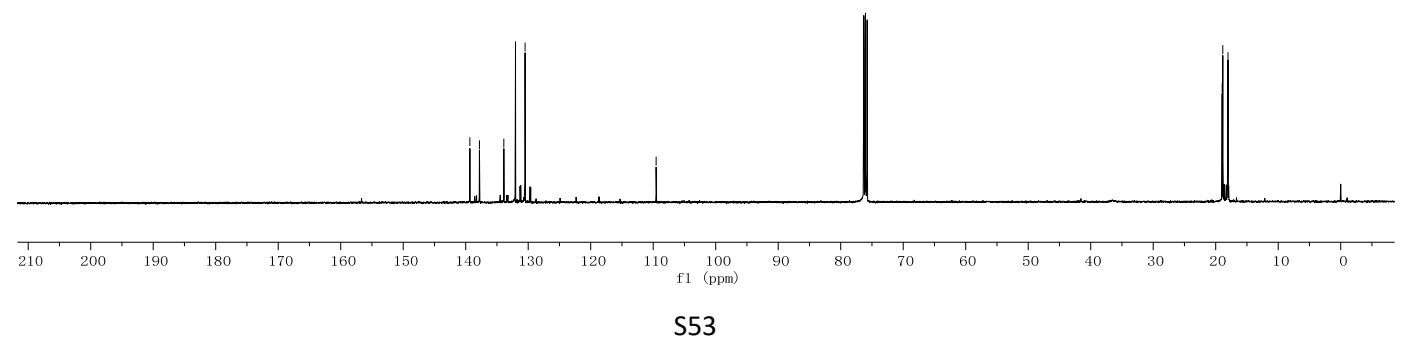


${ }^{1} \mathrm{H}$ NMR spectrum (500 MHz, $\mathrm{CDCl}_{3}$ )
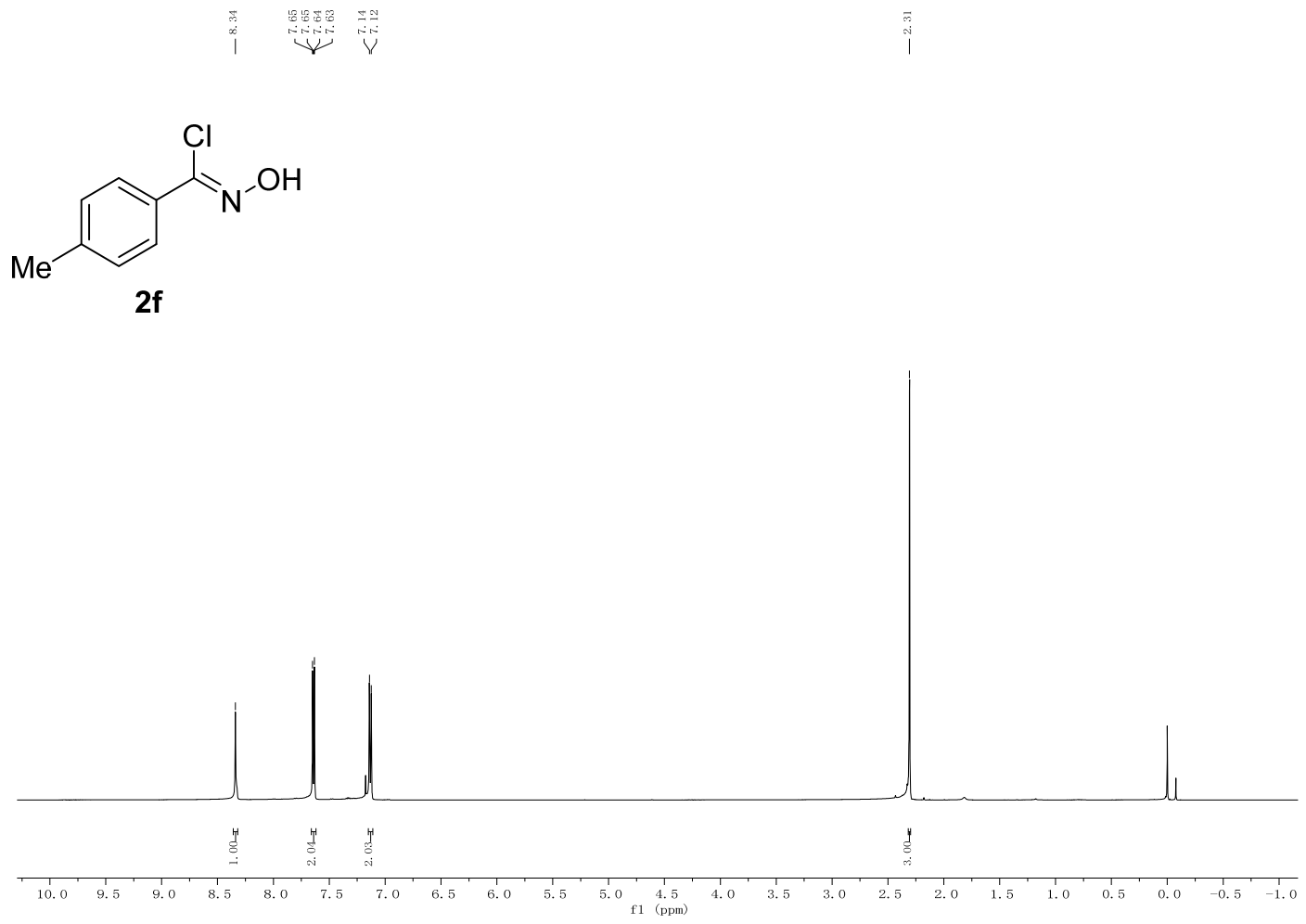

${ }^{13} \mathrm{C}$ NMR spectrum $\left(126 \mathrm{MHz}, \mathrm{CDCl}_{3}\right)$
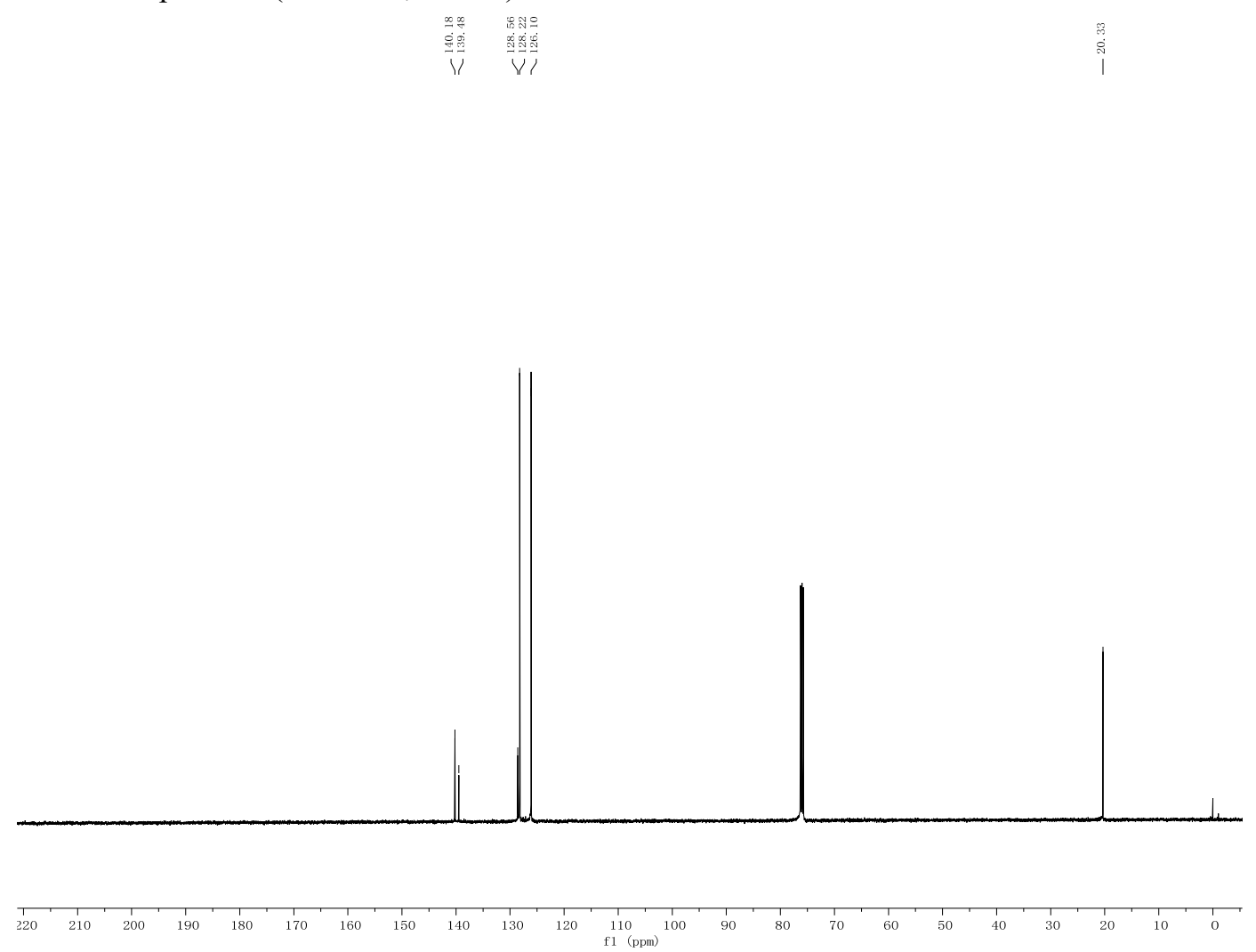
${ }^{1} \mathrm{H}$ NMR spectrum $\left(500 \mathrm{MHz}, \mathrm{CDCl}_{3}\right.$ )

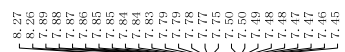
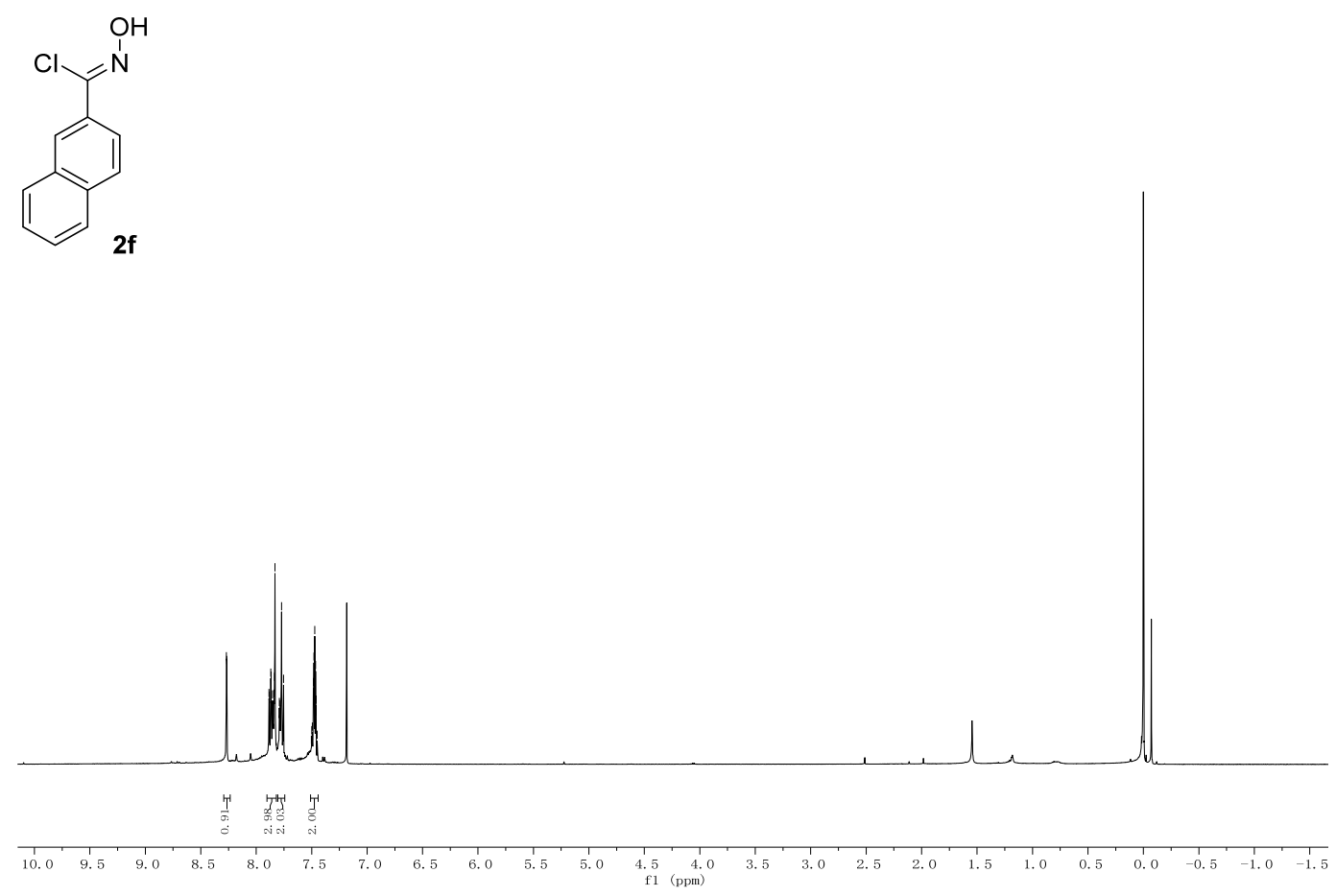

${ }^{13} \mathrm{C}$ NMR spectrum $\left(126 \mathrm{MHz}, \mathrm{CDCl}_{3}\right)$

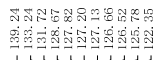

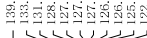

14
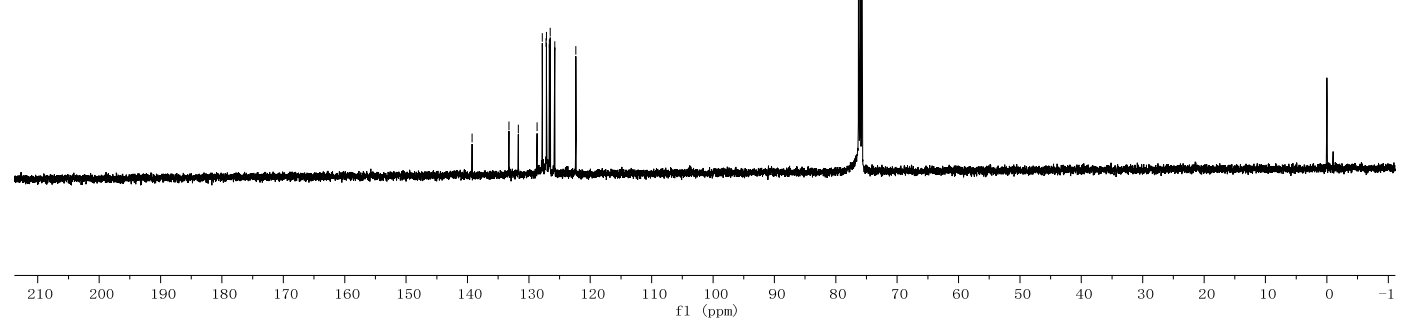
${ }^{1} \mathrm{H}$ and ${ }^{13} \mathrm{C}$ NMR Spectra of All Products

${ }^{1} \mathrm{H}$ NMR spectrum $\left(500 \mathrm{MHz}, \mathrm{CDCl}_{3}\right)$
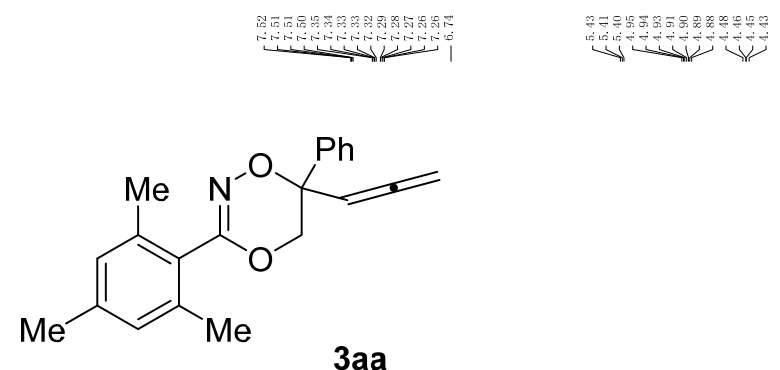

3aa
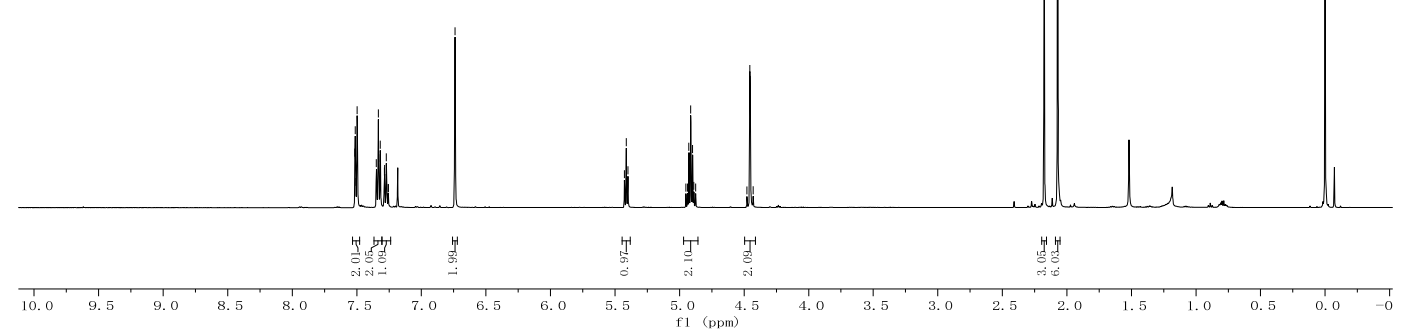

${ }^{13} \mathrm{C}$ NMR spectrum $\left(126 \mathrm{MHz}, \mathrm{CDCl}_{3}\right)$

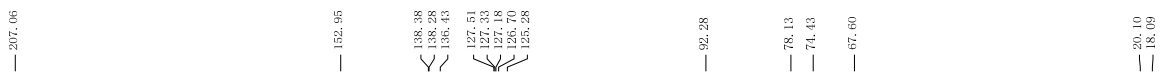

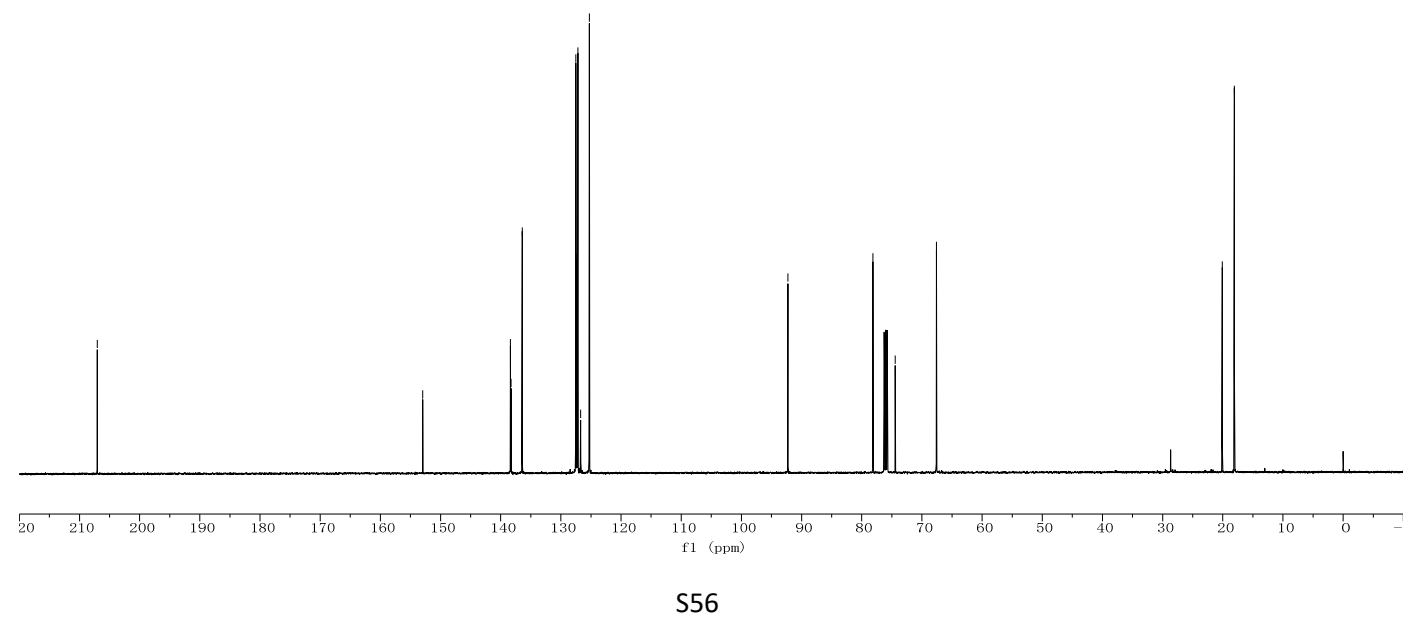


${ }^{1} \mathrm{H}$ NMR spectrum $\left(400 \mathrm{MHz}, \mathrm{CDCl}_{3}\right)$
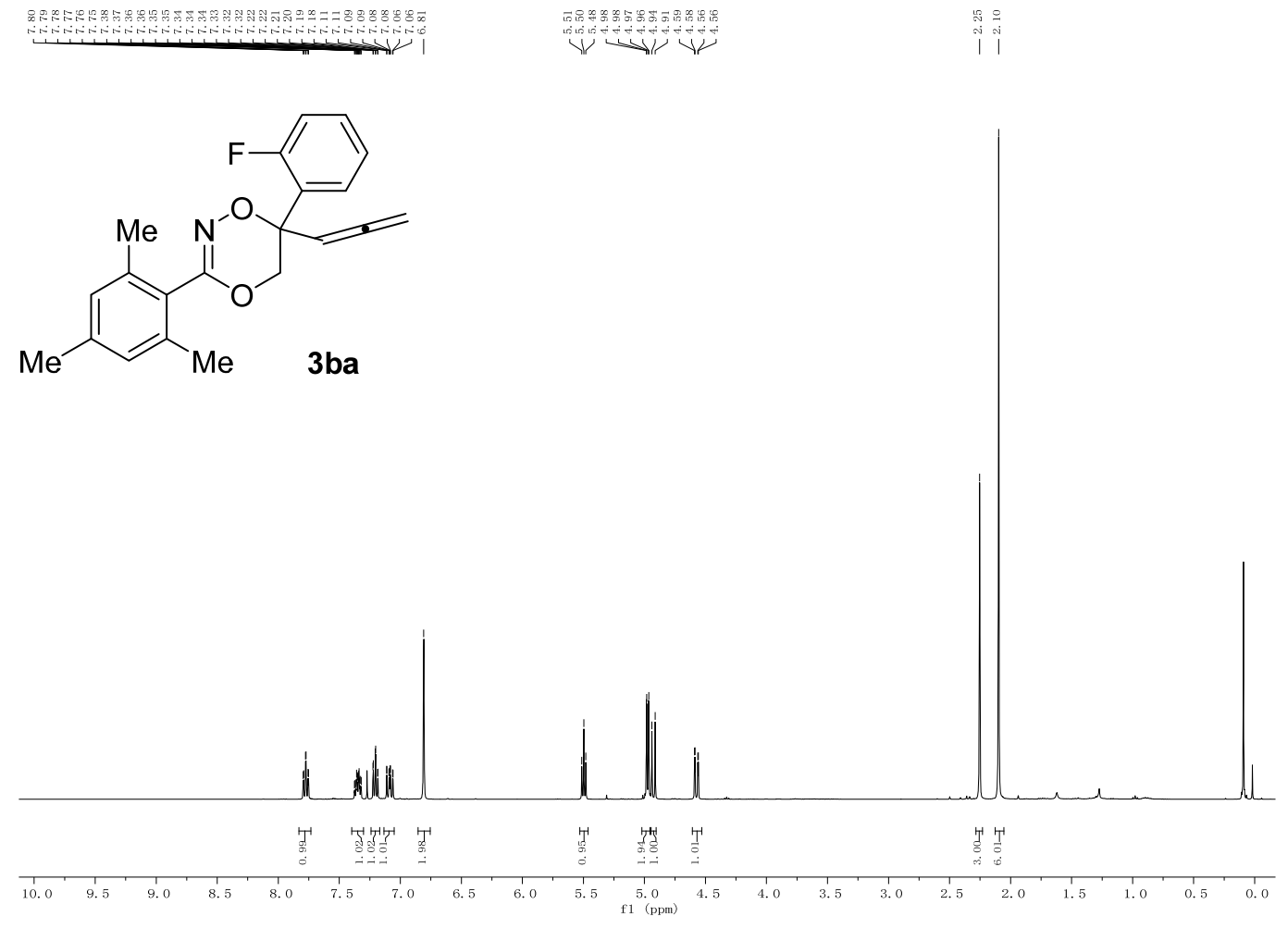

${ }^{13} \mathrm{C}$ NMR spectrum (101 MHz, $\mathrm{CDCl}_{3}$ )

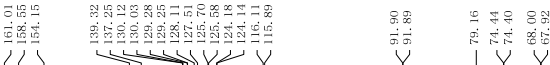

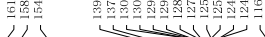

४

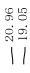

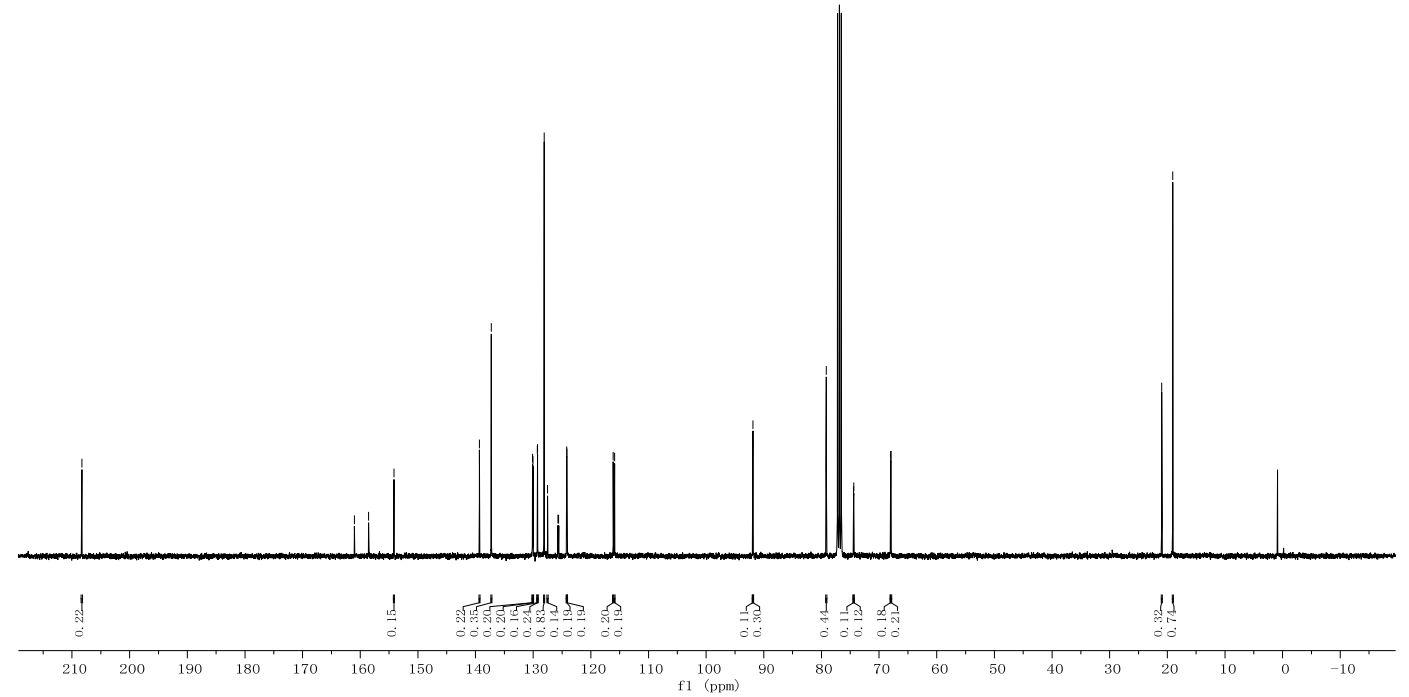


${ }^{1} \mathrm{H}$ NMR spectrum (400 MHz, $\mathrm{CDCl}_{3}$ )

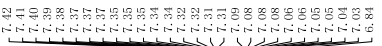
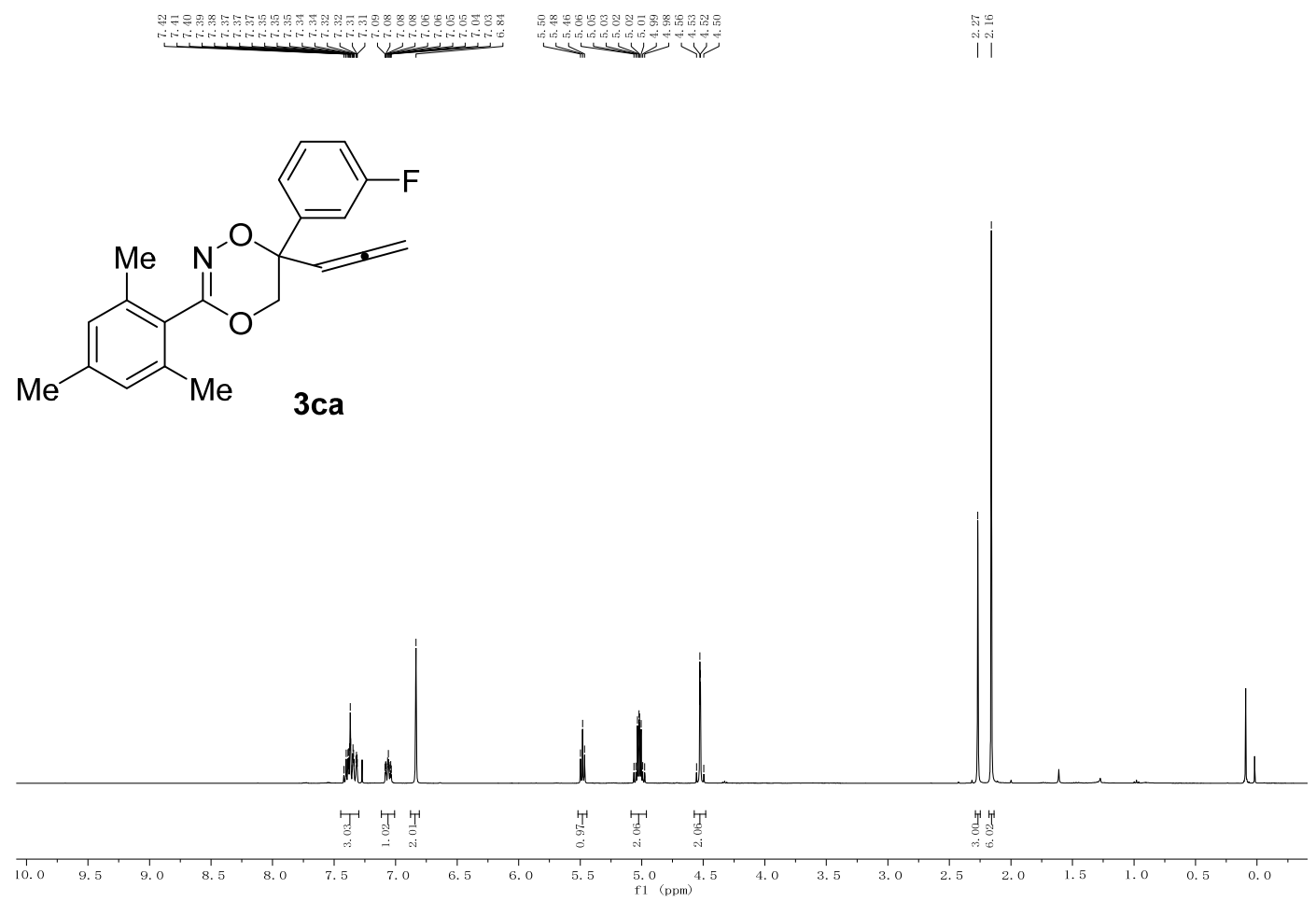

${ }^{13} \mathrm{C}$ NMR spectrum (101 MHz, $\mathrm{CDCl}_{3}$ )
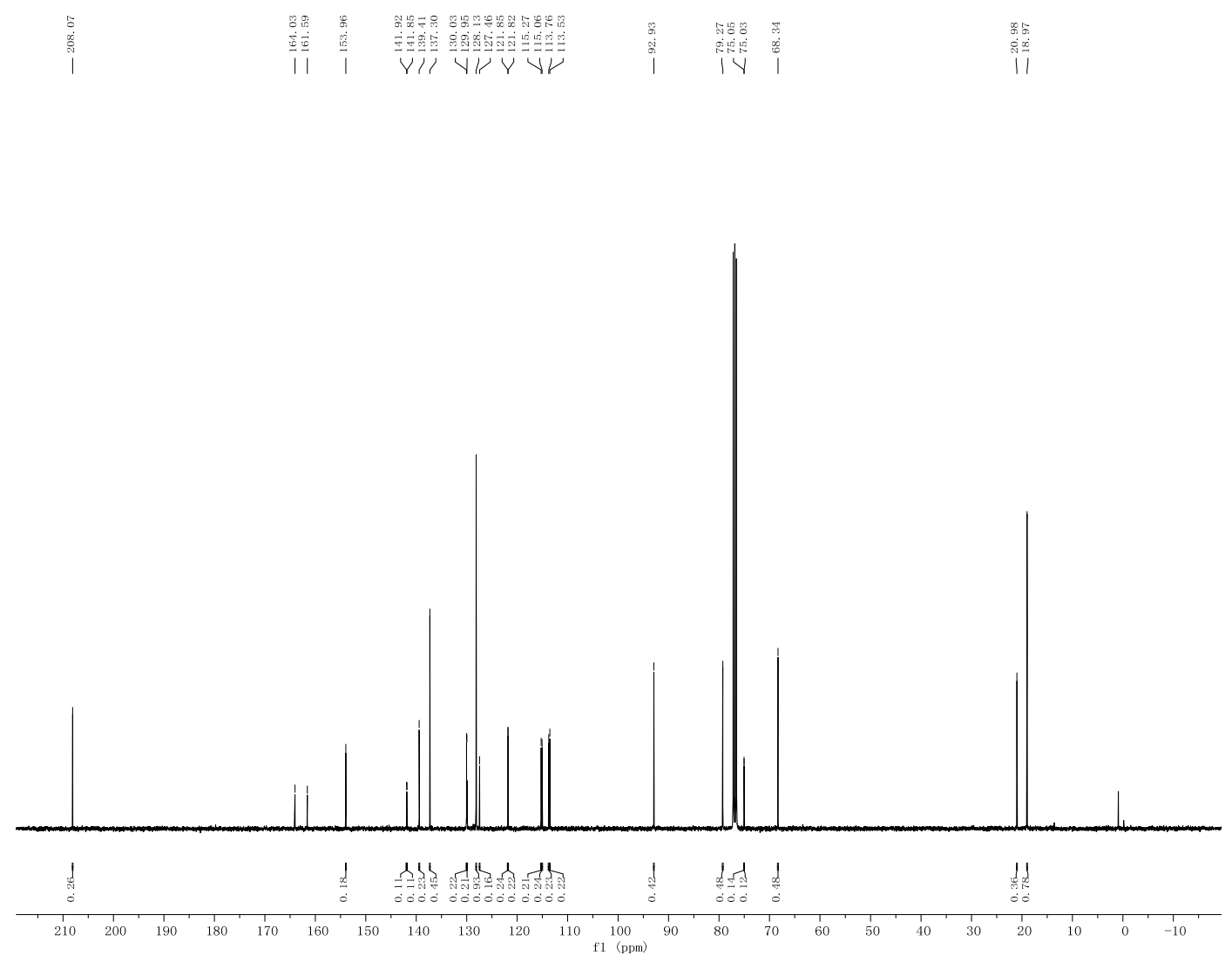
${ }^{1} \mathrm{H}$ NMR spectrum $\left(500 \mathrm{MHz}, \mathrm{CDCl}_{3}\right)$
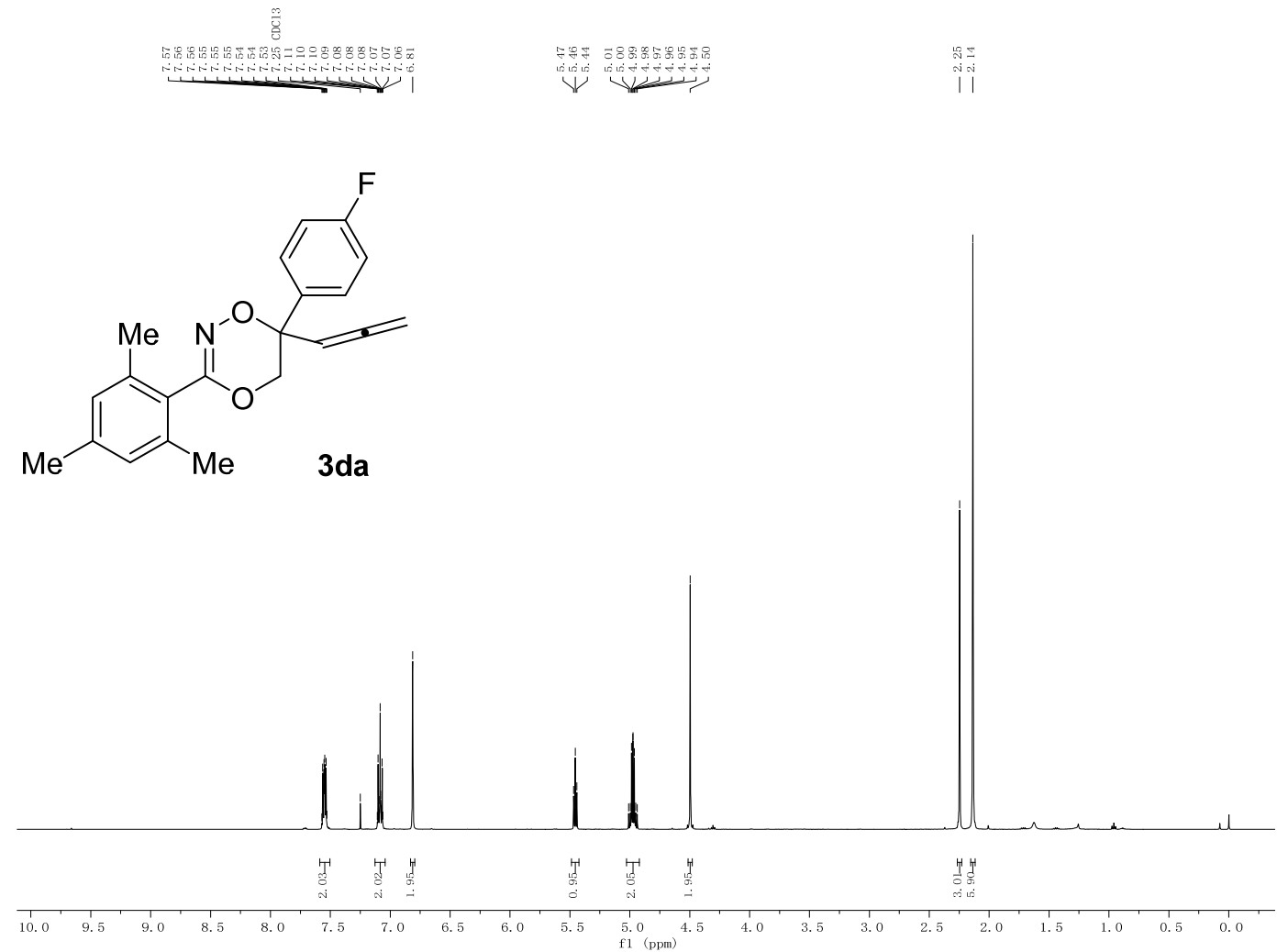

${ }^{13} \mathrm{C}$ NMR spectrum $\left(126 \mathrm{MHz}, \mathrm{CDCl}_{3}\right)$
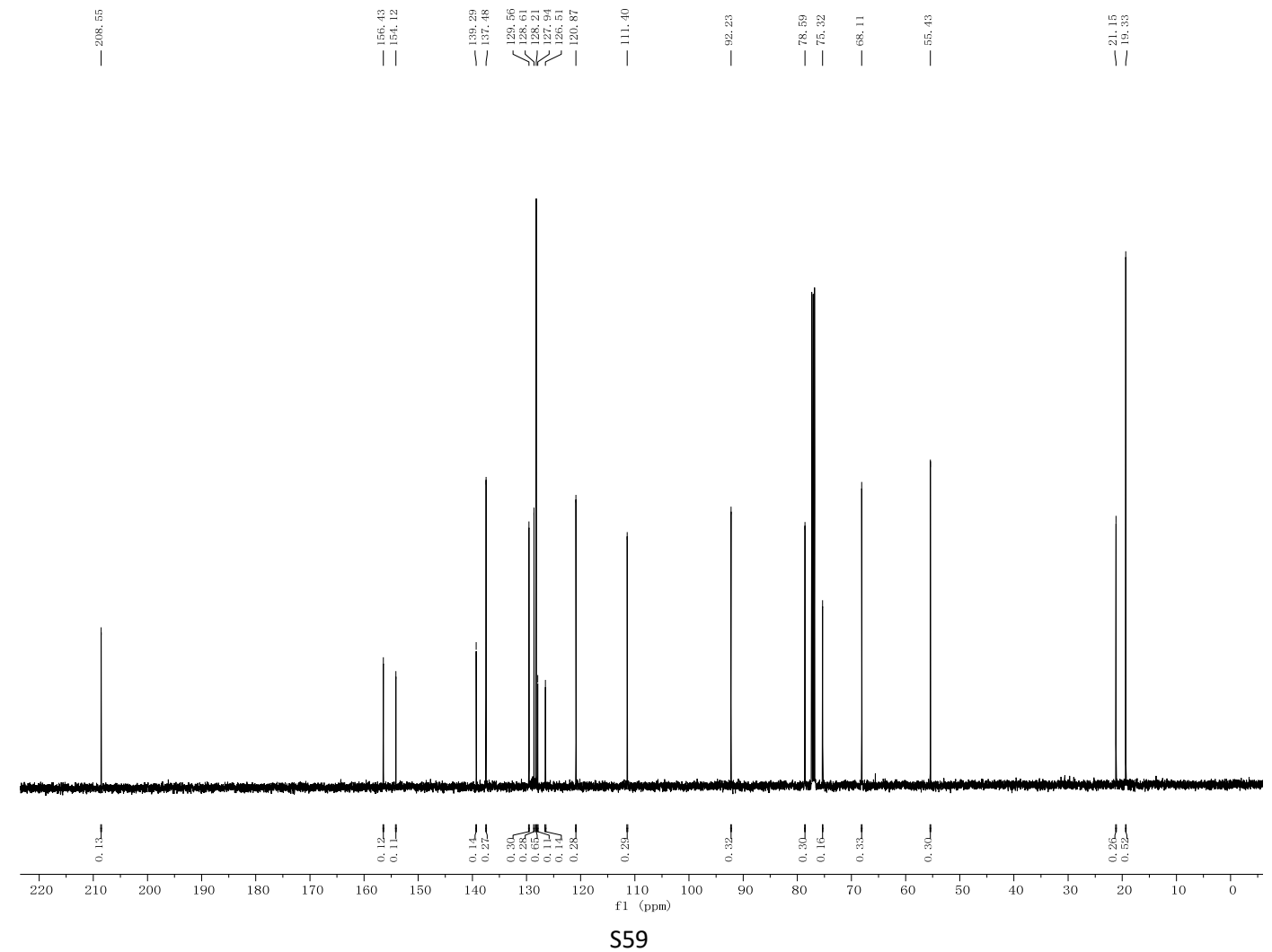
${ }^{1} \mathrm{H}$ NMR spectrum $\left(500 \mathrm{MHz}, \mathrm{CDCl}_{3}\right)$

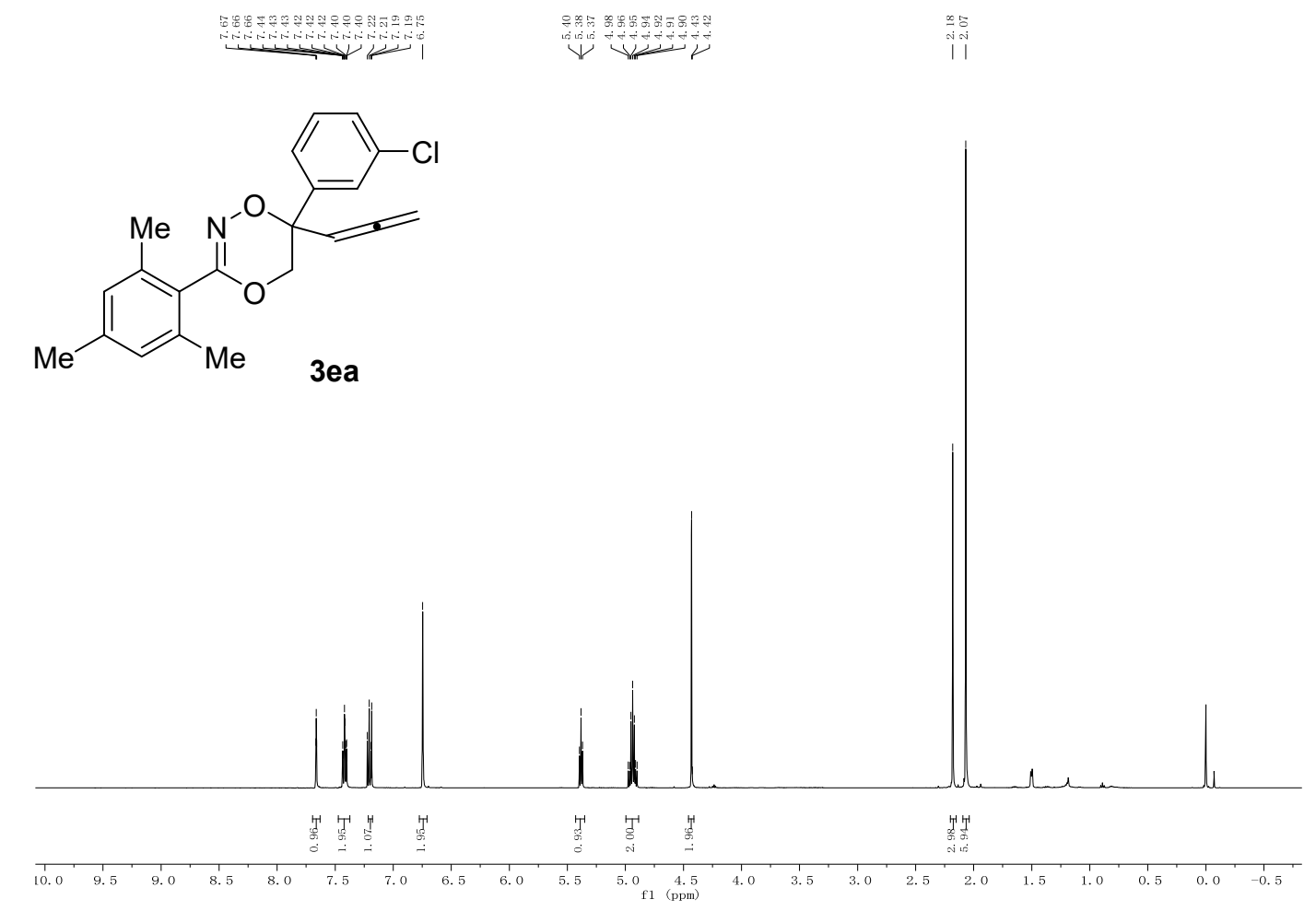

${ }^{13} \mathrm{C}$ NMR spectrum $\left(126 \mathrm{MHz}, \mathrm{CDCl}_{3}\right)$
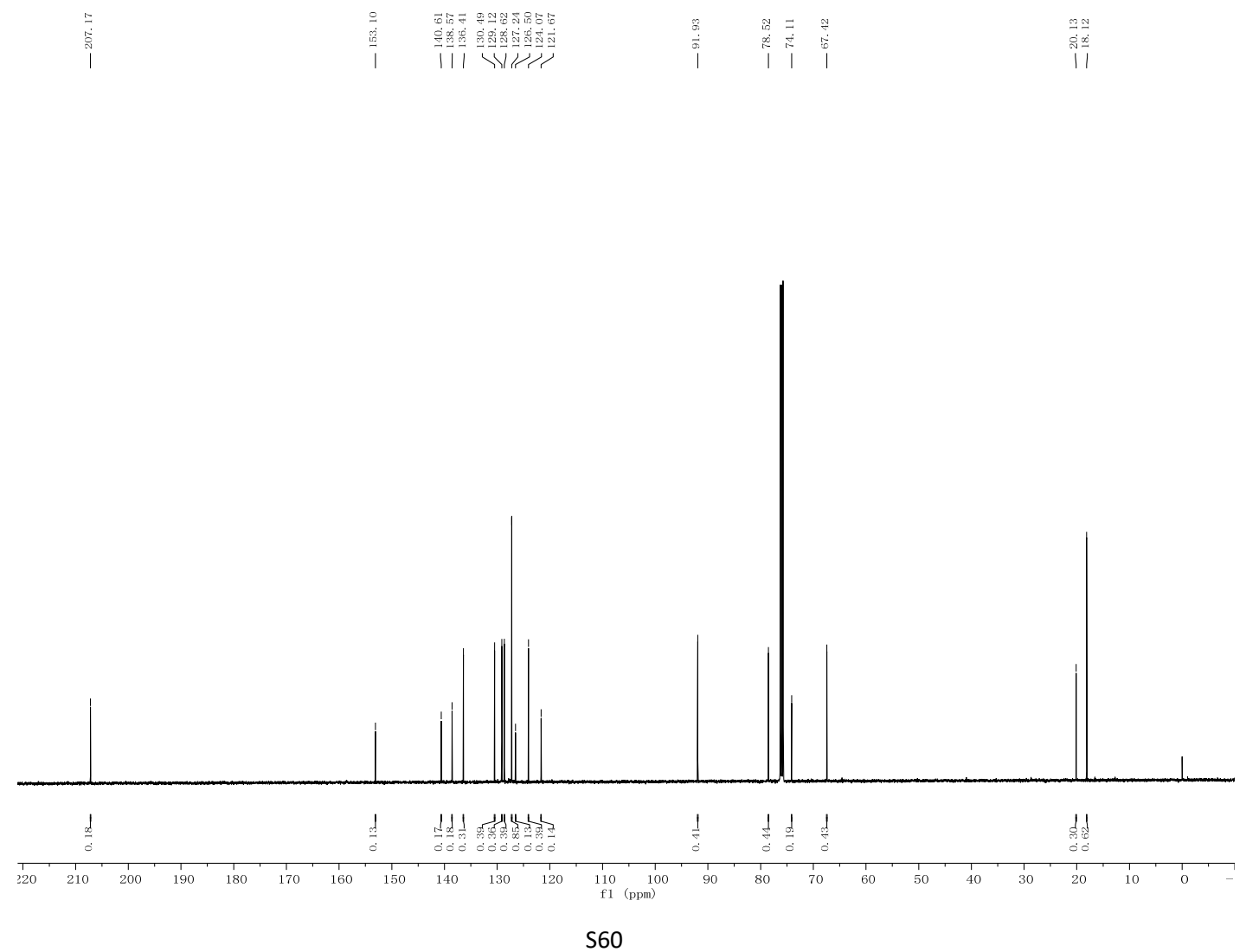


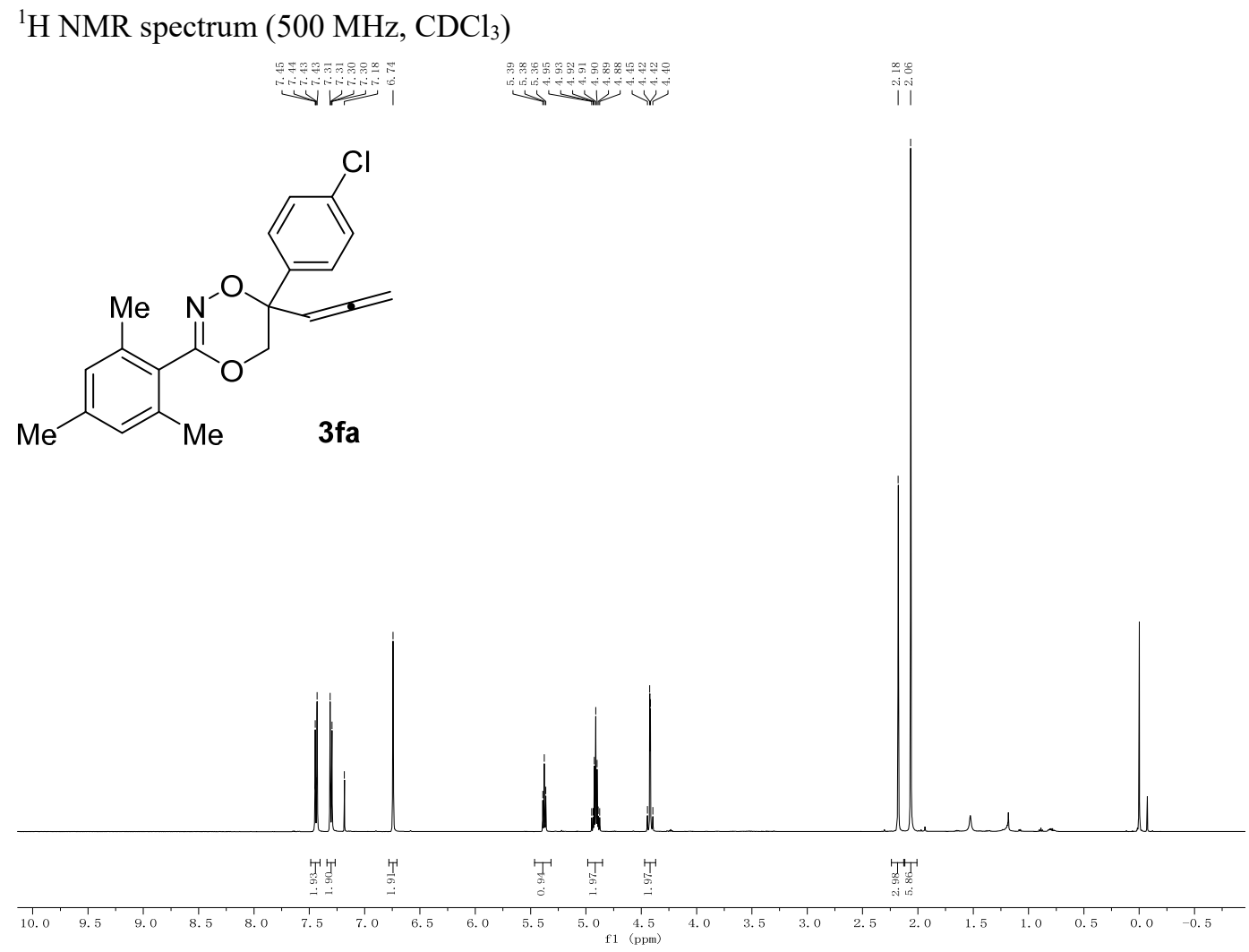

${ }^{13} \mathrm{C}$ NMR spectrum (126 MHz, $\left.\mathrm{CDCl}_{3}\right)$

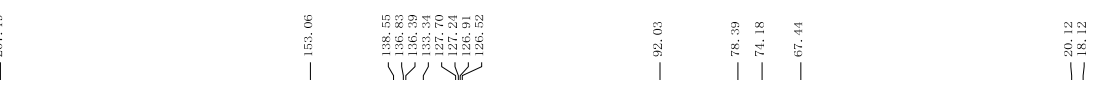
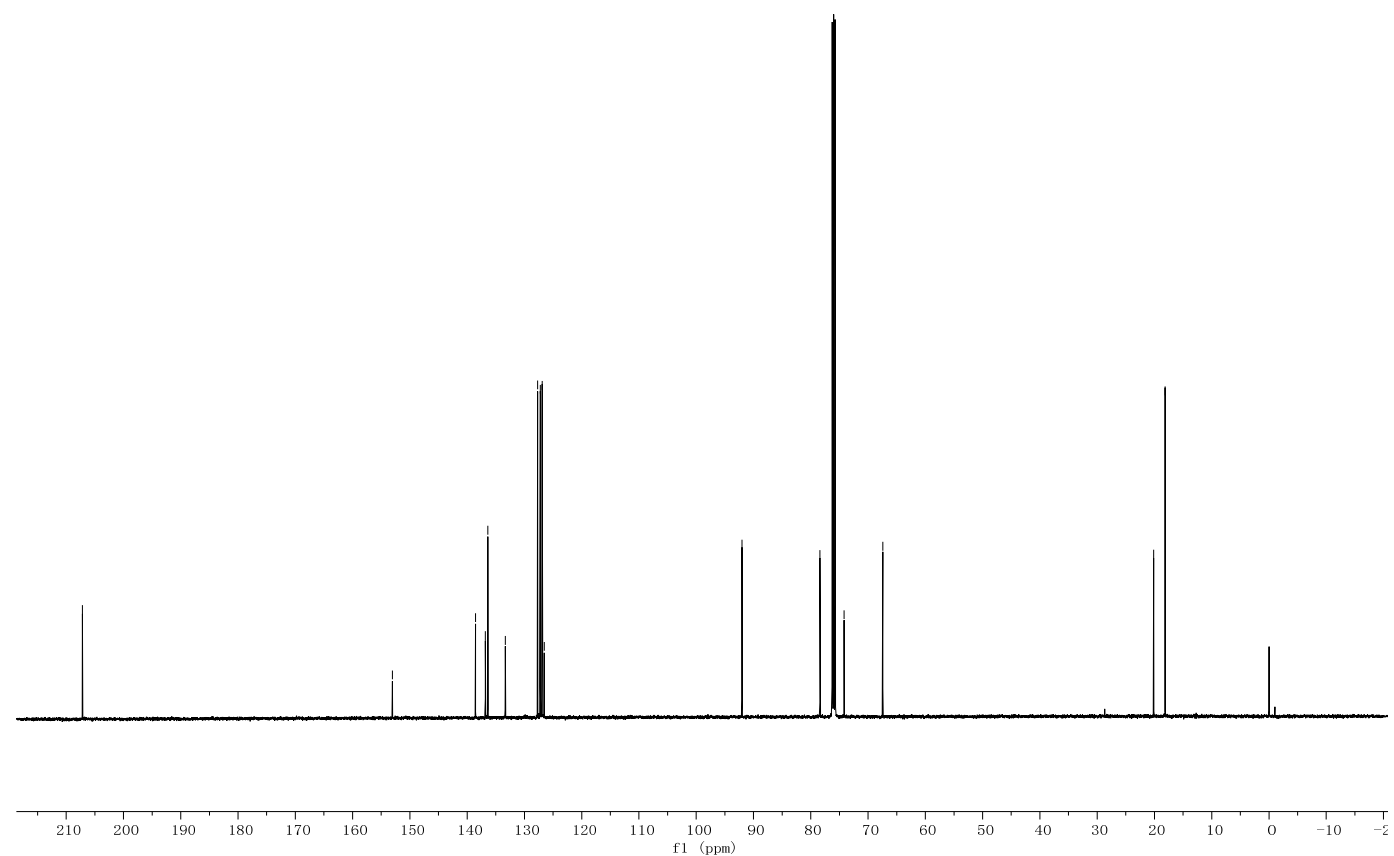
${ }^{1} \mathrm{H}$ NMR spectrum $\left(500 \mathrm{MHz}, \mathrm{CDCl}_{3}\right)$

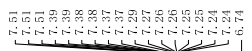

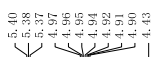
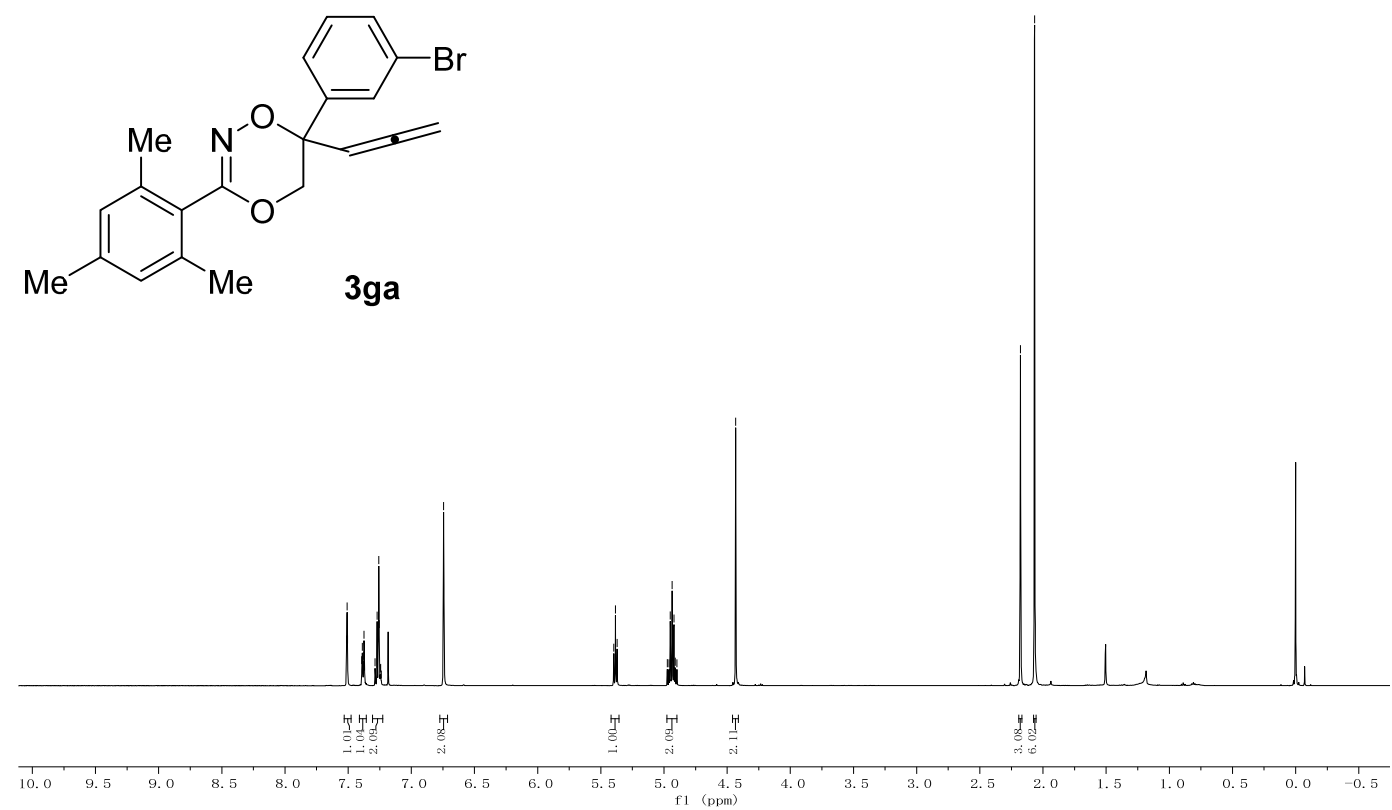

${ }^{13} \mathrm{C}$ NMR spectrum $\left(126 \mathrm{MHz}, \mathrm{CDCl}_{3}\right)$

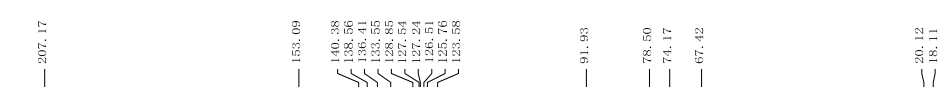

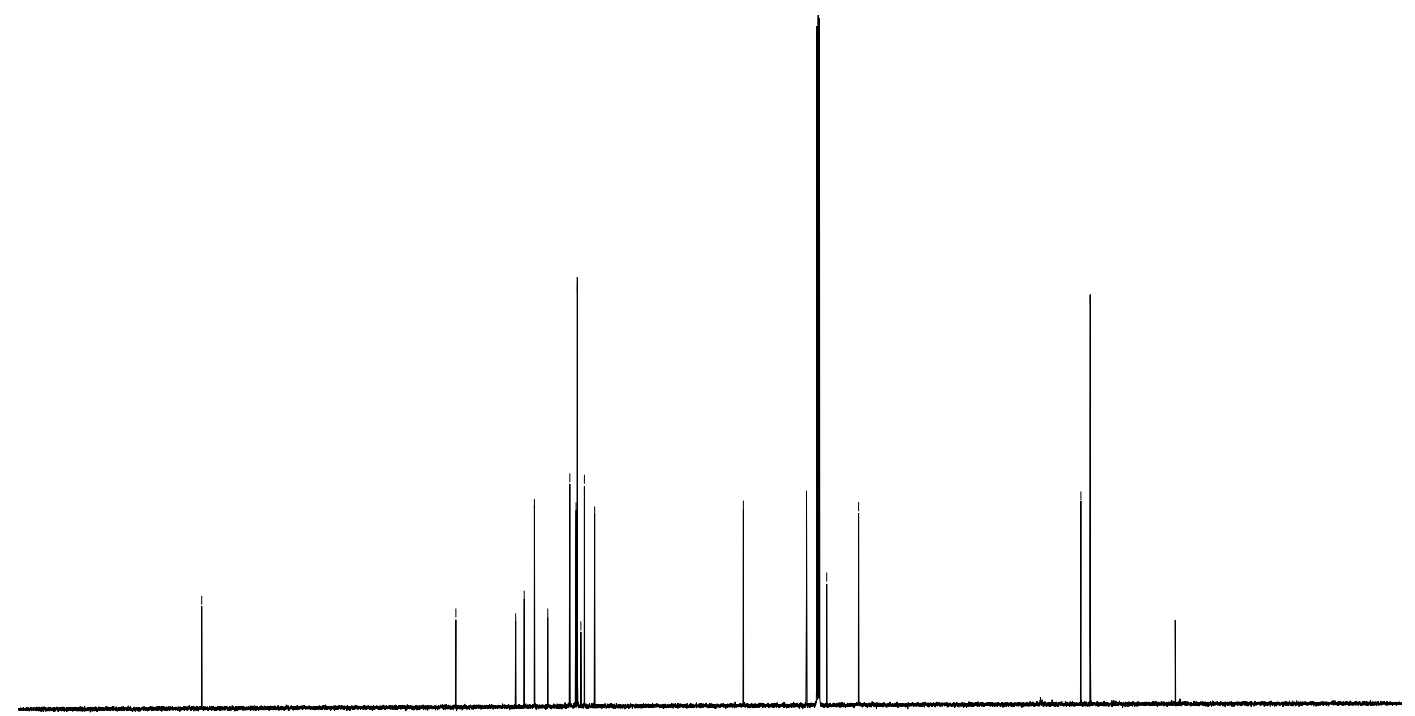

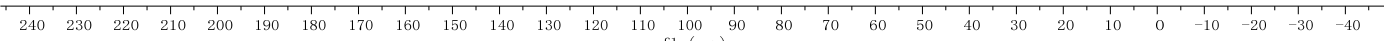




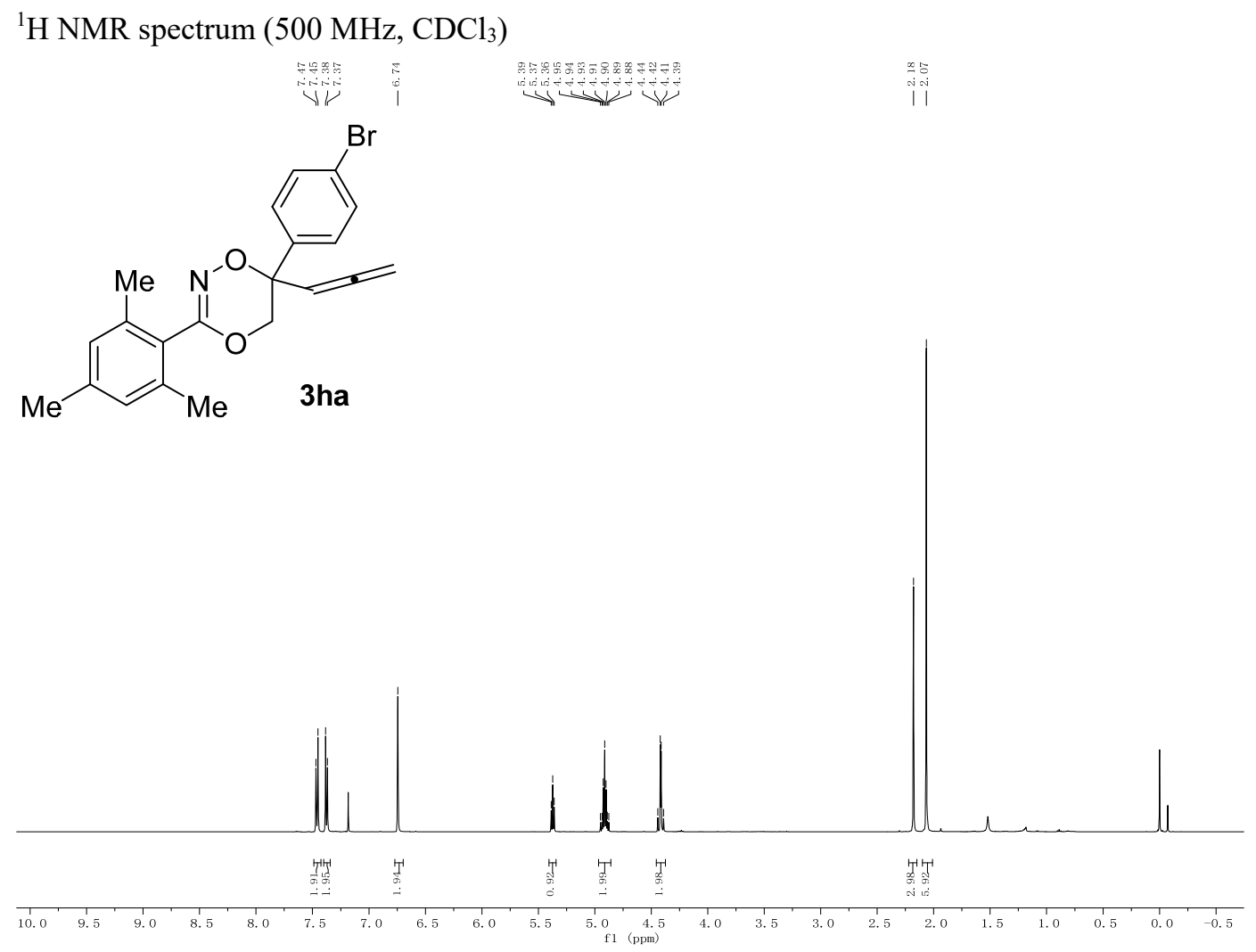

${ }^{13} \mathrm{C}$ NMR spectrum $\left(126 \mathrm{MHz}, \mathrm{CDCl}_{3}\right)$
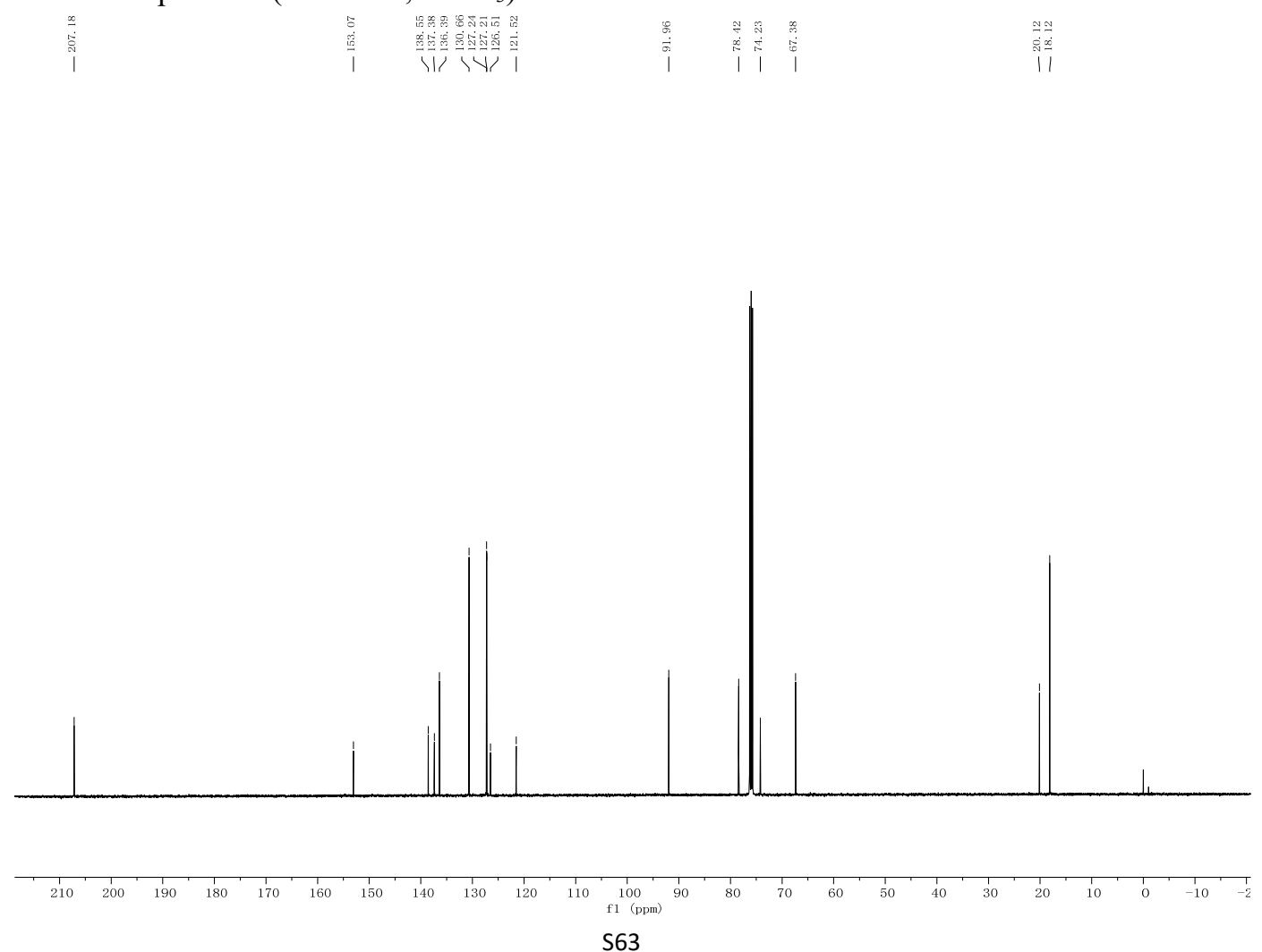
${ }^{1} \mathrm{H}$ NMR spectrum $\left(500 \mathrm{MHz}, \mathrm{CDCl}_{3}\right)$
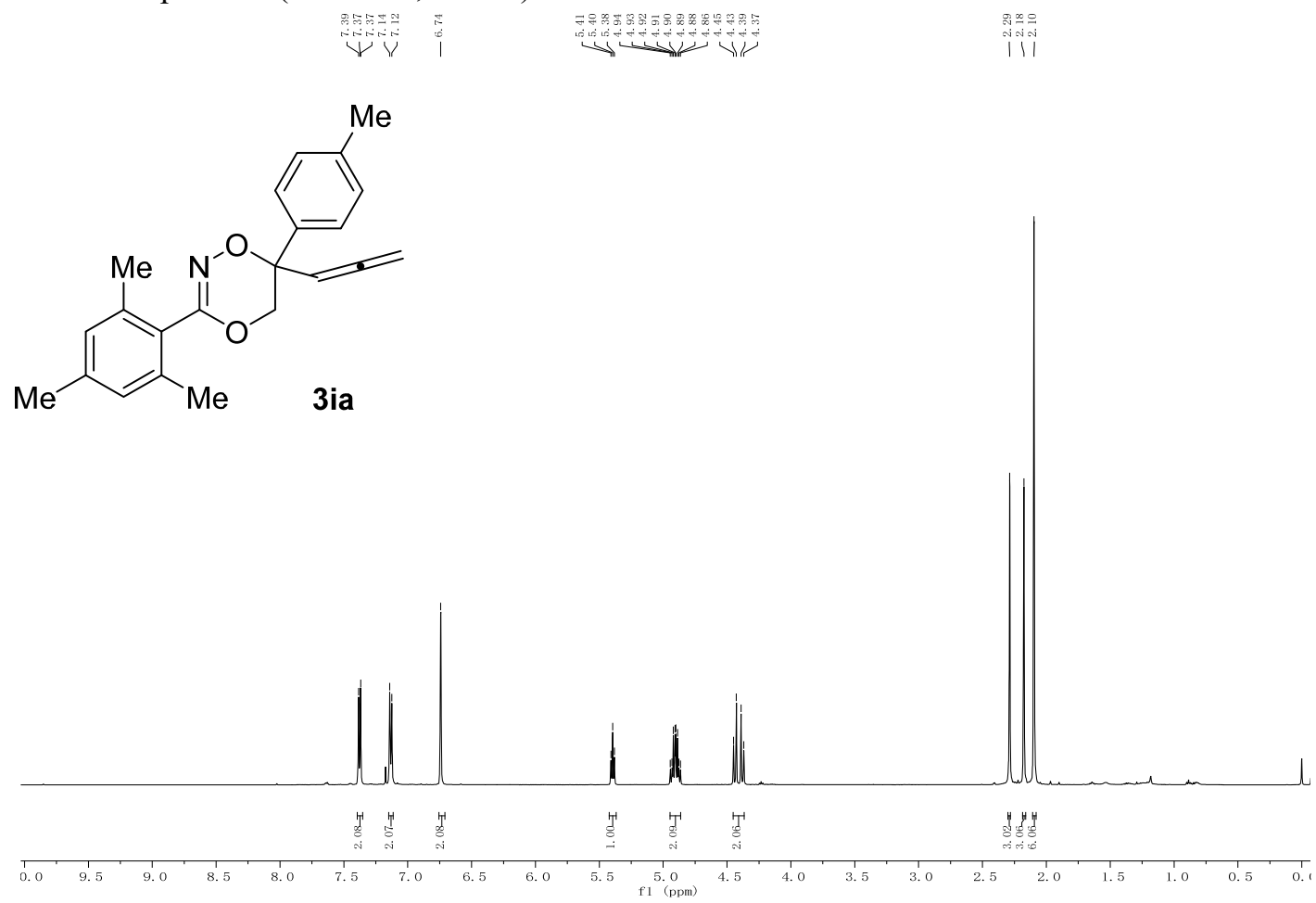

${ }^{13} \mathrm{C}$ NMR spectrum (101 MHz, $\mathrm{CDCl}_{3}$ )

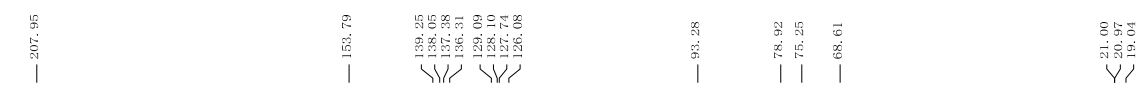
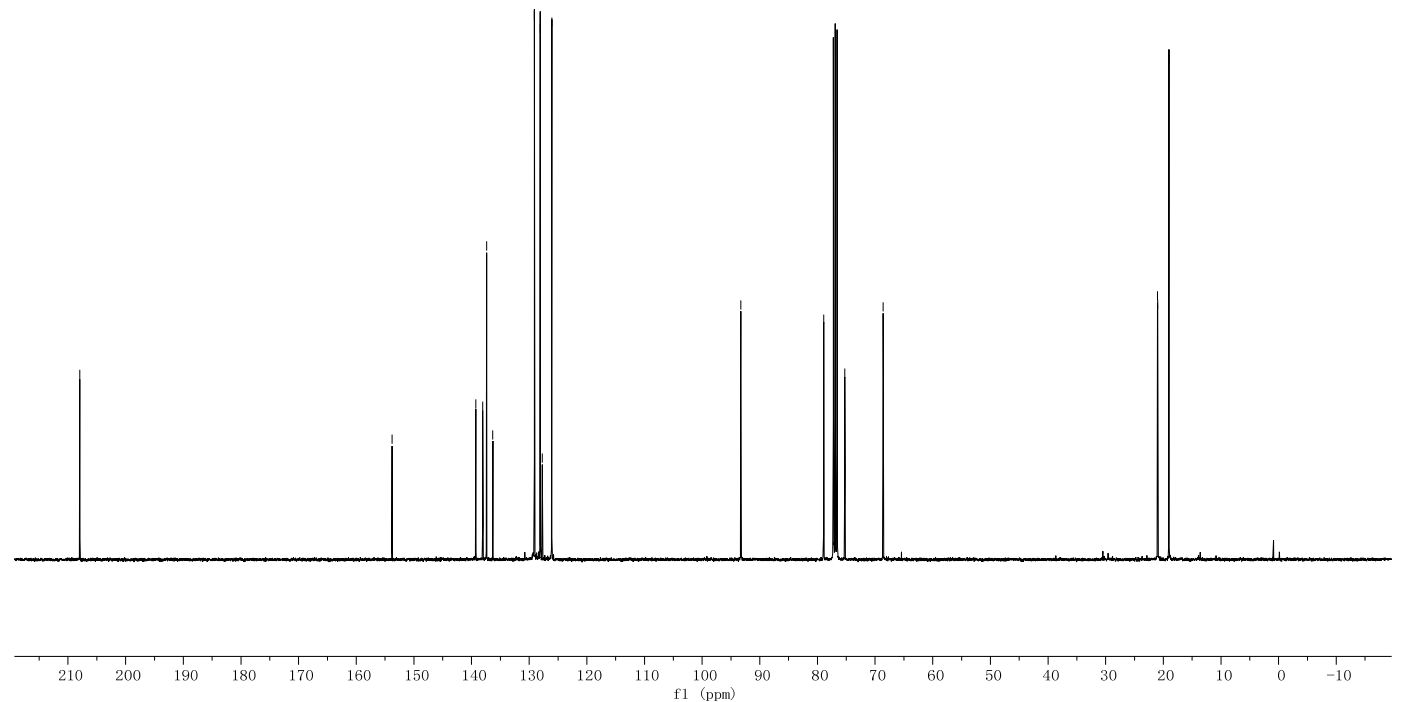
${ }^{1} \mathrm{H}$ NMR spectrum (500 MHz, $\mathrm{CDCl}_{3}$ )

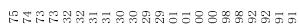
$\underbrace{8}$

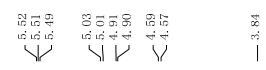

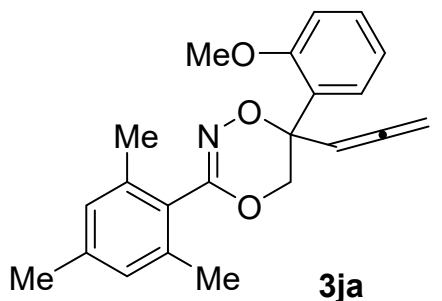

3ja

${ }^{13} \mathrm{C}$ NMR spectrum $\left(126 \mathrm{MHz}, \mathrm{CDCl}_{3}\right)$

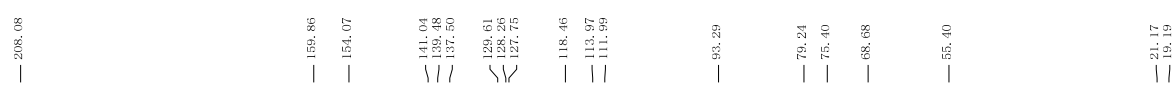
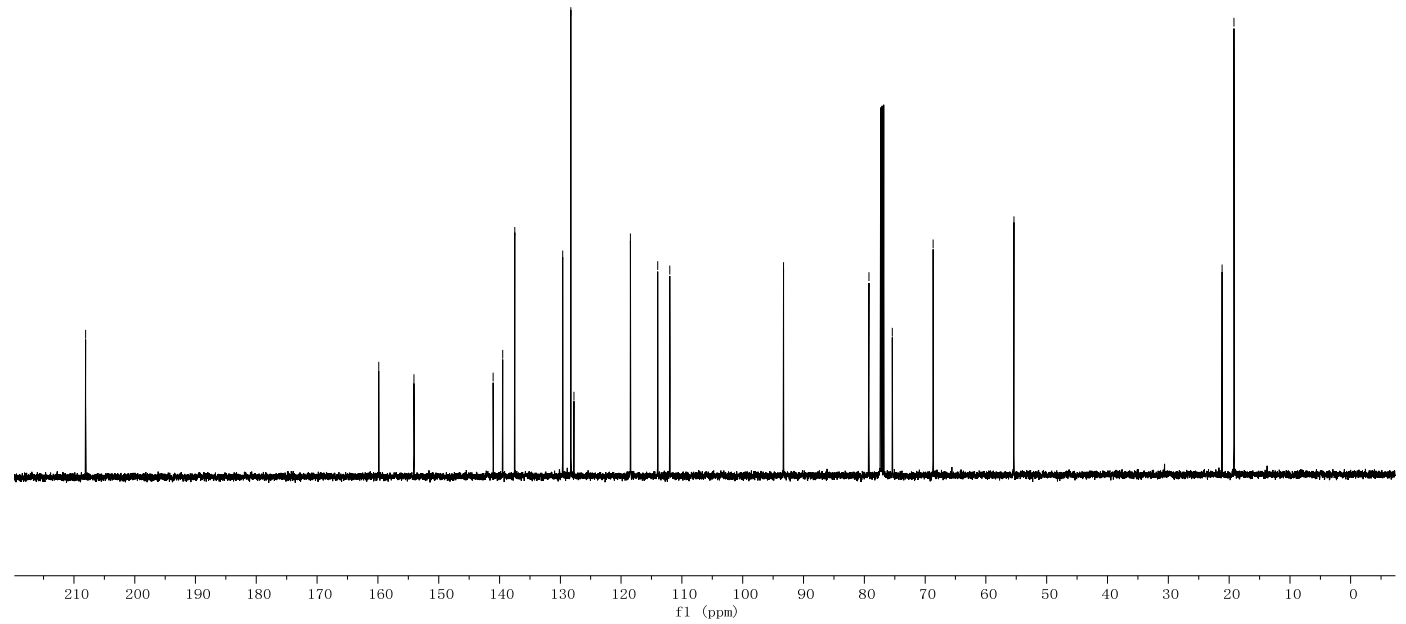
${ }^{1} \mathrm{H}$ NMR spectrum $\left(500 \mathrm{MHz}, \mathrm{CDCl}_{3}\right)$

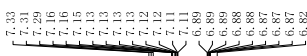
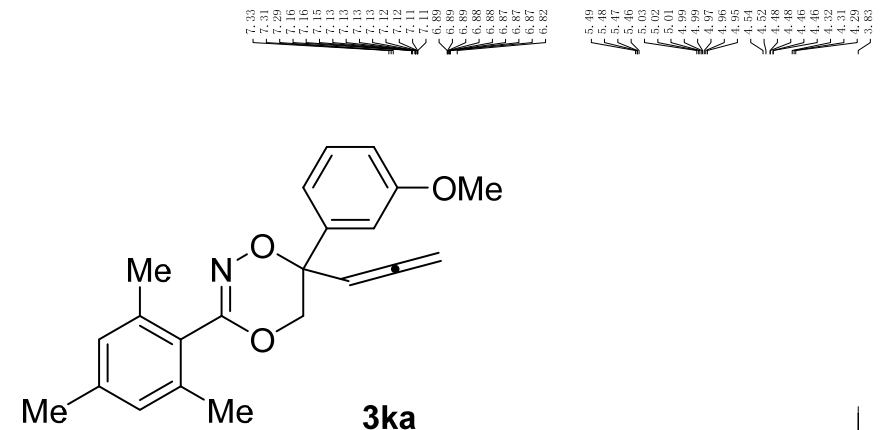

3ka
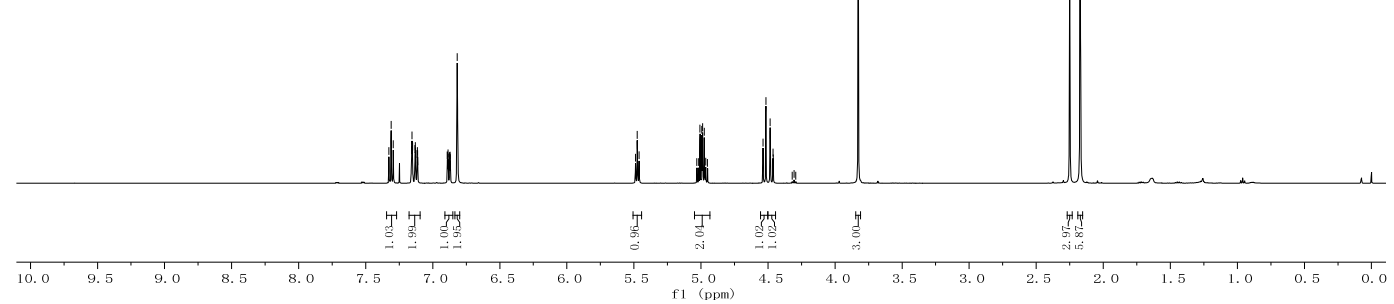

${ }^{13} \mathrm{C}$ NMR spectrum $\left(126 \mathrm{MHz}, \mathrm{CDCl}_{3}\right)$

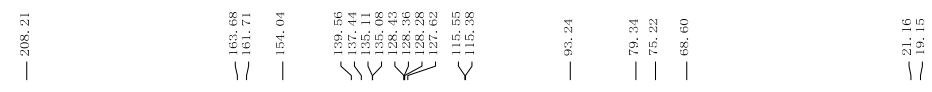
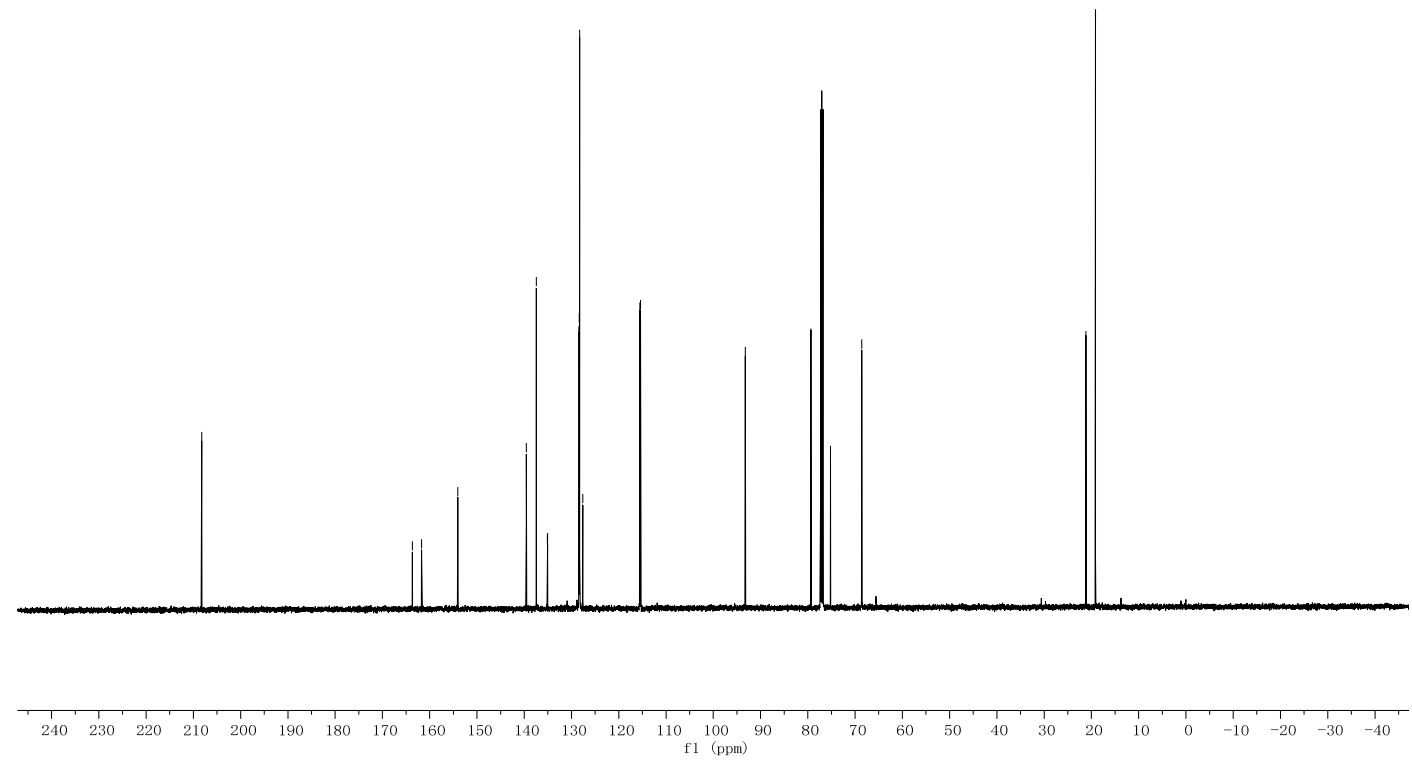
${ }^{1} \mathrm{H}$ NMR spectrum $\left(500 \mathrm{MHz}, \mathrm{CDCl}_{3}\right)$

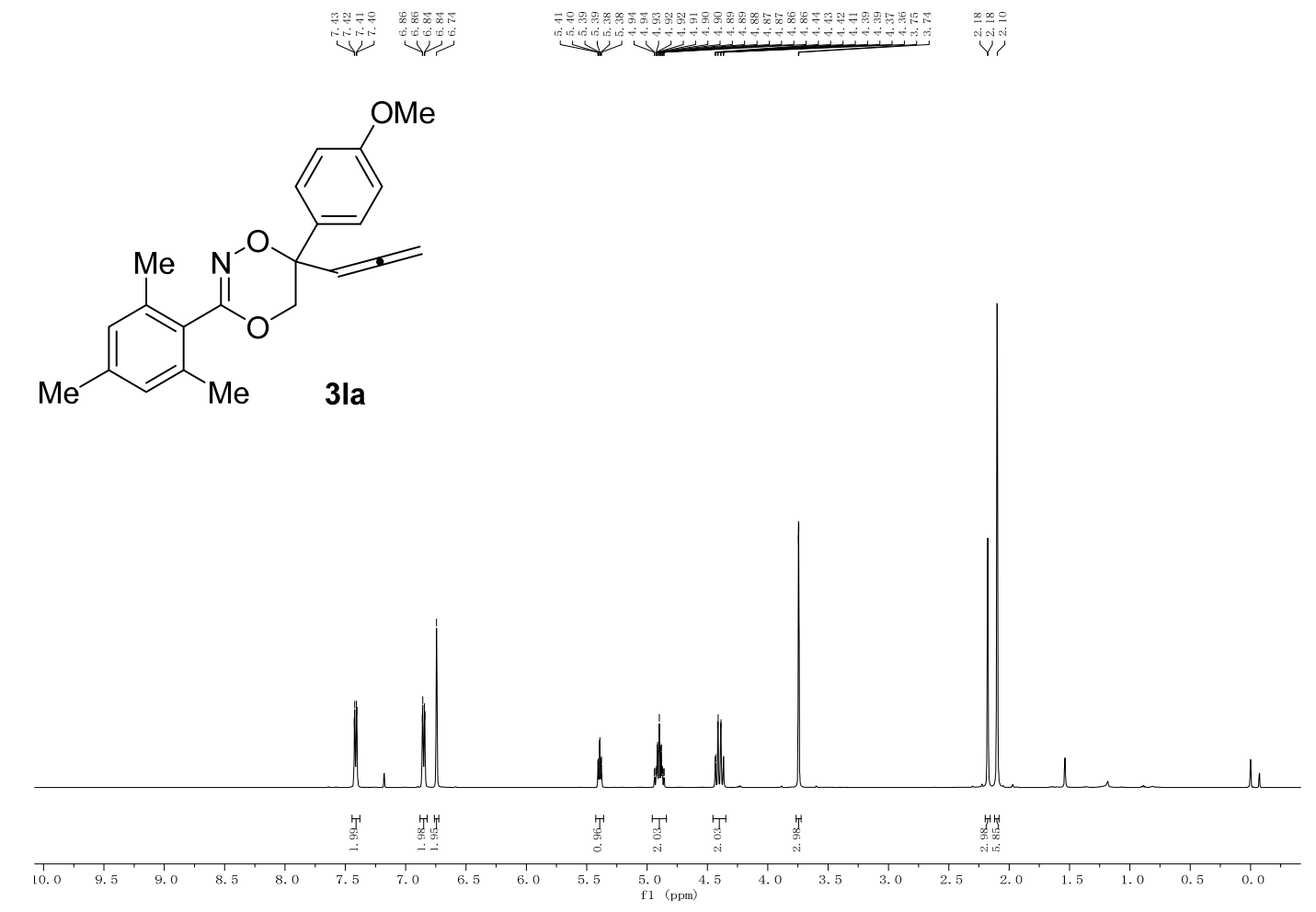

${ }^{13} \mathrm{C}$ NMR spectrum $\left(126 \mathrm{MHz}, \mathrm{CDCl}_{3}\right)$

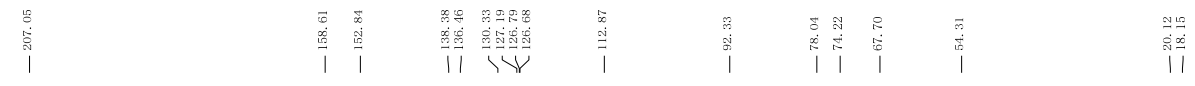

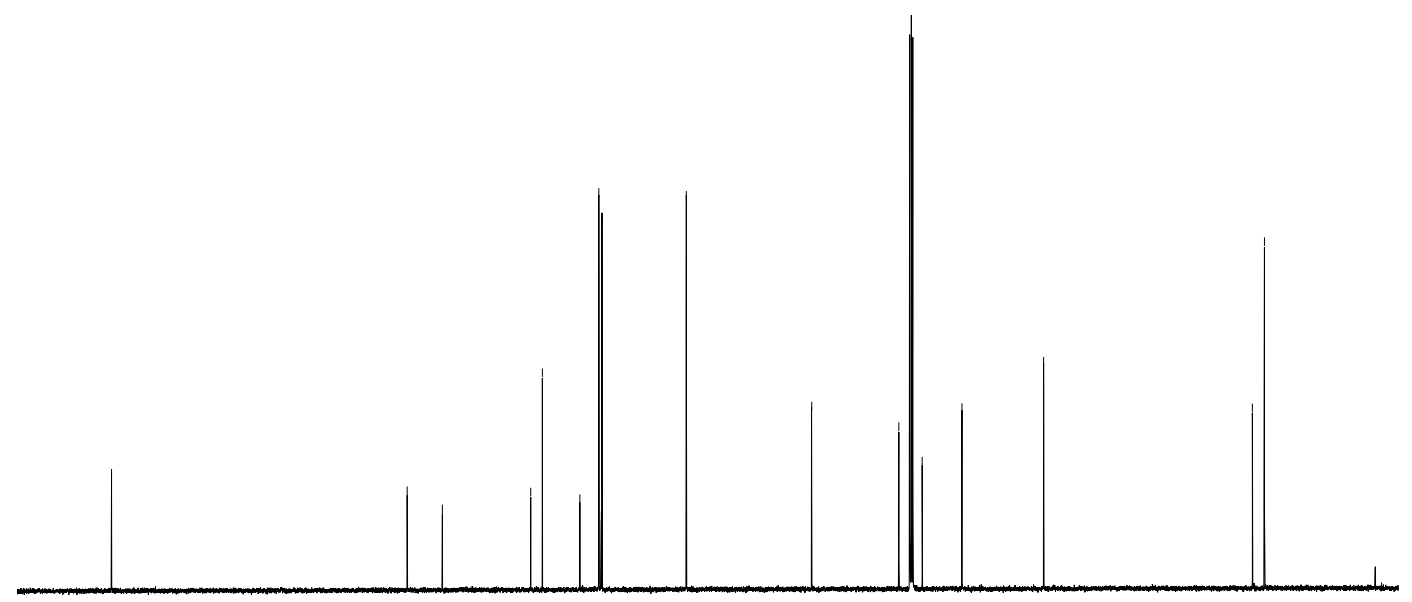

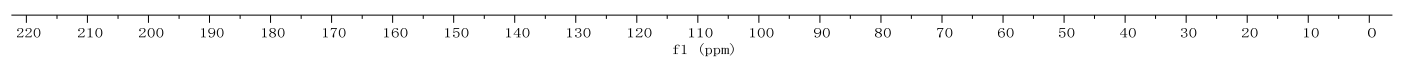


${ }^{1} \mathrm{H}$ NMR spectrum (500 MHz, $\mathrm{CDCl}_{3}$ )

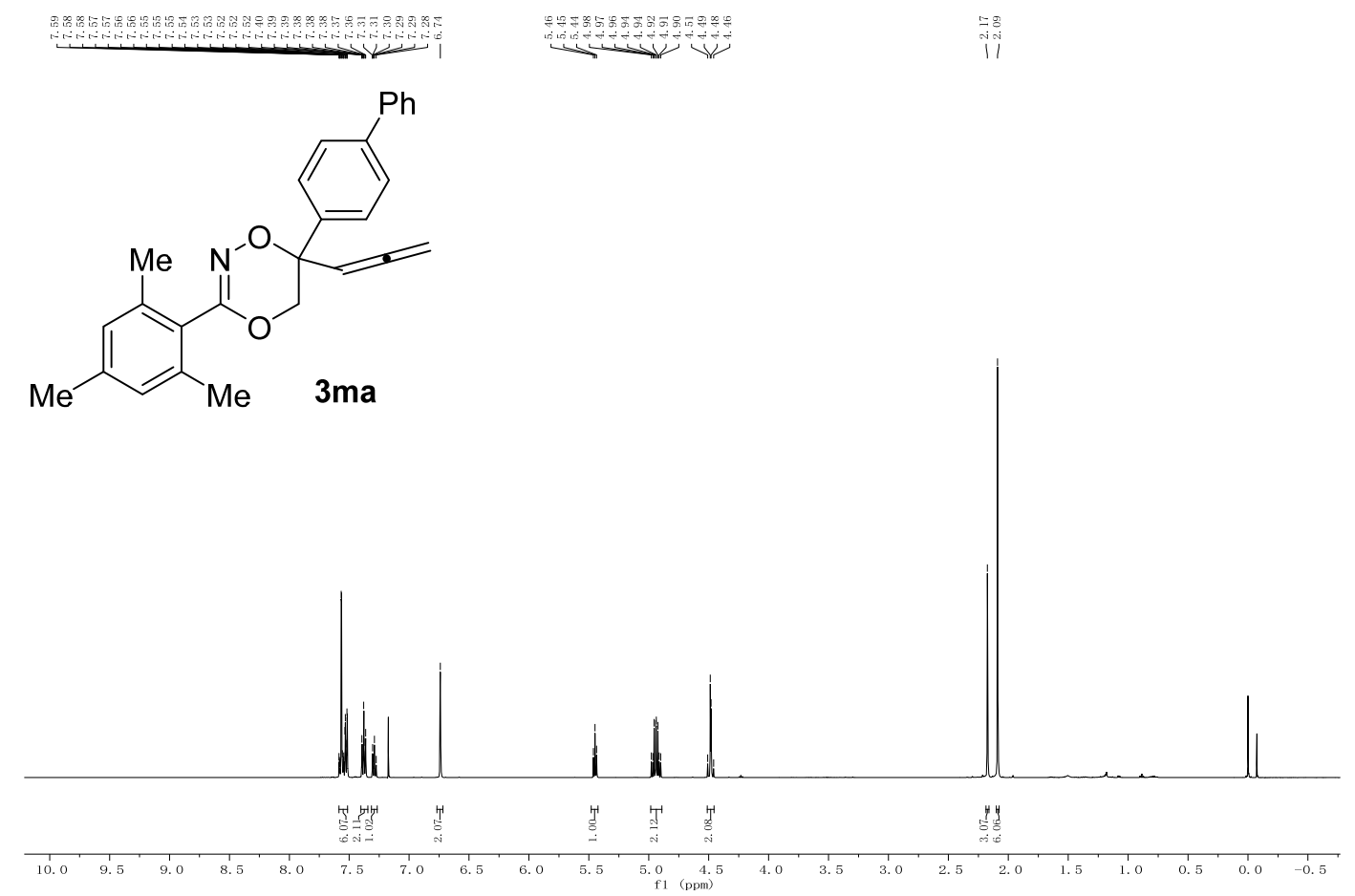

${ }^{13} \mathrm{C}$ NMR spectrum (101 MHz, $\left.\mathrm{CDCl}_{3}\right)$

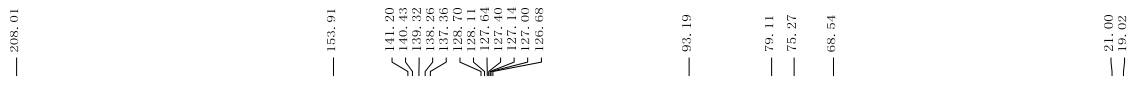
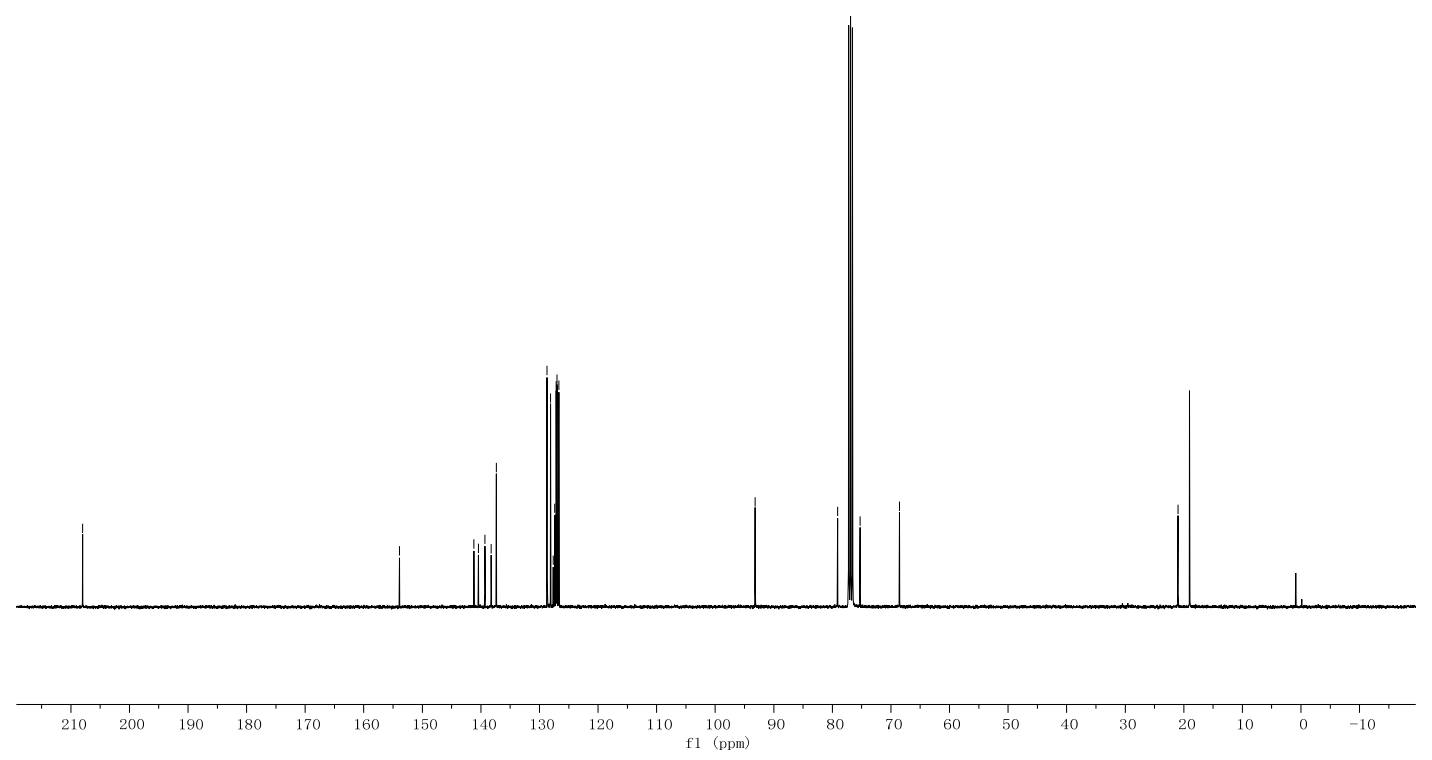
${ }^{1} \mathrm{H}$ NMR spectrum (500 MHz, $\mathrm{CDCl}_{3}$ )
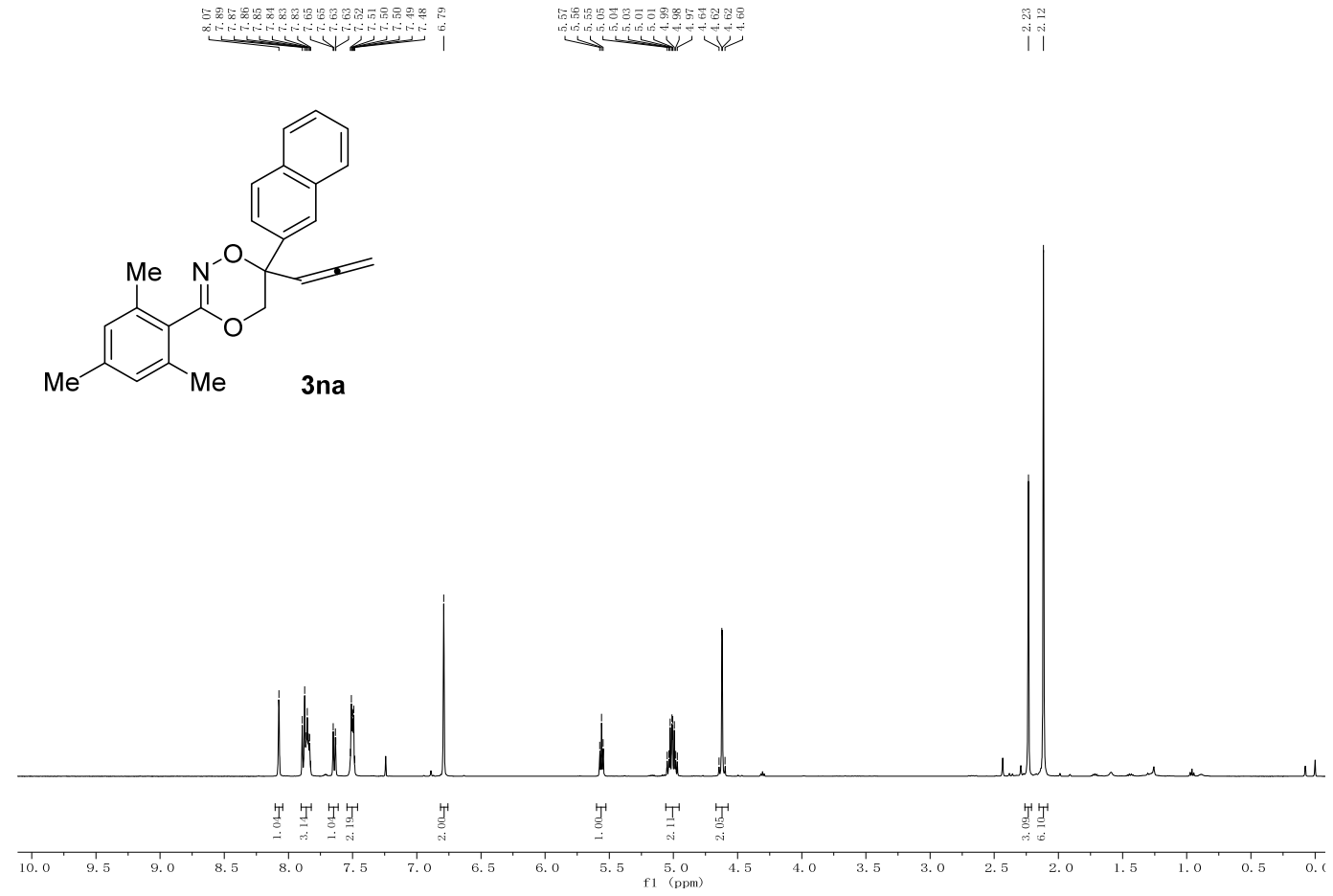

${ }^{13} \mathrm{C}$ NMR spectrum $\left(101 \mathrm{MHz}, \mathrm{CDCl}_{3}\right)$

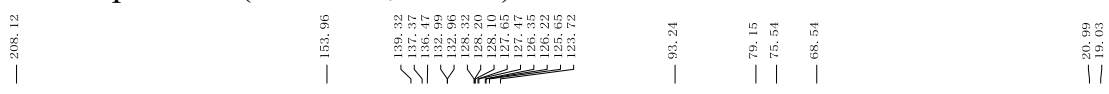
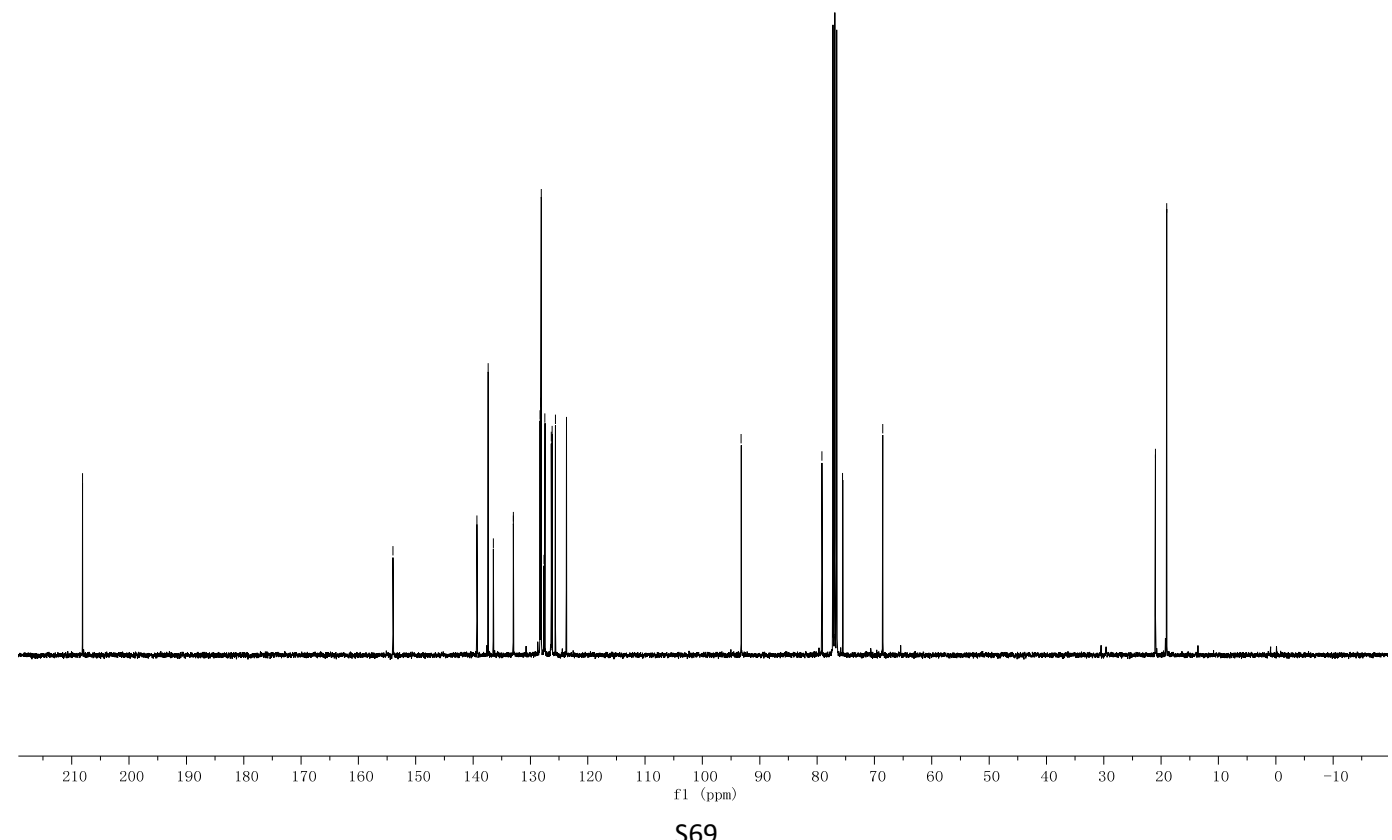
${ }^{1} \mathrm{H}$ NMR spectrum $\left(500 \mathrm{MHz}, \mathrm{CDCl}_{3}\right)$

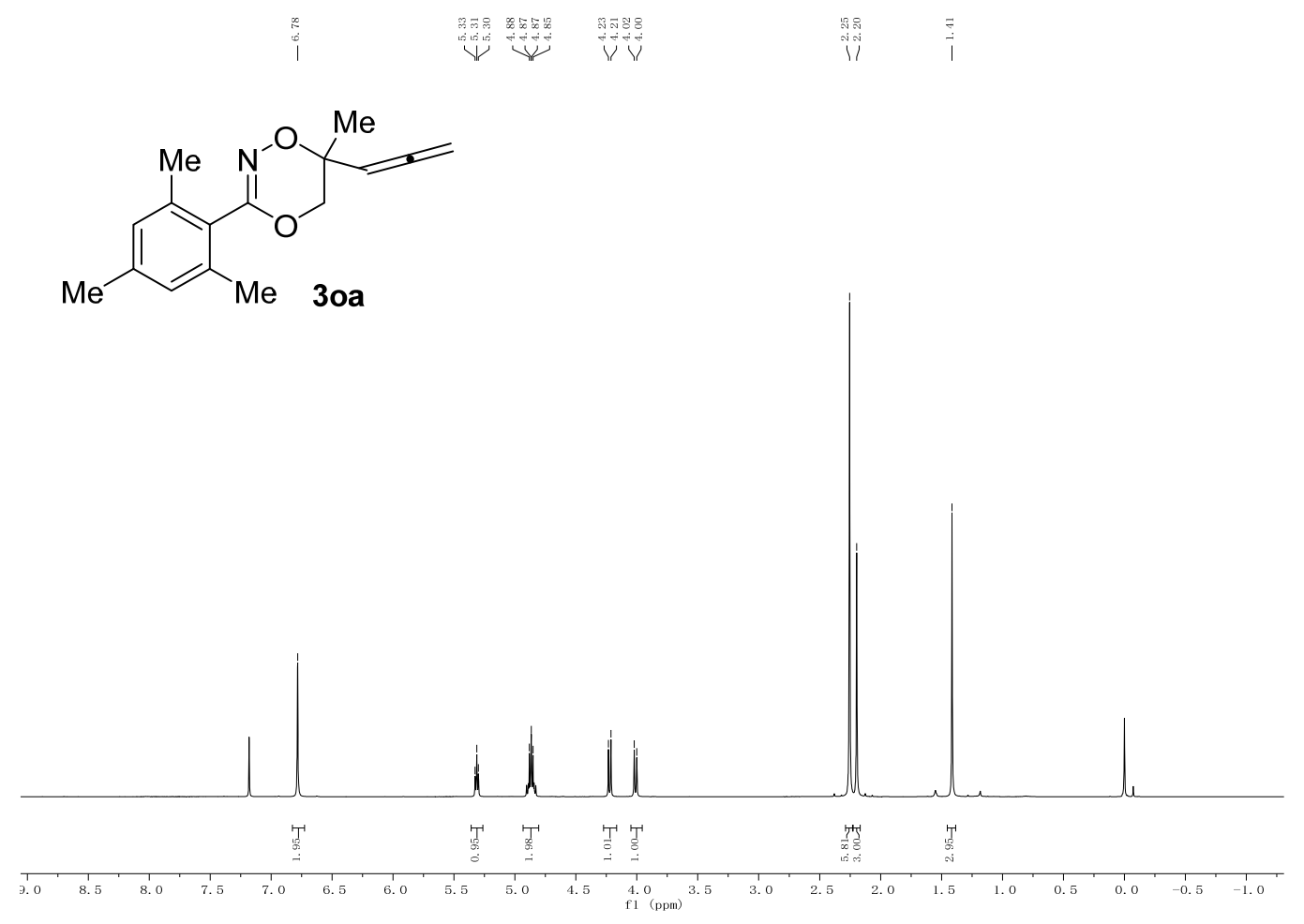

${ }^{13} \mathrm{C}$ NMR spectrum $\left(126 \mathrm{MHz}, \mathrm{CDCl}_{3}\right)$

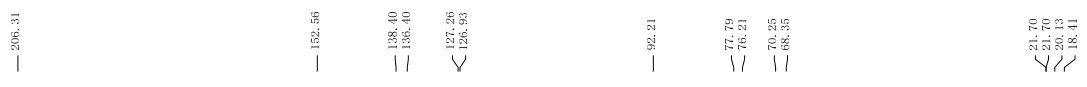
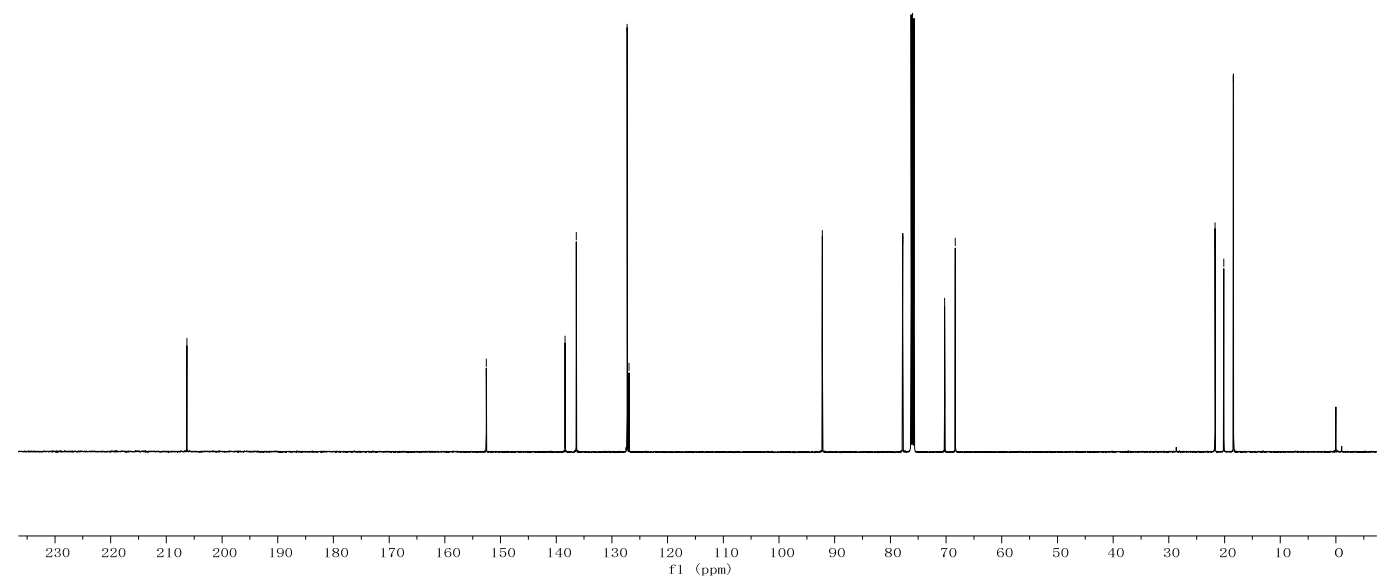
${ }^{1} \mathrm{H}$ NMR spectrum $\left(500 \mathrm{MHz}, \mathrm{CDCl}_{3}\right)$

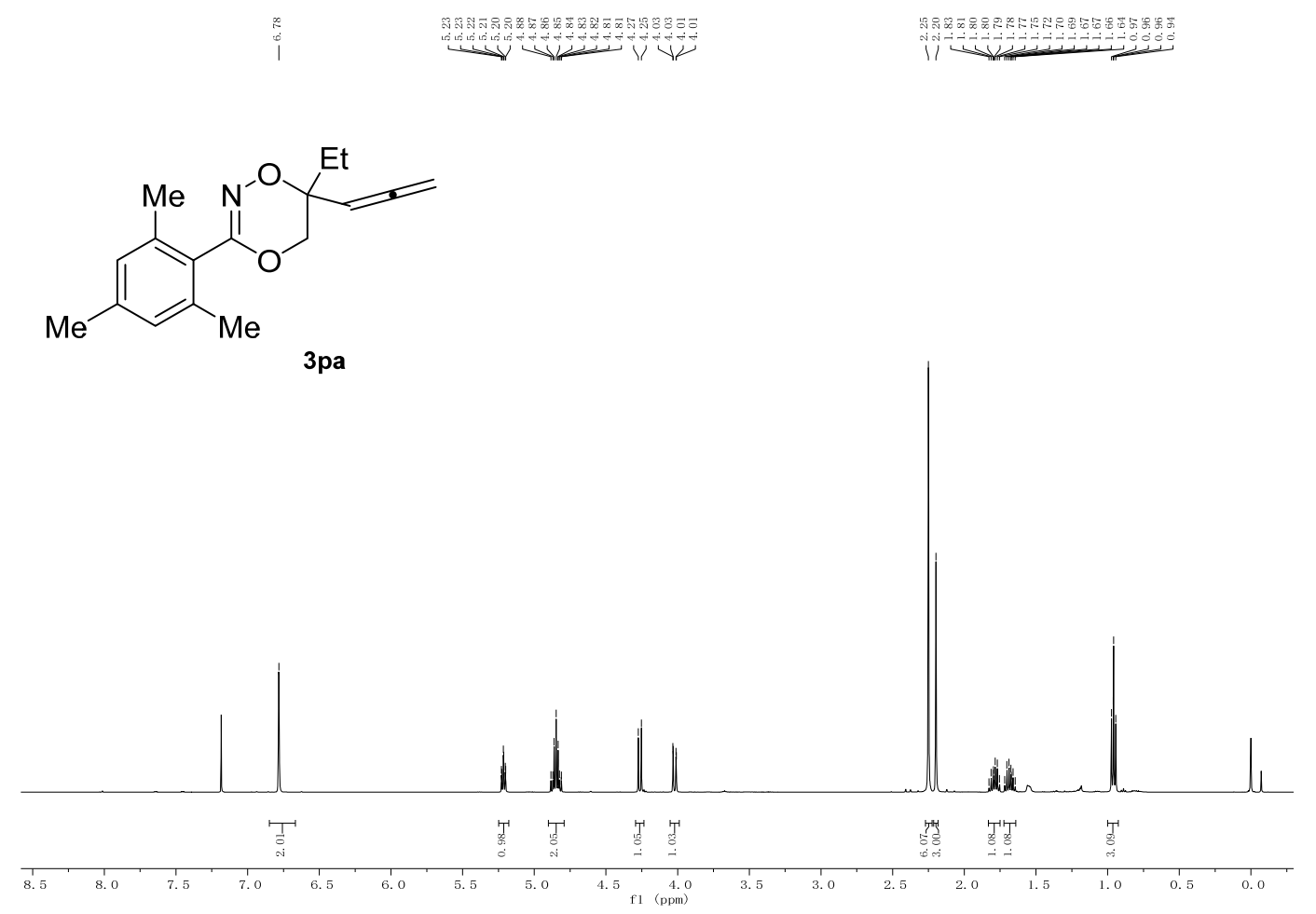

${ }^{13} \mathrm{C}$ NMR spectrum $\left(126 \mathrm{MHz}, \mathrm{CDCl}_{3}\right)$
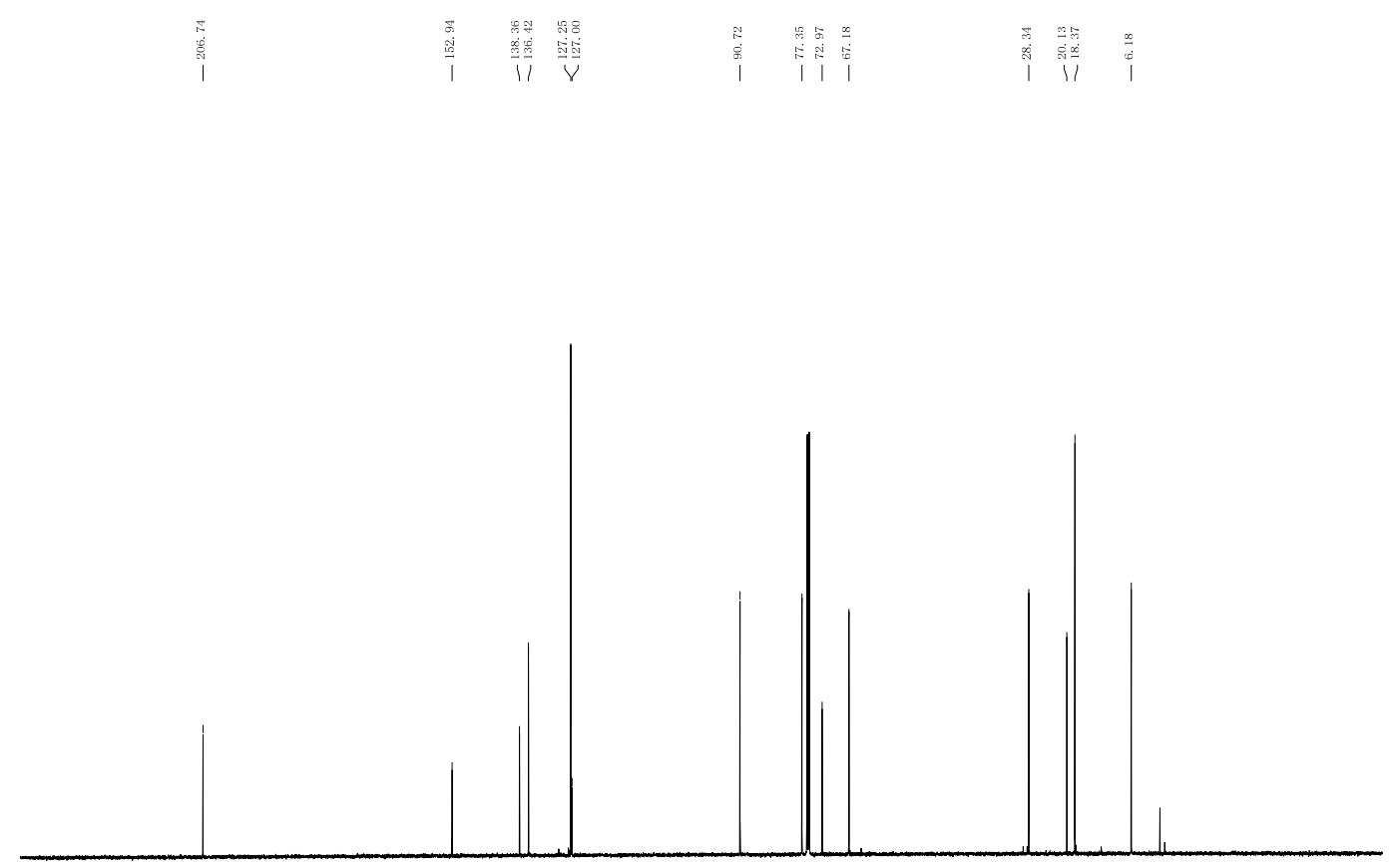

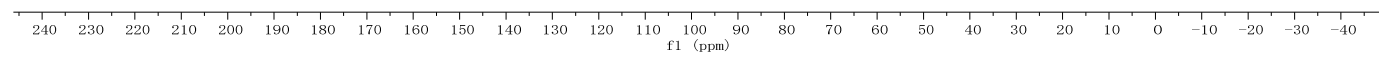


${ }^{1} \mathrm{H}$ NMR spectrum $\left(500 \mathrm{MHz}, \mathrm{CDCl}_{3}\right)$

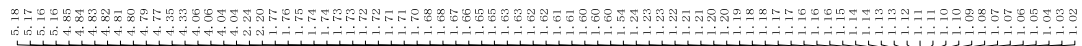
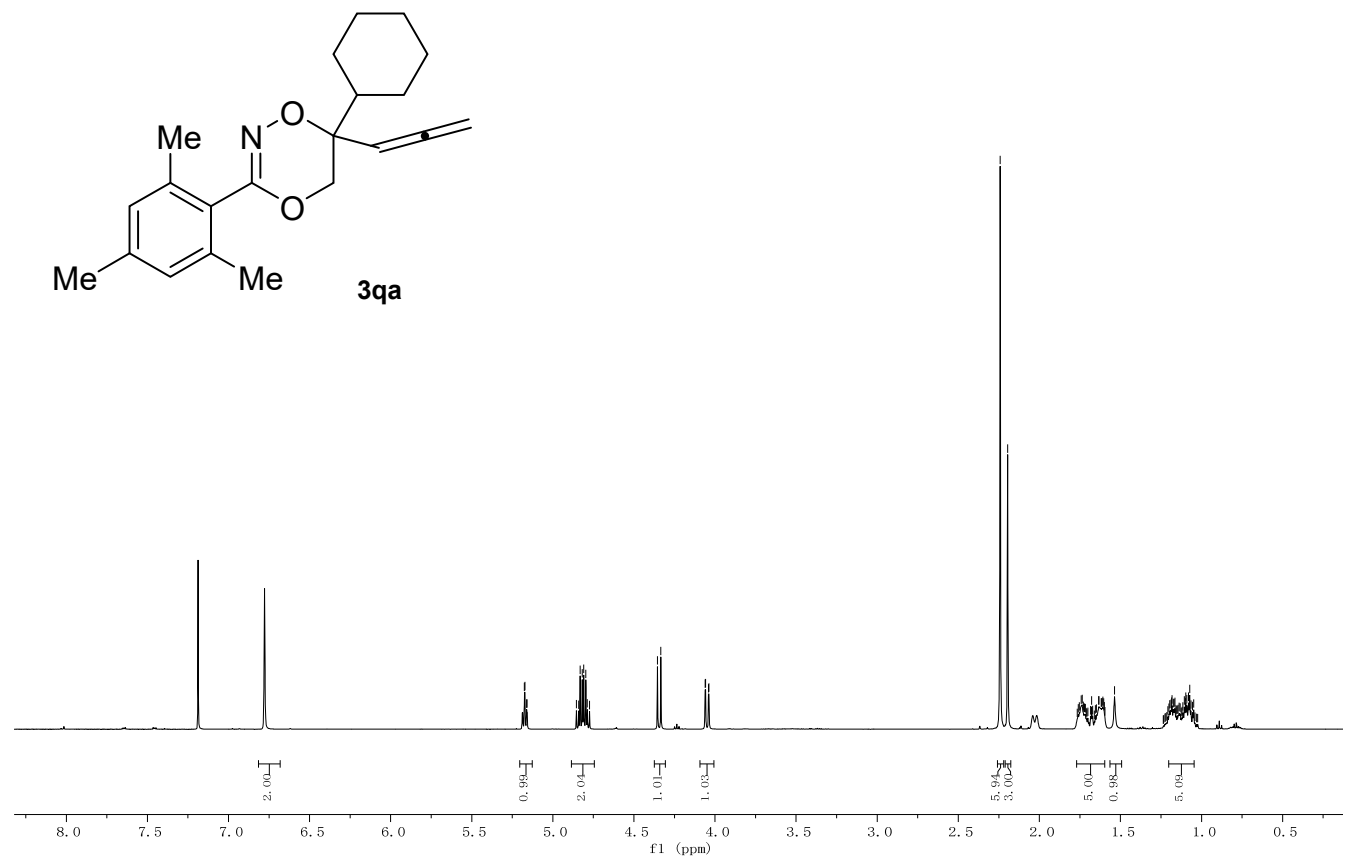

${ }^{13} \mathrm{C}$ NMR spectrum $\left(126 \mathrm{MHz}, \mathrm{CDCl}_{3}\right)$
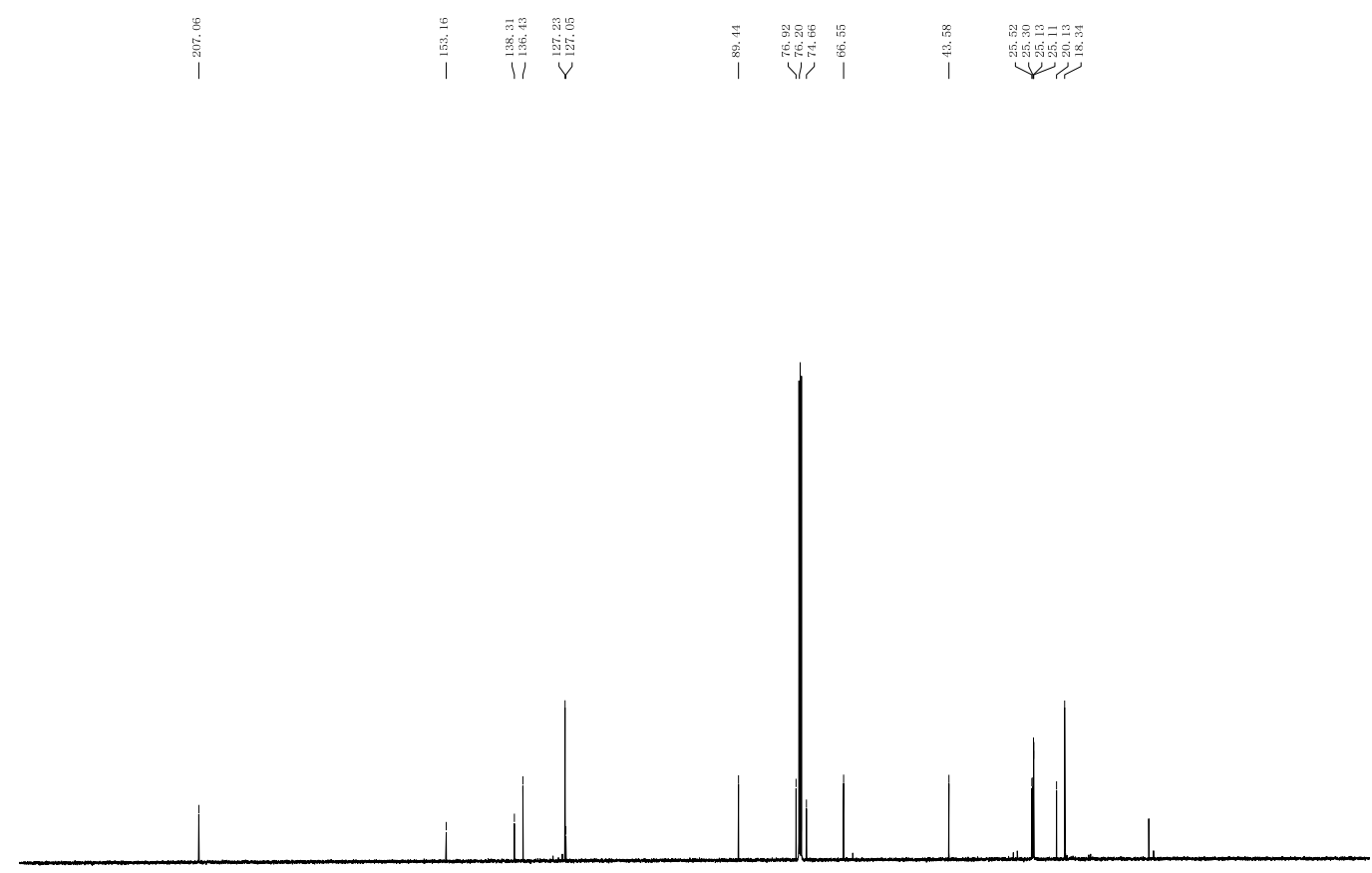

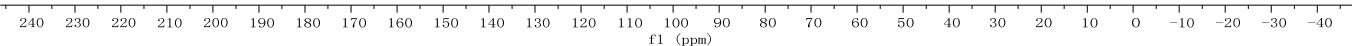


${ }^{1} \mathrm{H}$ NMR spectrum (500 MHz, $\mathrm{CDCl}_{3}$ )

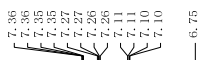
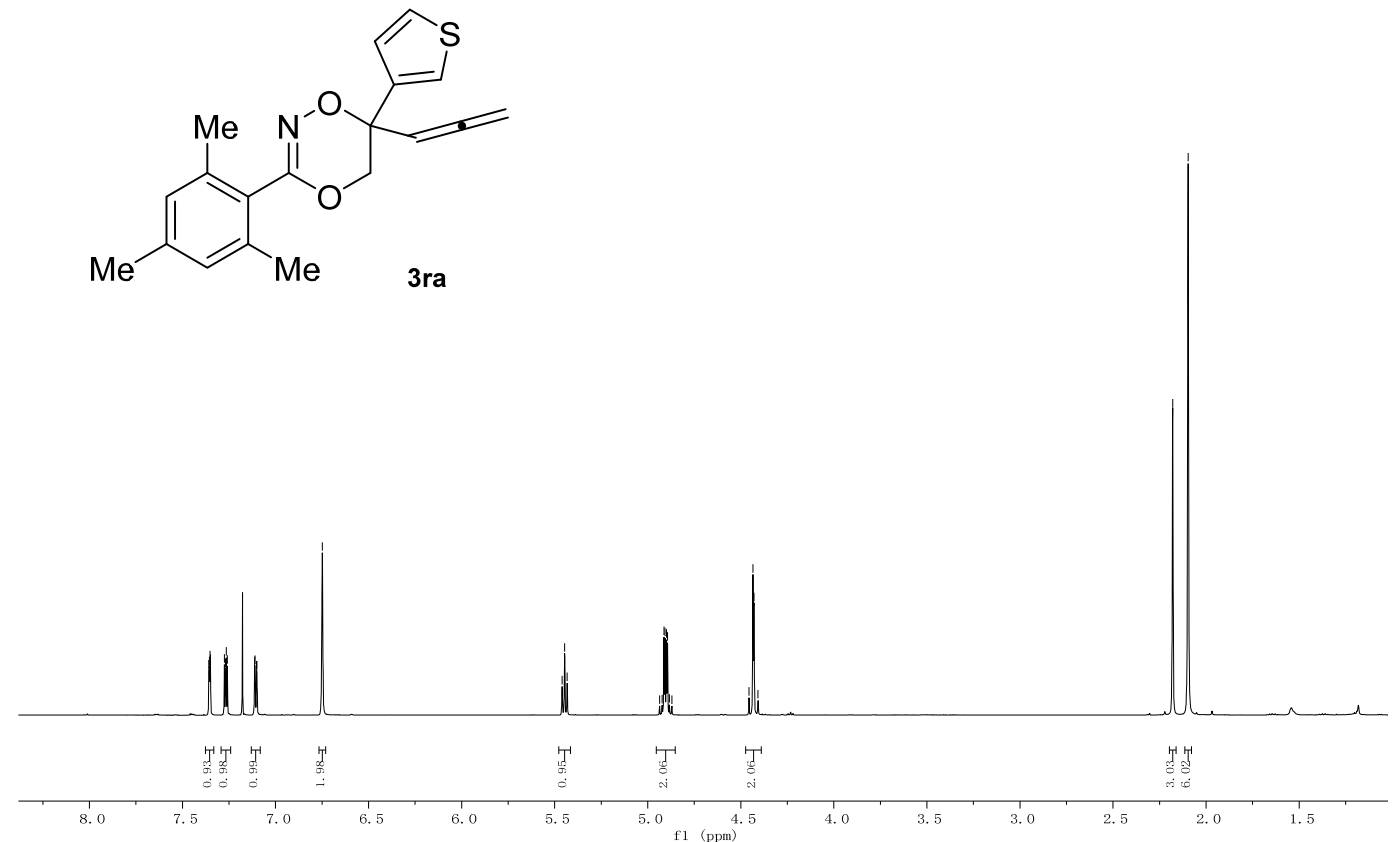

${ }^{13} \mathrm{C}$ NMR spectrum $\left(126 \mathrm{MHz}, \mathrm{CDCl}_{3}\right)$

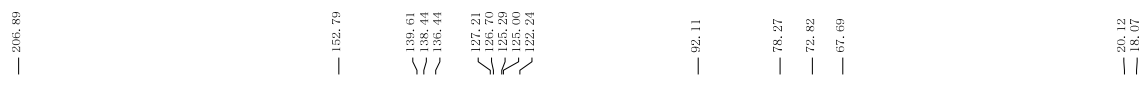

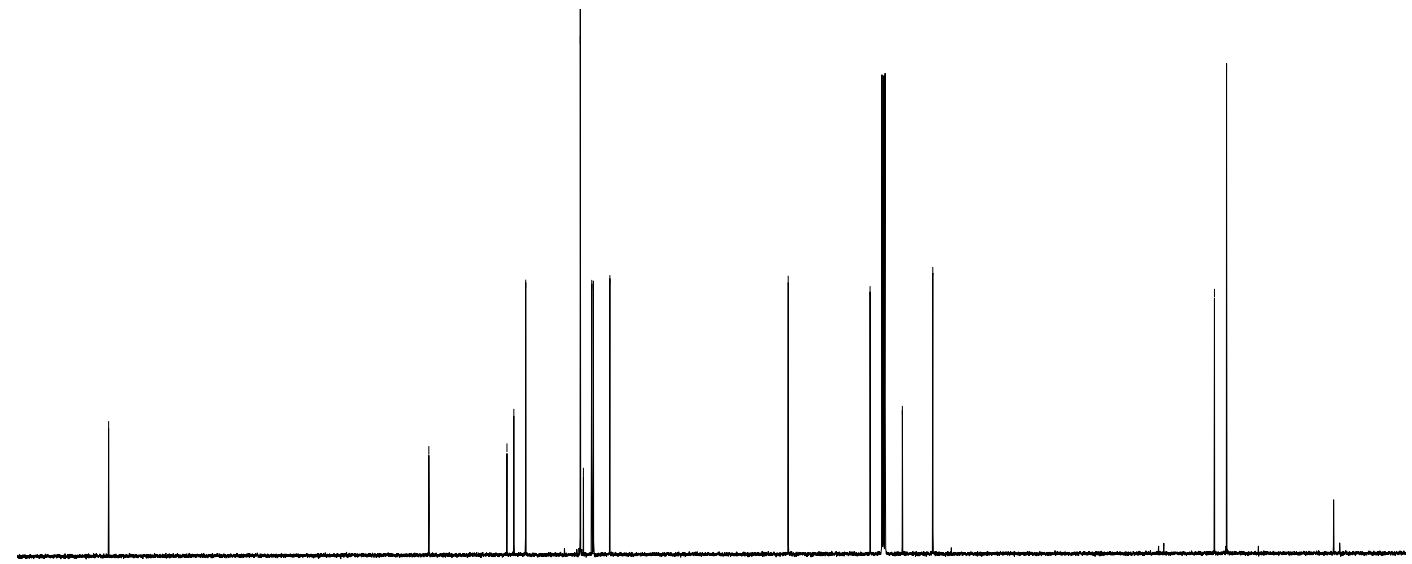

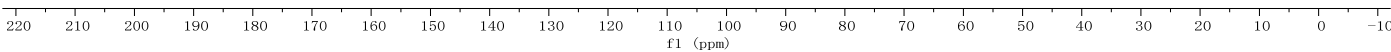


${ }^{1} \mathrm{H}$ NMR spectrum $\left(400 \mathrm{MHz}, \mathrm{CDCl}_{3}\right)$

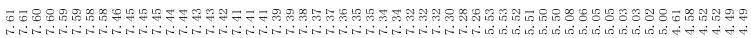<smiles>C=CC1(c2ccccc2)COC(c2c(Cl)cccc2Cl)=NO1</smiles>

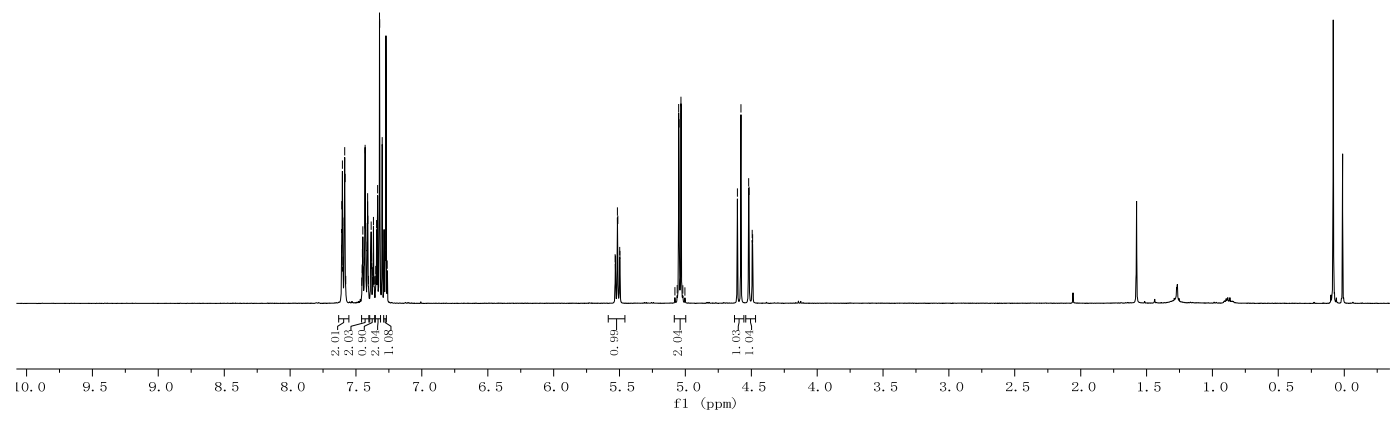

${ }^{13} \mathrm{C}$ NMR spectrum $\left(126 \mathrm{MHz}, \mathrm{CDCl}_{3}\right)$

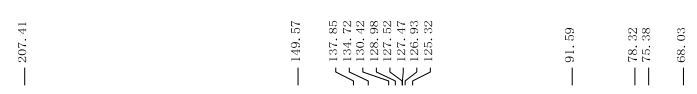
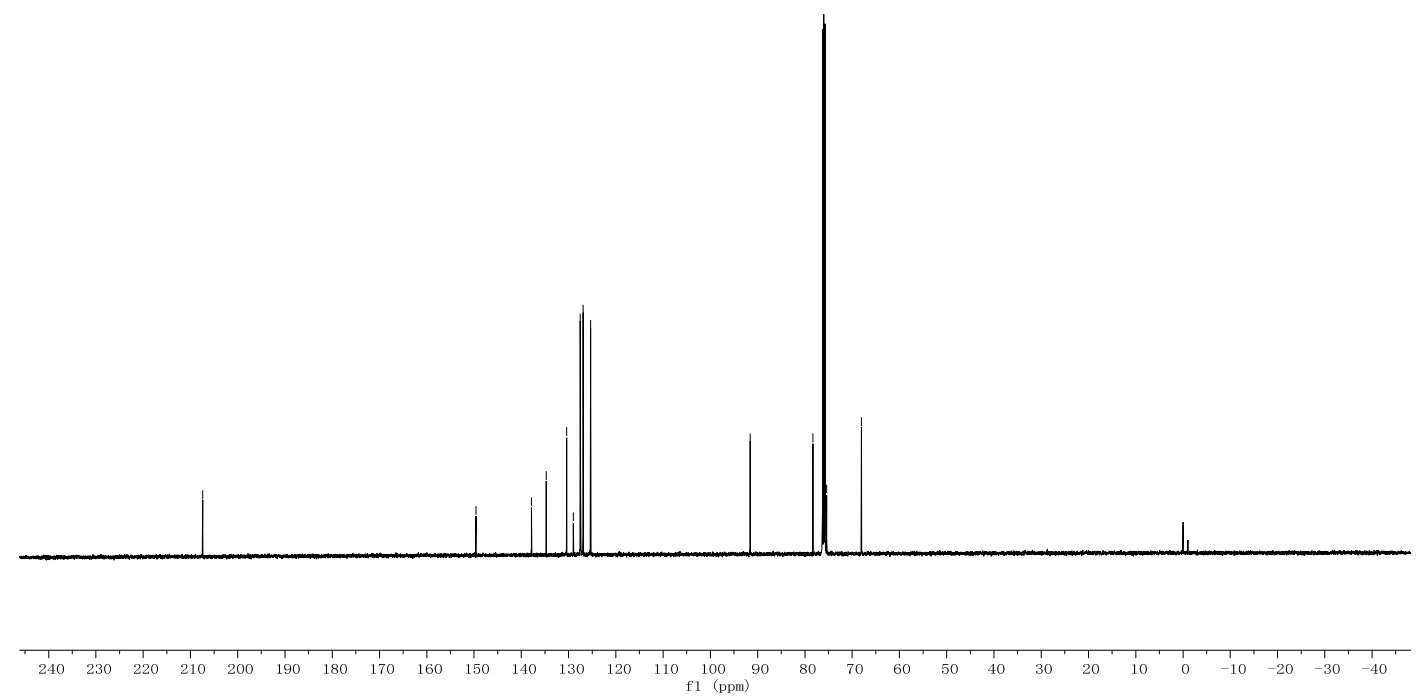
${ }^{1} \mathrm{H}$ NMR spectrum $\left(400 \mathrm{MHz}, \mathrm{CDCl}_{3}\right)$
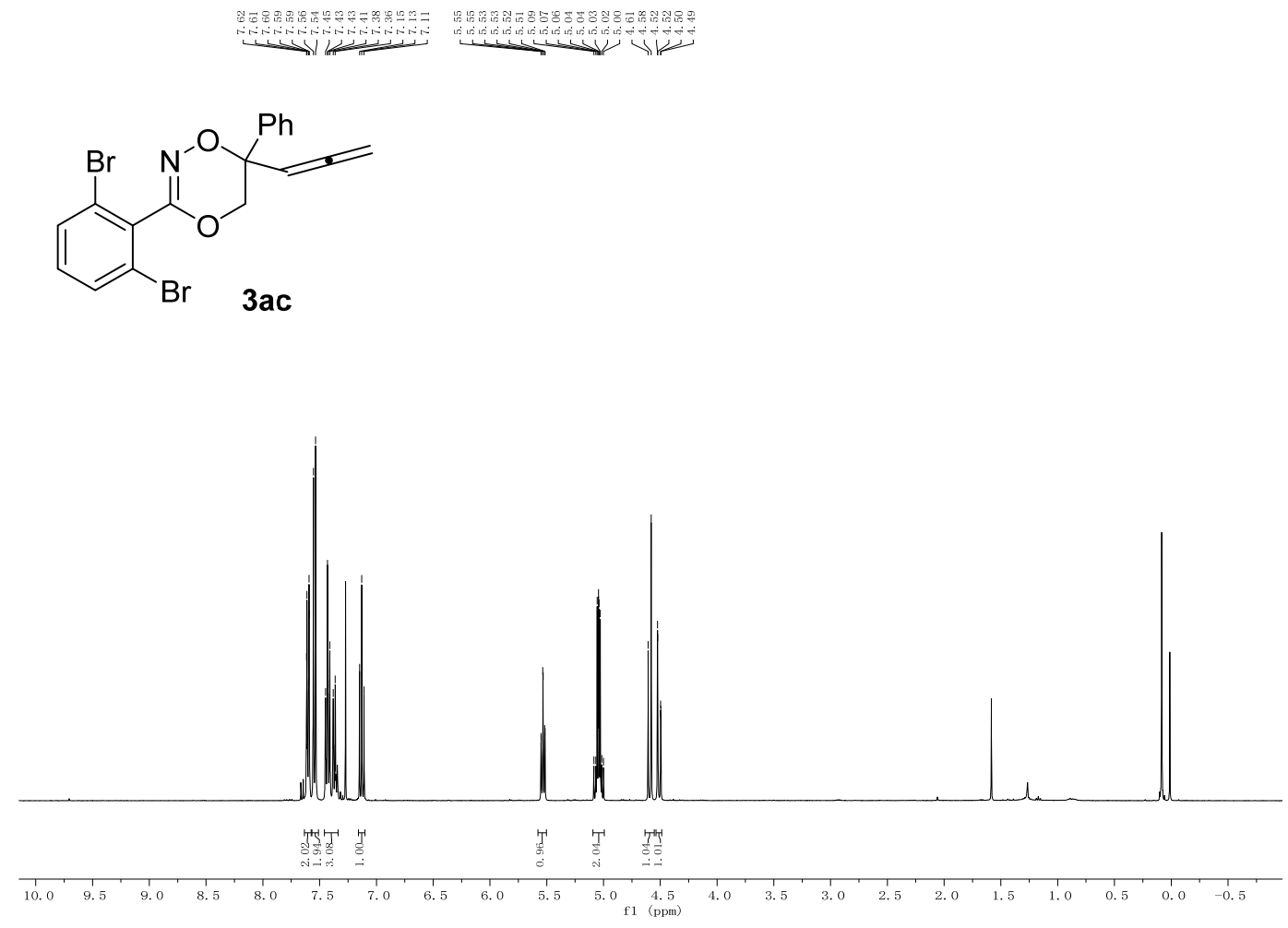

${ }^{13} \mathrm{C}$ NMR spectrum $\left(126 \mathrm{MHz}, \mathrm{CDCl}_{3}\right)$
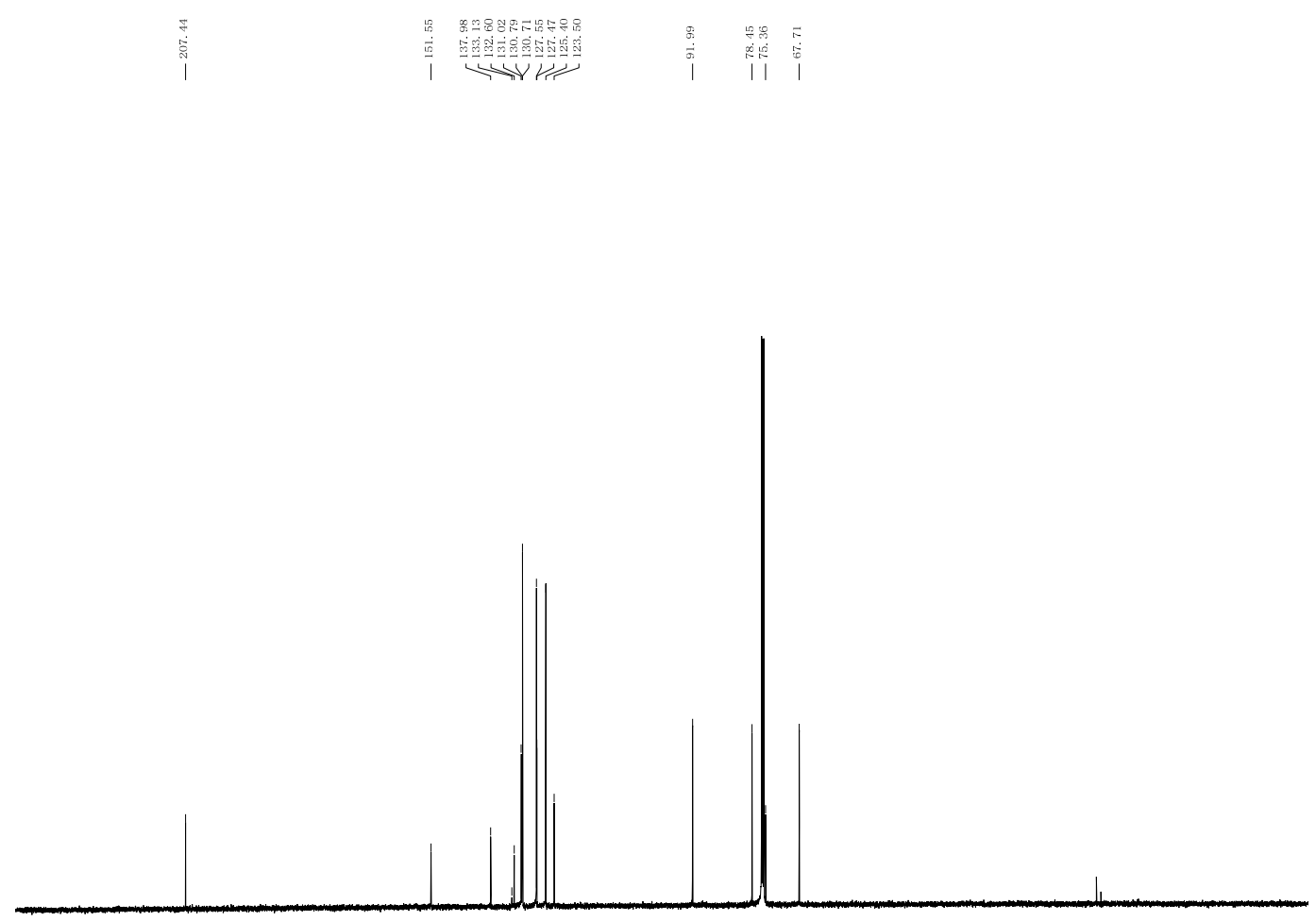

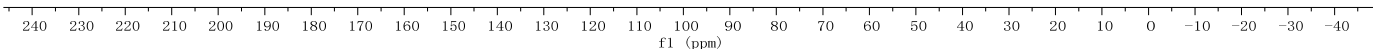


${ }^{1} \mathrm{H}$ NMR spectrum $\left(500 \mathrm{MHz}, \mathrm{CDCl}_{3}\right)$

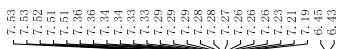
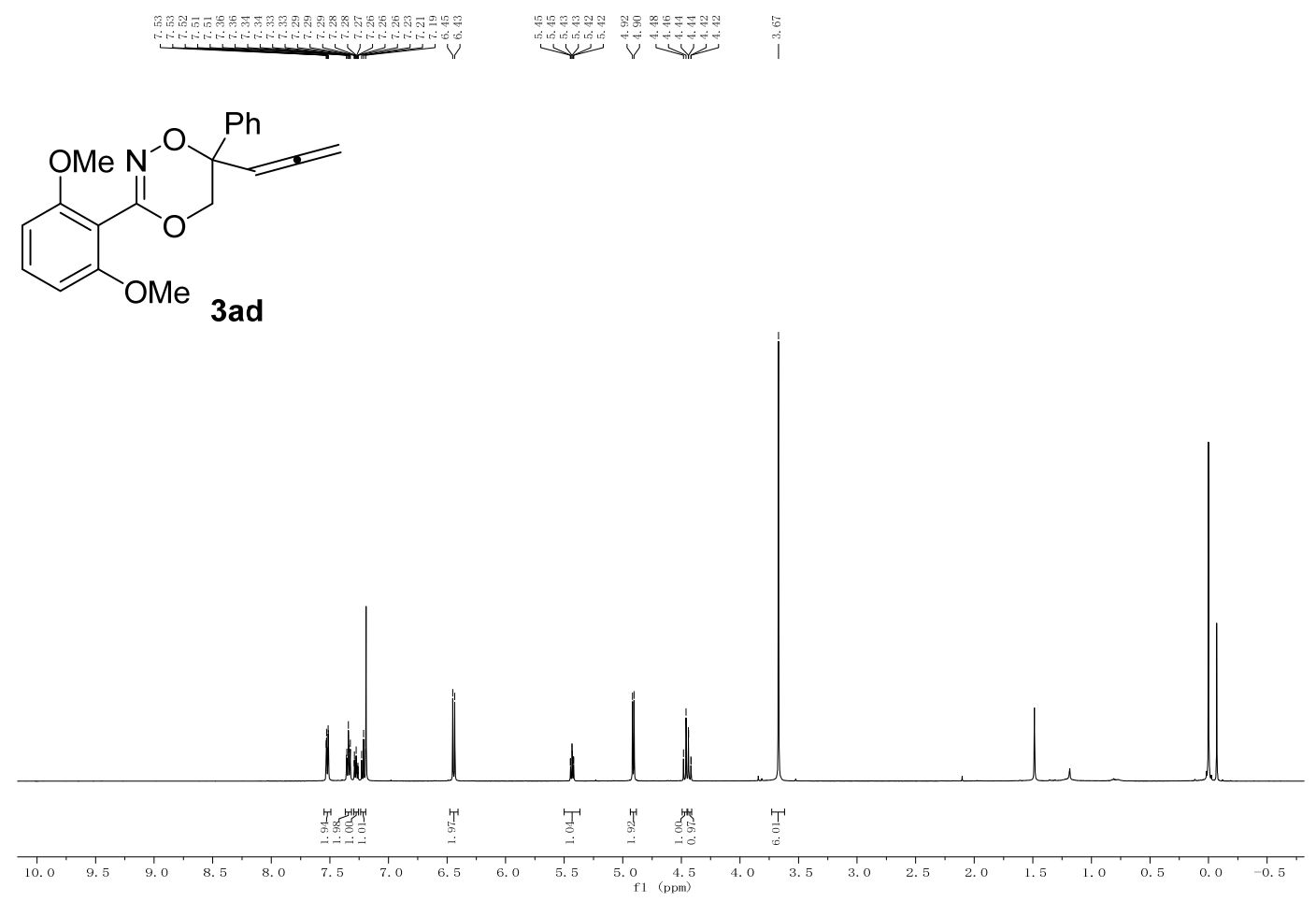

${ }^{13} \mathrm{C}$ NMR spectrum $\left(126 \mathrm{MHz}, \mathrm{CDCl}_{3}\right)$
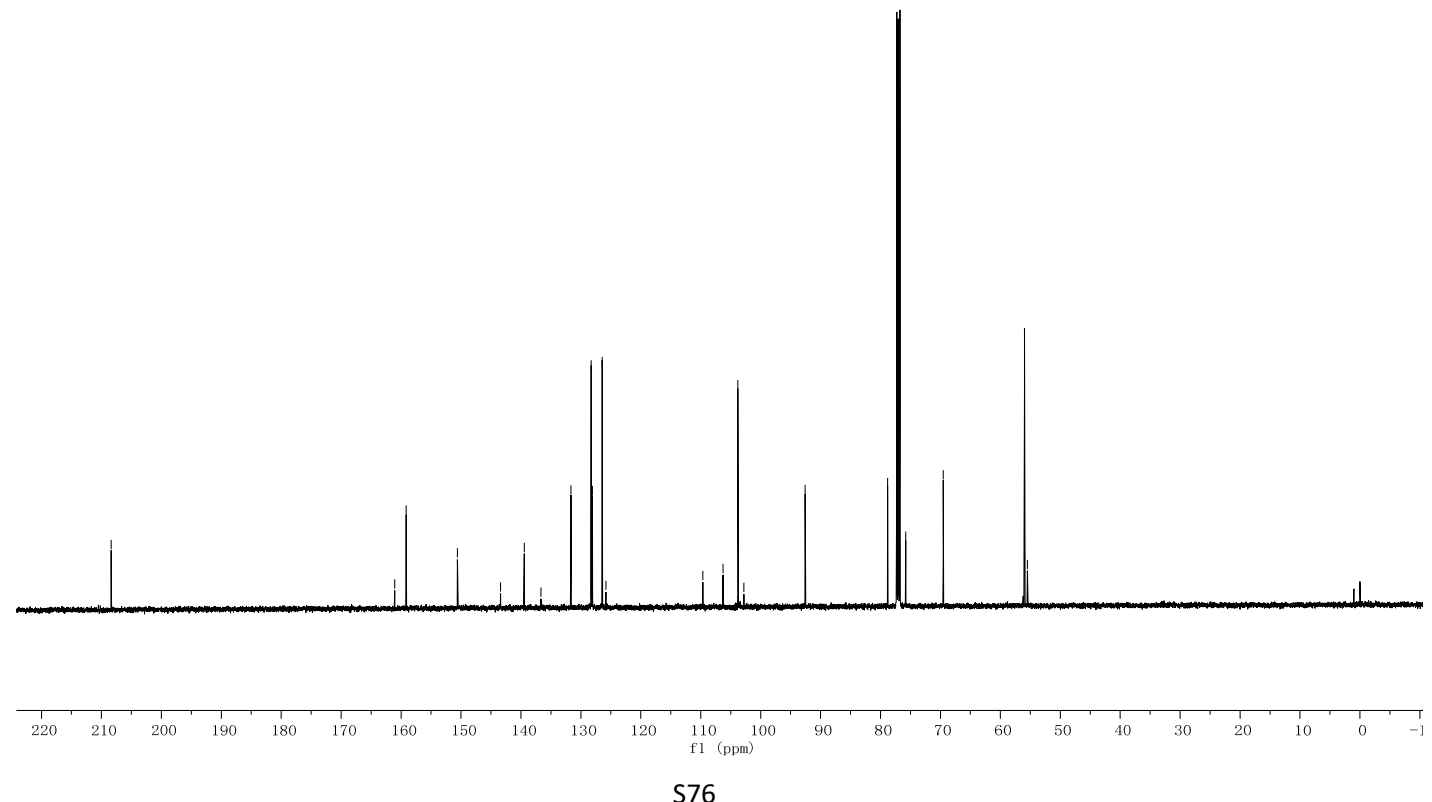
${ }^{1} \mathrm{H}$ NMR spectrum (400 MHz, $\mathrm{CDCl}_{3}$ )

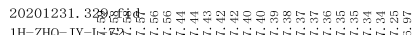

$1 \mathrm{H}-\mathrm{ZHQ}-\mathrm{JY}-\mathrm{TH}$

$\underbrace{2}$
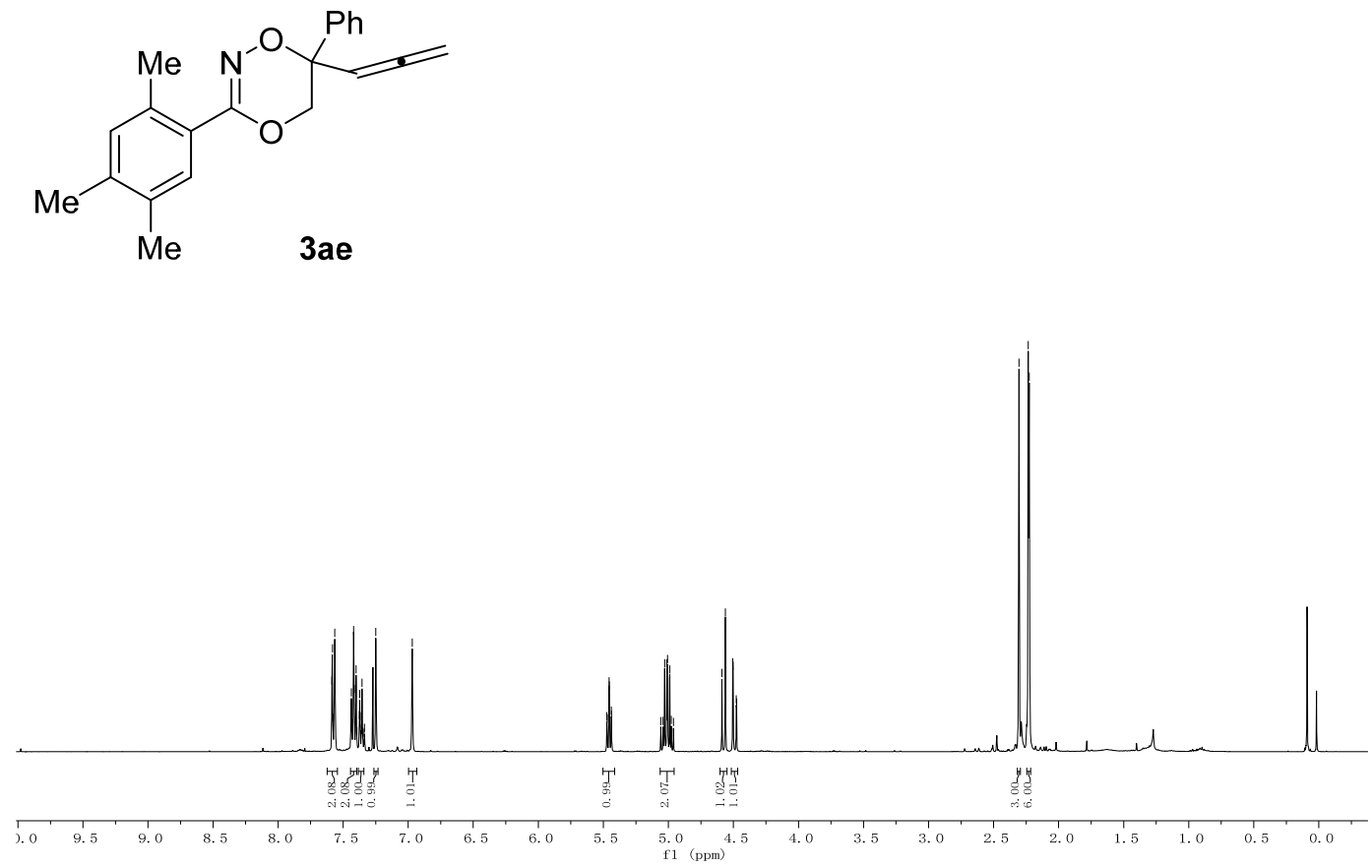

${ }^{13} \mathrm{C}$ NMR spectrum $\left(126 \mathrm{MHz}, \mathrm{CDCl}_{3}\right)$

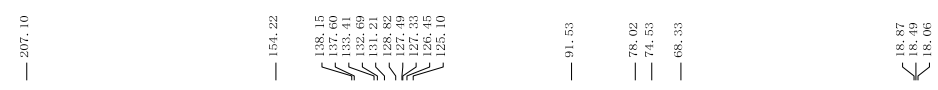

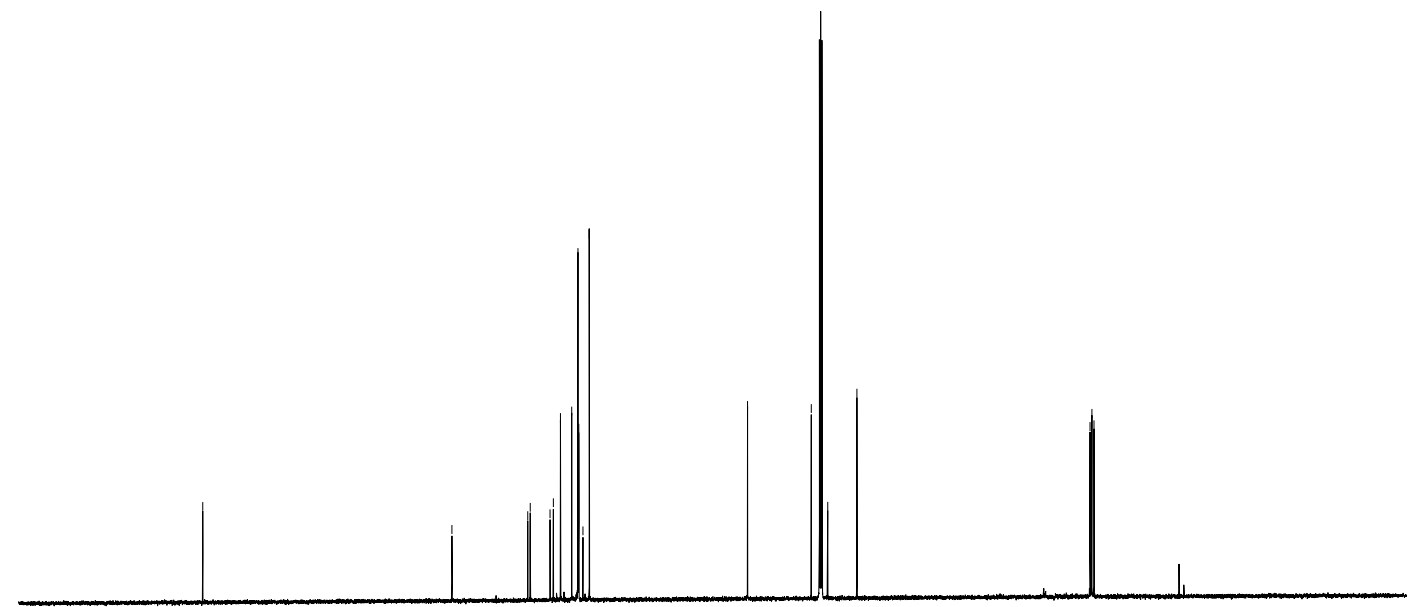

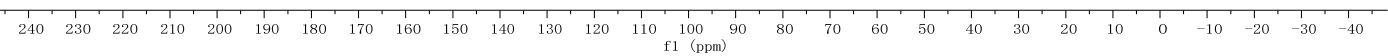
S77 
${ }^{1} \mathrm{H}$ NMR spectrum $\left(400 \mathrm{MHz}, \mathrm{CDCl}_{3}\right)$

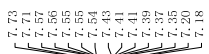

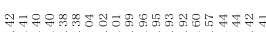

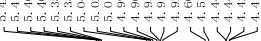
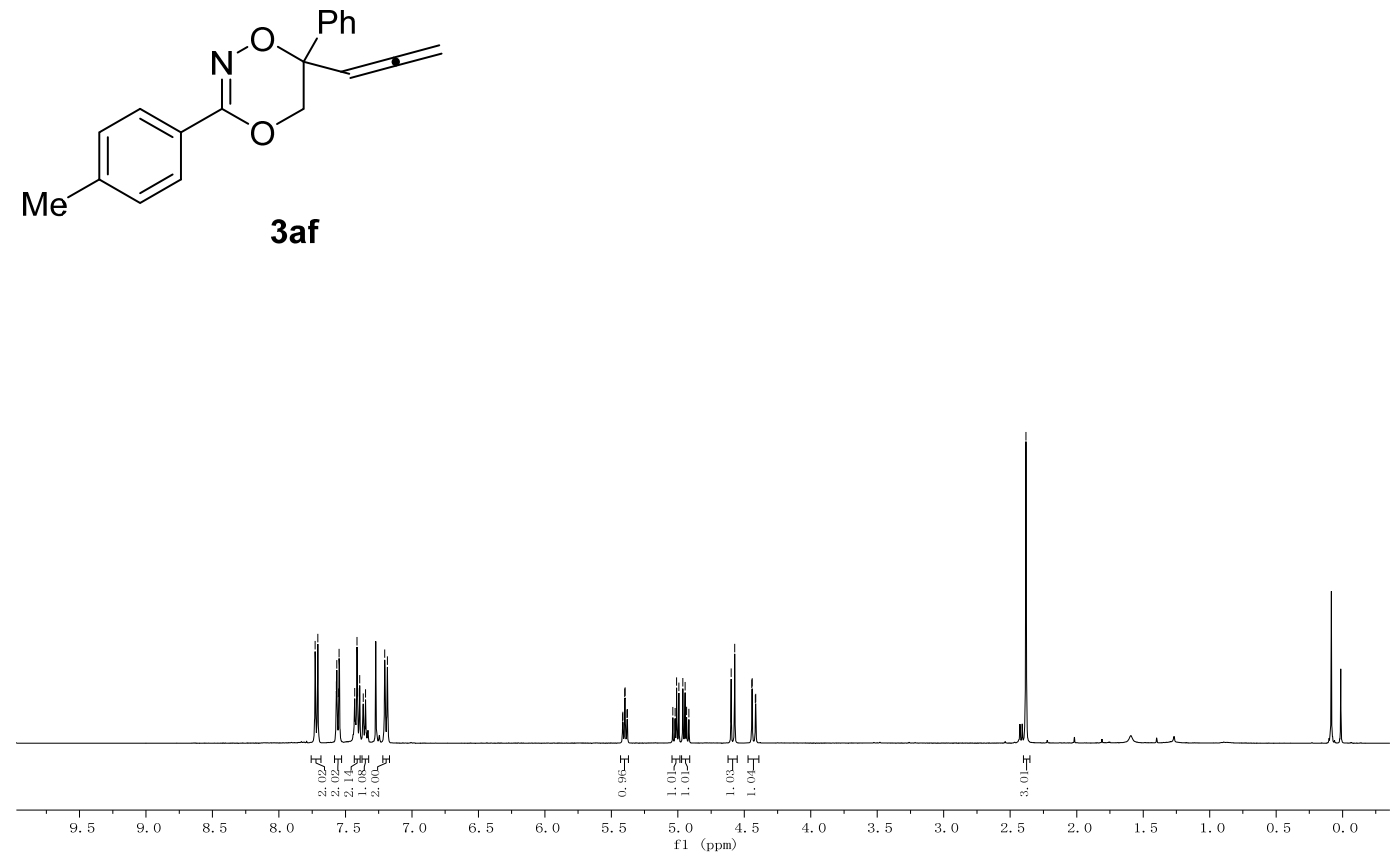

${ }^{13} \mathrm{C}$ NMR spectrum $\left(126 \mathrm{MHz}, \mathrm{CDCl}_{3}\right)$
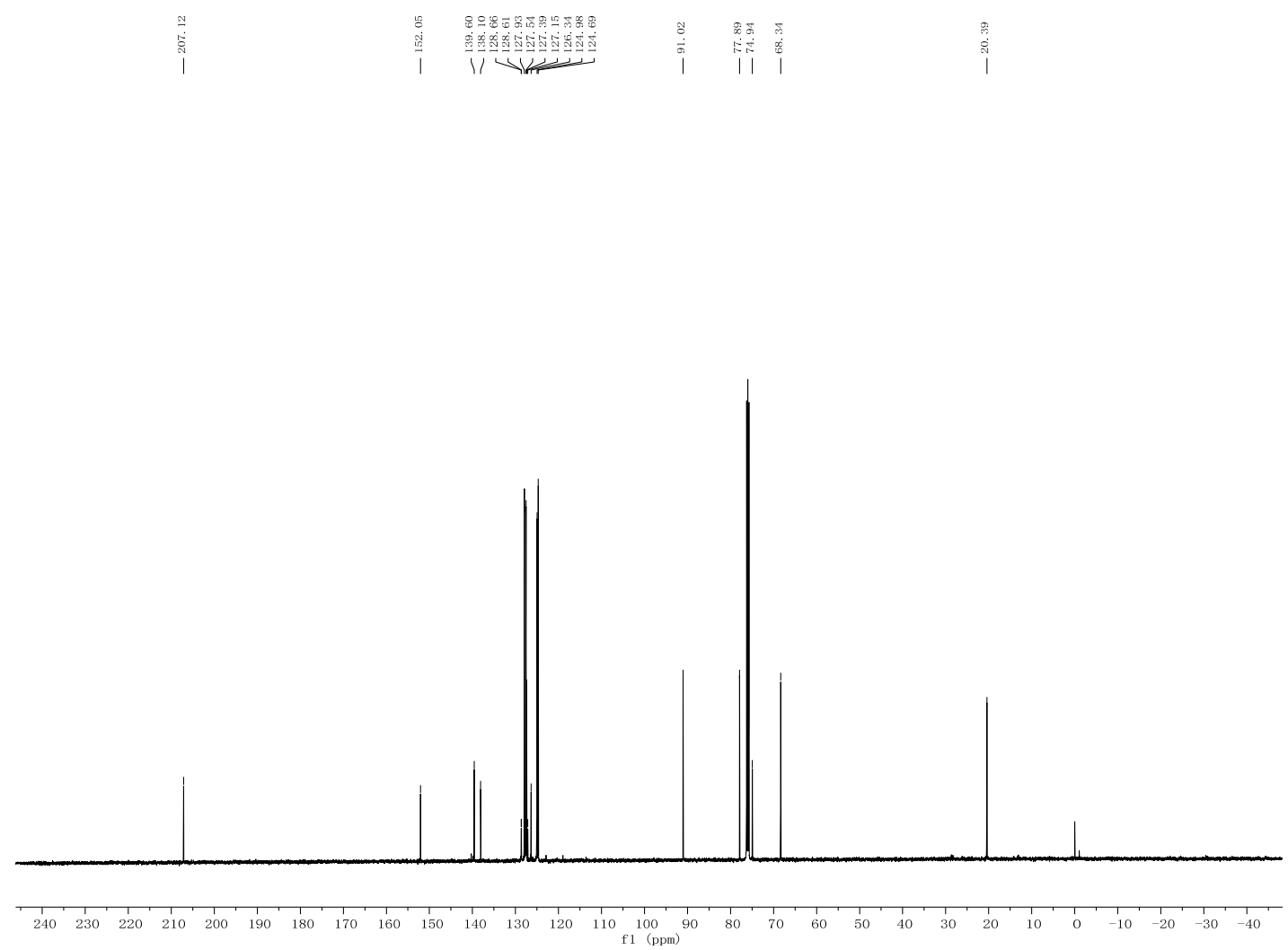
${ }^{1} \mathrm{H}$ NMR spectrum (500 MHz, $\mathrm{CDCl}_{3}$ )
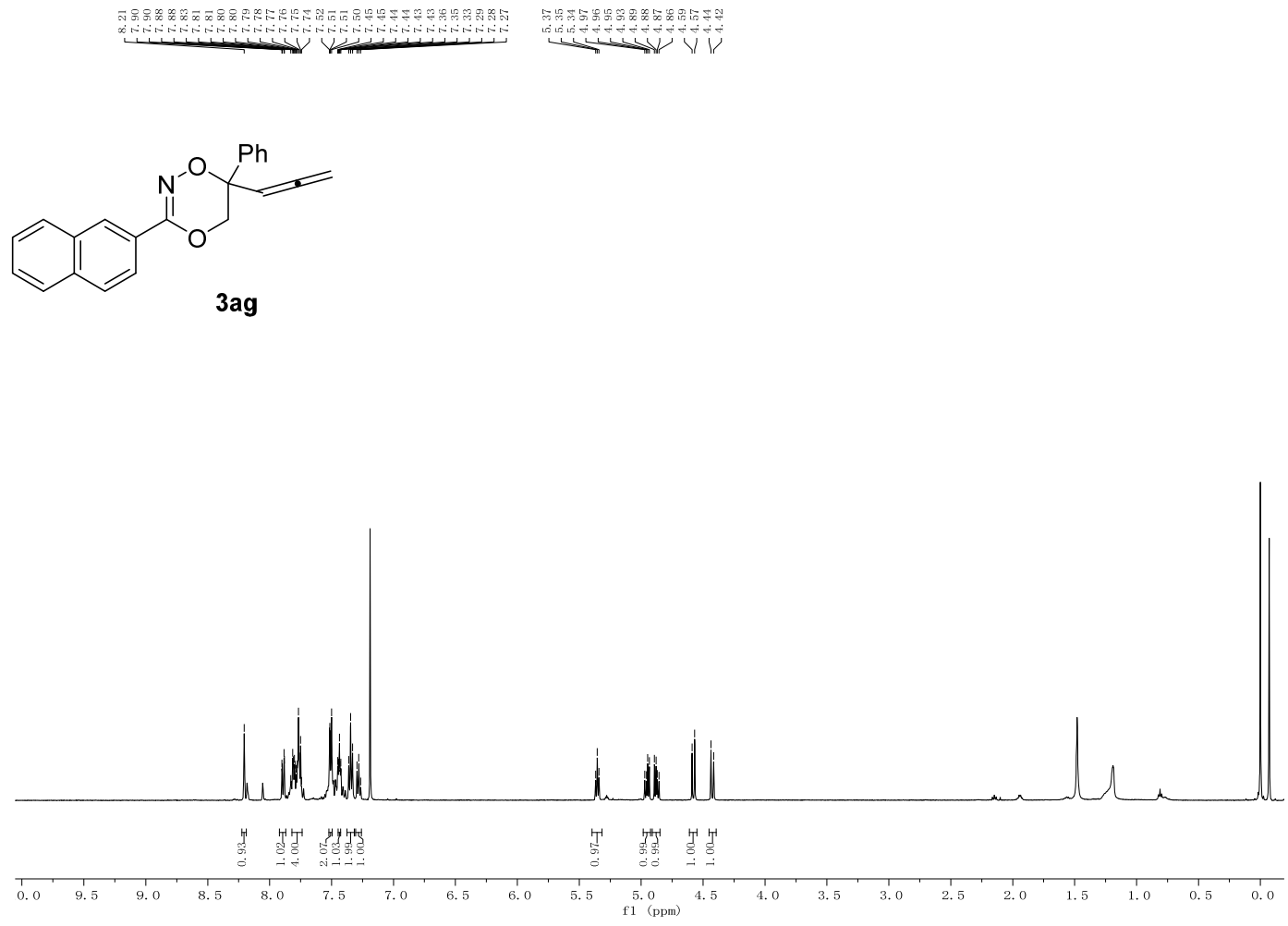

${ }^{13} \mathrm{C}$ NMR spectrum $\left(126 \mathrm{MHz}, \mathrm{CDCl}_{3}\right)$

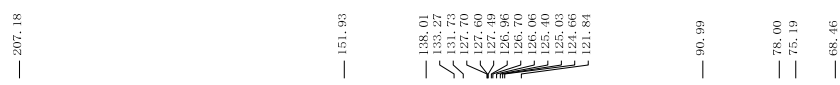
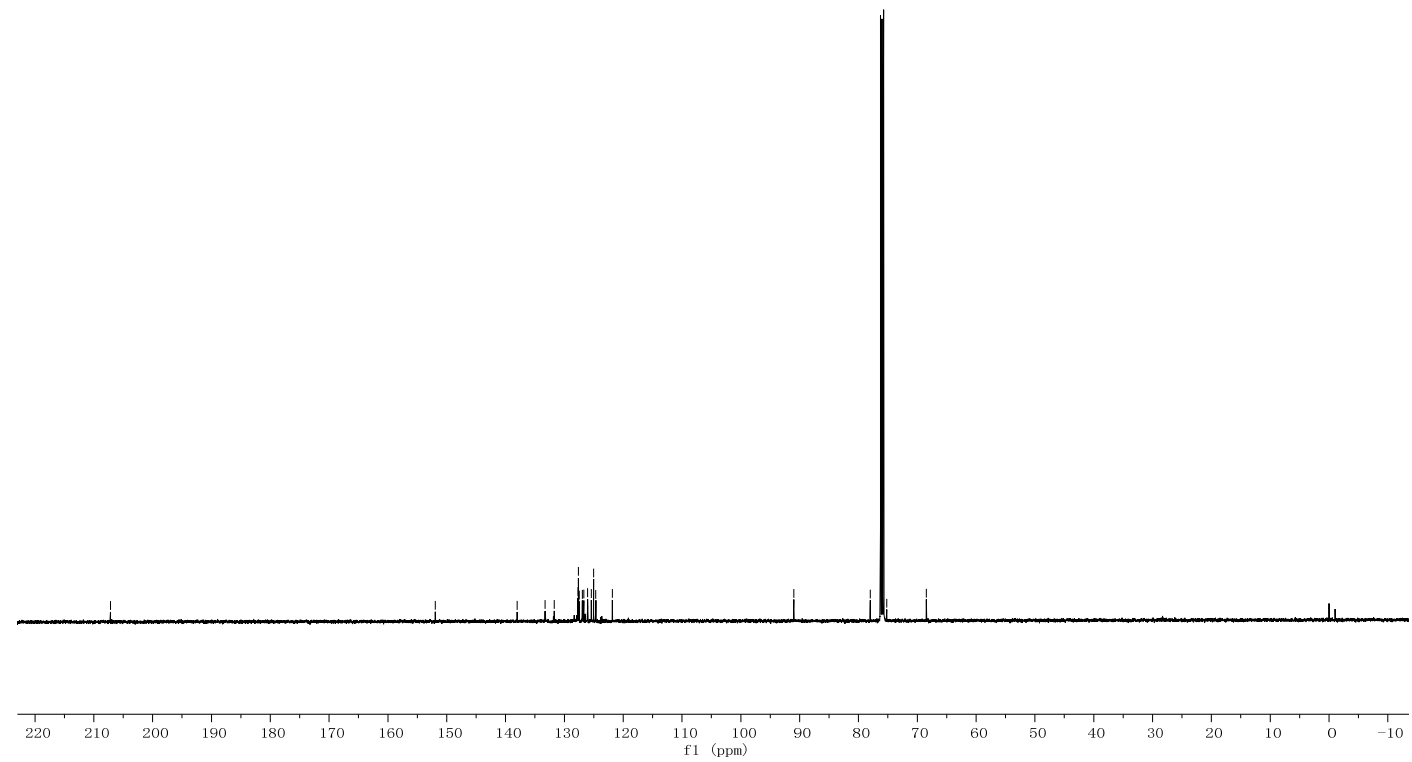
${ }^{1} \mathrm{H}$ NMR spectrum $\left(500 \mathrm{MHz}, \mathrm{CDCl}_{3}\right)$
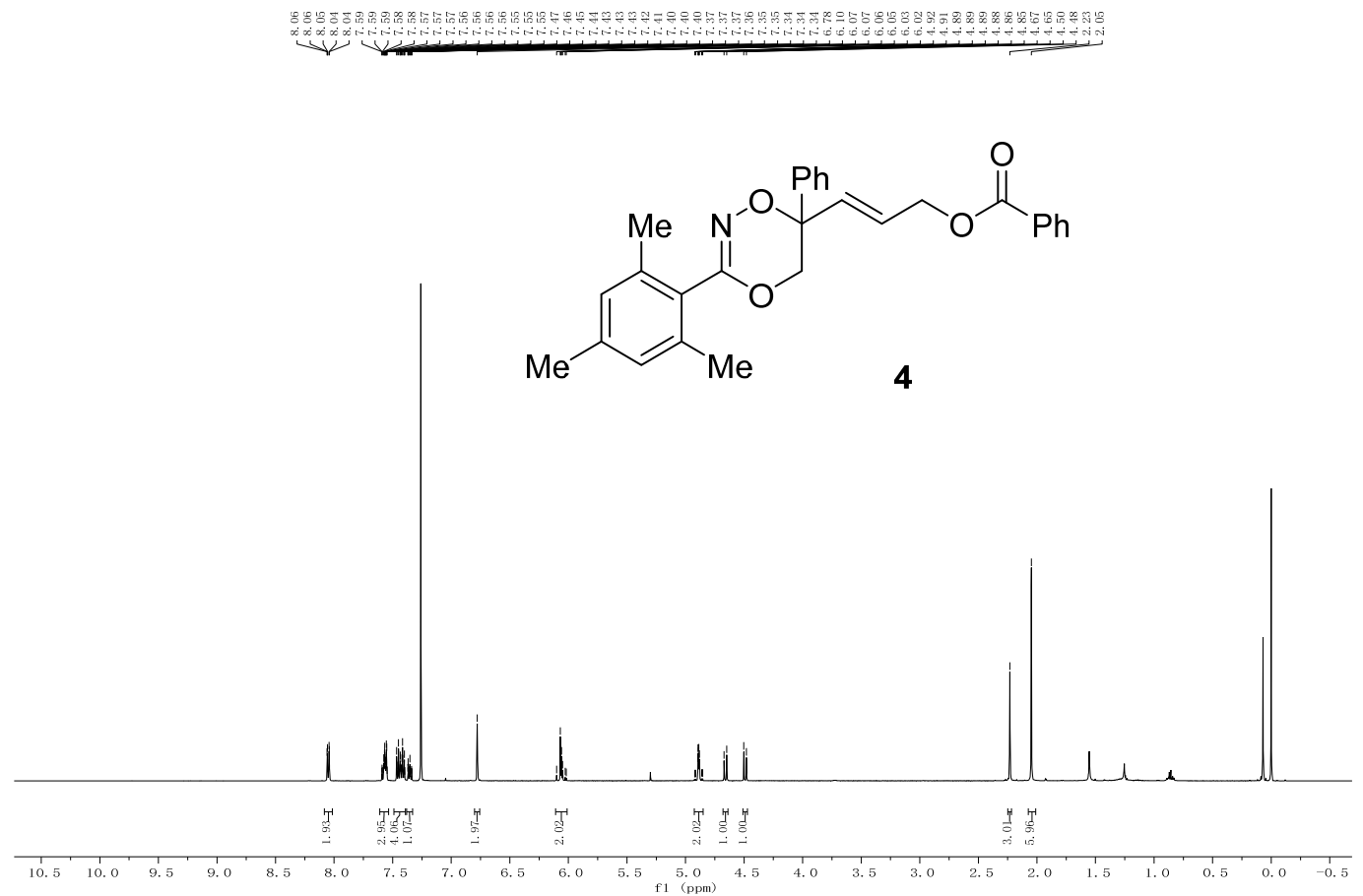

${ }^{13} \mathrm{C}$ NMR spectrum $\left(126 \mathrm{MHz}, \mathrm{CDCl}_{3}\right)$

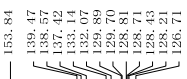

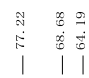

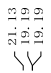

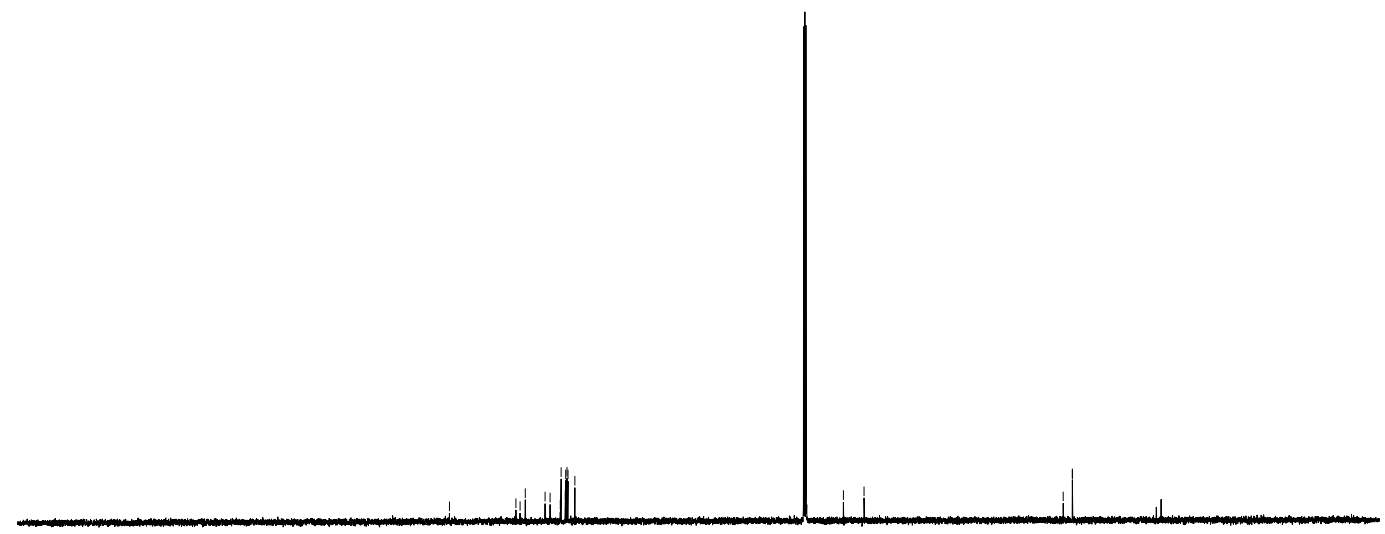

$\begin{array}{lllllllllllllllllllllllllllllllllllllllll}240 & 230 & 220 & 210 & 200 & 190 & 180 & 170 & 160 & 150 & 140 & 130 & 120 & 110 & 100 & 90 & 80 & 70 & 60 & 50 & 40 & 30 & 20 & 10 & 0 & -10 & -20 & -30 & -40\end{array}$ 
HPLC chromatogram of racemic product $3 a a$

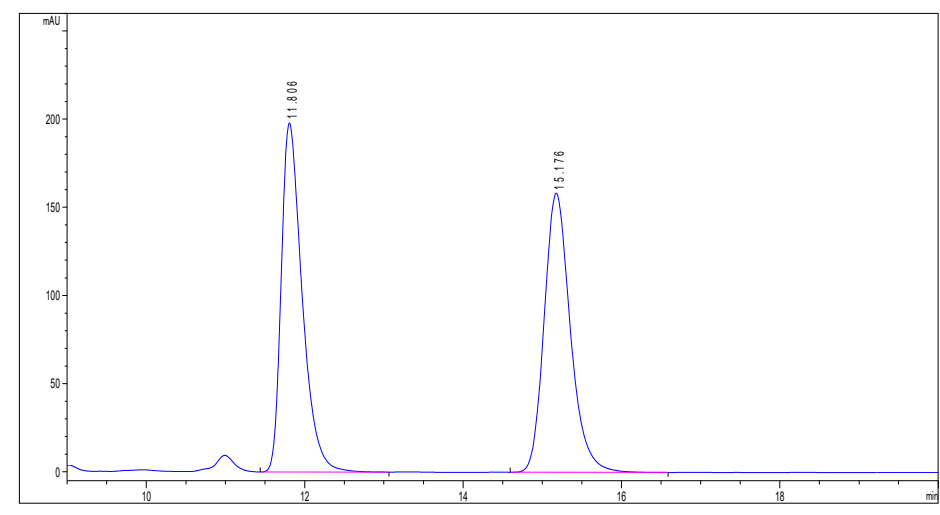

\begin{tabular}{ccccccc}
\multicolumn{2}{c}{ Peak RetTime Type } & Width & Area & Height & \multicolumn{2}{c}{ Area } \\
$\#$ & {$[\mathrm{~min}]$} & {$[\mathrm{min}]$} & $\mathrm{mAU}$ & $* \mathrm{~s}$ & {$[\mathrm{mAU}]$} & $\%$ \\
$-----|----------|$ & & & & & & \\
1 & 11.806 & BB & 0.2775 & 3622.31421 & 197.92798 & 49.9479 \\
2 & 15.176 & BB & 0.3506 & 3629.87183 & 158.26865 & 50.0521
\end{tabular}

HPLC chromatogram of chiral product 3aa

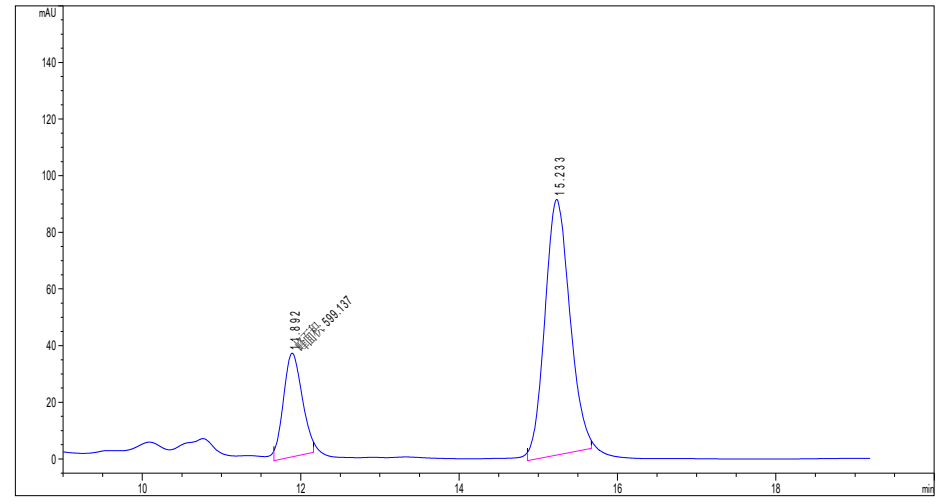

\begin{tabular}{|c|c|c|c|c|c|}
\hline Peak & RetTime Type & Width & Area & Height & Area \\
\hline \# & {$[\mathrm{min}]$} & [min] & $\mathrm{mAU}$ & {$[\mathrm{mAU}$} & $\%$ \\
\hline 1 & $11.892 \mathrm{MM}$ & 0.2727 & 599.13690 & 36.61263 & 23.2925 \\
\hline 2 & $15.233 \mathrm{MM}$ & 0.3645 & 1973.08862 & 90.21078 & 76.7075 \\
\hline
\end{tabular}




\section{X-Ray Crystallographic Data}

X-Ray Crystallography Data Crystallographic data for the product 3ab has been deposited with the Cambridge Crystallographic Data Centre as deposition number CCDC 2065630. These data can be obtained free of charge via www.ccdc.cam.ac.uk/data_request/cif, or by emailing data_request@ccdc.cam.ac.uk, or by contacting The Cambridge Crystallographic Data Centre, 12, Union Road, Cambridge CB2 1EZ, UK; fax: +44 1223336033.

Single crystals of the product 3ab were obtained by slow evaporation of a solution containing 3ab in the mixture of petroleum ether and ethyl acetate at room temperature. A suitable crystal was selected and the crystal data and structure refinement results for compound $\mathbf{3} \mathbf{a b}$ are listed in the Table S2.

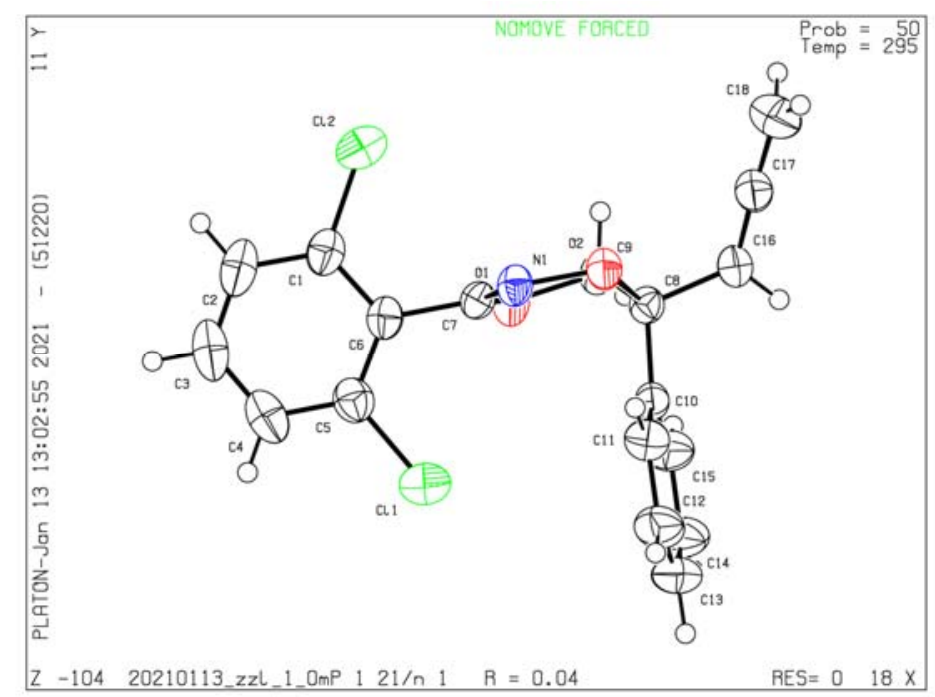

Figure S1. ORTEP view of the compound 3ab with thermal ellipsoids drawn at the $50 \%$ probability level

Table S2. Crystal data and structure refinement for $\mathbf{3 a b}$.

Identification code

Empirical formula

Formula weight

Temperature/K

Wavelength

Crystal system 3ab

$\mathrm{C}_{18} \mathrm{H}_{13} \mathrm{Cl}_{2} \mathrm{NO}_{2}$

346.19

295

$0.71073 \AA$

monoclinic 
Space group

$\mathrm{a} / \AA$

$\mathrm{b} / \AA$

$\mathrm{c} / \AA$

$\alpha /{ }^{\circ}$

$\beta /{ }^{\circ}$

$\gamma /{ }^{\circ}$

Volume $/ \AA^{3}$

Z

Density (calculated)

Absorption coefficient

$\mathrm{F}(000)$

Theta range for data collection

Index ranges

Reflections collected

Independent reflections

Completeness to theta $=25.242^{\circ}$

Max. and min. transmission

Data/restraints/parameters

Goodness-of-fit on $\mathrm{F}^{2}$

Final $\mathrm{R}$ indexes $[\mathrm{I}>2 \operatorname{sigma}(\mathrm{I})]$

Final $\mathrm{R}$ indexes [all data]

Largest diff. peak/hole / e $\AA^{-3}$
P 1211

8.6767(6)

17.6149(12)

$11.3148(7)$

90

107.963(2)

90

$1645.05(19)$

4

$1.398 \mathrm{Mg} / \mathrm{m} 3$

$0.403 \mathrm{~mm}-1$

712.0

2.851 to $27.506^{\circ}$

$-11<=\mathrm{h}<=11,-22<=\mathrm{k}<=22,-14<=1<=14$

23464

$3767\left[\mathrm{R}_{\mathrm{int}}=0.0237\right]$

$99.7 \%$

0.7456 and 0.7157

$3767 / 180 / 216$

1.082

$\mathrm{R}_{1}=0.0427, \mathrm{wR}_{2}=0.1184$

$\mathrm{R}_{1}=0.0506, \mathrm{wR}_{2}=0.1242$

$0.407 /-0.297$ 